\title{
Econophysics and Companies \\ Statistical Life and Death \\ in Complex Business Networks
}

H. Aoyama, Y. Fujiwara, Y. Ikeda,

H. Iyetomi, and W. Souma

Draft copy

August 10, 2009 


\section{Foreword}

This book is one outcome of the new field of econophysics, and explains a wide range of recent findings relating to the dynamics of companies. While economics and physics have a long history of their own, and their methods and purposes are obvious, econophysics, which has only a twenty year track record, is still unfamiliar to many. Indeed, an emerging interdisciplinary approach in which the economy is studied with the tools of physics may provoke doubts as to whether the methods of a hard science can tell us anything about phenomena in which human beings are essential players. However, economics has in fact mimicked physics since the nineteenth century. This is particularly true of those who developed modern economics, the "neoclassical" economists. The old masters such as Alfred Marshall and Léon Walras all drew inspiration from Newtonian mechanics. The fundamental concept of "equilibrium", known to all students of the subject, is, of course, borrowed from physical science.

Thus, a moment's reflection shows us that the relation between physics and economics is long-standing and far closer than is commonly realised. Nevertheless, the recent development of econophysics represents a significant development. While traditional economics learned from classical mechanics, which analyzes behaviours such as that of a ball thrown in the air or the motion of a weight at the end of spring, econophysics looks to the statistical methods of the modern physicist.

Obviously, economic phenomena are comprised of the actions of very large numbers of people and companies. In Japan alone there are over a hundred million people and several million companies, or, in the language of physics, the human population is of order $10^{8}$ and that of companies $10^{6}$. Although these are small numbers in comparison with the everyday quantities of the natural sciences, the Avogadro constant, $\sim 6.02 \times 10^{23}$ for example, it is already impossible to track the movements of all people and companies with any high degree of accuracy. Fortunately for economics, this is not a problem, for while, as individuals, we may be interested in a particular person or a particular firm, economics as a discipline deals with macro phenomena, such as the economy of Japan, or that of Europe as a whole.

In its approach to these macro problems, traditional economics attempts first to analyse the microscopic and then to understand the macroeconomy by a process of scaling up. In other words, standard economics regards the 
macroeconomy as a homothetic enlargement of the representative micro unit. Faced with similar problems in the natural world, statistical physics takes a very different route. Recognising that the micro agents are too numerous to be followed individually, they simply abandon the attempt to capture micro behavior in detail, and employ statistical methods instead. This is the fundamental concept advanced by Maxwell, Boltzmann and Gibbs.

Notwithstanding this precedent, some may still wonder whether it can in principle be meaningful to conduct statistical analysis on social phenomena arising from the actions of individuals, each with an economic motive and a will. Are sophisticated human beings with brains, on the one hand, and inorganic molecules, on the other, really on an equal footing?

More than seventy years ago, when the majority of researchers were opposed to bringing physics into biology, Dr. Torahiko Terada, the major force behind the attempt in Japan, remarked:

When making a statistical analysis of a large number of human individuals we may properly regard it as a mere conglomeration of inorganic material, and altogether neglect individual free will. Indeed, it is now clear that pure physical problems, such as the density of particles in a colloidal matter, may with propriety be compared to statistics of a purely physical nature, such as the "density" or "average speed" of persons walking along street. ... It is sheer folly to dismiss such insights as heresy simply because they are incompatible with the dogma that "living creatures cannot be understood by Physics". Such absurdities remind us that no ignorant amateur poses so serious a threat to progress as a scientist unaware of the nature and goal of their discipline.

Torahiko Terada, "Groups of animals as inorganic groups", Journal of Science, Iwanami Shoten (1933)

The application of physics to biology is now an established discipline, biophysics, and the controversies of the past are quite forgotten. We can confidently expect, not least because of trail-blazing studies such as the current volume, that econophysics will soon seem an equally natural development.

April 2009

Hiroshi Yoshikawa

Professor of Economics, University of Tokyo, Japan 


\section{Preface}

Between their first explorations in econophysics and the writing of this book the authors have travelled a long and sometimes winding road. One of our earliest results was the landmark study of personal income distributions in 2000 (Aoyama et al., 2000), which convinced us that thorough empirical study, or "phenomenology" as it is called in physics, was essential for an understanding of society and economics.

Since then, we have carried out research with an emphasis on the real economy, that is, people (workers), corporations (companies), banks, industrial sectors, and countries. We have also studied the various markets that play a vital role in the activity and prosperity of actual businesses. As a result we began to think of writing a book focused on the real economy and based on the analysis of very large quantities of empirical data. Such work has been largely ignored by economists because that discipline does not, unfortunately, value the empirical search for regularities. Yet, it is this observation-based approach that lies at the root of the success so evident in physics. Kepler's laws of planetary movement, for example, were extracted from the vast quantity of astronomical data collected by Tycho Brahe and others. There is every reason to expect laborious but ingenious analysis of economic data to lead to progress, perhaps not as dramatic as that of Kepler, but progress nonetheless.

We hope that this book will serve as a source-book for people like ourselves who want to move the field of econophysics over to the study of practical economics and companies, rather than the current focus on the application of statistical physics to financial risk.

We shall let our three Tuscans discuss the whole subject in Prologue and Epilogue, after our sincere acknowledgements: Needless to say, many people assisted in research behind this book. High-accuracy, high-frequency data are a must for detailed study of various economic agents, and we would like to thank the Credit Risk Database (CRD) association and its chairman Shigeru Hikuma for general help and advice on the nature of their database, the Organization for Small and Medium Enterprises and Regional Innovation (SMRJ) for help in relation to bankruptcy data, and Tokyo Shoko Research, Ltd. for assistance relating to chain-bankruptcy.

Many other collaborators have contributed to this book in direct and indirect ways at various stages of our research. Our thanks to all, particularly to the following: Masanao Aoki (Los Angeles), Mauro Gallegati (Ancona), 
Corrado Di Guilmi (Ancona), Yasuyuki Kuratsu (Tokyo), Hiroyasu Inoue (Osaka), Taisei Kaizoji (Tokyo), Makoto Nirei (Tokyo), Hideaki Takayasu (Tokyo), Misako Takayasu (Tokyo), Schumpeter Tamada (Hyogo), and Hiroshi Yoshikawa (Tokyo).

We are also grateful to the Yukawa Institute for Theoretical Physics at Kyoto University for allowing us to use the computing facility for part of our numerical computation.

Thanks also to Nao-san for the illustrations, and to John Constable who has not only read the text in its entirety and brushed up and polished the English of our text, but also made many helpful comments.

Finally, we wish to thank Hitachi, Ltd. and Hitachi Research Institute, which have provided us with research funding for this project. The authors of a work on economics are perhaps more aware than most of just how important such support can be to labourers in the intellectual vineyard. 


\section{Contents}

Foreword

Preface $v$

Prologue xi

1 New Insights 1

1.1 A Scientific Approach . . . . . . . . . . . . . . . . 1

1.1.1 Science of Complex Systems . . . . . . . . . . . . . 3

1.1.2 The Emergence of Econophysics _. . . . . . . . . 3

1.2 Distributions and Fluctuations $\ldots \ldots \ldots \ldots \ldots$

1.3 Are Networks Complex? . . . . . . . . . . . . . . . . . 7

1.4 Change of Environment Surrounding Companies . . . . . . . 8

1.4.1 Outline of the Japanese Electric Machinery and Automobile Industries . . . . . . . . . . . . . . . . . . 8

1.4.2 The Electric Machinery Industry . . . . . . . . . . . . . 9

1.4 .3 Automobile Industry . . . . . . . . . . . . . . . . . 11

1.4.4 Industrial Structures and Business Network . . . . . . 12

2 Size Distribution 15

2.1 Preliminaries . . . . . . . . . . . . . . . . . . . 15

2.1.1 Flows and Stocks . . . . . . . . . . . . . 15

2.1.2 Size Distribution and Pareto's Law . . . . . . . . . 16

2.1.3 Other Distributions with a Long Tail . . . . . . . . . . 23

2.2 Distribution of Personal Income . . . . . . . . . . . . . . . 23

2.2.1 Income Distribution and Pareto's Law . . . . . . . . . 25

2.3 Distribution of Companies . . . . . . . . . . . . . . . . . 29

2.3.1 Size Distribution of Companies . . . . . . . . . . . . 29

2.3.2 Size of European Companies . . . . . . . . . . . . 32

2.3.3 A Caveat: Sample and True Distributions . . . . . . . . 33

2.4 Pareto's law . . . . . . . . . . . . . . . . . . . . 35

2.4 .1 Gini and Robin Hood . . . . . . . . . . . . . 38

2.4.2 Simulation: the inverse-function method . . . . . . . . 42

2.4.3 Devil's Staircase . . . . . . . . . . . . . . . . . 43 
2.4.4 Oligopoly and Monopoly . . . . . . . . . . . . . 46

2.4.5 Pareto's 80-20 rule . . . . . . . . . . . . . 50

2.4.6 The Fractal Dimension . . . . . . . . . . . . . 55

2.5 Side story: "long-tail phenomena" . . . . . . . . . . . . . 59

$2.6 \mu=1$ and Phase Transition . . . . . . . . . . . . . 61

3 Fluctuations - Company Growth 65

3.1 Gibrat's Law and Detailed Balance . . . . . . . . . . . . . . . 67

3.1.1 Growth Rate and Gibrat's Law . . . . . . . . . . . . 67

3.1.2 Data for Japanese Companies . . . . . . . . . . . . . . 71

3.1.3 Data for European Companies . . . . . . . . . . . 72

3.1.4 Gibrat Revisited . . . . . . . . . . . . . . . 74

3.1 .5 Detailed Balance . . . . . . . . . . . . 76

3.1.6 Relation between Pareto's and Gibrat's Laws and the

Reflection Law . . . . . . . . . . . . . . 80

3.1.7 Copula . . . . . . . . . . . . . . . . . . 82

3.2 Digression: Personal-income Fluctuation . . . . . . . . . . . . 86

3.2.1 Gibrat's Law and Detailed Balance . . . . . . . . . . 86

3.2.2 Breakdown of the Laws . . . . . . . . . . . . . . . . . 89

3.2.3 Side-story: Public Notice of High-Tax Payers, and Lost Data in Japan . . . . . . . . . . . . . . . . . . . . . . 90

3.3 Small and Medium-sized Companies . . . . . . . . . . . . . . 91

3.3.1 Large-scale Data for Small and Medium Enterprises . . 91

3.3.2 Size-Dependence of Growth . . . . . . . . . . . . . . 93

3.4 Companies Bankruptcy . . . . . . . . . . . . . . . . 96

3.4.1 Companies Activity and Bankruptcy . . . . . . . . . . 96

3.4.2 Debt when Bankrupted and Life-time . . . . . . . . . 97

3.5 Production Function and Ridge Theory . . . . . . . . . . . . 100

3.5.1 Production Function . . . . . . . . . . . . . 100

3.5.2 Ridge Theory for Companies Growth . . . . . . . . . . 102

4 Complex Business Networks

111

4.1 Introduction to Network Science . . . . . . . . . . . . . . . . . 112

$4.21,2,3, \ldots, 6$ degrees of separation . . . . . . . . . . . . 118

4.3 Networks in Economy . . . . . . . . . . . . . . . . . . . . . . . 124

4.3.1 The shareholding Network . . . . . . . . . . . . . 125

4.3.2 The Interlocking Directors' Network . . . . . . . . . . 127

4.3.3 The Transaction Network . . . . . . . . . . . . . . . 129

4.3.4 The Innovation Network . . . . . . . . . . . . . . . 131

4.4 Network Indices . . . . . . . . . . . . . . . . . . . . . . . . . . 132

4.4.1 Degree Centrality . . . . . . . . . . . . . . . . . 135

4.4 Shortest Path Length . . . . . . . . . . . . . . . . 136

4.4 .3 Clustering Coefficient . . . . . . . . . . . 137

4.4.4 The Betweenness Centrality of Nodes . . . . . . . . 138

4.4.5 Cliques . . . . . . . . . . . . . . . . 139

4.5 Statistical Properties of Network Indices . . . . . . . . . . . . 139 
4.5.1 Comparison of Industries by Using Network Indices . . 139

4.5.2 Degree Distribution . . . . . . . . . . . . . . . 142

4.5.3 Correlation related to Degree . . . . . . . . . . . . 145

4.5.4 Shareholding Network and Company Size . . . . . . . . 147

4.6 Dynamics of the Company Network . . . . . . . . . . . . . 149

4.6.1 Change in the Shareholding Network . . . . . . . . . 150

4.6.2 Change of Degree Distribution . . . . . . . . . . . 153

4.6.3 Correlation between Companies in Networks . . . . . 158

5 Agent-Based Model for Companies 169

5.1 Gibrat's Process . . . . . . . . . . . . . . . . . 169

5.2 Model of Shareholding Network . . . . . . . . . . . . . . . . 172

5.2.1 Reproduction of Size Distribution . . . . . . . . . . . 172

5.2.2 Reproduction of Degree Distribution . . . . . . . . . . . 174

5.2.3 Effects of Nodal Characteristics . . . . . . . . . . . 175

5.3 Balance Sheet Dynamics . . . . . . . . . . . . . . . 176

5.3.1 The Basic Agent Model . . . . . . . . . . . . . . . 177

5.3 .2 Representative Agents . . . . . . . . . . . . . . . 181

5.3 .3 Reduction to a Multiplicative Process . . . . . . . . . . 181

5.3.4 Distribution of Company Sizes . . . . . . . . . . . . . 182

5.3.5 Synchronised Bankruptcy . . . . . . . . . . . . . . 185

5.4 Network Effects on Wealth Distribution . . . . . . . . . . . . . 188

5.4 .1 Model Construction . . . . . . . . . . . . . . 188

5.4 .2 Network Effects . . . . . . . . . . . . . . . . . . . 189

5.4 .3 Clustering of Wealth . . . . . . . . . . . . . . 190

5.5 Modelling the Transaction Network . . . . . . . . . . . . . . 193

5.5.1 Autonomous Companies . . . . . . . . . . . . . . . 194

5.5.2 Model of Bounded Rationality . . . . . . . . . . . . 199

6 Perspectives for Practical Applications 205

6.1 Development of Business Strategies . . . . . . . . . . . . . 205

6.1.1 Valuation of Companies . . . . . . . . . . 206

6.1.2 Optimum Capital Structure . . . . . . . . . . . . . 209

6.1.3 Decision-Making for Business Entry and Exit . . . . . . 211

6.1.4 Decision-making under a Given Economic Trend . . . . 214

6.2 Chain Bankruptcy and Credit Risk . . . . . . . . . . . . . 217

6.2.1 Transaction Network . . . . . . . . . . . . . 217

6.2.2 The Relationship of Debtors and Creditors . . . . . . 219

6.2.3 The Causes of Bankruptcy and the Link Effect . . . . . 220

6.2.4 Magnitude of Link Effect . . . . . . . . . . . . . . . 221

6.2.5 The Ripple Effect . . . . . . . . . . . . . . . . . 222

6.2.6 Propagation of Credit Risk on the Transaction Network 230

6.3 Business Model and Business Information . . . . . . . . . . . 232

6.3.1 The Industrial Group as a Business Model . . . . . . . . . 234

6.3 .2 Robustness of Industrial Group . . . . . . . . . . . . . . 238

6.3 .3 Synergy in Industrial Groups . . . . . . . . . . . . . 239 
6.3.4 Business Information Systems . . . . . . . . . . . 241

$\begin{array}{ll}\text { Epilogue } & 247\end{array}$

$\begin{array}{ll}\text { List of Figures } & 251\end{array}$

$\begin{array}{ll}\text { List of Tables } & 257\end{array}$

$\begin{array}{ll}\text { Bibliography } & 259\end{array}$

$\begin{array}{ll}\text { Index } & 268\end{array}$

$\begin{array}{ll}\text { About the authors } & \mathbf{2 7 6}\end{array}$ 


\section{Prologue}

I have for many years been a partisan of the Copernican view because it reveals to me the causes of many natural phenomena that are entirely incomprehensible in the light of the generally accepted hypothesis.

- Galileo Galilei in a letter to Johannes Kepler

SAlv.: Greetings, Sagredo, Simplicio, my good friends. I can hardly believe that it was only yesterday that we resolved to meet and talk about this book. How the time drags when I am not in pleasant company such as yours.

Simp.: Greetings to you, most courteous Salviati, and well met, well met I say. My mind is already racing in anticipation. I have not forgotten, and could not forget, our wonderful discussions with Professor Galileo in Tuscany, and I am convinced that on this occasion too you have found something worth the labour of a Dialogue (Galilei, 1632).

SAGR.: For my part I am also delighted to see you both again. In the company of two such philosophers as yourselves I never fail to find inspiration and illumination. Now, would you care to tell me the nature of the subject, Salviati?

SAlv.: Certainly, certainly, shall I come to the point: I feel that a change is happening, just as it was when we met with Professor Galileo.

Simp.: Change! Ha!

SAgr.: Now, now, Simplicio... Let's hear this out. The book is about a change, is it? But I don't understand even the title. What is this Econophysics?

SALV.: You have gone right to the heart of the matter; econophysics is the name of an academic discipline, a name coined in 1995 by that most learned Professor of Boston, Eugene Stanley. He means the word to describe the study of economy or economics as seen through the eyes or analysed with the tools of exact science.

SAGR.: Well that helps me a little, but I am still puzzled by the appearance of the word 'physics' in this new name. Can you explain that, Salviati? 
SALv.: Well that is simple indeed. The main driving force behind this new discipline is the natural science of physics. For, as you will shortly see, statistical physics has many concepts and principles that can be readily applied to phenomena in economics. That is to say, just as economic systems are made of many inhomogeneous agents, like people, companies, and financial institutions, so the natural world studied so successfully by physicists consists of atoms and molecules in gases and condensed matter. The similarity is obvious, is it not?

Simp.: Well so you say, but I need hardly remind you that there is already a long established and well respected discipline studying these matters, namely Economics. You should show a little more respect for that authority, for has not Economics constructed an intellectual context in which economic observations can be placed; namely, the optimization of utility by individual economic agents, the specification of the concept of equilibrium, and the detailed delineation of the implications of the equilibrium model? Who needs this so-called Econophysics?

Salv.: Alas, Simplicio, I fear you have erred in two ways.

SAGR.: Only two? Simplicio's errors are legion and notorious, for example....

Salv.: Piano, piano, Sagredo. Firstly, Simplicio, my dear fellow, undue or unconsidered respect for authority is a prison with invisible walls. Secondly, the context to which you refer is, as it turns out, not entirely suitable for the analysis of economic phenomena. Let me put it in this way, the grounding principle of Econophysics, and this is much more important than the mere import of certain concepts from physical science, is the scientific approach itself, where hypotheses and possible theories are discussed freely in an open manner, tested against determinable facts, and used to make predictions, though not necessarily in that order. And science is really about changing ourselves, our mind, our dearest views, even when we are comfortable and don't wish to be changed, or find alternative views almost impossible to hold in our minds. For example, the quantum theories, or relativistic theories, all of which are beyond our everyday realm, is that not so Simplicio!

Simp.: I am sure you mean no offence, but there is no need to raise your voice, Sagredo, I am listening carefully, though I am not sure yet that I understand your point.

SALV.: Forgive me my dear Simplicio, the subject is of very great importance to me. For you see, the most remarkable thing is that if physicists and economists clear their minds of constraints they can work together in this discipline, and very fruitfully.

SAGR.: So, you say that this book is about the scientific study of companies, companies, firms? 
SAlv.: Yes, yes, the study of the real economy, as it is made up of people, companies, financial institutions, and all this through the lense of exact science. An acquaintance of mine, some sort of poet really, but we need not hold that against him, once said that:

$$
\begin{gathered}
\text { 'Science, .... is the north-west passage } \\
\text { between cynicism and credulity.' }
\end{gathered}
$$

Perhaps it is a little difficult to understand (Poets!), but I think on reflection the matter is clear enough. The challenge before us is to find a way between, one the one hand, a credulous belief in the views of the establishment, whether that is the Church or in this case the academy, on the one hand, and, on the other, a bitter nihilism that tells us to abandon our endeavours because knowledge is impossible and the establishment does no more than reflect the structures of political power prevailing in its time. However, and wonderfully, the methods of science can and do break down the endless circular movement of constrained institutional thought, and, in spite of all the difficulties, these methods also build up a body of facts and understanding on which two or more minds can agree.

SAGR.: Ah yes, I think I can accept this; the method yields understanding without the need to invoke any divine or ultimate foundations to knowledge.

SALV.: Precisely, but as yet in Economics there is a lack of progress academically..

Simp.: Well! I'm not sure everyone would agree with that.

Salv.: Perhaps not, but you have to admit that Economics is not a powerfully predictive or technological science yet.

SAGr.: That would be difficult to dispute, sadly.

SALV.: But we need not despair, this book argues that there is a way through.

SAGR.: That is most encouraging. I'll make a start straight away; I hope it isn't too difficult.

SAlv.: Certainly not. The authors told me that their aim is to speak clearly to a very wide range of readers, not just students of physics or economics, but of other fields too. Indeed they hope for many readers outside the world of universities, people in financial institutions and companies and businesses of all kinds and sizes. Everybody in fact who is interested in or practises economics, and that is, I hazard the guess, almost everybody.

SAGR.: Is there much mathematics? It seems unavoidable. 
SALV.: Well you are right that there must be some, but the authors have designed their argument to make it accessible to those with only a basic mathematical training. Complicated mathematical formula are placed in a Mathematical side box, to one side of the main text, and those are indicated with a sign: $\alpha_{\beta}^{\gamma}$ icons.

SAGR.: Ah, that will be useful for me; very considerate.

Simp.: I also notice that there are 'Coffee Break' columns marked with and seeming to tempting digressions from the main subject of the book. That looked very interesting, though I still have my doubts about the main thesis of this work.

SALV.: Well that is forgivable since you have yet to read it, and when you do I have no doubt that your mind will throw up many questions as you get to grips with the work. You may find that dedicated support web site for the book answers some of these "http://www. econophysics.jp/books/ec". There is a great deal of additional information there, and of course you can contact the authors too.

SAGR.: Splendid, then let us meet again when we have read the book, and formed our opinions of it. Salviati, Simplicio.

SALV.: But first, shall we remind ourselves of the ubiquity of economic activities in our daily lives by dropping in to this pleasant looking inn and purchasing some refreshment before you return to your studies.

Simp.: For once, Salviati, you have said something with which I can wholeheartedly and completely agree. I do believe it is my turn, and fortunately I have my wallet with me. 


\section{Chapter 1}

\section{New Insights}

This book argues that the phenomena discussed within traditional economics can be approached fruitfully, arguably more fruitfully, by employing the concepts and methodologies of the natural sciences. In the present chapter we will describe the background to this claim, and some aspects of the contemporary situation in economics.

\subsection{A Scientific Approach}

What is the natural scientific approach, and why is it so powerful?

Descartes, of course, characterised science as the process of making ourselves free from any prejudice and dogma when seeking truth. Certainly, our capacity for thought is limited or distorted by the influence of religion, politics or, indeed, the received wisdom of established academic disciplines.

However, the fundamental principles of natural science warn us against these traps and require us to face natural phenomena without bias, and to resist the temptation to prematurely truncate our inquiries. Instead, we must ceaselessly root out error and improve our understanding. It was this attitude that enabled Galileo and his predecessors to overturn the prevailing Ptolemaic theory, and to provide a vastly improved model of the truth. Centuries of cumulative endeavour later we have a set of scientific views stretching from the imperceptible world of elementary particles right through to cosmology, the science of the universe as a whole. In between there is chemistry, biology and much else besides. The increasingly technological society we see around us is an outcome of the application of science and scientific method, and of ceaseless improvement in our conceptions of the world. But suppose that mankind had rejected the scientific viewpoint and approach and adhered to less disturbing ideas, to the comfortable traditional thought, for example, of those such as Galileo's contemporary Cremonini, ${ }^{1}$ who had refused to make

\footnotetext{
${ }^{1}$ Cesare Cremonini (1550-1631) was a conservative philosopher, and provided Galileo with the model for Simplicio in his Dialogue Concerning the Two Chief World Systems.
} 
observations through a telescope. Man would still be living in comparative intellectual darkness, with much of the potential of our minds, themselves the products of a long evolutionary process, unexploited.

Of course there is more to the history of science and scientific thought than Galileo, but his case is particularly instructive, and further comparison with the present situation in economics is, we think, helpful. Part of Galileo's revolution came about through the use of novel instruments, telescopes, to record previously unrecorded phenomena and aspects of phenomena. Similarly, students of economic behaviour may now use enormously powerful computing resources, and complex software embodying sophisticated mathematics, to collect, observe and analyse large quantities of economic data. It must be emphasised at this point that natural science is more than mathematics, though it is a wonderfully powerful language with which to describe nature. We do not doubt that mathematics will continue to play a hugely important, perhaps growing, role, but mathematics is just a means, not an end. To study natural or economical phenomena in order to exercise our mathematics is to fatally confuse task and tool. Just as it is in the other sciences, our objective in economics is to construct networks of true propositions that model the phenomena under consideration and extend our understanding of the causal processes at work.

Two goddesses are engraved on the reverse side of the Nobel prise medal for physics and chemistry. Nature is represented by the goddess "NATURA" emerging from clouds and holding a cornucopia in her arms. The veil covering her face is held and being withdrawn by the goddess "SCIENTIA", the spirit or genius of Science. This allegorical description of the process of science rings true for us. The sciences, the collective intellectual activity of many human generations, are gradually unveiling more and more of the natural world, and of all these research programs physics is the most rigorous and in some sense the most successful. Bearing in mind the features of economic phenomena, for example their fine-grained and intensely complex character, with micro-causes yield-

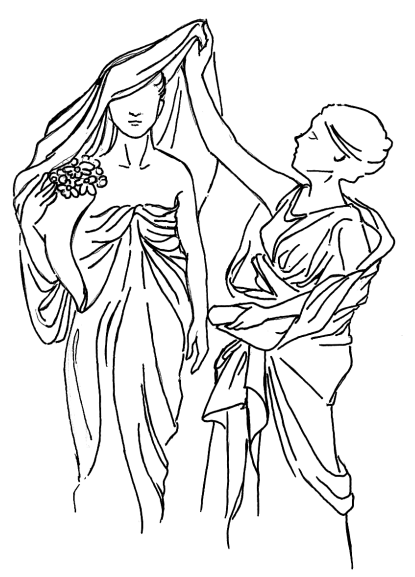
ing macro effects, it is not unreasonable to suppose that the methods of physics may give insight in this field too.

However, individuals are the minimum agents in economic and social phenomena, as consumers and workers, and since our behaviour is controlled by our emotions and thoughts, there is a possibility that we can find no fundamental laws describing these processes. Some may believe that it is impossible in principle to find any law at all. We may recall that Durkheim claimed that 
social facts can only be explained by other social facts. But science should not, through fear of failure, arbitrarily limit its scope, and in fact, physicists have already produced sufficiently valuable findings in economics to justify the expectation of more, and of more general insights. As a consequence there is an emerging and coherent inter-discipline, econophysics, which while not constituting independent academic departments is embodied in prominent international conferences and workshops.

\subsubsection{Science of Complex Systems}

Many readers may have heard of the "science of complex systems" and have some intuitive understanding of its work (Waldrop, 1992; Holland, 1996; GellMann, 1995; Holland, 1998). But what does it really mean in practice, and how does it relate to the subject of this book, the study of economic phenomena. Unsurprisingly, the science of complex systems is difficult to explain briefly, but one way to understand it is to take for a moment a different mental perspective from that of the principal philosophy behind natural science, namely the quest for fundamental laws and simpler and simpler explanations.

That is to say we can now see that many complex phenomena in nature are chaotic. Although the behaviour of such a system is derived from a simple law, it is unpredictable. A living thing, for example, should be regarded as a complex object, not simply as an assemblage of parts constituting atoms. That is, we cannot obtain a full picture of such a system by giving a detailed analysis of the individual system constituents. This concept began to gain ground amongst scientists in the 1980's, and is responsible for the emergence of the discipline now known as the science of complex systems.

This point of view is far from incompatible with that of traditional physics and its search for fundamental laws. These are complementary methodologies, a pair of wheels sharing the same axle, and both are necessary if we are to gain understanding of as broad a swathe of the natural world as we can at this time. This is as true for the investigation of economic phenomena as it is for any other aspect of the world. By combining a microscopic study of individuals and companies, one end of the axle, and a macroscopic study of outcomes of complex interactions among individual agents on the other end, we obtain a viable methodology with which we can make progress.

\subsubsection{The Emergence of Econophysics}

Only a decade has passed since the term "econophysics" was first used, and it is developing rapidly (Mantegna and Stanley, 2000; Aoki, 2002; Takayasu, ed, 2002; Bouchaud and Potters, 2003; Takayasu, ed, 2004; Aoki and Yoshikawa, 2007; Takayasu, ed, 2006; Aoyama et al., 2009). The pleasures of being involved in such a challenging and creative phase is extraordinary, but for those meeting the field for the first time it can be disorientating. Some reassurance can be gained from a glance back at the history of the relations between economics and physics, which is in fact long and close. 
For example, no less a figure than Léon Walras established the general equilibrium theory on the basis of the mechanical outlook of the world prevalent in 1860. The theory explains the balance between the demand and supply of goods that determines price by comparing this balance with a mechanical system consisting of a weight suspended from a spring. The forces of demand and supply correspond, respectively, to the gravitational force working on the weight and the restoring force induced on the spring, and the price so determined corresponds to the length of the spring in equilibrium. The agents in this theory thus represent consumers and producers of goods. ${ }^{2}$

However, the effectiveness of such an analogical approach is limited by the lack of both breadth and fine graining in its analytic texture. This matters because actual economic phenomena include such macro matters as the business cycle, and consequently we need to bear in mind the causal significance of the heterogeneity of economic agents, on the one hand, and economic fluctuations on the other. General equilibrium theory, which for instance describes a number of consumers via a single representative agent, is not able to account for dynamic effects in economic activity.

The discipline of statistical mechanics in physics offers understanding of macroscopic states of matter by employing microscopic information relating to atoms and molecules. It is a successful and structurally relevant model for efforts within econophysics to bridge between micro- and macroeconomics.

\subsection{Distributions and Fluctuations}

According to statistical data gathered over the last ten years, the Japanese economy is home to about 2.5 million companies. ${ }^{3}$ Additionally, Japan has approximately 67 million workers ${ }^{4}$ and more than one thousand financial institutions including city banks, local banks, credit associations, and governmental organisations. It is possible in principle to:

(a) list all of these economic agents,

(b) gain some grasp of the relationships among them,

(c) observe the financial and employment conditions of these companies,

\footnotetext{
${ }^{2}$ In economics, an agent is a constituent in a model used to solve an optimisation problem. Economic agents include, amongst others, households, companies, central banks, and governments.

${ }^{3}$ The total number of companies is based on a census taken by the National Tax Administration Agency. Other censuses by the Ministry of Internal Affairs and Communications, and the Ministry of Justice give different numbers. Accurate estimation of the number is said to be very difficult. For example, it may be overestimated by an over-thorough count which includes inactive companies or underestimated because the research is too cursory.

${ }^{4}$ This number is based on a census by the Ministry of Internal Affairs and Communications. The database covers workers over 15 years old and also includes persons without any employment at all.
} 
(d) monitor money flow between companies and banks and between banks themselves,

(e) store the entire data in real time.

This is a daunting task, but we have now well-developed computers equipped with superb CPU's, enormous amount of memory, and virtually unlimited data storage space. But would it be worth the effort? Such a database would certainly give us a perfect description of whole economy, but it would not make sense for us as outside observers because even a careful reading of the recorded data would tell us nothing about the rise and fall of companies, the main theme in this book. Clearly, some other approach is needed.

Let us move towards an alternative by reminding ourselves how companies are active in the production process. Each company buys materials and services from producers, adds value, perhaps by transforming them into other materials, services or products, and sells them on to other consumers, which can, of course, be other companies. In this process of earning money, the company employs its capital and labour along with its own creative ideas. We can see, therefore, that if we can trace the chain of money flows we will have described a very important aspect of the behaviour of companies.

To this end we can conduct statistical manipulations on the microscopic data relating to the 2.5 million Japanese companies and so extract coarsegrained representations. For instance, we might focus on the number of companies falling in the capital ranges of 1 million - 10 million yen, and more than 10 million yen. This kind of macroscopic view of the data in terms of statistical distributions will play a primary role in approaching the rise and fall dynamics of companies.

This is an idea akin to the approach adopted by statistical mechanics, but we are not claiming that the methods of this field are straightforwardly transferrable to economic phenomena. To gain understanding of any phenomenon, whether it is natural or socio-economical, we must distinguish between the topic's essential and inessential elements. Otherwise, we shall simply list all the data available, an act which yields little or no insight. Furthermore, we may encounter phenomena which do not lend themselves to that style of analysis. In fact, the rise and fall dynamics of companies are not random, and, as we will demonstrate from real data, certain dynamical patterns can be discerned, one of these being the distribution of corporate magnitudes. Indeed, it was Pareto, to whom we shall refer repeatedly in this book, who first pointed to this curious truth.

The first step in getting to grips with this matter is to recognise that the distribution of companies' sizes is not bell shaped, but significantly skewed. The normal or Gaussian distribution, familiar from general statistics, is a typical example of the bell-shaped distribution, and if company sizes were distributed in the normal form, we would find a majority of companies of average size with a few exceptional companies of very small or very large size. But study of the data shows that, in fact, companies are classified into two groups, a small number of giant companies and a large number of small and 
medium-size enterprises. If we remove the giants and examine the remaining companies we find that they can classified into two groups, the very large and the rest, a procedure that can be carried out repeatedly. This real-world distribution is characterised by a self-similar hierarchical structure, an aspect of the finding with which some readers may already be familiar. However a nontrivial point is that the distribution obeys a specific form of distribution, the power-law distribution, details of which are discussed in Chapter 2.

Of course, distributions are just collections of snapshots of living companies. If we are hoping to shed light on the dynamics behind these distributions, we need to analyse any fluctuations, such as variations in capitalisation, which reflect the driving forces of production activity. In a static model where everything is balanced without fluctuations, there would be no dynamism at all, and it would be impossible to understand how a particular pattern is brought about and under what conditions the pattern is destroyed. However, where there are fluctuations, and they are invariably present in real-world cases, they are very revealing, and in Chapter 3 we will show that such fluctuations have a distinctive pattern giving rise to a specific distribution.

As has been remarked, analyses of real data shows that there are clearlyvisible patterns in the distributions and fluctuations relating to companies, and that these are independent of variables such as country and time. The existence of such universality in such phenomena is extremely surprising from the perspective of economics, and encourages the use of the methodologies of natural science in their analysis. But the difficulty of the problem that faces

\section{Vilfredo Pareto}

Vilfredo Pareto (1848-1923) was an Italian economist. He was born in Paris to an Italian father and a French mother, and raised in that city before returning to Italy to study Mathematics, Physics, and Engineering at the University of Turin. Subsequently he worked as a civil engineer for the Italian state railway company and then for an iron works, of which he was for ten years the director. During this time he became a fierce proponent of free trade and minimal government regulation. This led him to intense political activity, and then to a new career in economic studies.

Under the influence of the neo-classical economist Leon Walras, he became a professor in economics in the University of Lausanne at the age of 46. His book Cours d'économie politique (1897) describes the power-law that he both proposed and fiercely defended against criticism. Pareto's power-law is one of the central components of the Econophysical studies of companies and other agents in the real economy, not to mention many other social and natural systems where self-similarity is observed. 
us should not be underestimated, for as we try get to grips with the dynamics of the growth and failure of corporations, we will find that we are seeking an understanding of the interactions between agents, that is, of an economic network formed by the enormously complicated pattern of relationships between agents.

\subsection{Are Networks Complex?}

The power-law distribution, which is observed ubiquitously in nature, is a critically important concept in this book, and has in recent years been used to great effect in network science, a relatively new science dealing with a wide variety of phenomena, ranging from the microscopic, for instance in biology where it discusses gene networks, metabolic networks, and the interaction network amongst proteins, right through to the macroscopic, where the internet provides us with typical examples (Watts, 1999, 2003; Barabási, 2003; Buchanan, 2003; Caldarelli, 2007). For instance, researchers are also interested in communication networks formed by providers and companies, and the linking structure of web pages, social networking services (SNS), the trackback network of blogs, and so on. Other examples might include coauthor relations amongst researchers, and friendship networks among football players.

At first glance it may be difficult to believe that the members of such an apparently heterogeneous collection of networks have any common features, but in fact there are several, a fact that points towards the existence of some universal law behind the formation processes of networks. On the other hand, it is also true that each network has its own characteristic features, and one of the challenges confronted by network science is to explain how these facts co-exist.

The application of network science theory to economic phenomena is described and argued for in detail in Chapter 4, but for the time being few readers will object to the claim, "Economy is a very large network consisting of economic agents directly and indirectly linked to each other". Interestingly, although such a proposition seems unobjectionable, almost commonplace, the idea of studying economic systems from a point of view of networks is a recent one. For instance, the pioneering book written by economists with this perspective was published only in 1984 (Piore and Sabel, 1984).

Here we pay our special attention to growth and failure of companies interacting with each other over various kinds of business networks. Such intercompany networks underlying the dynamics of companies include mutual shareholding relationship, linkage between companies established by interlocking directorates, transaction relationships between sellers and buyers, and collaborative innovation emerging by means of joint applications for patents.

The authors began their study of business networks around 2000, and at that time even at international conferences there were very few presentations on this topic. However, since that time the number of papers on this theme has 
been increasing rapidly, and network science is now one of the most important key terms at such gatherings. This dramatic change is a clear indication of the growing understanding that recent developments in network science are relevant to all fields, with economics being no exception.

Indeed, there are points of very close contact between economics and network science, for example the environment surrounding companies is a rapidly changing one, and accordingly the relationship between companies undergoes a dynamic influence. Consequently, it is dangerously misleading to focus our attention exclusively on a target company while neglecting its relationship to other companies. Taking a broader view will reveal new aspects to what is actually happening in the industrial economy, a topic that we will take up in the next section.

\subsection{Change of Environment Surrounding Com- panies}

In recent years industry has made a marked shift from the vertical integration structures to those characterised by the horizontal division of work. As a result, managers and analysts have begun to recognise just how important the formation of networks are for corporate competitiveness. Environmental changes of this kind occurred in the electric machinery and automobile sectors, two major industries in Japan, from the late 1980's onwards. ${ }^{5}$

\subsubsection{Outline of the Japanese Electric Machinery and Automobile Industries}

Before the middle of the 1980's, conglomerates in the electric machinery sector, for example Hitachi, Toshiba, Mitsubishi Electric, established their positions in the Japanese economy. They produced the whole range of electric machineries. For instance, their product range spans appliances, electronic components, such as liquid crystal panels and DRAM chips, electric power equipment, such as power generators and power grid systems, computers and system integration, such as a bank's mission-critical systems, to telecommunication equipments, such as routers. Their business strength came from their wide product range, because the phase and period of business cycle for one kind of product is different from those for another kind. They had grown steadily for a long period without experiencing serious poor performance. It is well known today that a major origin of high profitability in the 1980's was the export of DRAM chips to the United States of America (USA).

On the other hand, the automobile industry established a unique strength in production systems through continuous effort for decades. The industry consists of several automobile manufacturers, such as Toyota, Nissan

\footnotetext{
${ }^{5}$ Business environmental changes in global economy are explained from the viewpoint of USA in Dertouzos et al. (1989) and Berger (2005).
} 
and Honda, and a very large number of auto-parts manufacturers, producing transmission, brakes, electronic engine controllers and other components. Most of the auto-parts manufacturers are located near the factories of automobile manufacturers. They supply various auto-parts immediately to the factory requesting parts. This supply method brought a very high efficiency to their production system. Automobile manufacturers made various small- and medium-sized cars in Japan, and exported them mainly to the world biggest market, that is USA.

In the middle of the 1980's Japan came under sharp criticism from USA for its continuously growing trade surplus. A long sequence of repeated negotiations between the two countries eventually created a new economic context for Japanese industry, and after the Plaza Accord signed by the economically developed nations, the relative value of the yen increased rapidly, with the consequence that automobile manufacturing plants were relocated in USA At the same time, various political schemes were devised to increase domestic demand. After the termination of the Cold War, however, political interventions of this kind were rejected, and were ultimately succeeded by an era of deregulation. This sequence of policy changes had consequences for industrial structure, and business networks are key to understanding these changes.

\subsubsection{The Electric Machinery Industry}

In the 1980's Japanese companies survived intense competition from American competitors in the manufacturer of audio-video equipment such as video recorders and televisions. This was also broadly true for companies making components of personal computers, such as liquid crystal panels and DRAM chips. As a result, USA government accused Japan of dumping DRAM chips, and forced Japan to monitor their export prices by concluding the JapanUSA Semiconductor Agreement (from 1985 through 1995). In the process of increasing production of liquid crystal panels and DRAM chips, Japanese companies built their plants in Korea and Taiwan, a decision motivated in part by the desire to avoid trade conflict. To reduce possible risks in these projects the Japanese companies asked local companies to invest in the business and in return offered technical expertise. This period of activity overlapped to some degree with that during which the Japan-USA Semiconductor Agreement was effective. The point to emphasise here is that Japanese companies changed their industrial structure from vertical integration to a horizontal division of work by switching from self-manufactured components to those out-sourced through collaboration with companies abroad. It should also be remarked that the distribution of process units also entailed the leakage of production technologies.

Although Japanese companies became front-runners in the 1990's, this golden age did not persist for long, as companies were exposed to aggressive pursuit by Korean and Taiwanese companies, and a price drop due to over production. Furthermore, the Japan-USA Semiconductor Agreement seriously damaged Japanese companies and at present Japan has only one 
manufacture for DRAM.

Putting aside the subject of specific components for personal computers, there are other more general problems regarding general environmental or contextual change in the computer manufacturing sector. Until the 1980s the computer world was largely a closed system, in which Japanese companies were able to retain a high-profit business in relation to IBM-compatible mainframe computers for the mission-critical systems of banks and other large financial institutions. This really was an age of vertical integration, but after the end of the Cold War in 1990, a new period of open systems emerged, one characterised by personal computers and networks. At that time Japanese companies failed to gain control of the standardisation of the CPU for personal computers so that they had to follow an industry standard determined by companies in the United States. The simple assembly of components and the localisation of software packages were not sufficient to permit Japanese companies to take advantage of cumulative technological development by making further progress, and the superiority of Japanese companies has consequently been gradually eroded.

The explosive development of the internet led to a coming of age, if not quite maturity, in the late 1990's, and, combined with deregulation policies, has radically transformed the industrial structure of communication equipment businesses from vertical integration to horizontal division of work. The new wave has all-but destroyed the industrial cluster consisting of NEC, Fujitsu and Hitachi, once called the "Denden family", and the Nippon Telegraph and Telephone Public Corporation, NTT, which exerted near complete control over this family, which it also supported. Even Japanese companies which manufacture telecommunication equipment for open systems, such as routers, are being out-competed. In contrast USA has a number of highly-profitable fabless companies ${ }^{6}$ such as Cisco, which can respond promptly to market needs by simply switching manufacturer. This remarkable outcome is a clear manifestation of the strong causal relationship between network formation and competitive powers.

In addition, the electric power equipments division is undergoing drastic changes due to deregulation. The electric power industry is typical, having been government controlled until the 1990's. Companies making electric power equipment were able to main high profit margins via their relations with the power companies. However, deregulation of the energy market, which started around 2000, has begun to change this intimate relationship. ${ }^{7}$ Some equipment makers even have their own power plants to sell electricity to power suppliers, and in future it is expected that the formation of business networks amongst generators and suppliers will lead to reductions in the price of elec-

\footnotetext{
${ }^{6} \mathrm{~A}$ Fabless company is an equipment manufacturer that does not have its own manufacturing capability for electronic components, which is known as a fab.

${ }^{7}$ USA experience of the deregulation of electric power is instructive, since it allowed the emergence of Enron, the notorious collapse of which due to a large quantities of improper transactions is one of the defining corporate scandals of our time, and has focused discussions of corporate governance.
} 
tricity.

\subsubsection{Automobile Industry}

Like the electric machinery sector, the automobile industry has also undergone global reorganisation as a consequence of a trade conflict between USA and Japan, notably Japanese companies were prompted to build manufacturing plant in the United States. However, it was the acquisition of Chrysler by Daimler-Benz that triggered the most important phase of global reorganisation. The fact that size matters for survival was widely recognised, as might be guessed by the term "Four Million Club" to refer, somewhat enviously or complacently, to those automobile manufacturers produced over four million vehicles per annum.

However, looking through the industrial structures of Japan, we observe no fundamental change that excludes certain of the automobile manufacturers. Similarly, in USA we observe an industrial system established with the cooperation of the Japanese automobile manufacturers and auto-parts manufacturers. Indeed, Japanese companies have now caught up with USA companies as regards sales numbers in spite of the fact that these American companies retain a strong influence on the market. This is partly because these USA companies must incorporate very large costs of welfare, including pensions and medical payments for retired employees, into the prices of cars. By comparison Japanese companies enjoy relatively low costs for welfare, in addition to efficient production technologies, and can therefore increase their market share and maintain a healthy profit margin. Concerns with regard to energy security and environmental pollution have provided a tailwind for those Japanese companies excelling in the production of economical small- and medium-sized cars. By contrast, Korean companies are still in the catch-up stage on this matter.

The key production technology keeping Japanese companies internationally competitive is just-in-time production which makes each process hold inventory to a minimum (Ōno, 1988). The Kanban system is key to the realisation of this efficiency, and is used in the following way. A Kanban delivery slip is transferred by the manufacturer to the post-process stage together with manufactured goods. Once the goods are used in the post-process, the Kanban is returned to the preceding manufacturer process as a purchase order. Thus, in each process goods are only manufactured once authorised by a Kanban, creating a chain reaction of production with minimum inventory.

Just-in-time production is based on a business network, in which vertical integration for key components such engines and automobile bodies coexists with a horizontal division of work for peripheral components such as electronic parts. Formation of a suitable business network is the key to efficient and flexible production. 


\subsubsection{Industrial Structures and Business Network}

In the last twenty years, the electric machinery industry in Japan drastically changed its shape in ways which will be familiar from the other industry examples discussed above. Instead of self-manufactured components they are now using out-sourced components to produce computers, communication, and other types of equipment. This change in industrial structure, together with a move towards an open system due to deregulation, has confronted electric machinery companies with a major alteration in relationship with other companies in the sector. Generally speaking, this has required companies to regard themselves as a part of the whole economy, not as a single entity isolated from the system.

On the other hand, the automobile industry continues to develop business networks in an environment made up of a mixture of the two industrial structures. Broadly speaking, vertical integration is used for autobody and core components such as engines, while horizontal division of work is used for peripherals such as electronic components. However, strengthening of the just-in-time production methods, with the aim of keeping Japanese industries internationally competitive, clearly requires elucidation of the business network structure. Furthermore, a case study of the successful handling of major accidents shows that the business network plays an important role in quickly recovering from disasters.

To shed light on these issues in the industrial economy, we thereby have to elucidate what network is formed by companies through their linkages and how they interact with each other on the network, and it is expected that methods developed for analysing complex networks and agent-based simulations will be very useful, as demonstrated in Chapter 5. Perspectives for possible applications of the basic insights obtained in the previous chapters to practical business are given in Chapter 6. Topics such as business strategy development methodology, the management of the propagation of credit risk, and innovation of business models, are also explored.

In the following chapters we will explain, using many figures, what analyses of real data and simulations teach us about the statistical life and death of companies, and also the relationships between companies. We are all facing a great new wave of methodological opportunities for the understanding of economical phenomena, and have designed our book to serve as a messenger of some parts of what we believe will be an intellectual revolution. 


\section{Powers of ten}

At many points in our text we use large numbers. In some cases, for example money, our intuitive understanding, of one million dollars say, is adequate, but it is not always so, and in some cases the quantitative comparison of two large numbers can be opaque or cumbersome. For example, faced with two numbers it is not always immediately obvious how many times greater one is than another. Using exponent expressions such as $10^{6}$ dollars is much more convenient in these cases. Within a Western system properly known as the "short scale" special names are assigned to large numbers in units of $10^{3}$ as shown below:

$$
\begin{aligned}
& \text { thousand }=\quad 1,000=10^{3} \\
& \text { million }=\quad 1,000,000=10^{6} \\
& \text { billion }=\quad 1,000,000,000=10^{9} \\
& \text { trillion }=1,000,000,000,000=10^{12}
\end{aligned}
$$

This continues up to vigintillion $\left(=10^{63}\right)$. Interestingly, in the East the basic unit is $10^{4}$, giving, in Japanese, "Man" $\left(10^{4}\right)$, "Oku" $\left(10^{8}\right)$, "Chou" $\left(10^{12}\right)$, "Kei" $\left(10^{16}\right)$, and so forth. "Muryotaisu", the largest unit for numbers currently used in Japan is $10^{68}$, which makes $10^{72}-1$ the largest number that can be described by words.

It is curious to note that there are other special names for large numbers, and that these do not form part of a sequence, and therefore cannot be called "units" in a strict sense. For example, Richard P. Feynman once remarked that "There are $10^{11}$ stars in the galaxy. That used to be a huge number. But it's only a hundred billion. It's less than the national deficit! We used to call them astronomical numbers. Now we should call them economical numbers." He, however, must have meant "debt" not "deficit", because the latter is about $5 \times 10^{9}$ (in USA dollars, 2008) while the former is about $10^{13}$ similarly. Thus we propose that:

$$
1 \text { Feynman economical number }:=10^{13} \text {. }
$$

In the microscopic world, $10^{-13} \mathrm{~cm}$ is 1 Fermi (Yukawa), which is approximately the radius of the proton. Therefore, 1 Feynman is the inverse of 1 Fermi (Yukawa), so to speak.

Incidentally, there are other names for extremely large numbers, such as googol $\left(10^{100}\right)$, centillion $\left(10^{303}\right.$ or $10^{600}$, depending on which side of the Atlantic you are), and googolplex $\left(10^{10^{100}}\right)$. In physics, a large number we encounter is the total number of protons in the observable universe, which is known as the Eddington number, and is approximately $1.57 \times 10^{79}$.

No economist should want to encounter numbers beyond 1 Feynman. 


\section{Chapter 2}

\section{Size Distribution}

By looking at a wide conspectus of companies, rather than individual organisations, a curious and somewhat mysterious pattern becomes evident in the rise and fall dynamics.

As a preliminary to examining the dynamics of group of companies, we will first explain what we mean by the size of a company, and then introduce the important concepts of flows and stocks, and several tools necessary for examining the distribution of company sizes.

We will find that among "giant" and "dwarf" companies, the size distribution of giants obeys Pareto's law. We will also see that the same law is also found for flow quantities such as individual incomes, and we will touch upon its connection with inequality in society and how it is linked to macroeconomics.

We can now turn to the strange world of company dynamics.

\subsection{Preliminaries}

\subsubsection{Flows and Stocks}

Companies are economic agents that buy materials and services, and create added value by utilizing their own resources, and earn money by selling the products. Thus, the key to understanding company behaviour is a clear understanding of money flows. Companies raise money, and use this capital to obtain raw materials, employees, and facilities, and then by using these resources add value to products and services, which are then sold to obtain more money.

A company is usually legally obliged to communicate its financial situation to shareholders and creditors through audited accounts and statements, which are by and large in the public domain. Such sources of information are valuable source of information about company behaviour, but in using such measurable variables, it is critical to recognise that flows and stocks have very distinct physical dimensions. For example, let us take a person's savings account, 
where both deposits (inflows) and payments (outflows) occur. The current balance is a stock, and is a quantity measured at a point in time and in monetary units (yen, pounds, dollars, etc.). On the other hand, the payments made in a particular month constitute a flow and, are measured in units of yen per month. Stocks and flows are therefore differentiated by time.

While a flow is a change of stock, stock itself is a total-sum of flow in the past. Consequently, there's more to describing the financial standing of a company than examining stocks or flows on their own. The same is true of a national macro economy, where GDP (Gross Domestic Product) is a flow and National Balance Sheet is a stock.

For a company, flow quantities including sales, expenditure, and operating profit are measured in the Income Statement, and stock quantities such as capital and debt are measured in the Balance Sheet.

Now, the size of a company is a measure of available money and can be measured from stocks like capital or debt, or from flows like sales or operating profit. The number of employees is also another good measure of size. On the other hand, no single variable is adequate for determining the size of a company. The company with the largest sales is not necessarily that with the greatest capital.

However, by examining the size of a large number of companies from various angles, we find that the fundamental properties remain the same regardless of the variable used, stocks or flows. This may not be surprising since all these stock or flow variables are induced by the money dynamics of the company.

In order to deal with a group of companies, such those in a business sector (energy say, or food processing), or the companies within a nation state, it is critical to use data that is either as exhaustive as possible or has been obtained from consistent examination of carefully selected samples. Otherwise, the analysis may produce misleading results with regard to the distribution and behaviour of the population (see Section 2.3.3). Even if the sampling is wellplanned, it is quite common for only summarised statistics to be published, and individual data is available only to a limited number of researchers, and consequently the dynamics of companies are hidden. One way round this is to use information from income and corporation tax, and this is the route that we adopt here. ${ }^{1}$

\subsubsection{Size Distribution and Pareto's Law}

If it were possible to obtain all the flow and stock quantities for all companies and individuals at all times, a complete description of these economic agents would be obtained. However, such a description would be almost meaningless,

\footnotetext{
${ }^{1}$ It may be objected that tax data are potentially prone to error, since actual income and declared income might vary. However, it can be argued, and we think persuasively, that listed companies and high-income individuals will file accurate tax forms since the penalties, both legal and reputational, are considerable. Furthermore, statistical analysis reveals some outstanding phenomenological laws, which may be a reflection of truths of a still deeper interest, and this in itself makes the data worth examining.
} 


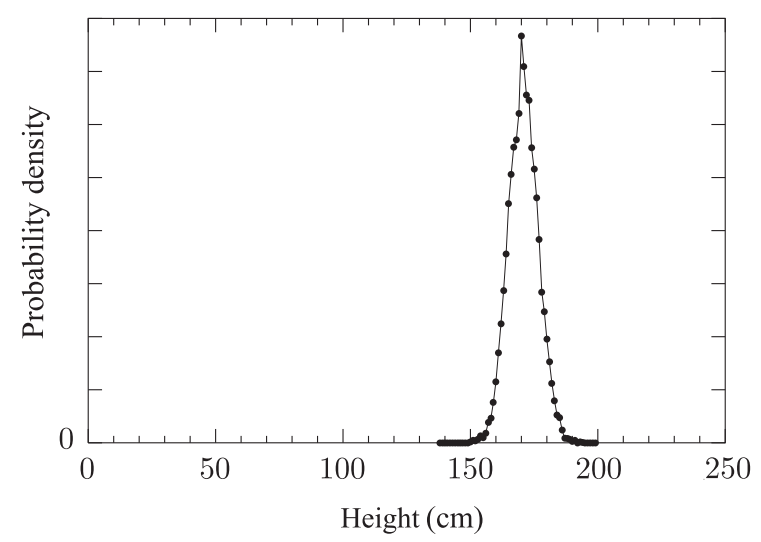

Figure 2.1: Probability distribution of human height (High school senior male students in Japan).

and what we require are statistically-sorted facts. That is to say, instead of a complete list of the capital status of a million companies, it would be more useful to obtain macroscopic descriptions, such as the number of companies with capital between one hundred million and one billion yen, or how many companies have capital in excess of a billion yen. Briefly, distributions are the key to understanding the dynamic properties of the way in which companies rise and fall.

As we have mentioned in Section 2.2, this is identical to the methods used in statistical mechanics for analysing microscopic and macroscopic physical states. Since the distribution of company sizes is extremely surprising we will first introduce some appropriate tools for handling these, and make comparisons with other distributions.

The height of male students in the third year of Japanese high school in 2005 is plotted in Figure 2.1. We denote the height by $x$ and the frequency of people with height between $x$ and $x+d x$, where $d x$ is a small number, by $p(x) d x$. For the sake of brevity we will refer to the function $p(x)$ as a probability density function $(\mathbf{P D F}) .^{2}$

Figure 2.1 is a histogram with a abscissa graphing the height $x$, and ordinate graphing the frequency $p(x) d x$. Adding all the frequencies over the

\footnotetext{
2Strictly speaking, the term "PDF" applies to cases with continuous variables. In this case, since the data is tabulated, the height variable is essentially discrete. The same is true in economics, where the variable $x$ could be money or number of people and hence is often discrete. In such cases, and strictly speaking, the relevant term is "probability mass function". However, the difference is not that important and because the scale of the variable is so large compared to the minimum unit, i.e. a million pounds to $1 \mathrm{p}$, and also because translating all equations and discussions from continuous variables to discrete variables is straightforward. For these reasons we use the terminology for continuous variables throughout this book.
} 


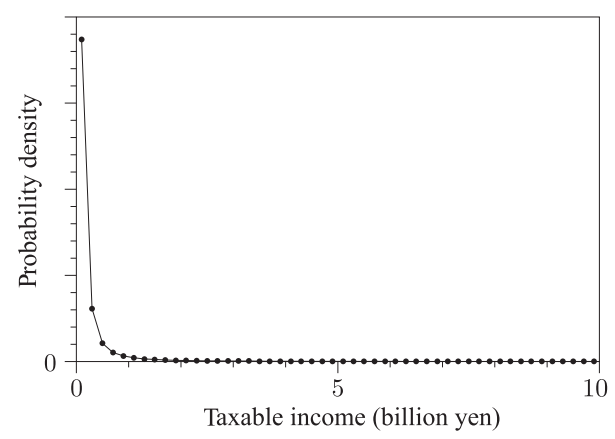

Figure 2.2: PDF of companies' declared income.

range yields a result of 1 , which means that the total area of the histogram adds up to 1.

Note that in this figure most of the individuals are distributed around about $170 \mathrm{~cm}$ in height, and in spite of individual differences no-one is $10 \mathrm{~cm}$ or $500 \mathrm{~cm}$ high.

This kind of bell-shape, which is centred around a peak and rapidly decreases away from it, is quite common, and the so-called "normal distribution" is a representative instance. This is a very standard and untroubling distribution.

\section{$\widehat{\alpha_{\beta} \gamma}$ Normal Distribution \\ The Probability Density Function (PDF) of a normal distribution $p^{(\mathrm{n})}(x)$ is the following: \\ Here, $\mu$ is the mean and $\sigma^{2}$ is the variance.}

By comparison, the distribution of company sizes exhibits striking properties. Take the declared income of companies, an area in which we can get access to data from nearly all the companies with positive income. Figure 2.2 is the PDF plot of the top seventy-eight thousand Japanese companies in 2002, represented in a manner similar to that used above for human height. It can readily be seen that this distribution is widely spread. The largest income is the six hundred and eighty trillion yen of the Toyota, which is far too large to be included in Figure 2.2. The smallest income in this data is about twenty million yen. Although there must be companies with an income smaller than this, we can say with confidence that the income of companies differs by at least fifty thousand times. This makes the distribution in Figure 2.2 rightskewed. A better way to describe this characteristic of the distribution is to 


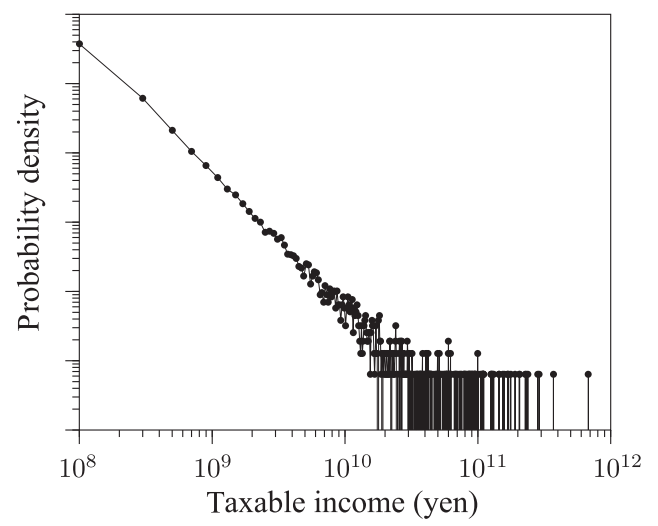

Figure 2.3: Double-logarithmic plot of PDF of companies' declared income.

say that it has a long tail. ${ }^{3}$ This sort of long tail is sometimes also called a "fat" or "heavy" tail.

The total of income gained by all the domestic companies is limited. In view of the fact that there is a huge disparity between companies it may not be surprising that a small number of companies engrosses a large share of income. However, there is a pattern in this plot. Fig. 2.3 is a double-logarithmic version of Fig. 2.2. This plot shows that PDF can be approximated by

$$
\log p(x)=-\alpha \log x+c,
$$

where $\log$ denotes the natural logarithm and $\alpha$ and $c$ are constants. This approximation is valid in a very wide range, from one billion to several hundred billion yen. The equation above may be rewritten as,

$$
p(x)=C x^{-\alpha},
$$

where $C=e^{c}$. Thus this distribution obeys a power law, and the constant $\alpha$ is the exponent of the power-law.

Careful examination shows that the distribution in Figure 2.3 is somewhat shaky in the range $x>10^{10}$. In making this histogram, we have divided the abscissa of $x$ into small equal-sized segments and have counted the number of companies in each segment. As $x$ becomes larger, the size of the segments becomes smaller in proportion and there are many segments without data, causing a rough appearance. Naively, it might be supposed that this could

\footnotetext{
${ }^{3}$ It will be as well to note in passing that the long-tail described here is not directly related to the recently popular notion of "long-tail" in business. We will elaborate on this in Section 2.5 .
} 
be avoided if larger segments were chosen. One might also choose variable sized segments that become larger as $x$ increases, in order to avoid segments with zero entry. For example, we might choose segments with size equal to $\log x$. However, optimised choice of segments remain as a technical problem, and the roughness remains no matter what. Moreover, by taking segments of a larger size, we lose some of the information in the original data. These two problems conflict with each other: smaller segments allow us to keep detailed information but leads to roughness in the results, while larger segments leads to a smoother PDF but results in a loss of information. Selecting the right size for the segments, in order to achieve a reasonable balance, is an extremely frustrating technical problem.

We can work around this problem by using a cumulative distribution function, or CDF. ${ }^{4}$ The $\operatorname{CDF} P_{>}(x)$ is defined by the following:

$$
P_{>}(x)=[\text { The rate of data with a value larger than } x]
$$

By this definition, $P_{>}(\infty)=0$, and if there is a lower bound $x_{0}$ for $x$, $P_{>}\left(x_{0}\right)=1$, as all data has $x$ larger than $x_{0}$, it is also evident that $P_{>}(x)$ is a monotonically decreasing function of $x$.

$\mathrm{CDF}$ is related to $\mathrm{PDF}$ as follows:

$$
P_{>}(x)=\left[\text { Total sum of } p\left(x^{\prime}\right) d x^{\prime} \text { with } x^{\prime}>x\right]
$$

That is, $P_{>}(x)$ in an integration of $p(x)$. From this we find from the equation on page 19 that the power-law distribution has the following CDF:

$$
P_{>}(x) \propto x^{-\mu} \Longleftrightarrow p(x) \propto x^{-\mu-1}
$$

The power-exponents are related by $\alpha-1=\mu$.

\section{$\alpha_{\beta}^{\gamma}$ PDF and CDF}

The definition of the CDF above can be written as follows using the integral.

$$
P_{>}(x)=\int_{x}^{\infty} p\left(x^{\prime}\right) d x^{\prime} .
$$

Therefore, the PDF is obtained from the CDF by differentiating it:

$$
p(x)=-\frac{d P_{>}(x)}{d x} .
$$

This leads to the relation between the exponents $\alpha=\mu+1$.

In practise, the CDF of data can be obtained as a rank-size plot. Let us say we have $n$ data, $x_{1}, \ldots, x_{n}$. We sort them in decreasing order and denote them as $x_{(1)} \geq x_{(2)} \geq \cdots x_{(n)}$. The $i$-th rank data is $x_{(i)}$ and there are $i$ items of data with a larger size, $x \geq x_{(i)}$. Therefore, if we plot them with the

\footnotetext{
${ }^{4}$ This is also known as a cumulative distribution or cumulative probability.
} 


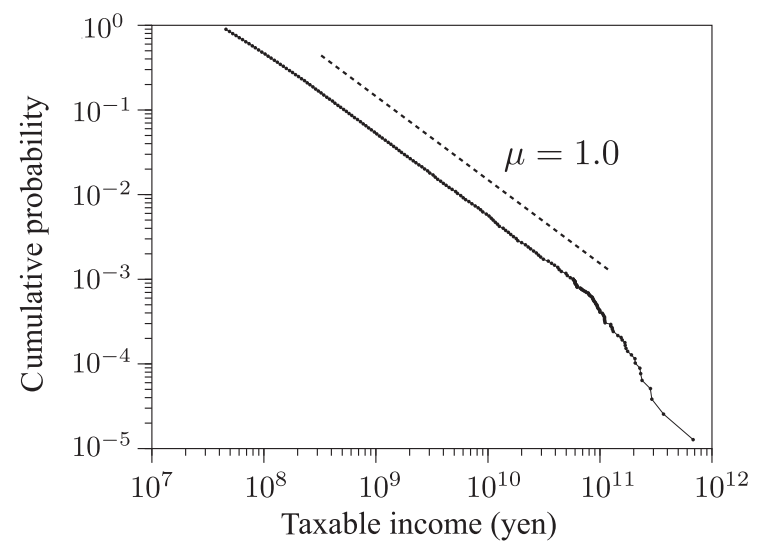

Figure 2.4: Double-logarithmic plot of CDF for companies' declared income.

size $x$ on the abscissa and rank divided by the total number of data $n$ on the ordinate, it yields the plot of the observed CDF.

Or, one may simply plot the rank on the ordinate, we obtain the CDF multiplied by $n, n \times P_{>}(x)$.

This kind of rank-size plot does not suffer from the problems we encountered in making histograms, so we don't need to worry about the size of the segments, and we get to keep all the information. The rank-size plot is, therefore, an ideal tool for examining these distributions.

Sometimes the ordinate indicates the size and the abscissa the rank, with their roles exchanged. Also, the data could be sorted in increasing order, not in decreasing order. In that case, we would be looking at a variation of the CDF:

$$
P_{<}(x)=[\text { Rate of data with its value smaller than } x],
$$

which is related to our original CDF by the following:

$$
P_{<}(x)=1-P_{>}(x) .
$$

Therefore all these approaches are in some sense looking at the same property, but from different angles.

Figure 2.4 is a rank-size plot of the companies' declared income. According to this, from $x=10^{8}$, the income ranges from a hundred million yen, to $x=10^{11}$ a hundred billion yen, the power law evidently applies with the power exponent very close to $\mu=1.0$.

A number of natural, social, and economic phenomena obey the power-law distribution, for example the magnitude of earthquakes, the size of craters on the moon, the strength of solar flares, and the size of fragments of shattered 
objects. Amongst social phenomena examples might include the size of cities, the number of references to academic papers, the frequency of appearance of words, and individual incomes.

Individual income was investigated first by Vilfredo Pareto in 1896 (see the column in Chapter 1), and made a huge impact on the issue of inequality in economics (Pareto, 1896). The power law for the size of cities was noted first by Felix Auerbach in 1913 (Auerbach, 1913), while the size of companies was studied by Herbert Simon and many other researchers. G. K. Zipf's book in 1949 studied long-tailed distributions in a wide variety of social phenomena and human activities and had a considerable influence in the natural science and the humanities, amongst the best known being Zipf's law concerning the frequency of English words (Zipf, 1949).

However, it is important to be aware that while some phenomena appear

\section{The Size of Cities}

The choices made by individuals with regard to where they live, in large or small cities, are certainly influenced by variations in individual life story and local culture and history. Nevertheless, human societies resemble and often exhibit mysterious and surprising self-organizing phenomena. The chart below plots the population of the cities and towns in the northern Japanese island of Hokkaido, based on data obtained from the censuses of 1980 and 2005 .
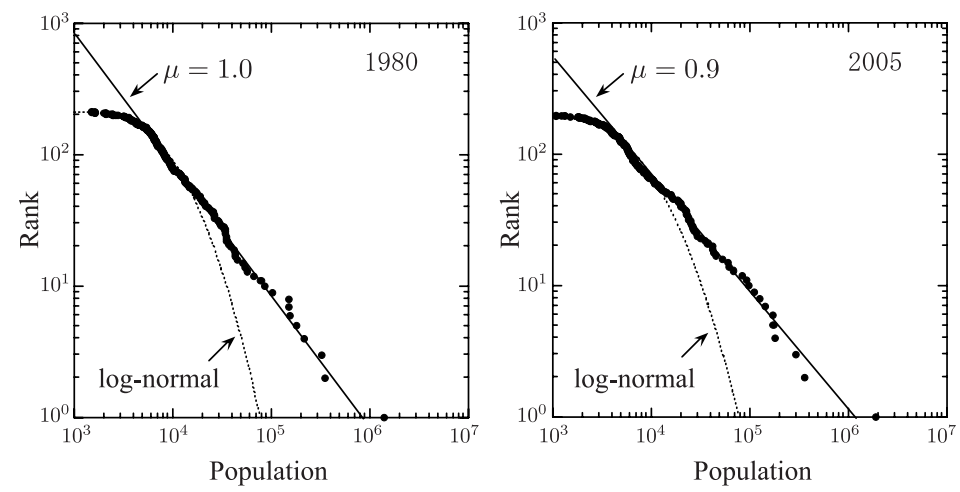

Even though there only around two hundred samples a power law is clearly evident in the tail (the fit to the logarithmic normal distribution is also represented in the figure). A rough estimate of the power exponent for 1980 would be $\mu=1.0$, but $\mu=0.9$ in 2005 suggesting that population has become more concentrated in large cities as a smaller value for $\mu$ indicates a greater number of larger cities. As we explain in Sections 2.4 and 2.6, a value for $\mu$ smaller than 1 indicates the oligopolistic phase. 
to obey a power law at first sight, close examination reveals that in fact they do not.

In quite a number of power law cases the power exponent $\mu$ is somewhere between 1 and 3. A smaller value for $\mu$ indicates a broader distribution, and thus the existence of larger quantities. In this sense, the power exponent $\mu$ is a measure of inequality in the region where the power law applies. We will elaborate on this point in Section 2.4.

As is well known, the power law is often called Pareto-Zipf's law, or simply, Pareto's law. In particular, in the case of $\mu=1$, and in relation to phenomena such as city size or frequency of words, it is commonly referred to as Zipf's law. This is no accident: $\mu=1$ is one of the most important of power laws, a point we will elaborate in Section.2.6.

Hereafter in this book, for brevity we shall refer to the general power law as the Pareto's law, or as the Pareto distribution, and its exponent as the Pareto exponent.

\subsubsection{Other Distributions with a Long Tail}

It should be noted that the term "Pareto distribution" is based only on the behaviour of the distribution in the asymptotic region, where its variable $x$ is large. Thus it covers a range of distributions, which differ in their functional behaviour in the small-to-medium range, and some of these will be explained in a later section. Thus, the "Pareto Distribution" in this sense is a generic name.

The Pareto distributions are not the only long-tail distributions, a particularly well-known instance being the log-normal distribution, which is, in essence, a normal distribution in $\log x$. Another important long-tail distribution is the Tsallis distribution (Tsallis, 1988), which has some desirable mathematical properties, although we will not use it in this book.

As we have argued, the Pareto distribution is a class of distribution with a long-tail, and is used most extensively due to the following reasons:

- Power-law behaviour is apparent in a wide variety of data.

- The Pareto Distribution has only one (significant) parameter, the Pareto index (Farmer et al., 2005).

- The processes that induce power-law distributions are well understood.

Of course, it is important to make a careful examination of the dynamics behind the phenomena in question and to determine what kind of distribution is in fact most suitable for understanding its true nature.

\subsection{Distribution of Personal Income}

We will digress here to deal with the issue of personal income. This was studied in earlier work by some of the present authors on the basis of a very 
large Japanese data set which allowed the discussion of this distribution in detail, with particular emphasis on issues of inequality and their relation to the macroeconomic situation (Aoyama et al., 2000). A lack of similar data in other countries seems to have prevented work of similar accuracy before and since (but if any reader knows of comparable data we would very much like to hear of it). Because of its apparently unique status this data has become an important corner-stone of research on the subject of personal income.

While reading this section keep your own income in mind, to put the study

\section{$\alpha_{\beta}^{\gamma}$ Log-normal distribution}

The PDF of a log-normal distribution $p^{(\ln )}(x)$ is defined in the range of its variable $x$ from 0 to $\infty$ as follows:

$$
p^{(\ln )}(x)=\frac{1}{\sqrt{2 \pi} \sigma} \frac{1}{x} \exp \left[-\frac{(\log (x / a))^{2}}{2 \sigma^{2}}\right] .
$$

This is obtained from the normal distribution $p^{(\mathrm{n})}(y)$, which is discussed on page 18 , by changing the variable by $y \equiv \log x$,

$$
p^{(\mathrm{n})}(y) d y=p^{(\mathrm{ln})}(x) d x,
$$

and then by rewriting it by using a new parameter $a$ defined by $\mu \equiv \log a$,

The average of $x$ is not $a\left(=e^{\mu}\right)$, but is in fact $a e^{\frac{\sigma^{2}}{2}}$, and its standard deviation is $a e^{\frac{\sigma^{2}}{2}} \sqrt{e^{\sigma^{2}}-1}$. Further, the median is $a$ and the maximum value of the PDF $p^{(\ln )}(x)$ is at $x=a e^{-\sigma^{2}}$. The plot below is the log-normal PDF $p^{(\ln )}(x)$ for $\sigma=0.1,1,2$ with the average fixed to one.

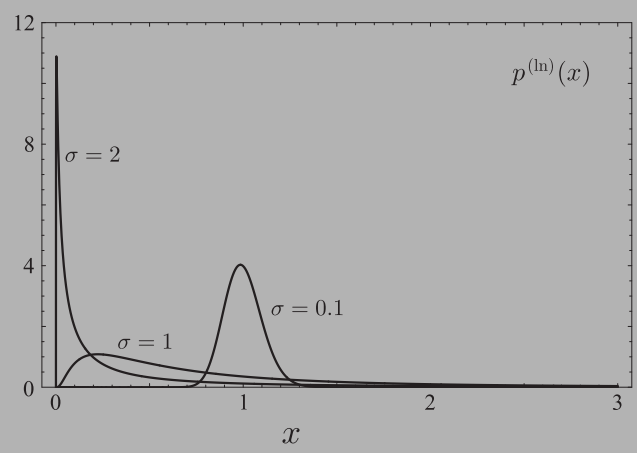

Its $\mathrm{CDF} P_{>}(x)$ is given by the following:

$$
P_{>}(x)=\frac{1}{2}\left[1-\operatorname{erf}\left(\frac{\log (x / a)}{\sqrt{2} \sigma}\right)\right],
$$

where $\operatorname{erf}(x) \equiv(2 / \sqrt{\pi}) \int_{0}^{x} d t \exp \left(-t^{2}\right)$ is the error function. 


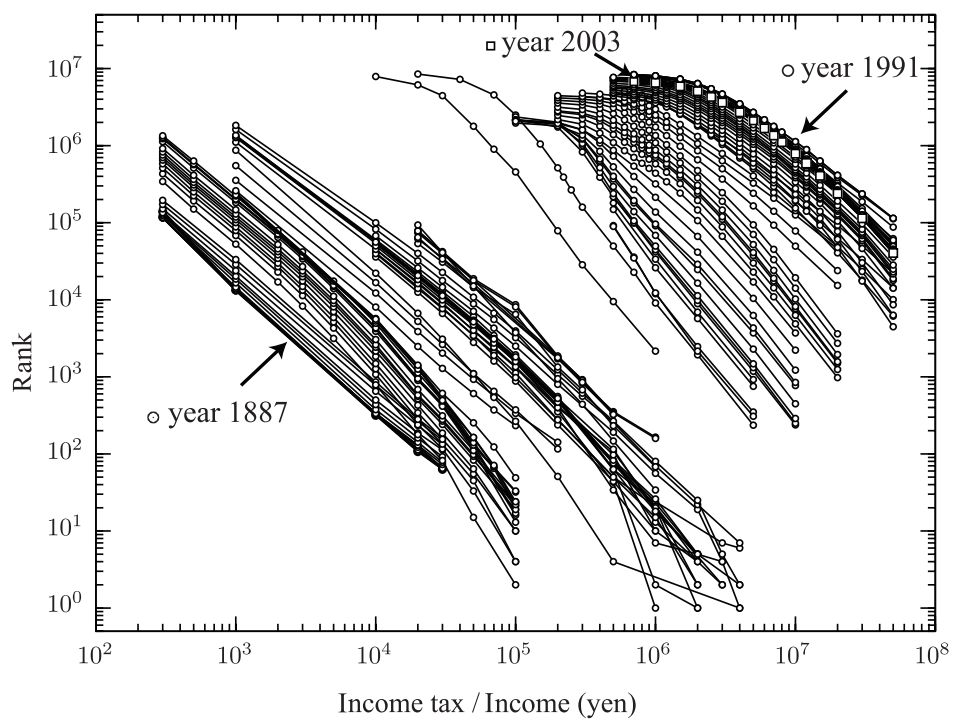

Figure 2.5: Distribution of personal income-tax and income.

into personal perspective.

\subsubsection{Income Distribution and Pareto's Law}

Japanese tax returns have been recorded since 1887, giving a very long data series. However, it should be noted that since the tax system has been changed a number of times, there are some grounds for caution when comparing data from different periods. However, with this caveat in mind, we can look at the rank-size plot of the income distribution from 1887 to 2003 in Figure 2.5, where the abscissa represents the income-tax or income.

The distribution on the leftmost with filled circles is for 1887. After this year, the distribution keeps moving to the right and reaches the rightmost curve with open circles in 1991. After that it reverses its direction and starts to move to the left, reaching the curve in the middle with open squares in 2003. The gap in the middle of the plot divides the earlier distributions of income tax and the later ones of income. Although the data characteristics are different as such, it is at least true that the distributions can be fitted by power-law distributions.

A rough estimate of the Pareto index is given in Figure 2.6, where the abscissa indicates the year and the ordinate the Pareto index. In relation to the inequality of distribution and macroeconomic situation, we observe several things from this plot. 


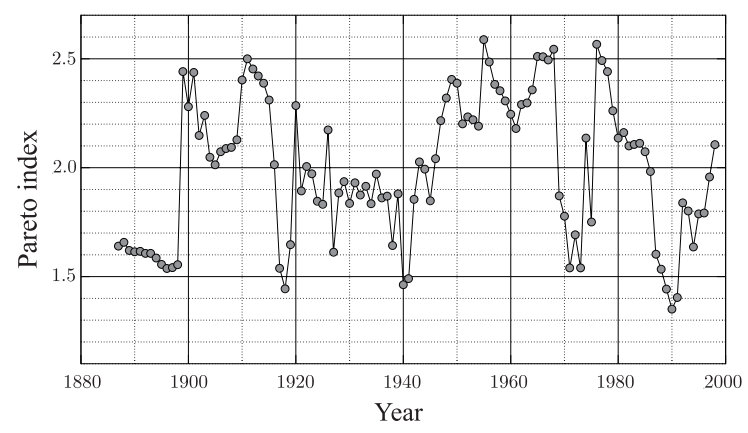

Figure 2.6: Evolution of Pareto index of personal income.

First, the valley-like structure between the 1910's and 1950's. A smaller Pareto index means greater inequality, so a small Pareto index indicates the existence of some people with very large incomes. Therefore, we can see that during this forty years period, the distribution became more unequal and then returned more or less to its earlier state. This phenomenon has been described in the economic literature from a different angle, and it is worth pausing to consider just what happened in this period, and what the economic situation was really like.

In 1955 an economist Simon Kuznets studied the evolution of Gini's coefficient ${ }^{5}$ in the U.S.A., Germany and England, and proposed that in the early stage of economic development inequality in income increases with growth, while countries in an advanced state of economic development the income inequality is reduced and Gini's coefficient becomes smaller, a phenomenon referred to as Kuznets' inverted U-shaped curve hypothesis. ${ }^{6}$ In other words, when economic structure is changing rapidly, the income inequality grows, but reduces again when conditions stabilise. This might be just what happened in Japan from the 1920's to the 1950's.

Another thing we learn from Figure 2.6 is that the income inequality increased in the 1970's. This period was characterised by a real estate boom, encouraged by the then prime minister Kakuei Tanaka, and the oil crisis, both of which may be behind the income phenomena observed.

We can also see in Figure 2.6 that inequality increased again from 1985 to 1998, the so-called "Bubble Economy" of the Heisei era. We will touch again on this point in Section 3.2.2.

Examination of recent income distribution using high-frequency data also yields interesting findings. Japanese personal income in 2000 is plotted in Figure 2.7, where income on the abscissa covers a range from a million to

\footnotetext{
${ }^{5}$ We will elaborate on Gini's coefficient in Section 2.4.1.

${ }^{6}$ Pareto index and Gini's coefficient moved in opposite direction: Larger inequality means smaller Pareto index and larger Gini's coefficient.
} 


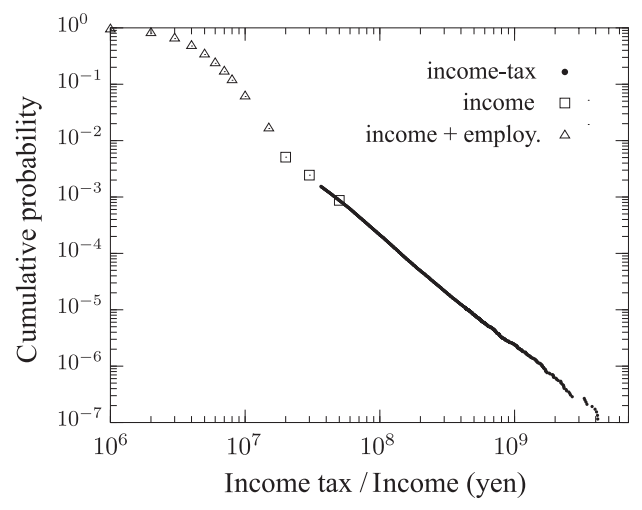

Figure 2.7: CDF of personal income in 2000 (Japan).

several billion yen. ${ }^{7}$ From this plot, we observe that Pareto's law applies to income greater than about twenty million yen. Below this boundary the distribution is different, where we indeed expect the majority of individuals to be on salaries. It is reasonable to expect that these small-to-medium income individuals will exhibit a different distribution to that of the rich, as the latter have income sources other than salary, coming from their investments and other usage of capital.

The relations between the Pareto index, and two risk capitals, the landprice index and average TOPIX (Tokyo Stock Price Index) are shown in Figure 2.8 for 1980 to 1998 .

Note that the land-price index is normalised, with the baseline in 1993, and also that the average TOPIX is the average over the year. The solid line in this plot shows the correlation between the land-price and the Pareto index. We see that from 1980 to 1985 the Pareto index does not change much in spite of the rise in land-price. In the next six years, the land-price increased rapidly with a decreasing index. The land-price peaked in 1991, while the index peaked in 1990, and in 1992, the Pareto index increased significantly, although the land-price did not change a great deal. Afterwards, we see a decrease in land-price associated with a (mostly) increasing index. This kind of motion is registered as a counter-clockwise rotation in this plot.

\footnotetext{
${ }^{7}$ This kind of data is obtained from salary income and declared income published by the National Tax Agency, and income-tax data for high-income individuals published at local tax offices. The former is in a tabulated form, while the latter is a list of all the people who paid income-tax of more than ten million yen. This covers approximately eighty thousand individuals, and thus there are approximately that number of points in this plot. Since anyone with an income of more than twenty million yen must file a tax return, there is an overlapping region between these two kind of data, which allows us to draw one unified profile of distribution.
} 


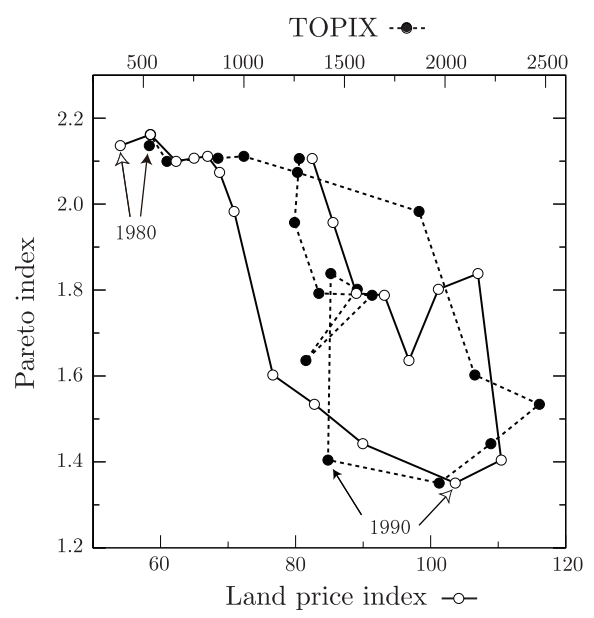

Figure 2.8: Correlation between the Pareto index, land prices, and stock prices (TOPIX).

The broken line in the plot shows a correlation between the averaged TOPIX and the Pareto index. From 1980 to 1984, we see a moderate increase in the average TOPIX, and an almost constant Pareto index. But later, as the average TOPIX increases rapidly, the index decreases rapidly. The average TOPIX peaks in 1987 and starts to decline later, but the index continues to decrease even after 1987, then peaks in 1989 , and starts to decline rapidly as the bubble of the Heisei era bursts.

As we can see from this kind of study of income distribution, the Pareto index fluctuates around 2, with a strong connection to the macroeconomic situation.

In concluding, it should be recognised that this pattern of income distribution, and the evolution of the Pareto index, is not unique to Japan. We have also looked, though not in so much detail, at income distribution in the United States of America and Europe, and similar patterns reveal themselves. Connections between the Pareto index, stock price, and land price are also known to be strong in the United States (Nirei and Souma, 2006). This appears to be a general phenomenon.

\section{Inspiration in Bali and Kolkata}

In the summer of 2002, one of the authors attended an International Conference in Econophysics at Nusa Dua in the island of Bali. The location was a gorgeous resort hotel on a beautiful sun-drenched beach. The days 
were filled with excellent presentations on all kinds of economic and social phenomena, the evenings with culinary delights and cabaret. Very nice, but once outside the resort complex, the reality of Indonesia made itself painfully evident: According to a World Bank report, almost half of the Indonesian population has income of less than two US dollars. And these are dollars which have been adjusted in purchasing power parity, which means that these two dollars indicate money which can buy two dollars worth of goods in the United States. So, this is true poverty.

How can econophysics respond to this situation? One of us was engaged in research relating to high-income people, the results of which are outlined in this section, but he spent a part of his talk in drawing attention to the Indonesian problem, a point which was warmly received by the audience. However the kind of data he needed for a detailed study of poverty was beyond his reach and several of the data resources which he could obtain conflicted with each other.

In 2005, an econophysics conference "ECONOPHYSICS-KOLKATA I" was held in Kolkata, India, and the main theme was income and wealth distribution. Of course, the caste system is still strong in India and there is a huge disparity in income and wealth. One of us got lost in town and wandered around the main and side streets for four hours. The contrasts were memorable; perhaps the most vivid being the sight of a business man in a clean white shirt, talking on a mobile phone, while at his feet an old homeless woman ate rice and vegetables from a newspaper spread on the road.

At the second Kolkata conference, in 2006 the contrasts were if anything sharper. New Suzuki automobiles were a common sight around the hotel, and mobile phones were visible and their ring tones audible all the time, especially in the conference. On the other hand, the town was filled with homeless people, some of whom do not have even a tent.

India is growing nation of extremely energetic people, but when combined with the effects of globalization and other macroeconomic phenomena far from solving the problems these virtually seem to contribute to making the inequalities still larger. Standing there in the streets of India, surrounded by frenetic activity and a heat which is not just atmospheric, we began to wonder whether any government can control the situation and improve matters, and whether econophysics, economics, or any other academic discipline can make any contribution to this seemingly intractable situation. (W.S.)

\subsection{Distribution of Companies}

\subsubsection{Size Distribution of Companies}

In Section 2.1.2, we used the declared income of companies to explain Pareto's law, but other financial data is also potentially relevant in this context, and may actually be more informative. 
(a) Sales (year 2002)

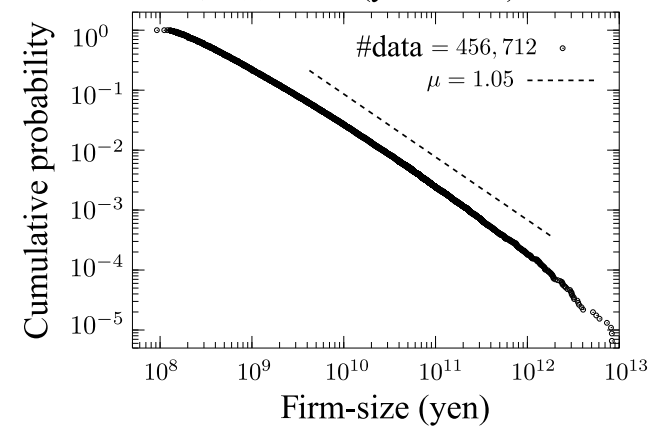

(b) Net profit (year 2002)

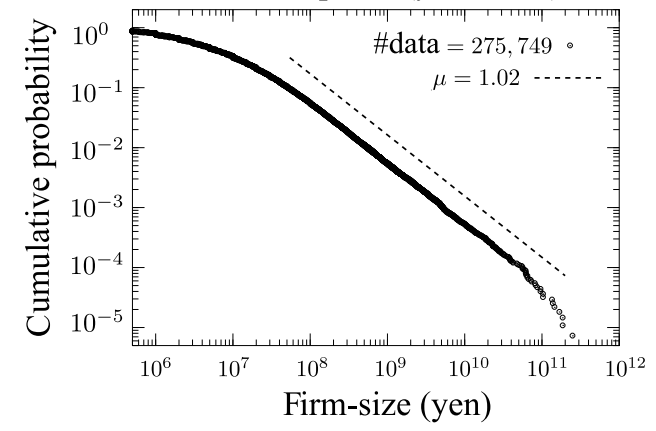

Figure 2.9: CDF of company size: (a) Sales, (b) Income in 2002, Japan.

Figure 2.9(a) and (b) depicts the sales of about 450,000 and the profits of about 270,000 companies, ranging from large to small companies. The potential for data inaccuracy in relation to very small companies should be borne in mind, but information from large companies, those with sales greater than several hundred million yen, is generally regarded as reliable, and the data for profits exhaustive in its detail. In spite of that, it is evident in this plot that large companies obey Pareto's law for these flow quantities. For sales, a rough estimate of the Pareto index in the range from several billion yen $\left(x \simeq 10^{9}\right.$ on the abscissa) to several trillion yen $\left(x \simeq 10^{12}\right)$ yields a value $\mu=1.05$. Similarly for the profit, in the range between several hundred million yen $\left(x \simeq 10^{8}\right)$ and several hundred billion yen $\left(x \simeq 10^{11}\right)$, the Pareto index is about $\mu=1.02$.

Thus, the company size distribution has a Pareto index close to 1 , a very different result from that obtained in regard to personal income.

If we sort the companies into business sectors we find Pareto distributions 
(a) Electrical machinery/supplies

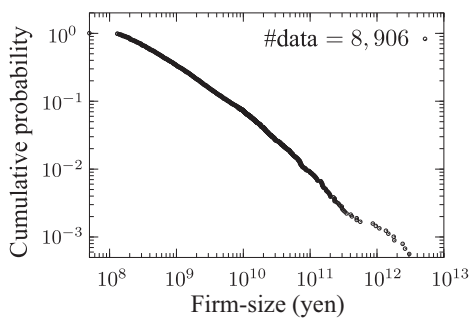

(b) Wholesale

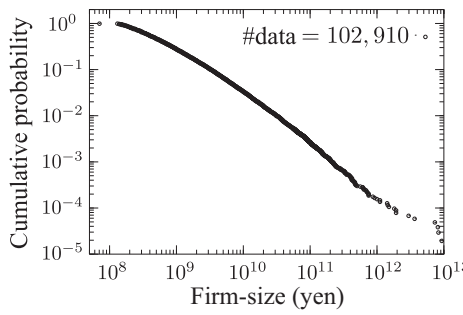

(c) Retail trade

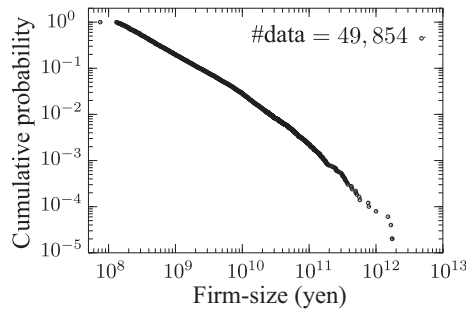

(d) Iron and steel/fabricated metal
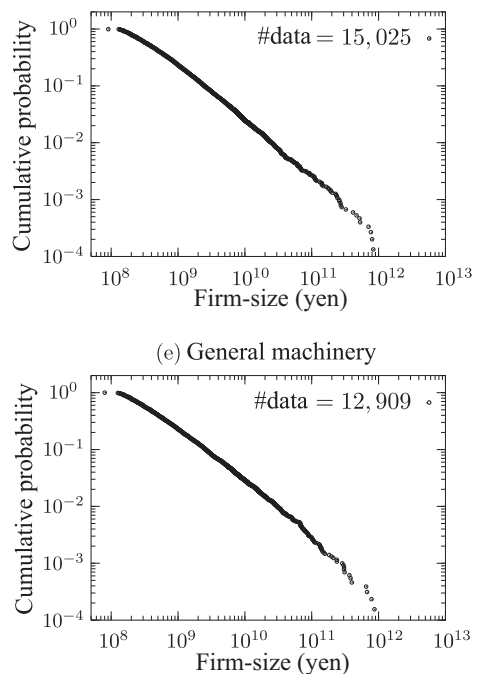

(f) Chemical and petroleum products

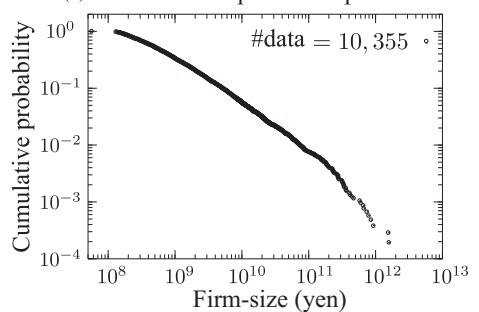

Figure 2.10: CDF of sales in various business sectors in 2002 (Japan).

for most groups. Figure 2.10 shows the CDF for the following categories: (a) Electric and machine making industry, (b) Wholesale, (c) Retail, (d) Steel and other metal-related manufacturers, (e) Machine manufacturers, (f) Chemical and oil-related industry. 
(a) Total-asset (France, 2001)

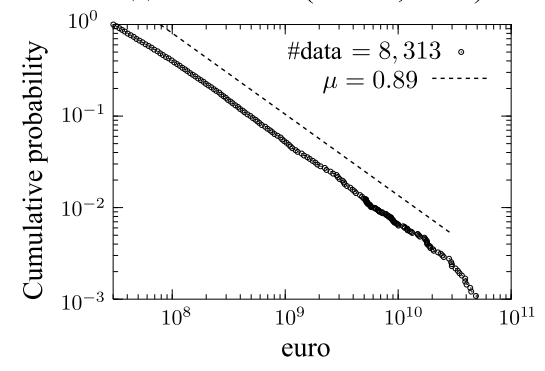

(b) Sales (France, 2001)

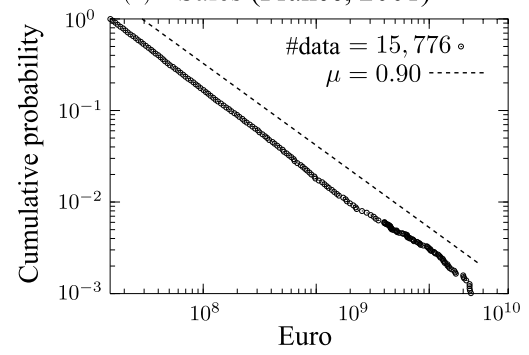

(c) Employees (UK, 2001)

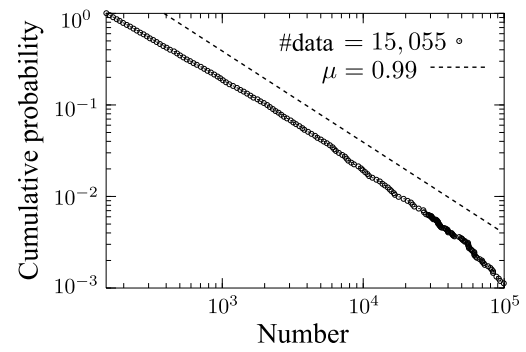

Figure 2.11: The CDF of company size in 2001 for: (a) Total capital in France, (b) Sales in France, (c) Number of employees in U.K..

\subsubsection{Size of European Companies}

As we noted before, company size can be measured either in terms of flows or stocks. The question then arises as to whether the Pareto law holds for both.

Unfortunately, it is not easy to obtain exhaustive data for companies with, say, a total capital greater than a specified value. Fortunately, the European electronic publishing company, Bureau van Dijk, offers a comprehensive database, AMADEUS, which covers all companies with either an operating 
(a) France

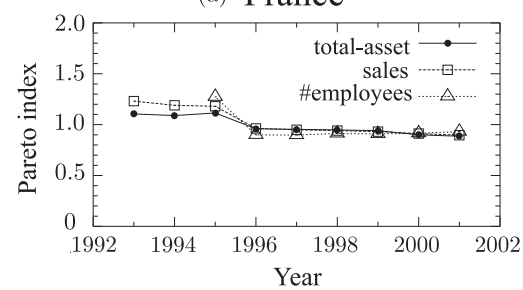

(c) Spain

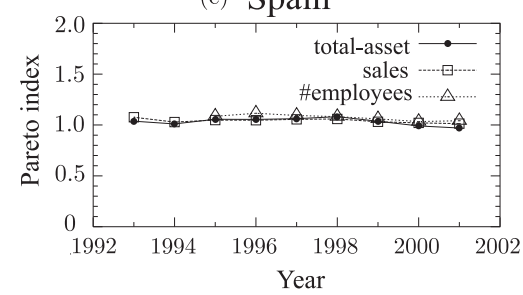

(b) Italy

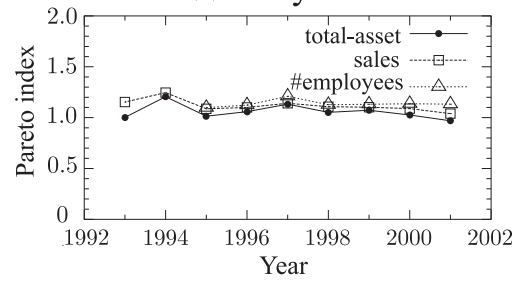

(d) UK

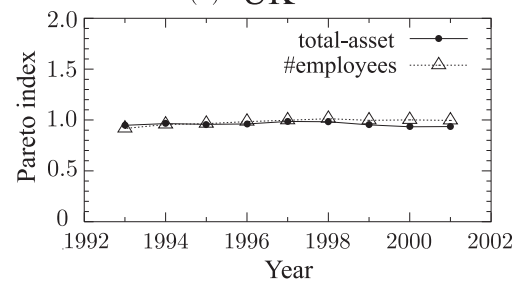

Figure 2.12: Evolution of the Pareto index: (a) France, (b) Italy (c) Spain, (d) U.K., for total capital, sales, and number of employees from 1993 to 2001.

profit greater than 15 million euros, or a total capital greater than 30 million euros, or more than 150 employees. This kind of exhaustive listing is ideal. ${ }^{8}$

Figure 2.11(a), (b) and (c) plot the CDF of Total Capital and Sales of French companies, and the number of employees in British companies. We observe in these plots that no matter which quantity we use for measuring company size, we obtain a Pareto distribution. Rough estimates of the index yield values between $\mu=0.9 \sim 1.0$.

As we will make clear in Section 2.4.3., $\mu=1$ is a boundary, or a critical value where the overall characteristics of the size distribution changes. It should be noted that the value we obtained here for European companies is very close to this value.

In this plot of the evolution of the Pareto index, Figure 2.12, the value is stable, staying very close to $\mu=1$, independently of the quantity used, and the country.

\subsubsection{A Caveat: Sample and True Distributions}

In this section, we will elaborate on a detailed and technical issue. Some readers may want to skip this section and can do so without losing the track of the discussion.

\footnotetext{
${ }^{8}$ The following results were obtained in collaboration with Mauro Gallegati at Ancona,
} Italy. 


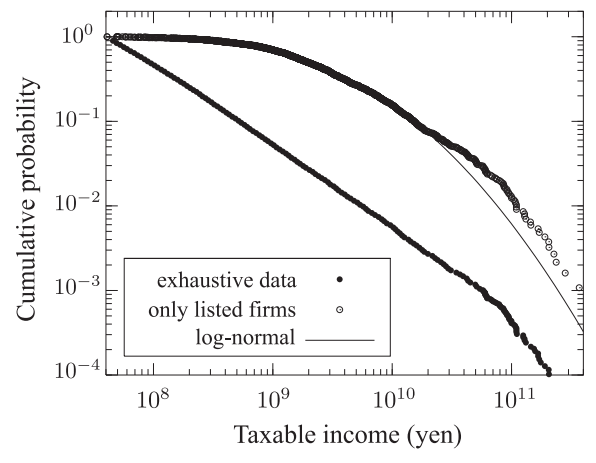

Figure 2.13: gives the CDF for declared company income for all the data (filled circles) and for all listed companies (open circles).

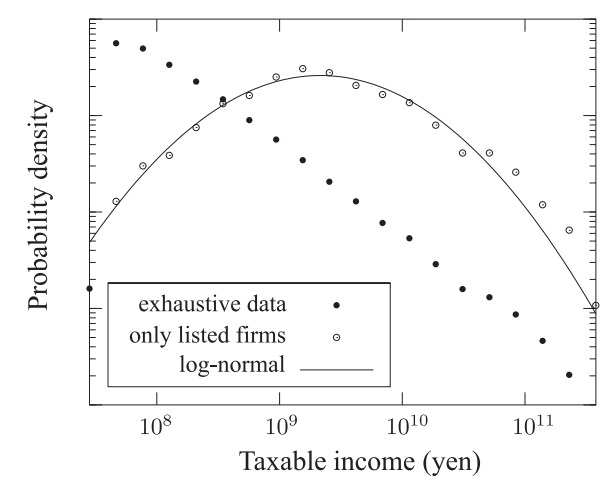

Figure 2.14: PDF of companies' declared income for all the data (filled circles) and data for listed companies (open circles).

Although financial data is accessible for quite a number of companies, our experience shows that they are often limited to listed companies, and it is reasonable to fear that statistical examination which is confined to this set may yield misleading results. Consequently, in order to get a reliable picture of the character of the whole population of companies, not just the listed ones, we need a consistently planned sample investigation, and if possible, data for all or at least a very large part of the population.

Exhaustive data on declared income was used for Figure 2.3. Restricting ourselves to the listed companies might produce a different result.

Figure 2.13 plots the CDF of the subset of all the listed companies (there 
are about two thousand of them) with open circles, while the filled circles represent the $\mathrm{CDF}$ of the entire set of companies. A best fit, with the lognormal distribution, is given by the solid curve. As we noted before, and as is seen clearly in this plot, the log-normal distribution is quite different from the power-law distribution, even though both of them have long tails.

Figure 2.14 shows the same kind of analysis for the PDF. Both of these plots imply that if one had data only for listed companies, one would conclude that the companies obey the log-normal distribution, in spite of the fact that a completely different distribution, Pareto's distribution, applies to the companies in exactly the same region of declared income.

Here we have used data on business tax provided by the National Tax Agency, which made it possible to examine the whole population. But for most financial data such comprehensive coverage is rare. In other less well documented cases we need carefully planned sample investigations of companies, or databases provided by private organisations that specialise in providing information of this kind. Careful preparation of data may lead to reliable statistical conclusions on the population of companies, although sampling of a small number of companies may limit the statistical accuracy.

\subsection{Pareto's law}

We have seen above that a considerable number of financial quantities for both individuals and companies follow Pareto's law, which, in our view, is essential to an understanding of the economic behaviour of these entities. (See also Axtell (2001); Ijiri and Simon (1977); Steindl (1965); Takayasu et al. (1997) among a large body of literature.) Pareto's law also manifests itself in many other economic and social phenomena; indeed, it is ubiquitous. As we have stressed in Chapter 1, if one took for granted elementary statistical concepts such as the normal distribution and applied their implications to all these phenomena, fatal errors would often arise. This is true for other issues involving fluctuations ${ }^{9}$.

In this section, we will elaborate on several important properties of Pareto's law which are necessary for understanding its further implications.

As we explained in Section 2.1.3, the term "Pareto distribution" refers to a group of distributions exhibiting power-law behaviour in the asymptotic region. Therefore, in examining the properties of the Pareto distribution, it is important to distinguish which property is determined by asymptotic behaviour and which property depends on behaviour in the small-to-medium range of the variable. In other words, we need to take care that we select only those properties which are proper to all the Pareto distributions, not just a few of them.

We note that in referring to the scale of the variable $(x)$, it is relative to a proportional constant in the Pareto distribution: The form of its CDF

\footnotetext{
${ }^{9}$ For example, wind power. It is a problem of fluctuation and normal distribution is not obeyed and naive discussion of averaging is groundless.
} 


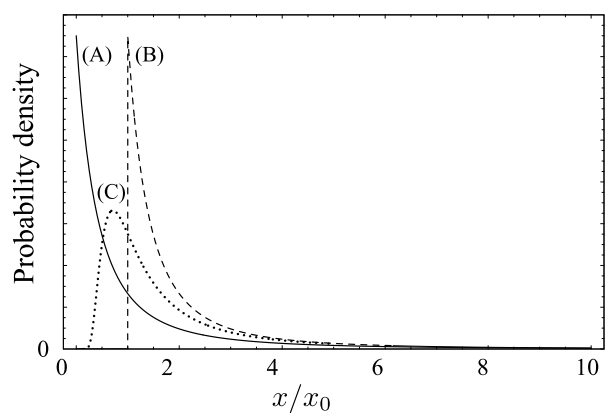

Figure 2.15: PDF of various Pareto distributions.

was given on p.20. Here, we rewrite it as follows by putting its proportional constant in explicit form:

$$
P_{>}(x) \sim\left(\frac{x}{x_{0}}\right)^{-\mu} .
$$

This implies that the asymptotic region is the region where $x$ is much larger than $x_{0}, x \gg x_{0}$, since that is the region where $P_{>}(x)$ is much smaller than 1.

Several Pareto distributions are given in the column below. ${ }^{10}$ The PDFs are given in Figure 2.15, while the CDF's are formed in Figure 2.16. All these exhibit $\mu=1.5$, and show a different behaviour for a finite $x / x_{0}$, but they approach to the same power function as $x / x_{0}$ increases.

When confronted with a distribution a schoolchild would probably want to know the average, the mean. There's no harm in us doing the same: In fact the mean is finite only for $\mu$ larger than 1 and its actual value depends on how the distribution behaves for a finite $x$. When $\mu$ is equal to or smaller than 1 , the average is infinite. Therefore, the way in which the average diverges when it approaches 1 from greater values is common to all the Pareto distributions. It is well-known that the average is anti-proportional to $\mu-1$ :

$$
\langle x\rangle \simeq \frac{x_{0}}{\mu-1} .
$$

As it happens, there are a variety of properties to be examined, and in what follows we first examine some well-known and less-known indices in economics, then we generate some of the distributions with simulations and visualise them as a Devil's Staircase. With that in hand we can then move on to the most important theme, phase transitions, which is in fact our main theme.

\footnotetext{
${ }^{10}$ Kleiber and Kotz (2003) gives several explicit formulae for Pareto distributions, in
} addition to some of other kinds. 


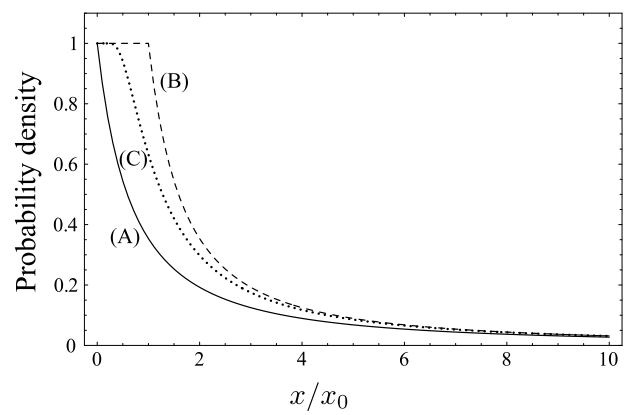

Figure 2.16: PDF of various Pareto distributions.

For the sake of description, we refer to the stochastic variable $x$ that obeys Pareto's law as the company size. Readers may replace it by any other variable.

\section{$\alpha_{\beta}^{\gamma} \quad$ Various Pareto distributions}

Some of the Pareto distributions with a simple function form will be useful for the simulations used later in the book, and we list them here.

As we noted in the main text, the Pareto distribution is defined as a power function in the asymptotic region of its variable $x$. The CDF $P_{>}(x)$ has to satisfy $P_{>}(0)=1$, so that total probability is 1 . But the power-law distribution does not satisfy this property when extended to the finite region of $x$ irrespective of the value of $\mu$. In other words, it cannot be normalised. In order to avoid this problem, we need to modify the behaviour of the CDF for small values of $x$ to obtain a useable probability distribution. (Of course, the CDF is a decreasing function of $x$ by definition, and we must not violate this restriction in such a modification.) Thus, we can obtain an infinite variety of Pareto distributions.

An inverse function of the CDF is needed to conduct a simulation (a point explained in Section 2.4.2 below). Here are three Pareto distributions whose inverse function of the CDF are especially simple.

First, we shift the variable $x$ by $x_{0}$ in the equation on p.36 to obtain the following:

$$
P_{>}(x)=\left(1+\frac{x}{x_{0}}\right)^{-\mu} .
$$

This simple function satisfied all the necessary conditions. We refer to this as Pareto distribution (A) and use it for simulations in this book.

Alternatively, if we suppress the divergence of the power function at $x=0$ we arrive straightforwardly at the following distribution:

$$
P_{>}(x)= \begin{cases}1, & \text { for } x \leq x_{0}, \\ \left(\frac{x}{x_{0}}\right)^{-\mu}, & \text { for } x \geq x_{0} .\end{cases}
$$




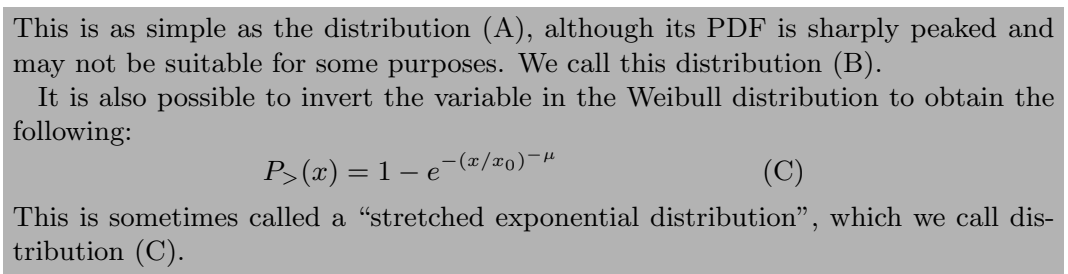

\subsubsection{Gini and Robin Hood}

At this point it seems appropriate to discuss Gini's coefficient and the Robin Hood indices, both of which are measures of inequality. The first of these is well-known, and while the second is a little more obscure it is based on a unique and intriguing idea.

Corrado Gini was an Italian statistician, ${ }^{11}$ who invented a measure for income inequality in 1912, now named after him. The Robin Hood index is another measure invented for the same purpose by Edgar Malone Hoover Jr. (Hoover, 1936). It is sometimes called the Hoover index, and crops up in contemporary literature from time to time, for example in a recent article by Robert Sapolsky (Sapolsky, 2005).

It is interesting to look at these indices in relation to Pareto distributions and the Pareto index.

A Lorenz curve is defined by the following. Let us say that we have $N$ companies, and we arrange them in increasing order, from small to large, and calculate the proportion of the smallest $k$ companies, which we denote by $S_{k}$. Since the total share of all the companies is 1 , we have $S_{N}=1$. Corresponding to the $k$-th (smallest) company, we can place a dot on the coordinate $\left(k / N, S_{k}\right)$. By definition, such dots stay within a square where both sides are equal to 1 . By connecting all these dots, we obtain a line graph that goes up from the lower-left corner to the right-top corner. In actual cases, $N$ is very large and the line graph appears smooth. For this reason, it is called a Lorenz curve. An example is given in Figure 2.17, where the solid line is the Lorenz curve for the Pareto distribution (B) with $\mu=1.5$ in the column on p.37.

If all the companies are of the same size, the Lorenz curve is a diagonal line (the dotted line in the figure), and in general it is below that diagonal line.

Gini's coefficient is defined by the area between the Lorenz curve and the diagonal (the shaded region in Figure 2.17), divided by the area of the triangle made by the diagonal line and the sides, which is $1 / 2$. Therefore, Gini's coefficient is zero if all the companies are of the same size, and is always between zero and 1. The larger Gini's coefficient is the larger the inequality.

Now, Gini's coefficient as defined above depends on the value of $\mu$ as in Figure 2.18 for this distribution. As is seen here, Gini's coefficient increases

${ }^{11}$ His detailed biography is given in Kleiber and Kotz (2003). 


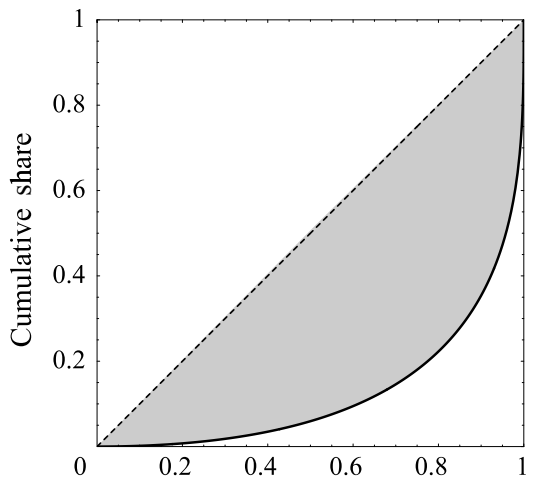

Figure 2.17: An example of a Lorenz curve.

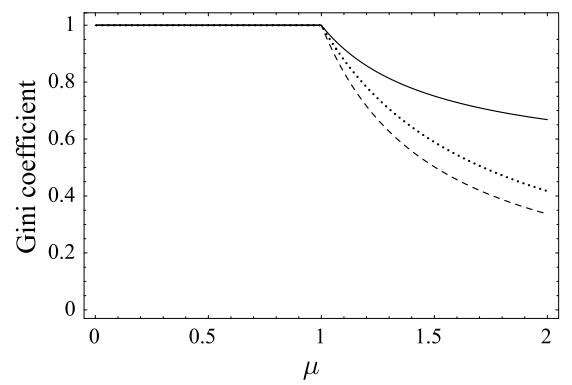

Figure 2.18: The $\mu$-dependence of Gini's coefficient. 


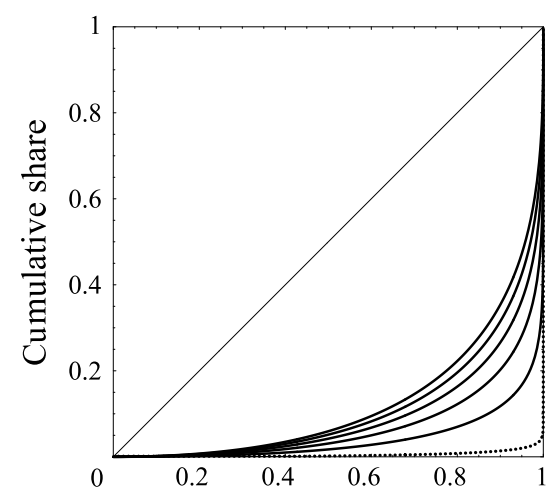

Figure 2.19: Lorenz curves for various values of $\mu$.

as $\mu$ decreases, which is in agreement with the general property of $\mu$, where smaller values of $\mu$ mean the appearance of larger companies and thus higher inequalities.

Once $\mu$ is smaller than 1 , Gini's coefficient is exactly 1 . The Lorenz curve behaves as in Figure 2.19 as $\mu$ decreases, where the solid curves are for the values $\mu=1.5,1.4,1.3,1.2,1.1$ downwards, ending with the dotted curve for $\mu=1.01$ at the bottom. It is evident that as $\mu$ approaches 1 from above, the Lorenz curve shrinks down to the sides, resulting in Gini's coefficient converging to 1 .

The Robin Hood index may be better explained for personal wealth. Let us suppose that we want to create a society where everyone has an equal share of wealth. In order to achieve this goal, we need to move wealth from the rich to the poor in the style of Robin Hood. First we define the boundary between the rich and poor classes. A suitable choice is the person who has the ideal, equal, amount of wealth, a value we can arrive by dividing the total wealth by the number of people. This person is at the place where the Lorenz curve has a gradient equal to 1 , as the gradient is the share of wealth of the person at that point (the black dot in Figure 2.20). This may be more easily understood if one recalls that the Lorenz curve has a constant gradient of 1 when complete inequality is achieved. Those above this person in the rank order are rich, and those below are poor.

The rate of the population of the poor is $R$, as defined in the figure, while the rate of the population of the rich is $1-R$. Therefore, the poor must have a share of wealth equal to $R$ and the rich $1-R$. However, the poor's share of wealth is $S$, while that of the rich is $1-S$. Therefore, our task is to move the share equal to $R-S$ from the rich to the poor. This is equal to the largest vertical distance between the Lorenz curve and the diagonal line, and is shown 


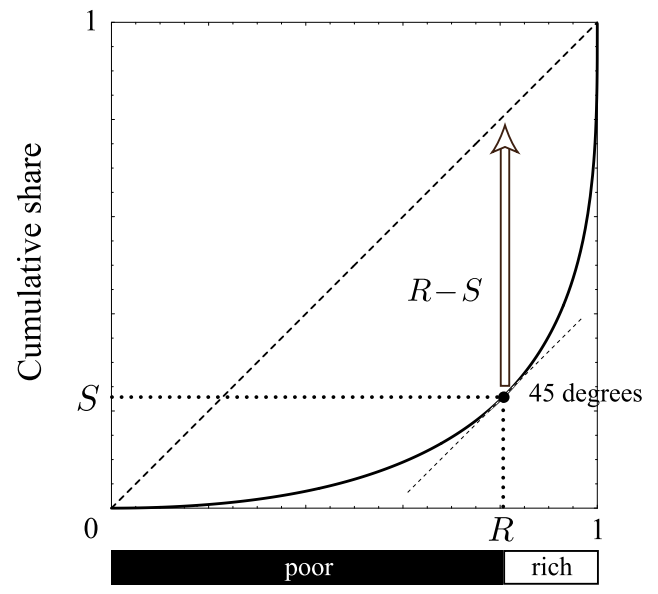

Figure 2.20: Definition of the Robin Hood index.

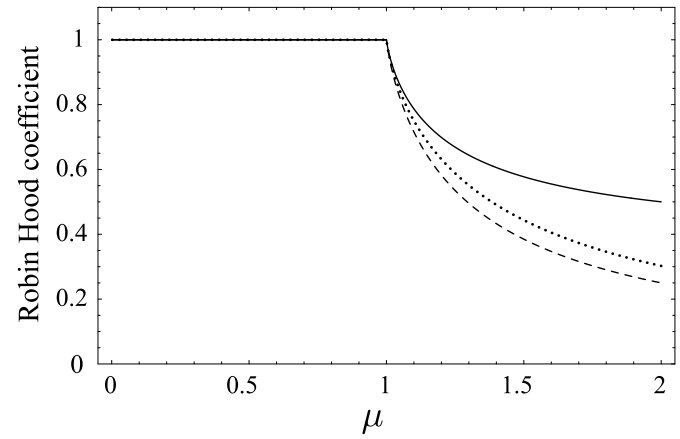

Figure 2.21: $\mu$-dependence of the Robin Hood index.

by the white arrow in the figure. By this definition, this quantity, known as the Robin Hood index, is larger for larger inequalities, with a maximum value equal to 1.

The $\mu$-dependence of the Robin Hood index for the Pareto distributions (A)-(C) is plotted in Figure 2.21. The Robin Hood index is equal to 1 for $\mu \leq 1$, as is evident from the plot of the Lorenz curve Figure 2.19.

The behaviour of the Lorenz curve, Gini's coefficient and the Robin Hood index implies that for $\mu$ equal to or less than one the completely unequal society is realised. Thus the value one of $\mu$ is a very special point. This is 


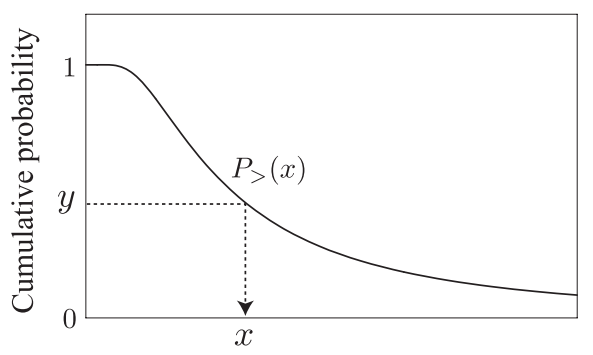

Figure 2.22: The inverse-function method to generate random numbers that obey an arbitrary distribution.

the most important property of Pareto distribution, which we will further investigate.

One note before we proceed: Our previous plots of the Lorenz curve, Gini's coefficient, and the Robin Hood index were appropriate for cases where the number of companies was infinite, when there are a finite number of companies, only when one company holds $100 \%$ of the share, complete inequality is achieved with the Gini's coefficient and the Robin Hood index equal to 1. Analytical simulation methods can be used to estimate the values of Gini's coefficient and the Robin Hood index for a finite number of companies and values smaller than 1 can be obtained.

\subsubsection{Simulation: the inverse-function method}

One useful approach to the properties of the Pareto distribution is to simulate them. For example, we can generate random numbers that obey a Pareto distribution and then calculate various quantities of interest. Large numbers of such simulations yield reliable results, a method called a Monte Carlo simulation, which is perhaps a misnomer since the casinos of that pleasure city seem unlikely to be relying on pure chance.

It is fairly easy to generate a set of numbers that obey a Pareto distribution, and it can be done even with standard spreadsheet applications. Of course, such a calculation is meaningful only when repeated for a sufficient number of times, which may require abundant resources such as a fast PC or workstation, fast and efficient applications, time, and so on. But as the basic idea for generating Pareto distributions is so simple and is applicable to other distributions, it is worthwhile to explain it here. Readers may note that a Monte Carlo calculation with the familiar normal distribution can be conducted with a combination of the method we describe here and a use of a two-dimensional space, which is called the Box-Muller method.

The first step is to generate (pseudo-)random numbers uniformly dis- 


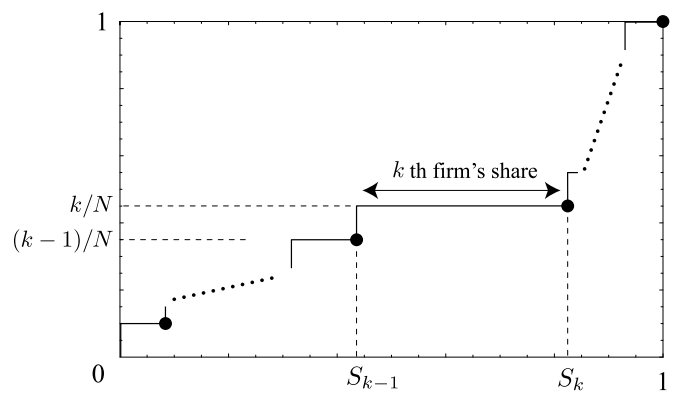

Figure 2.23: How to make a staircase plot. Each dot corresponds to a company.

tributed between zero and one, which we denote by $y$. The necessary function is available in most applications. We then solve the equation $y=P_{>}(x)$ to obtain $x$. Since $P_{>}(x)$ is equal to 1 at $x=0$, it decreases as $x$ increases, and approaches to zero as $x \rightarrow \infty$, the solution $x$ always exists and is unique. By repeating this many times, we obtain a set of solutions for $x$, which obeys the CDF $P_{>}(x)$. This inverse-function method is illustrated in Figure 2.22. The validity of this method is obvious, given the relation between the rank-size plot and CDF explained above.

We have listed several examples of the Pareto distribution on p.37. In the following discussion, we use the distribution (B) to examine the essential properties of the Pareto distribution, since it allows a rapid calculation.

\subsubsection{Devil's Staircase}

One intuitively intelligible method to study the properties of a Pareto distribution is a visualization called a staircase plot.

If we order the companies randomly and plot the total share of the first $k$-companies, as $S_{k}$, then place a dot for the $k$-th company at the coordinate $\left(S_{k}, k / N\right)$, and then connect them with lines, a staircase plot emerges, as illustrated in Figure 2.23. Note that although this is similar to the case of the Lorenz curve, here we have a random order and the vertical and the horizontal axes are exchanged.

In this kind of staircase plot, the flat step of the stair indicates the share of a company, with a wider step indicating a larger share.

Some simple examples will illustrate the method. For example, if there are ten companies and they all have an equal share of $10 \%$, the staircase plot is as shown in Figure 2.24(a). If one company has a 50\% share and the other nine companies share the remainder equally, the staircase plot is as shown in this figure 2.24(b). Of course, a random ordering of the companies creates a 
(a)

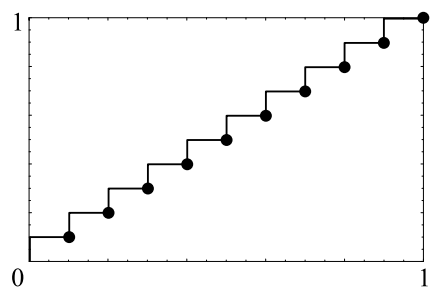

(b)

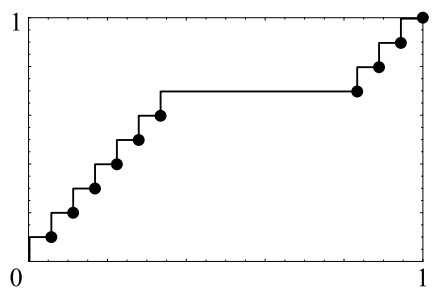

Figure 2.24: Examples of the staircase plot.

staircase plot with the wide step located at a different place. In any case, this kind of staircase plot offers a convenient and accessible visualization of the distribution of the shares.

Once the share is distributed according to a PDF, the stair has irregular steps throughout its extent. Although the height of the steps is equal to $1 / N$ and is constant, the width varies. For a large value of $N$, the staircase becomes very ragged and would be extremely difficult to climb, which is why it has become known as the Devil's Staircase. ${ }^{12}$ Mathematically, it is defined as the mapping of a finite interval between real numbers onto another that is almost universally constant.

Figure 2.25 shows Devil's Staircases for several different values of $\mu$. The data was generated by the method explained in the previous section and contains $N=10^{5}$ companies, but in this figure, we have omitted the dots and just drawn the stairs. We observe that for $\mu=0.8$ (the uppermost two plots) the stair is quite irregular and ragged, and thus is truly devilish. Generating a large number of staircases for a number of different values of $\mu$, we have found that this raggedness is a common property for $\mu<1$ and that the smaller the value of $\mu$, the more ragged the staircase is. On the other hand, for $\mu=1.2$ we observe that the staircase is almost uniform and is like a smooth slope. This is a common property for $\mu>1$ and the larger the value of $\mu$, the smoother the "slope".

Of course it is still a staircase, though the individual steps are not particularly clear since in this case the value of $N$ is large. Closer examination of a selected portion would reveal its detailed features, but we will postpone discussion of this to Section 2.4.6 which covers fractal dimensions.

At the boundary $\mu=1$ an intermediate behaviour is observable, part ragged, part devilish. This extent of this variation depends on the (generated) data.

We have learned from these plots that companies with a large share appear

\footnotetext{
${ }^{12}$ An internet search for "Devil's Staircase" yields several mountain trails, among which is a trail up Mt. Ngauruhoe in New Zealand, which was the location for the filming of Mt. Doom in Peter Jackson's "Lord of The Rings" trilogy, which seems appropriate.
} 

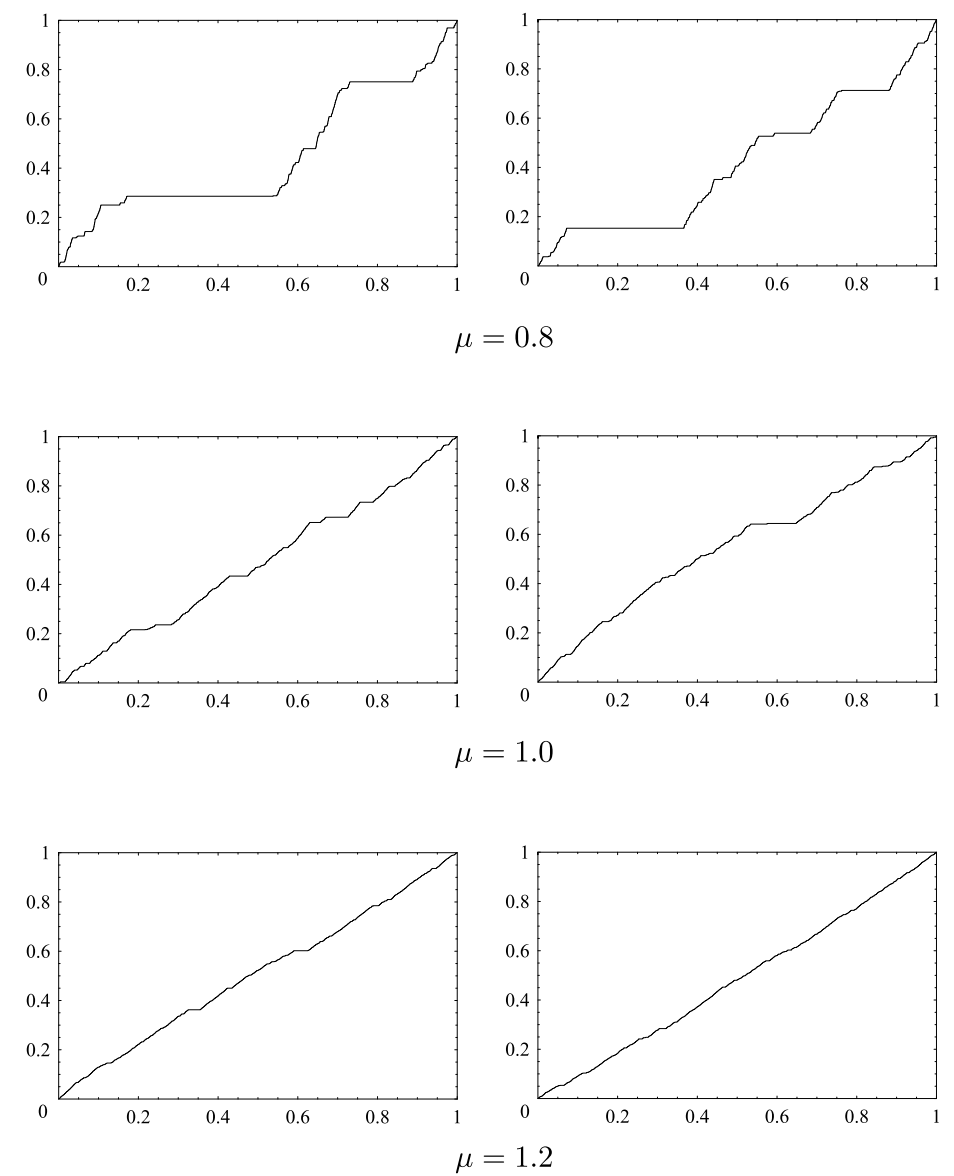

Figure 2.25: Devil's Staircases for $\mu=0.8,1.0,1.2$. 
for $\mu<1$. In fact, in spite of the fact that there are a hundred thousand companies the top two or three companies have nearly half of the share. So, these companies are not just large, but huge. While not quite a monopoly this is obviously an overwhelming oligopoly.

On the other hand, the situation for $\mu>1$ is quite different. Of course, the size of the companies is not uniform and is distributed more evenly, but the staircase is so smooth that it is almost indistinguishable from the case with equal shares. At least, there is no oligopoly.

This argument is based on casual or intuitive observation of the plots, but it can be confirmed by rigorous analysis, which we will see next.

\subsubsection{Oligopoly and Monopoly}

In the previous subsection, we have observed that for $\mu<1$ a Devil's Staircase with exceptionally large companies appears and an oligopolistic situation arises. Here we will look at the share of top companies in order to obtain a quantitative perspective and increase the accuracy of our discussion.

Let us first look at the maximum size. By this we mean the following situation: Suppose we have $N$ companies in total, with sizes $x_{1}, x_{2}, \ldots, x_{N}$.

\section{$\alpha_{\beta} \gamma$ The largest company in a Pareto distribution}

When there are $N$-companies, the size of the largest company is given by the following when $\mu$ is larger than 1 :

$$
\left\langle X^{(\max )}\right\rangle=\Gamma\left(1-\frac{1}{\mu}\right) N^{1 / \mu}
$$

This is common to all the Pareto distributions. Here $\Gamma(z)$ is the Gamma function obtained by a suitable analytic continuation of the following expression:

$$
\Gamma(z) \equiv \int_{0}^{\infty} t^{z-1} e^{-t} d t
$$

At $\mu=1$ and when $N$ is large, its share is

$$
\frac{\ln (\ln N)-\gamma_{E}}{\log N}
$$

where $\gamma_{E}=2.7799 \ldots$ is the Euler's constant. The numerator of this expression varies very slowly with $N$ and thus we have elsewhere described it as "almost antiproportional to logarithm of $N "$.

For a value of $\mu$ less than 1 , when $N$ is infinite, the share of the finite-rank company is known analytically. Since it is a complex expression including an incomplete gamma function, we will not go into the details here, but, we note that it is somewhat surprizing that while a Pareto distribution is defined by such a simple power-law behaviour it also yields complex expressions for these quantities through a long series of analytic calculations. This is the joy of Mathematics. 


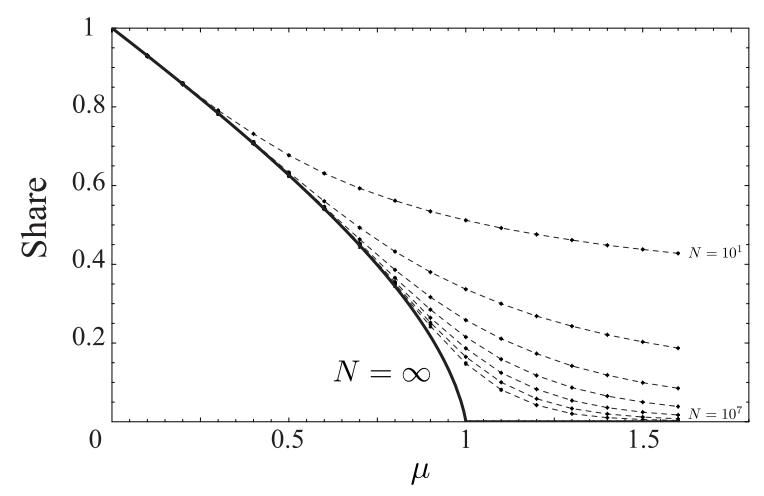

Figure 2.26: Share of the largest company.

We can measure and anticipate the maximum of value of these sizes. In Pareto distributions with a large $N$ and an $\mu$ larger than 1, the average maximum size is proportional to $N^{1 / \mu}$. This is common to all the Pareto distributions. As stated above, the average size does not have this property. A difference arising from the fact that the maximum size is determined by the behaviour of the distribution at large values of $x$, when $N$ is also large. And this is stronger as $N$ increases. Therefore, the dominant term in the expression of the average of the maximum size for large values of $N$ is determined by the asymptotic behaviour of the distribution, which is common to all the Pareto distributions. We will give an explicit formula of the proportional constant in the column on p.46, but here we only note that it diverges as $\mu$ approaches to 1 from above, in agreement with the fact that the average size diverges at the same time.

Next, we will look at the share of the top companies, which is crucial in determining the overall appearance of the Devil's Staircase.

Figure 2.26 indicates the results of the Monte-Carlo calculation and the analytic calculation for the average of the share of the top company. The thick, solid, curve denoted with $N=\infty$ is the analytic result for an infinite number of companies $(N=\infty)$, while the lines above it show the results of the Monte-Carlo calculation for ten, one hundred, ..., and ten million $\left(N=10^{7}\right)$ companies from top to bottom. As $N$ increases the results of the simulation converge on the analytic results for $N=\infty$, as they should. (In this simulation, one hundred thousand data points were generated by the Monte Carlo method for most $N$, except that for $N=10^{7}$ one thousand data were generated. This is sufficient to guarantee the statistical accuracy of the result.)

As can be clearly seen, the average share of the top company is zero for $\mu$ greater than 1 for $N=\infty$. One might think this is a trivial result: Since 
there are an infinite number of companies, the share of any company must be zero, but this is a misleading argument.

If one is dealing with distributions that do not have long tails, such as the normal distribution, the above argument is sufficient, but it isn't the case for Pareto distributions, which have long-tails.

We need to look carefully at what is really happening for Pareto distributions. As we have noted already, the average size is finite for $\mu>1$. Therefore, the average of the sum of the size of all $N$ companies is proportional to $N$. On the other hand, the average of the maximum size is proportional to $N^{1 / \mu}$. The simple ratio of these yields

$$
\frac{N^{1 / \mu}}{N}=N^{(1 / \mu)-1}
$$

which offers an estimate of the average share of the top company. So we find that the exponent of $N$ is $(1 / \mu)-1$, which is negative for $\mu>1$. Therefore, this ratio goes to zero as $N$ goes to infinity. The behaviour of the average share of the top company in Figure 2.26 for $\mu>1$ agrees with this estimate.

When $\mu$ is smaller than 1 a completely different situation arises. In this case, both the average share and the average of the maximum size diverges when $N$ goes to infinity. Therefore, the simple estimate we made above is not valid, ${ }^{13}$ so we need a different calculation for this case. Since it is long, tedious and quite complicated, we will not give the derivation, but simply give the resulting plot in Figure 2.26 with a thick solid curve.

We have seen in the Devil's Staircases that even though there are a large number of companies the top few had major shares. Correspondingly, we see that even when the number of companies is infinite, the top company has a finite share. As we noted when dealing with $\mu>1$, this also shows how dangerous it is to apply our naive "intuition", which has been raised on normal distributions, .

To repeat, as soon as $\mu$ crosses the value 1 and becomes less than 1 , the average share of the top company is finite. In spite of the fact that there are an infinite number of companies, the top company has a finite share. As $\mu$ approaches to zero, that share approaches to 1 , in other words, a complete monopoly.

This is, of course, caused by the long tail of the Pareto distributions. The smaller $\mu$ is, the longer the tail, and on that long tail we find exceptionally large companies. As soon as $\mu$ becomes smaller than 1 , the quantitative nature of the size of those extremely large companies changes drastically.

The average share of the second-largest company behaves as in Figure 2.27, where results similar to the top company are observed. The average share is finite for $\mu$ less than 1 in spite of the fact that there are an infinite number of companies.

\footnotetext{
${ }^{13}$ This is also understandable, as the above estimate yields infinity for values of $\mu$ less than 1 , and $N$ also goes to infinity, which is simply wrong because the share must be less than 1 by definition.
} 


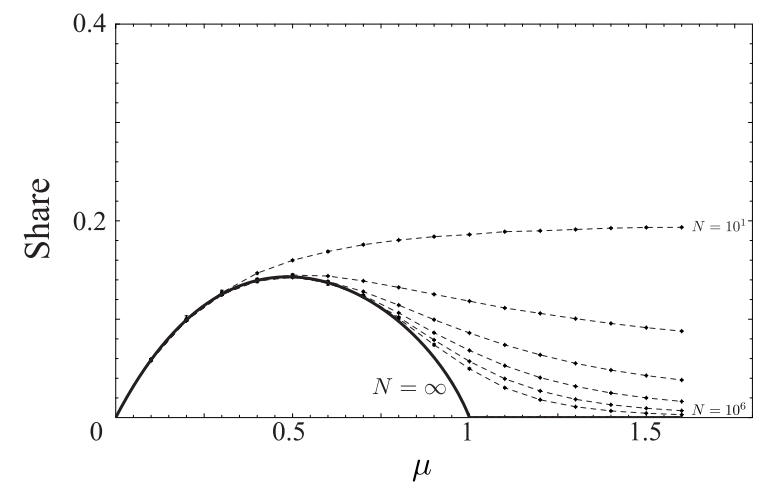

Figure 2.27: The average share of the second-largest company.

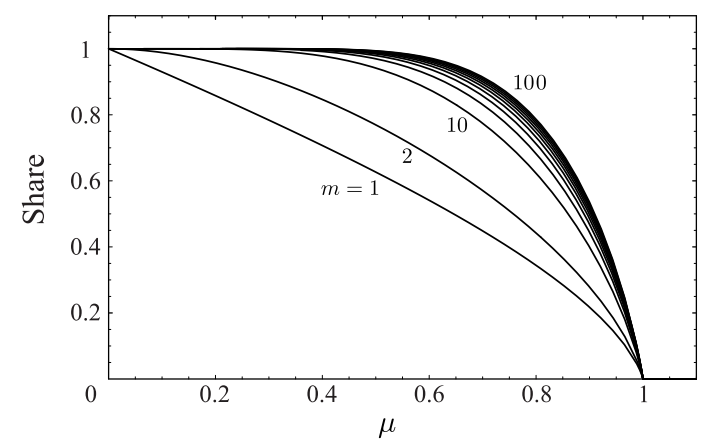

Figure 2.28: Total shares of the top companies.

We have also obtained the shares of the top one hundred companies for $N=\infty$ and plotted their sum in Figure 2.28. The lowest curve denoted by " $m=1$ " is the share of the top company, the curve above it denoted by "2" is the sum of the shares of the top and the second companies, and so forth, ending with the sum of the share of the top one hundred companies denoted by " $100 "$ ".

Some of the representative values of the shares of the top companies are listed in Table 2.1, where it is evident that even when $\mu$ is less than 1 by a small amount, the oligopoly is severe.

The sum of the shares of the top to the $\infty$-th company add up to 1 for $\mu<1$. One might think this is a trivial fact, but it is not that simple, since in calculating the shares we took the total number of the companies, $N$, to 
Table 2.1: Average shares (\%) of the top ten, fifty, and one hundred companies for values of $\mu$ closer to 1 .

\begin{tabular}{|c|c|c|c|}
\hline$\mu$ & Top 10 & Top 50 & Top 100 \\
\hline \hline 0.90 & 40.0 & 49.8 & 53.5 \\
\hline 0.95 & 24.2 & 30.5 & 33.0 \\
\hline 0.98 & 11.8 & 14.7 & 15.9 \\
\hline
\end{tabular}

be infinite. If we defined $N$ as a finite number and added the shares from the top to the $N$-th company, it should add up to 1 . If we deined $N$ as infinite, distributions without long tails, like the familiar normal distribution, would yield zero since the share of any company of a finite rank is zero and zeros add up to zero. This happens for Pareto distributions with values of $\mu$ greater than 1. Therefore, it is somewhat surprising, or one might say, spectacular that they add up to 1 for values of $\mu$ less than 1 .

We can also look at the distribution of the shares, not just their averages. Figure 2.29 illustrates the PDF of the share of the top company for $\mu=$ $0.2,0.4, \ldots, 1.4$ and $N=10,10^{2}, \ldots 10^{7}$. In all the plots, the curve closest to " 10 " is for that case, and $N$ becomes smaller as the curve moves further away. Also, for any value of $\mu$, the average share becomes smaller as $N$ becomes larger. So, it should be easy to use this fact to determine which curve is for which $N$. From these plots, we see that if $\mu$ is larger than 1, the distribution is concentrated around the value of the share equal to zero, and as $N$ becomes larger the concentration becomes more intense. This is in agreement with the fact that the average is zero for an infinite $N$. On the contrary, if $\mu$ is less than 1 , the distribution is spread over the whole region from zero to 1 . This shows that in addition to the fact that the average is of order 1, the share of the top company for each data set could be quite different from the average. That is, the order of fluctuation of the share of the top company is of order 1.

\subsubsection{Pareto's 80-20 rule}

It is important to distinguish the so-called "Pareto principle", or "Pareto's 80-20 rule", from "Pareto's law". Some posited instances of the 80-20 rule are listed below.

- $80 \%$ of sales are to $20 \%$ of consumers

- $20 \%$ of time used for a study results in $80 \%$ of the conclusions

- $80 \%$ of crime is committed by $20 \%$ of population

- $80 \%$ of happiness in life is derived from $20 \%$ of time

- $80 \%$ of marriages involve that $20 \%$ of the population with divorce histories 

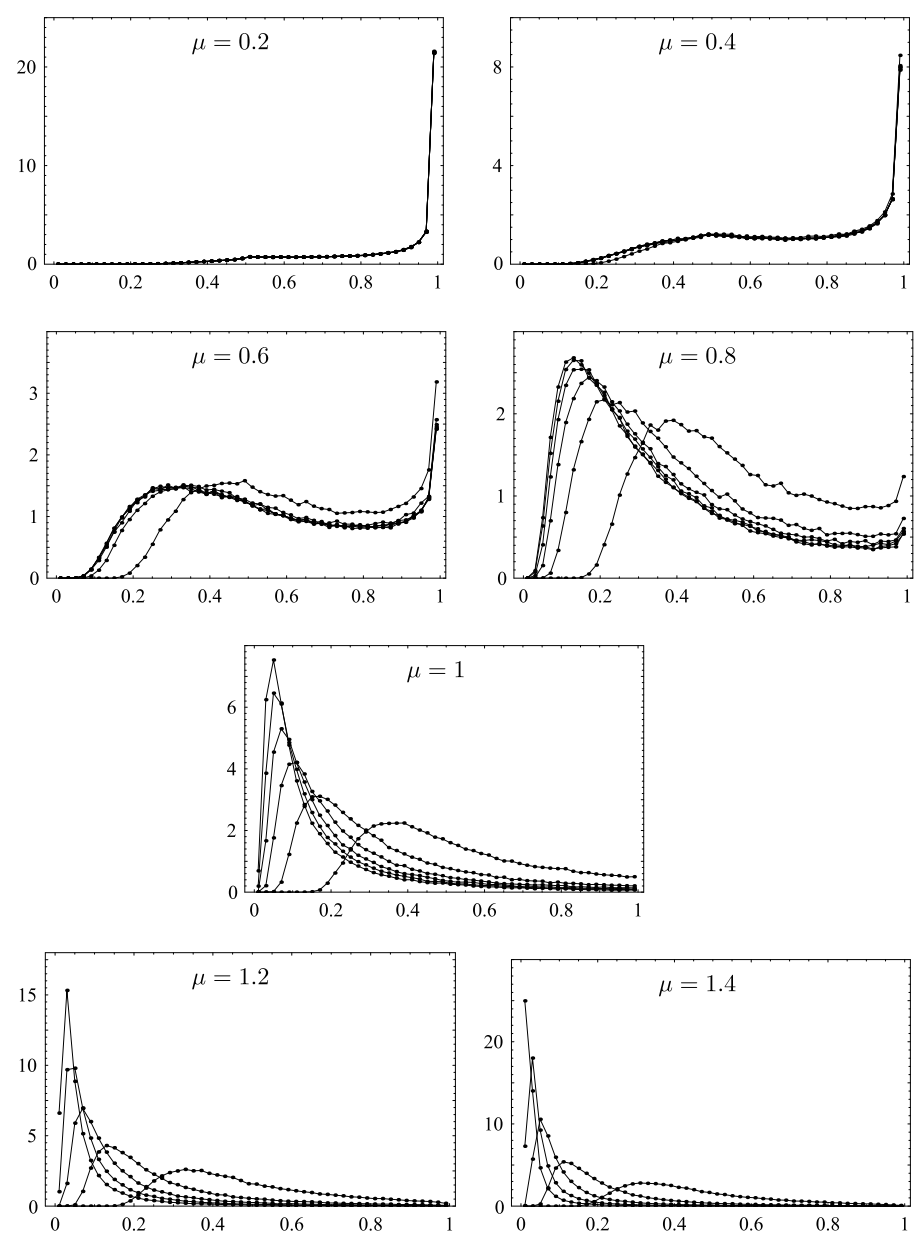

Figure 2.29: Distribution of the share of the top company. 


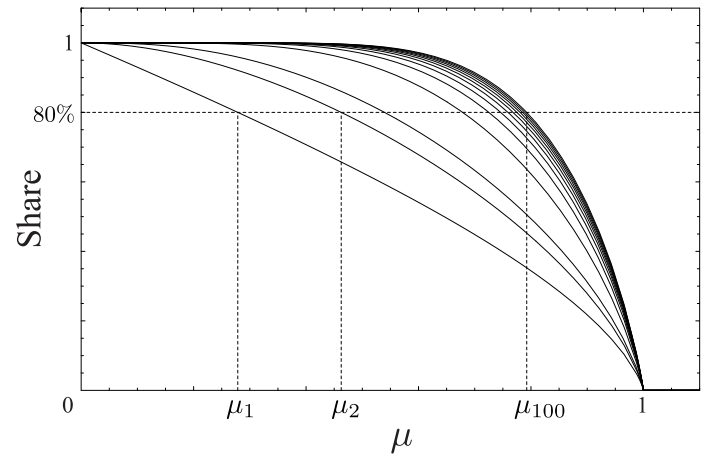

Figure 2.30: The value of $\mu$ below which the top $n$-companies achieve a total share greater than $80 \%$.

and so on and so forth. Some of these are obviously dubious. Sometimes, they are referred to as a solid and proven universal scientific law, and the power-law is referred as a justifying foundation. This is a rather dangerous trend, bordering on "pseudo-science". By looking at this rule by means of the properties of the Pareto distribution we may be able to shed a more scientific light on it.

For $\mu<1$, it is easy to see what really happens: Even if $N$ is infinite, the top companies have finite shares. Therefore, if we take the top $20 \%$ of companies, there are an infinite number of them and their total share is $100 \%$. The "Pareto principle" appears to be wrong.

In Figure 2.28 we have drawn a line for the share equal to $80 \%$ and plotted the rank of those achieving that share in Figure 2.30. We readily see that for $\mu<\mu_{1} \simeq 0.28$ the top company has a share greater than $80 \%$, while for $\mu_{1}<\mu<\mu_{2} \simeq 0.46$ the top and the second company have a total share more than $80 \%$, and so on. Further, for $\mu<\mu_{100} \simeq 0.79$, the top one hundred companies have a total share greater than $80 \%$. We have plotted the rank that achieves the $80 \%$ share in Figure 2.31, from which one can read the value of $\mu$ under which the top twenty companies achieve a $20 \%$ share.

On the other hand, if $\mu$ is greater than 1 , the share of the top $20 \%$ of companies is less than 1. But one should be careful in dealing with actual numbers, since this share is not common to all the Pareto distributions, but rather depends on its behaviour in the non-asymptotic region, where its stochastic variable $(x)$ is not large. This returns us to a point of caution raised earlier, namely that the "Pareto distribution" refers only to its behaviour. To put this another way, it is the name of a class of distributions that approach to the power function as the variable $x$ goes to infinity. Each has a different behaviour for small-to-medium values of $x$. What we have studied above, such 


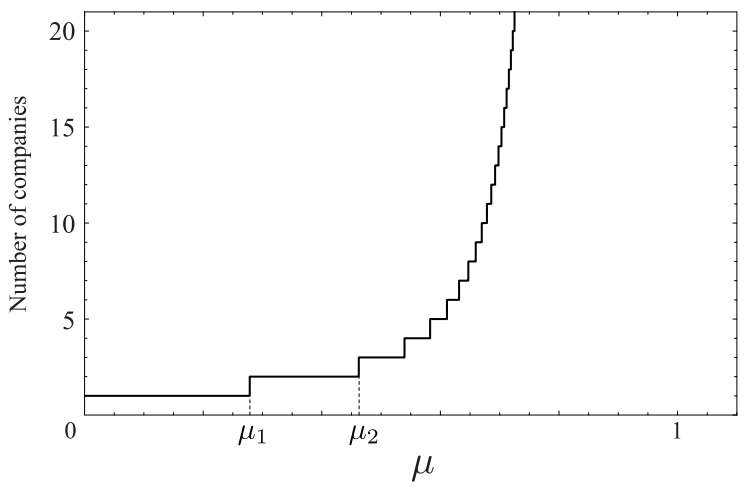

Figure 2.31: The minimum number, $n$, of the top companies, whose total share is greater than $80 \%$.

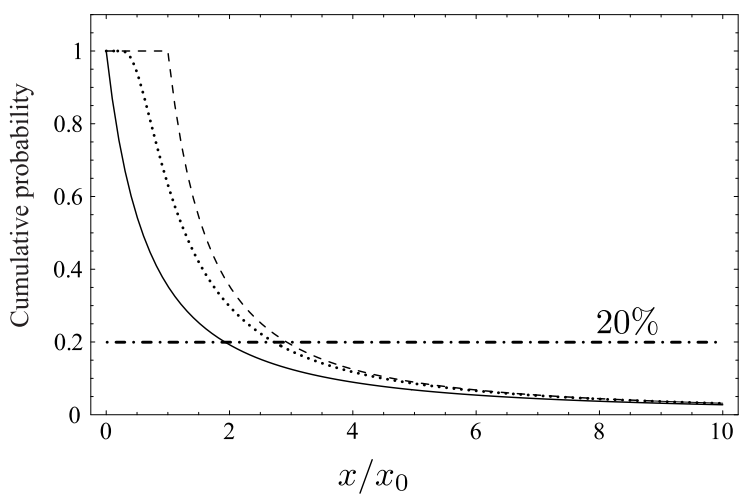

Figure 2.32: Various Pareto distributions and the $20 \%$ line.

as the behaviour of the largest company, depends only on the power aspect for large values of $N$, and is common to all the Pareto distributions with the same value of $\mu$. But we are now thinking of the top $20 \%$ of companies, and their properties are uncommon, no matter how large the total number of companies, $N$. This can be most easily understood by looking at Figure 2.32. The top $20 \%$ of companies are below the horizontal line denoted by "20\%". The distribution of their size, $x$, is given by the curve of distribution, which differs from one distribution to another. Therefore, it is not sufficient to speak casually about using a Pareto distribution; we have to specify which Pareto distribution we are using. 


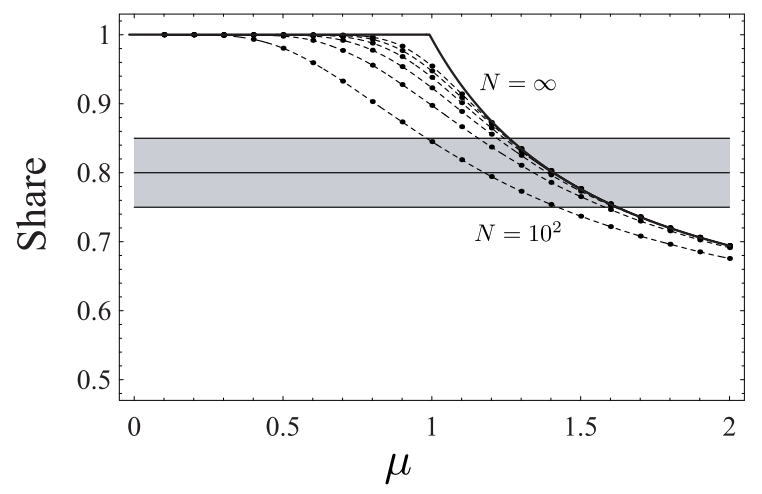

Figure 2.33: Dependence of the share of the top 20\% companies on $\mu$.

With this caution in mind, we can use our Monte Carlo method to investigate the Pareto distribution (A) given on p.37. The result is illustrated in Figure 2.33, where the dots connected by broken lines are the results of a Monte Carlo calculation with $N=10^{2}, 10^{3} \ldots 10^{8}$ from below, and the thick curve is for $N=\infty$. As we stated above, for values of $\mu$ less than 1 a finite number of top companies have the $100 \%$ share and therefore the thick curve is exactly 1 for $\mu \leq 1$. The grey area is where the share is between $75 \%$ and $85 \%$, so, one might say that when the desired broken line or the thick curve is within this grey area, the top $20 \%$ companies have about $80 \%$ of the share. Also, Table 2.4.5 lists the values of $\mu$ where the top $20 \%$ companies have the share $75 \%, 80 \%$, and $85 \%$. From this we see, for example, that when there are ten thousand companies the top $20 \%$ of companies have about $80 \%$ of the share for $\mu$ between 1.2 and 1.6. In Figure 2.34, we plotted the value of $\mu$ where the top $20 \%$ of companies have the share $75 \%, 80 \%$, and $85 \%$ with three curves. Further in this plot, we gave the range where we did not restrict the rate of the companies to exactly $20 \%$ but extended it to range between $15 \%$ and $25 \%$, as denoted by the grey area. From this, as soon as there are a certain number of companies, say one thousand, for values of $\mu$ a little greater than 1 and less than 2, the 80-20 rule holds approximately. Of course, this is the result for the Pareto distribution (A), but it is easy to see that a similar result will hold approximately for other Pareto distributions.

As we have seen here, the 80-20 rule is not unconditionally guaranteed by the power law, but it is approximately valid in some regions of $\mu$ (and $N)$. Since in practice quite a variety of Pareto distributions have a value of $\mu$ somewhere between 1 and 3 , the $80-20$ rule is valid to some extent, which accounts for its popularity.

Even so, we should be aware that the 80-20 rule is not justified in any precise sense. What is important in practice is to see which kind of distributions 


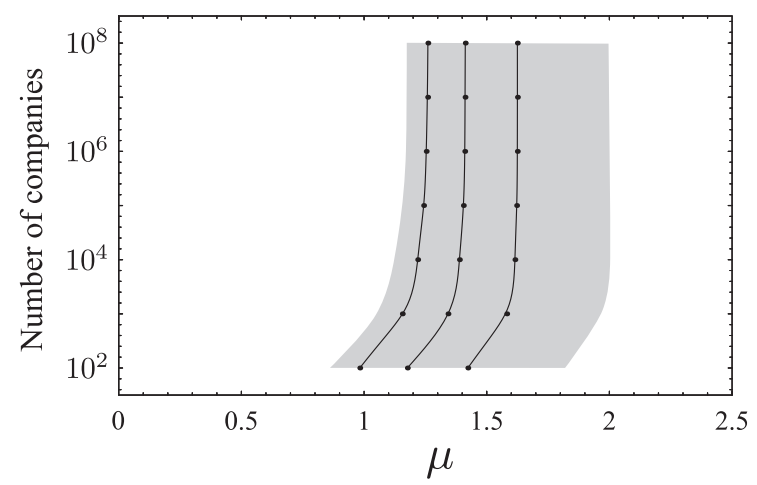

Figure 2.34: The range of $m$ where the $80-20$ rule holds.

Table 2.2: The value of $\mu$ below which the top $20 \%$ of companies have a combined share of $75 \%, 80 \%$, and $85 \%$.

\begin{tabular}{|c|c|c|c|}
\hline$N$ & $75 \%$ & $80 \%$ & $85 \%$ \\
\hline \hline $10^{2}$ & 1.42 & 1.18 & 0.98 \\
\hline $10^{3}$ & 1.58 & 1.34 & 1.16 \\
\hline $10^{4}$ & 1.61 & 1.39 & 1.22 \\
\hline $10^{5}$ & 1.62 & 1.40 & 1.24 \\
\hline $10^{6}$ & 1.62 & 1.41 & 1.25 \\
\hline $10^{7}$ & 1.63 & 1.41 & 1.26 \\
\hline
\end{tabular}

the quantity in question obeys, and in what range, and to carry out a correct statistical analysis, including the estimate of possible range of errors. One may end up with a Pareto distribution, but, equally, the result might well be quite a different kind of distribution. Robust business or governmental strategies can come only from this kind of examination. In other words, "Pareto's 80-20 rule" belongs only in casual conversation, and could cause real problems if it is allowed to escape into analysis with serious consequences.

\subsubsection{The Fractal Dimension}

The "Fractal" is a mathematical concept discovered by a French-American mathematician, Benoit Mandelbrot. Since his famous book Fractals: Form, chance, and dimension was published in 1977 the number of scientific papers developing the concept has kept increasing, and now covers a wide range of disciplines. Indeed, fractals crop everywhere in science and technology. There are even industrial applications such as fibres with a strongly water-repellent fractal surface, and fractal antennae that are smaller and more powerful than 
conventional ones.

It is probably less well-known that the concept of the Fractal can also be used in the study of complicated behaviours in a market, for example movements in cotton prices, exchange rates, and stock values. None of these values obey ordinary, smooth, functions, but jump about all over the place. In other words, they are ragged, ${ }^{14}$ and such raggedness is apparent even if we focus on the detail, a property referred to as self-similarity. In fact, since real market data has a minimum time-interval the similarity fades as we close in on that minimum unit of time-scale. Similar structures are commonly seen in nature, a famous and well-studied example being coastlines.

Fractal objects are thus characterised by raggedness and self-similarity, which is measured by their fractal dimension. Think of it like this. The ordinary smooth lines we draw on paper by hand have a dimension of 1 . If we draw a circle and fill it in with black ink, we obtain a two dimensional object. A simple dot has a zero dimension. Turning to the ragged lines of exchange rates, stock prices, and coast lines we might assume that they are one-dimensional since they form a line. But this is not necessarily true. In fact, the length is uniquely determined for ordinary lines, and we can measure it by using a divider. We split the line into segments of the unit length of the divider, and by multiplying the number of segments with the unit length we obtain an approximate value for the total length. By reducing the unit length of the divider, we can get a more accurate value. If it approaches to a unique value as we reduce the unit length, we define that value as the length of the line.

However, a ragged line may not have that limiting value. Often the measured length becomes longer as the unit length becomes smaller. In such a case, it cannot be regarded as an ordinary one-dimensional line. On the other hand, since the ragged line does not cover an area of a region, it is not a two-dimensional object either. The dimension of such a line is somewhere between 1 and 2 and is not an integer; it has a fractal dimension. This is basically the condition of calling an object fractal.

In the previous subsection, we have visualised the shares of companies $\left\{S_{k}\right\}$ using the Devil's Staircase. This is one example of a fractal.

We will now count the fractal dimension $D$, whose definition is illustrated in Figure 2.35. We divide the region $(0,1)$ of the abscissa into small segments of equal size, some of which contain dots and some of which are empty. We denote the number of non-empty segments by $k(n)$ when there are $n$ segments, If the dots cover the region $(0,1)$ then $k(n)$ should be equal to $n$. For example, consider the set of all real numbers in the region $(0,1)$, which is just a line segment of length 1 and is one-dimensional. (Alternatively, we could select the set of all rational numbers or the set of all nonrational numbers.) In this case, since there are real numbers in any segment of non-zero length, $k(n)=n$. On the other hand, if the set is made with only a point in $(0,1)$, its dimension is zero and $k(n)$ is always equal to 1 independently of $n$. From this, we assume

\footnotetext{
${ }^{14}$ In other words they are "not universally differentiable".
} 


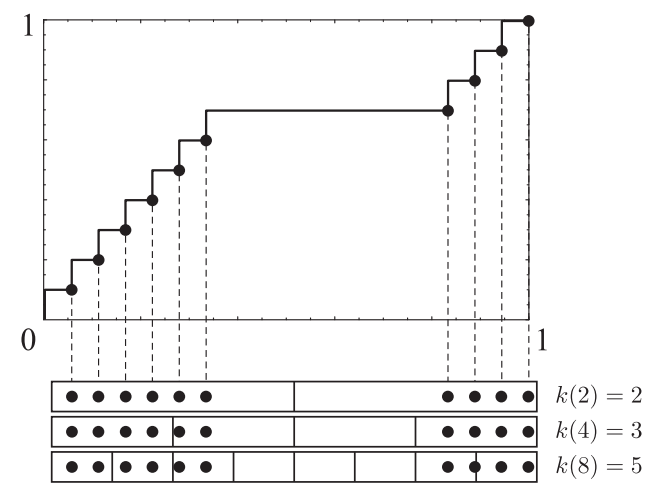

Figure 2.35: How to obtain the fractal dimension of the size distribution of companies.

the following relation between $k(n)$ and $n$ :

$$
k(n) \propto n^{D} .
$$

This exponent $D$ is the fractal dimension. If $D$ is between zero and one, that set is fractal. If $D$ is close to one, it is a line-like object, while if $D$ is close to zero it resembles sparsely distributed points. ${ }^{15}$

Figure 2.36 charts the results of the Monte Carlo calculation of the fractal dimension $D$ for various values of the Pareto index $\mu$. Since the fractal dimension is obtained more accurately for large numbers, we used data for some $10^{8}$ companies. One possible conclusion from this result is that the accurate value depends on $\mu$ as the broken line denoted by " $\infty$ ?". This line is divided by $\mu=1$ : For $\mu>1$, we find that $D=1$, and that the distribution is dense, while for $\mu<1$ we find that $D$ is smaller than 1 , and that fractality manifests itself in a Devil's Staircase.

\section{Golden ratio, Silver ratio, and self-similarity}

Both the Golden and Silver ratios have long been associated with powerful aesthetic effects.

The Golden ratio, sometimes called the golden mean, has been observed in numerous places: for example structures such as the Pantheon, and in paintings such as the Mona Lisa. The Silver ratio is said to be hidden in the pagoda of the famous Horyuji-temple in Nara, Japan, and in images of the

\footnotetext{
${ }^{15}$ We note that there are a variety of "fractal dimensions". What we have explained here is the "box dimension", sometimes called the "entropy dimension" or "capacity dimension". We have used this here because it is easy to measure (Alligood et al., 1997; Falconer, 2003).
} 


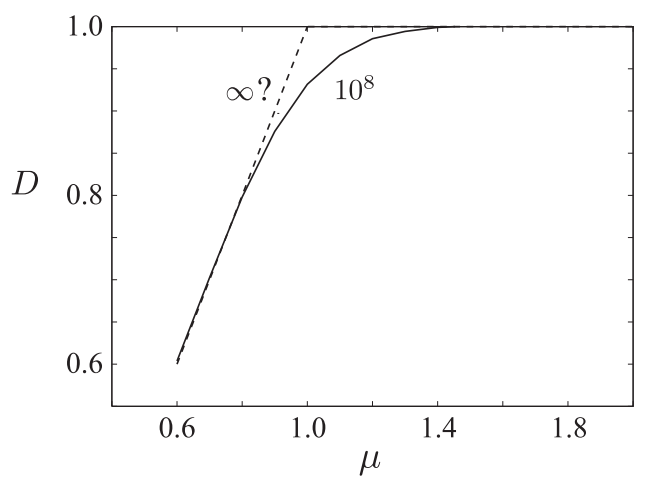

Figure 2.36: Fractal dimension of the Devil's Staircase.

faces of Buddha.

The actual value of the golden ratio is $1: \frac{1+\sqrt{5}}{2} \cong 1: 1.168$, and the silver ratio $1: \sqrt{2} \cong 1: 1.414$. Both of these have a close connection with the self-similarity which is essential to the fractality.

We show below various rectangles whose sides have these ratios. The slim rectangle on the left has a ratio equal to the golden ratio, while the larger one the right is that of the silver ratio.

If we delete the leftmost square from the left rectangle, as shown in the illustration, the sides of the remaining rectangle still have the same ratio, the golden ratio. A similar operation on this smaller rectangle yields another rectangle with the golden ratio. This goes on and on infinitely, always resulting in a rectangle with the golden ratio, a variety of self-similarity. Inversely, if we impose this kind of self-similarity on a rectangle, it automatically has the golden ratio.

On the other hand, if we fold a rectangle of the silver ratio along the middle we again obtain a rectangle of silver ratio. Folding in half again, we obtain a silver ratio again and this goes on infinitely, yet another variety of self-similarity. Incidentally, this property is used for the international standard (ISO 216) for paper size: A0 paper is a rectangle of area equal to $1 \mathrm{~m}^{2}$ whose sides have the silver ratio. Having it we obtain A1, halving A1 is $\mathrm{A} 2$, and so on. 

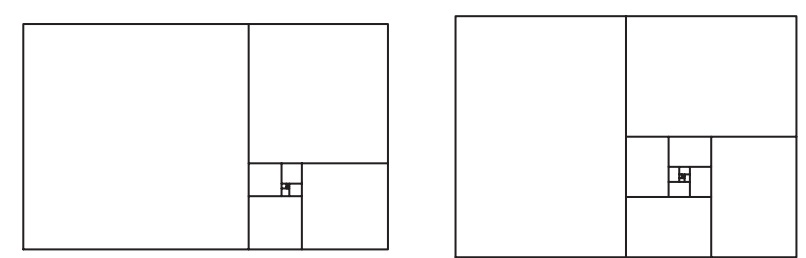

Is the infinite self-similar layer the source of beauty? Many artists and philosophers have supposed it must be, and there is a large literature on the subject. We don't know the answer, but we are very wary of explanations that try to unite very diverse phenomena on slender grounds. Is there really any reason for thinking that there will be one fundamental causal factor behind aesthetic phenomena as apparently diverse as colour combinations, facial characteristics, landscape, sculpture, music, and literature? Perhaps not. However, it may well be that self similarity is peculiarly attractive and rewarding for the human mind, and accounts for some part of some of those experiences which we categorise together, in spite of their diversity, as aesthetic.

(H.I. \& J.C.)

\subsection{Side story: "long-tail phenomena"}

Chris Anderson, the editor-in-chief of Wired magazine, ${ }^{16}$ learned of an empirical law of sales by using statistics from a digital jukebox company. This empirical law is quite different from that familiar in the relation to mega-hits (Anderson, 2006). A CEO of the company asked "What percentage of the top 10,000 titles in any on-line media store (Netflix, iTunes, Amazon, or any other) will rent or sell at least once a month?" Knowing Pareto's 80-20 principle, described in Section 2.4.5, Anderson estimated that $80 \%$ of the sales comes from $20 \%$ of the products, and so guessed at about $50 \%$. His mental image was ten thousand books at a large bookstore and ten thousand CD albums at Wal-Mart, half of which are left unsold. However, the answer from this CEO was that " $99 \%$ " of those top titles sold at least once a month!

In a world where the cost including packaging and storage is negligible and instant search and download is possible, consumers behave in a certain pattern: demand is unlimited in variety. Consequently, Anderson became interested in what endless demand and unlimited supply could bring to business and found similar phenomena in iTunes music and Amazon books.

In particular, he made a rank-size plot of one month's downloads of about one million titles from an on-line music company and found a long tail of albums that did not sell much, but whose aggregate contribution to sales is comparable to mega-hits. He described his finding in his article entitled "The

\footnotetext{
${ }^{16}$ It is interesting to the present authors that he too comes from a background in physics, and has a degree from George Washington University, and did research at Los Alamos Laboratory, before starting his career in publishing.
} 
Long Tail", which made a great impact on net-business.

There is a difference in perception here. The long tail we have so far discussed was concerned with a few giants, mega-hits, while Anderson's "longtail" is about dwarves and their sum total.

An average American movie theater needs to have at least 1,500 customers during a two-week period of play in order to stay in business, but on-line distributors, which do not need a millimeter of shelf-space, are apparently quite different. ${ }^{17}$

This "long-tail" phenomena can be understood by using the CDF, with its variable $x$ being the sales of goods or downloads as the property not of the tail that extends to the right of the plot,

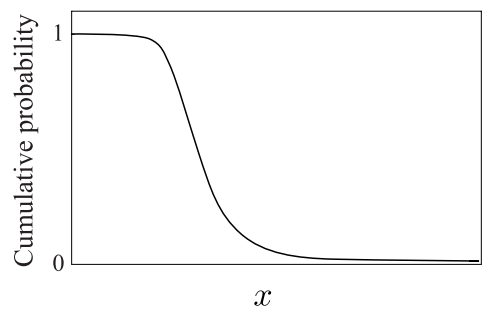

but of the region of small values of $x$ to the extreme left. The behaviour is revealed in the following plot:

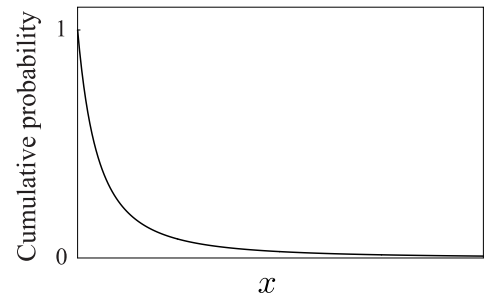

It is interesting to ask whether the power law is obeyed in the region of small values of $x$.

This sort of new business model is now attracting considerable attention, and is crossing over from the academy into the world of business. For instance the well-known microeconomist Hal Varian of the University of California Berkeley (Varian, 1992) is also a chief economist at Google. We think it's important that a wider range of companies becomes interested in new ways to examine various economic data. The mere plotting of distributions is just a start. New insights into the long tails caused by elementary processes, such as the sales of individual items, whose distributions and fluctuations are not modeled yet, may result in still more new routes leading to fresh business

\footnotetext{
${ }^{17} \mathrm{It}$ is worth noting that well before the age of the internet, some retailers like Sears were
} launching a business model with an unlimited supply. 
models. For instance, with improved knowledge of the distribution of growthrates we may be able to achieve scientific risk management of the sales of goods. ${ }^{18}$

Neither Pareto nor Gibrat could have anticipated modern internet business with all its new distributions, but their work is intensely relevant, and has prepared the way for a new world of research, bringing academics into partnership with businesses producing very large and valuable data-sets. Certainly, the present authors would welcome contacts from any enterprise interested in this kind of collaborative work.

\section{6 $\mu=1$ and Phase Transition}

As we have seen in this chapter, the Pareto index, $\mu=1$, is a very special value. Let us now look back at what we have found and think about its meaning.

We have seen that in various countries, such as France, the United Kingdom, Italy, Spain, and Japan, several quantities used to measure company size, such as total capital, sales, and the number of employees, exhibited a Pareto index very close to 1 , with only a small fluctuation around this value from year to year. This is radically different from personal income, the Pareto index of which fluctuated between $\mu=1.3$ and 2.6 (Figure 2.6). This may seem unsurprising when we recall that people tend to be more equal than companies. In mature capitalism, companies are engaged in an unremitting struggle for survival. If defeated, a company may become bankrupt or be subject to merger or acquisition. By contrast, while individuals try to achieve more prosperity they rarely try to eliminate other people financially. Therefore, it is natural to expect that people are more equal than companies, resulting in a larger Pareto index. ${ }^{19}$ If this is case, why does the Pareto index of companies stay close to 1, rather than falling below this value?

We have examined the property of Pareto distributions in Section.2.4 using the staircase plot as a guide to and a visualization of inequality. We have seen that for $\mu<1$ the staircase became quite ragged, indeed devilish, making the inequality of the society manifest even to a casual observer. On the other hand, for $\mu>1$, the staircase is disguised by the slope and the inequality is hidden behind its smoothness.

This has been quantified by the calculation of the relative shares. For values of $\mu$ smaller than 1 , even if there are infinite number of companies, the finite number of top companies have a finite share. This is the emergence of oligopoly, which wouldn't occur under a normal distribution. On the other hand, nothing like that happened for $\mu>1$. At $\mu=1$, a somewhat intermediate situation is realised. In fact, the average top share was almost inversely

\footnotetext{
${ }^{18}$ We are a bit ahead of our story here: we will elaborate on growth rate in detail in the next chapter, where Gibrat's Law will play an essential role.

${ }^{19}$ However, it should be noted that several studies of business sectors where governmental control is tight, such as the electric power industry, the distribution is known to be far from a Pareto distribution, namely $\mu=1$.
} 
proportional to logarithm of the number of companies, $N$, and very slowly goes to zero as $N$ diverges. Further, in this case the shape of the Devil's Staircase was sometimes smooth and sometimes rough, varying a lot from dataset to dataset.

We also saw that both Gini's coefficient and the Robin Hood index showed that oligopoly does occur for $\mu<1$, and that a self-similar fractal appears for $\mu<1$.

All these show that the Pareto index $\mu=1$ is the very boundary between the oligopolistic state and other more equal states, which we might call pseudo-equal states.

Borrowing terms from Modern Statistical Physics, this kind of a point may be called a transition point, or a critical point.

The majority of economists and business people know little of contemporary physics and its analytical tools and modeling methods. We think that this is a pity, not least because, as we discussed earlier history tells us that the disciplines of physics and economics have long been connected. But the real value is that even a modest grasp of physics may yield substantial improvements in understanding of the character of economic and social states.

Let us consider heating water at a location close to sea level. If we fill a kettle with water and start to heat it, the temperature rises. As it approaches 100 degrees Celsius, the water will start to boil. Further heating at this stage does not raise the temperature, but only results in more vapour. The water molecules in the vapour obtain their kinetic energy from the heat and are able to exit the liquid state. So any additional heat is used to make vapour, and is carried away by those molecules. However much more energy you put into the water the temperature of the water does not rise further.

In cases such as this the water molecules may exist in a liquid state, water, or can be in a gaseous state, vapour (or a solid state, ice). These states are called a phase. The liquid state is the "liquid phase" and the gaseous state the "gaseous phase". A change of water to vapor, or vice versa, is called a phase transition.

The point $\mu=1$ for Pareto distributions is the critical point between the oligopolistic phase and the pseudo-equal phase. The economic society of companies is, so to speak, boiling at this critical point.

There are two forces that drive the economic society of companies towards this critical point $\mu=1$. Companies, especially large companies, try to grow and to obtain large profits at the expense of their competitors. This drive towards yet larger companies forces the value of $\mu$ to decline. On the other hand, the oligopolistic phase is in some senses undesirable, as it obstructs free competition and may distort the market. Antitrust laws exist in many countries to suppress this kind of economic state, and at the worst case, large companies are split into smaller companies. There are a number of other governmental measures to suppress oligopoly and monopoly. All these work to push up the value of $\mu$, but only up to 1 , as above $\mu=1$ oligopoly essentially vanishes. Therefore these two competing pressures enforce $\mu=1$, as illustrated in Figure 2.37. This is why the actual Pareto index in mature 


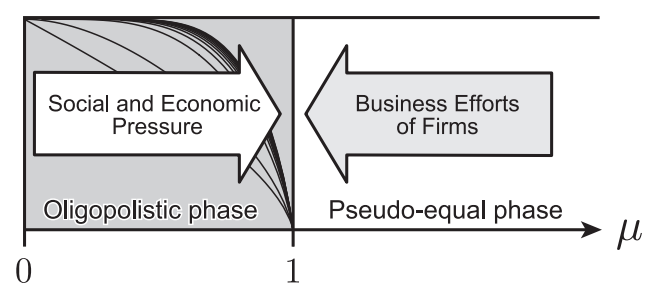

Figure 2.37: Two forces that besiege $\mu=1$.

capitalistic countries hovers around $\mu=1$.

When heating water, added thermal energy is transferred to kinetic energy of water molecules. Those molecules collide with each other, which results in the exchange of a part of their kinetic energy. As a result, the kinetic energy of a water molecule obeys an exponential distribution called a Boltzmann distribution. Since molecules keep colliding with each other, the kinetic energy of each molecule fluctuates. But as the number of molecules is very large, the group of molecules as a whole obeys the Boltzmann distribution and other elegant statistical laws. ${ }^{20}$

We think that many readers will be thinking of the connections by now: "molecules" ... "companies"? "collisions" ... "business transactions"? That, in a nutshell, is the analogy between boiling water and companies. Since all the agents that make the society, individuals, companies, banks, markets, and government agencies, are diverse and differ considerably in their functions, one may think it is impossible to find any law to describe them all. However, there are so many of them, each influencing the others through trade, stock exchange and other financial transactions, the exchange and sharing of personnel, and so forth, that a single economic society is the result, just as the myriads of interactions between water molecules produces a phenomenon susceptible of scientific explanation. We suggest that, in fact, some statistical laws are indeed valid for the whole, and in this chapter, we have tried to show that there are some statistical laws governing the group of all companies.

Water molecules interact with each other in three-dimensional continuous space. On the contrary, heterogeneous economic agents interact with each other on a network, such as a transaction network, a stock-holding network, or a personnel-sharing network. Economic society is made of layers of these various networks overlapping with each other. Our next task, then, is to look at these network structures and the interaction between companies, and so to arrive at a scientific vision of modern-day economic society.

\footnotetext{
${ }^{20}$ We note that Aoki and Yoshikawa (2007) contains a theory that predicts the productivity distribution to be a Boltzmann distribution, which was further developed in the framework of superstatistics by Aoyama et al. (2008).
} 


\section{Chapter 3}

\section{Fluctuations - Company Growth}

In the preceding chapter we examined the statistical laws and patterns observed in distributions of company-sizes, and it was noted that the tails of the distributions of flow and stock variables quantifying company-size both obey Pareto's law.

Indeed, as was mentioned in the Section 2.1.2, Pareto's power-law is commonly found in many phenomena observed within natural science, as well as the social and economic sciences. Most researchers in these fields would accept that there is no single mechanism giving a universal explanation for all these phenomena, but rather that different mechanisms would be found for each of a variety of classes of phenomena. ${ }^{1}$

Company-size obviously changes over time: last year's sales are not equal to this year's; the previous quarter's total assets is different from that in this quarter; and debt increases or decreases according to levels of borrowing from financial institutions or from investors. Thus we can see that observations on the distribution of company size are an instantaneous snapshot of the state of a collection of companies, each of which is individually subject to fluctuations.

Our analysis is now being led towards the examination of temporal change of company-size in terms of flow and stock variables, with a view to providing an account of the appearance of size distributions seen as a consequence of fluctuations. Therefore, our next step is to understand fluctuations in company-size, in other words the growth or contraction of companies. ${ }^{2}$

The following chapter's focus on temporal change of flow and stock variables relating to company-size is structured as follows.

\footnotetext{
${ }^{1}$ Mechanisms explaining the origins of power-laws in natural and social sciences have been uncovered for more than a dozen classes of phenomena. See Sornette (2004) and references therein.

${ }^{2}$ The importance of understanding fluctuations in terms of heterogeneous interacting agents is well covered in recent studies (Aoki, 2002; Aoki and Yoshikawa, 2007; Delli Gatti et al., 2008)
} 
First, we show that another factor called Gibrat's law is phenomenologically valid by examining it in large datasets for European and Japanese companies. In the course of this work we reveal a remarkable fact, an effect which we will call detailed balance, which represents a sort of stability in the growth of companies, and is key to relating Gibrat's law (dynamics) to Pareto's law (statistics).

In addition, we discuss an important statistical method, namely the copula method, which can be employed as an alternative to growth rate when measuring the change between two variables.

Secondly, we will make a detour through the subject of personal-income distribution, and reconsider Pareto's original finding, his famous Law, in conjunction with Gibrat's law, and both in the light of "detailed balance". Interestingly, we will find the same dynamical structure that is apparent in company-growth, but more importantly, it will be shown that those laws can break down under abnormal situations in a nation's economy, such as the collapse of the Japanese "bubble economy" in 1990.

So far our story has been concerned with and applies to large companies, which are dominant in the aggregated sum of sizes and fluctuations. On the other hand, small and medium-sized companies prevail in overall number. Fortunately, the financial institutions responsible for credit risk management in Japan have recently accumulated and published very large datasets concerning small and medium-sized companies, enabling us to study distributions and fluctuations in this area and reveal phenomena not covered by Pareto's laws. Indeed, we shall find a distinctive boundary between large and small companies based not on the legal regulations or arbitrary definitions of what are large and small companies, but instead on quantitative differences in the statistics and dynamics. This observation is our third major point.

Fourthly, in contrast to "growth", it is possible for a company to pass into a state of "death". Recall that a company's activity is aimed at the profit anticipated from sales over and above expenses such as labour and financing, and thus that the profit will be determined a posteriori by several factors such as sales fluctuations, costs, the price of intermediate goods, interest rates, and risks due to exchange-rates. If the company becomes short of retained earnings because of those factors, it goes into bankruptcy, or experiences corporate death.

Finally, it would be of crucial importance in such activity for a company to determine the amount of output by deciding the input of capital (nonhuman resources) and labour (human resources). How can we understand the collection of those activities in terms of microscopic behaviour, in which each company attempts under various constraints to maximise profit? We discuss this point briefly under the terms production function and what we call the ridge theory of company growth. 


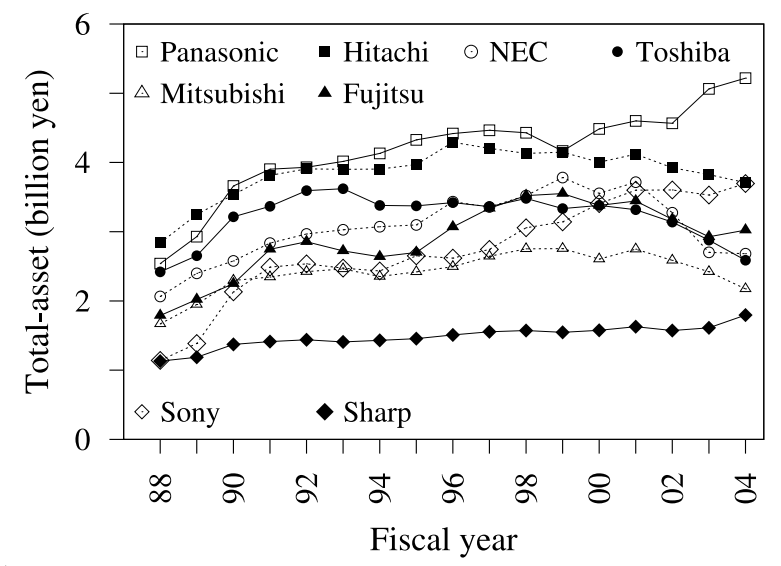

Figure 3.1: Time-series of annual company-sizes for the eight largest electronics and electric-machinery companies (1988 to 2004).

\subsection{Gibrat's Law and Detailed Balance}

Let us begin and take a look at real data so as to understand important phenomenological facts concerning the fluctuations of company growth. ${ }^{3}$

\subsubsection{Growth Rate and Gibrat's Law}

The growth of company-size for flow or stock variables can be easily quantified by measuring the quantities at different points in time. To illustrate this, in Figure 3.1, we depict the time-series of annual company-sizes (defined by the summation of assets and debt) for the eight largest electronics and electricmachinery companies (Fujitsu, Hitachi, Mitsubishi, NEC, Panasonic, Sony, Sharp, Toshiba) in Japan, from the fiscal year 1988 to $2004 .^{4}$

The time-scale here is on an annual basis for illustrative purposes; in fact, a quarterly or monthly basis would be adequate. The point is that the dynamics of company growth can only be understood with a time-scale that is longer than that of the activity of the company, typically days and weeks.

In addition, the time-scale should be shorter than that over which macroeconomic conditions surrounding the companies are subject to change, say,

\footnotetext{
${ }^{3}$ There exists a huge literature on company, industry and country growth in economics. We refer to Amaral et al. (1997, 1998); Bottazzi and Secchi (2003); Fu et al. (2005); Gabaix (2008); Ijiri and Simon (1977); Okuyama et al. (1999); Stanley et al. (1996); Steindl (1965); Sutton (1997); Takayasu et al. (1997) and references therein for other aspects and introduction to previous studies.

${ }^{4}$ The data is on an unconsolidated basis. Companies have slightly different policies in accounting for consolidated and unconsolidated data, so a simple comparison of values for company-sizes might be inappropriate here.
} 


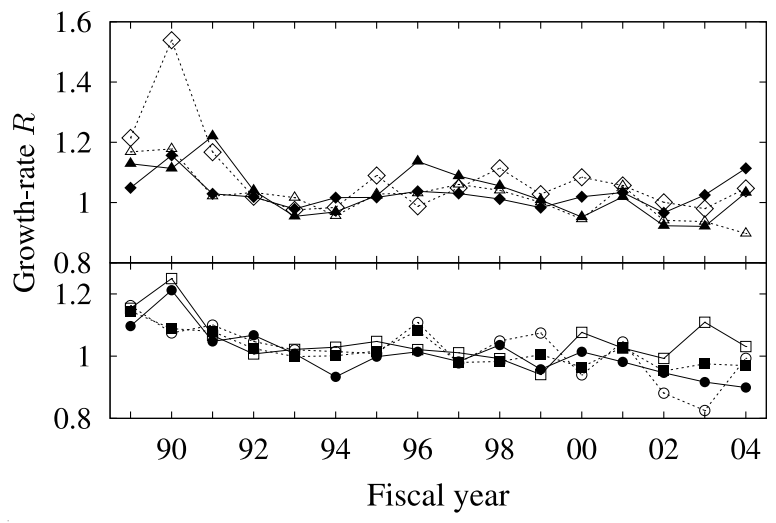

Figure 3.2: Time-series of growth-rates for the eight major electronics companies (corresponding to Figure 3.1; 1989 to 2004).

years and decades. Otherwise, we would not be able to separate the "fast" variables of company-level dynamics from the "slow" variables of nation-level macroscopic dynamics. ${ }^{5}$

Let us denote by $x_{1}$ and $x_{2}$ a company's sizes at time $t_{1}$ and a succeeding time $t_{2}$ respectively. We can further define growth-rate by

$$
R=\frac{x_{2}}{x_{1}}
$$

The variable $R$ represents the ratio at which a company increases or decreases its size, such as sales and total-assets, in the time interval. In addition, we will define the logarithmic growth-rate as

$$
r=\log _{10} R=\log _{10} x_{2}-\log _{10} x_{1},
$$

where $\log _{10}$ is a logarithm with base $10{ }^{6}$ If the size does not change, the growth-rate is $R=1$ and the logarithmic growth-rate is $r=0$; if the size increases, $R>1, r>0$ and if it decreases, $R<1, r<0$.

Figure 3.2 shows the annual growth-rates calculated from Figure 3.1. (The upper and lower panels divide the eight companies into two groups of four simply for visual ease.) It can be seen that the growth-rates are diverse across the companies and over time vary around the value 1 . If we think of individual

\footnotetext{
${ }^{5}$ It is not trivial, a priori, that such a separation of time-scales is possible. Some abnormal situations, caused by a global shock, for example, may not allow one to distinguish between time-scales in this way. It would, nevertheless, be reasonable to assume that it is possible in ordinary instances, and thus to observe consequences which can be tested against empirical facts a posteriori

${ }^{6}$ For non-math readers: when $y=10^{x}$, one writes $x=\log _{10} y$ by definition. E.g., $\log _{10} 1=0, \log _{10} 10=1, \log _{10} 100=2, \ldots$
} 


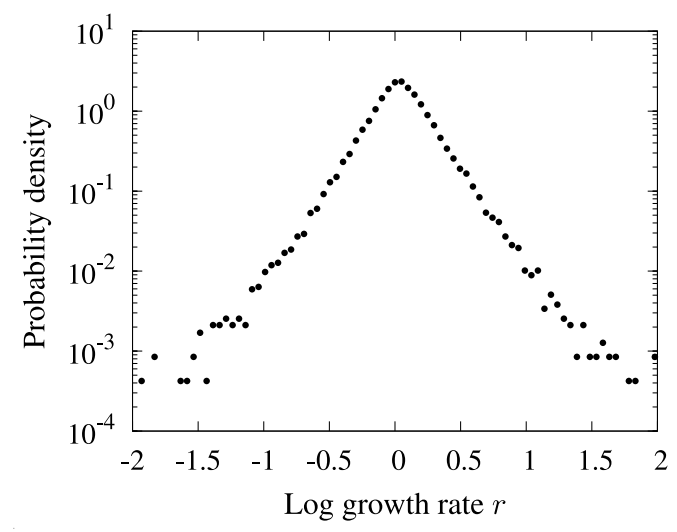

Figure 3.3: Probability distribution for logarithmic growth-rates of company-income (2001 to 2002; roughly 57,000 companies).

companies at the same instant of time as spatially related, we can say that growth-rates fluctuate both in space and time.

Since this spatial fluctuation is related to company-size distribution, we can now proceed to investigate the spatial distributions of growth-rates.

We can begin by using the flow variables of companies' declared incomes in the Japanese dataset, the distribution of which was shown in Figure 2.4. This is a suitable set since it is an exhaustive list of incomes for about 57,000 companies covering the region where the power-law holds. We can calculate the growth-rate for each company for two successive incomes from the year 2001 to 2002. Figure 3.3 is the PDF as a histogram for the logarithmic growth-rates $r$. The magnitudes $r=0.5$ and 1 correspond to the increase by $3.2\left(\approx 10^{0.5}\right), 10$ times, while $r=-0.5$ and -1 represent the decrease by $0.32\left(\approx 10^{-0.5}\right)$, and $1 / 10$ times, respectively, in terms of $R$.

Note that the ordinate is a logarithmic scale. The growth-rate distribution has a strong peak at $r=0$, and declines as an exponentially decreasing function on each side of positive and negative values of $r$. The number of companies increasing or decreasing in size by a multiple of three $(R=3$ or 0.3 ) can be roughly estimated to be $10 \%$ of the number of companies with no change $(R=1)$; also the number for increase or decrease by a multiple of 10 is roughly $1 \%$ of the number for unchanged companies. It is intuitively remarkable that a company as large as $R=2$ or $R=0.5$ could double or halve in a year, but in fact the data shows that this is a quite dramatic observable change.

It is evidently reasonable to ask whether growth rate is dependent on company size, for example we might ask whether the growth-rate of a company of size 1 in an arbitrary scale is large when compared with that of a com- 


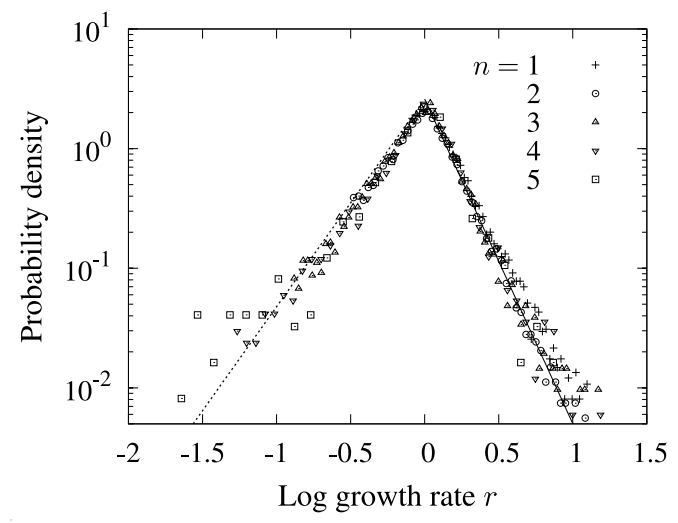

Figure 3.4: Probability distribution for logarithmic growth-rates conditioned by company income size (corresponding to Figure 3.3).

pany of size 2? To answer this question we can examine the distribution of sizes in Figure 2.4 on page 21 again. Let us segregate those companies into categories (or bins) by dividing the size range into five logarithmically equal bins, covering incomes of 50 million up to 5 billion yen. In other words, we will divide the interval between two orders of magnitude (from 50 million to 5 billion) into bins by multiples of $10^{2 / 5}=10^{0.4} \approx 2.5$; the first bin ranges from 50 million to $10^{0.4} \times 50$ million, roughly 125 million, and similarly for the others. ${ }^{7}$

The resulting bins are then

$$
\left[5 \times 10^{10+0.4(n-1)}, 5 \times 10^{10+0.4 n}\right] \quad(n=1, \ldots, 5)
$$

We can examine the PDF for the logarithmic growth-rate $r$ by choosing those companies whose sizes fall into each bin. This is called a conditional PDF. Figure 3.4 depicts in a single plot the conditional PDFs thus obtained.

What can we conclude from this plot? Obviously the calculated PDFs overlap and form a single curve as shown in Figure 3.4 (or equivalently as in Figure 3.3). The growth-rate does not depend on the selected bin, i.e. it is independent of the company size at the outset, as the distribution in the figure shows.

In other words, for companies in the regime of Pareto's law, the fluctuation in their rise and fall, as quantified by growth-rate, is statistically independent of the size.

This fact looks quite strange at first sight, for one might expect a quantitative difference in the fluctuations for group A and B of companies with differ-

\footnotetext{
${ }^{7}$ One could simply split the range into linearly equal bins, but since we know that the tail of the distribution exhibits a power law, this would make an inefficient use of the data.
} 
(a) Growth of sales (yr2002/2001)

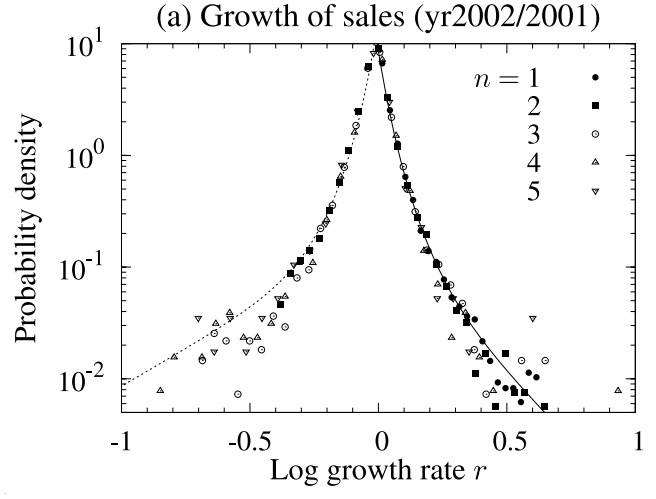

(b) Growth of profit (yr2002/2001)

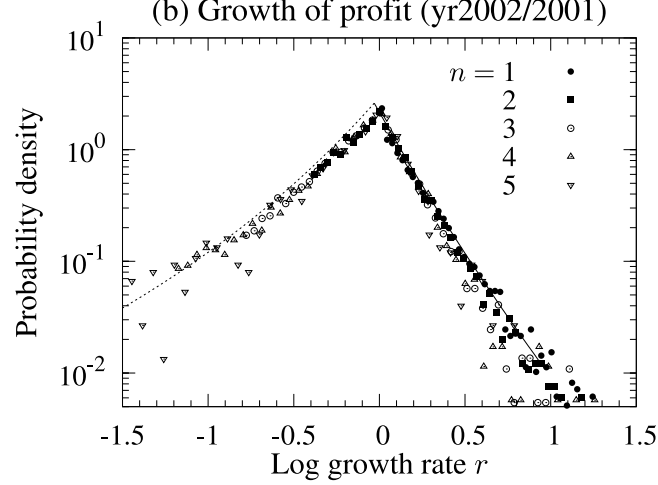

Figure 3.5: Probability distribution for growth-rates ((a) sales and (b) profits for the years 2002/2001).

ent sizes, a difference that would be responsible for the diversity of companiessizes between $\mathrm{A}$ and $\mathrm{B}$.

However, our finding is different, and we shall refer to this non-trivial fact as Gibrat's law.

\subsubsection{Data for Japanese Companies}

So far, we have examined an exhaustive list of companies from a Japanese company-income dataset, and found Gibrat's law. It is interesting to ask whether this law also holds for the other quantities that can characterise company activity.

For example, we could take the dataset used for our study of company-size distribution, which covers hundreds of thousands of companies, and examine 
the sales and profits (positive profits, not deficits, are considered here).

Figs. 2.9 (a) and (b) show the annual growth-rate for the years 2001 and 2002 for sales (0.34 million companies) and for profits ( 0.27 million), respectively. The PDFs are conditional, just as they were in Figure 3.4, though the condition is imposed on the magnitude of sales or profits.

The indices $n=1, \ldots, 5$ correspond to five logarithmically equal bins, ranging from 2 billion to 2 hundred billion yen for sales, and from 20 million to 2 billion yen for profits.

We observe that the distribution of growth-rate differs from one variable to the other. The growth-rate for profit has a larger width in its distribution than that for sales, and a narrower peak around $r=0$. We can see, nevertheless, that the PDFs overlap in a single curve irrespective of the chosen bin $n$. Thus, Gibrat's law also holds for these variables.

\subsubsection{Data for European Companies}

Sales and profits are flow variables, as opposed to stock variables such as totalassets, the number of employees, and others, so we might further ask whether Gibrat's law applies to this latter class of characteristics of company-size, and whether it applies in countries other than Japan.

To shed light on these questions we will now turn to several results from datasets for European companies used in Section 2.3.2. The criterion used for selecting companies for inclusion in the datasets are as follows: profits are larger than 15 million euros, or total-assets exceed 30 million euros, or the company has more than 150 employees. See Fujiwara et al. (2004) for details.

In Figure 3.6 (a) (b) and (c) are the distributions for annual growth-rates of total-assets and sales in France, and of the number of employees per company in UK, using the same method of conditional PDFs as employed in Figure 3.4.

The bins employed, $n=1, \ldots, 5$, are 30 million to 3 billion euros for totalassets, 15 million to 1.5 billion euros for sales, and 150 persons to 15 thousand for the number of employees, in logarithmically equal intervals.

It can be seen that the distributions for different variables have different shapes, and that the width of fluctuation in the number of employees is relatively small compared with that in total-assets. The time-scales involved in the fluctuation and dynamics of those variables are different, but we can see, simultaneously, that the PDFs give a single overlapping curve whichever bins, $n$, are observed. Thus we have established that Gibrat's law holds quite universally, irrespective of variables and countries. 
(a) France total-assets (yr2001/2000)
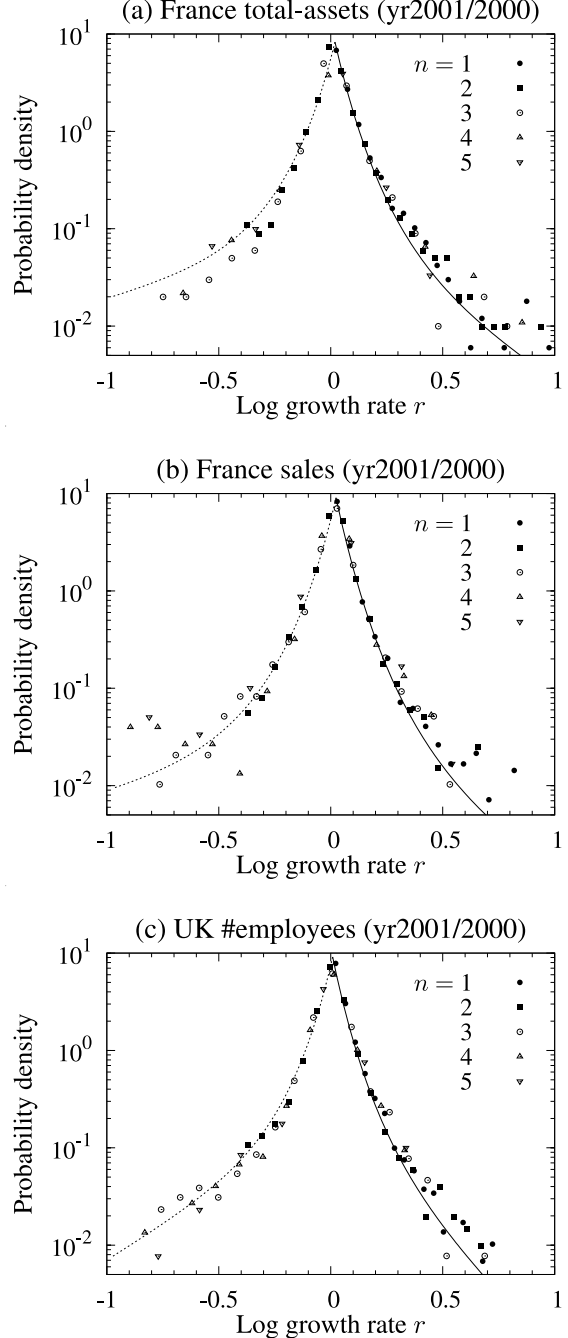

Figure 3.6: Probability distribution for growth-rates ((a) totalassets (companies in France), (b) sales (France), and (c) number of employees per company (UK) for the years 2001/2000.) 


\section{$\alpha_{\beta} \gamma$ Mathematical expression of Gibrat's law \\ If the conditional PDF for the growth-rate $R$ of the company-size $x$ is denoted by $q(R \mid x)$, Gibrat's law can be expressed as

$$
q(R \mid x)=q(R),
$$ \\ that is, $R$ 's independence of $x$ for a certain range of $x>x_{0}$. \\ The PDFs shown in Figure 3.4, Figure 3.6 and elsewhere depict the probability distribution function for a logarithmic growth-rate $r=\log _{10} R$, which is related to that for $R$ by the equality of probability, $q(r) d r=q(R) d R$.}

\subsubsection{Gibrat Revisited}

Readers in a tearing hurry may want to skip this section, where we pause to consider Robert Gibrat (1904 - 1980) a French researcher in economics and the origin of the law.

In his seminal treatise, "Les Inégalités Économiques" (Gibrat, 1931), Gibrat proposed a stochastic process which offers the potential of explaining the dynamics of company-size. At the time of writing, researchers had found distributions with heavy-tails in various disciplines of natural science as well as social and economic sciences, amongst them Jacobus Kapteyn, an astronomer who had discovered the rotation of the Galaxy. Kapteyn had suggested a simple process as one of the possible mechanisms that might bring about a highly skewed distribution, and as a result of correspondence with Kapteyn, Gibrat turned his mind to the origin of economic and social inequalities (see Sutton (1997)).

The stochastic process is quite simple. Consider a dynamical variable $x_{t}$ at time $t$ and its growth $R_{t}$ which is independent of $x_{t}$ and temporal statisticalindependence, i.e., at each time the growth simply takes a value drawn from a distribution. He called the basic idea in this stochastic process a "law of proportionate effect", and actually placed this expression in the subtitle of the treatise. "Proportionate" refers to the multiplicative nature of the process, that is, the size at the next time-step is determined by a multiplication of the previous size and an independent multiplicative factor (see Redner (1990) for instance). We shall call this Gibrat's process.

We will later see that if we run a simulation for this stochastic process, the result is a so-called "log-normal" distribution, one of the classes of skewed distributions well-known in physics and the social sciences (see Aitchison and Brown (1957)). One can prove, in addition, that the average and variance of the distribution grow in time. Now, Gibrat employed data of personal income and company-size (measured by number of employees, factories, etc.)

\footnotetext{
${ }^{8}$ The subtitle is long; applications: aux inégalités des richesses, à la concentration des entreprises, aux populations des villes, aux statistiques des familles, etc., d'une loi nouvelle, la loi de l'effect proportionnel. The phrase "the new law" may be read as a strong claim with regard to his endeavor to explain many social phenomena such as personal income, companies, cities, etc.
} 
available at the time of writing, and actually fitted their distributions by lognormal distributions claiming the stochastic process as a new finding, a new principle or a law.

Revisiting Gibrat's study with today's high-precision and large-scale data, we immediately notice the difficulties:

- The log-normal distributions do not fit well for the region of major companies of large size which have studied in his work, putting aside the region of small and middle-sized companies.

- The average and variance do not grow over time.

The data employed in Gibrat's study were not only limited in extent, but were presumably to some degree biased, with the result that the apparent fit to the log-normal distribution and its mathematically well-studied model, led Gibrat into concluding that this was what should be considered first. In regard to the second point, Gibrat himself recognised the difficulty and tried, without success, to provide possible solutions (see Sutton (1997)).

Let us recall that we denominated as Gibrat's law the following property: let the growth-rate of a company $i$ at a point in time be denoted by $R_{i}$; if the probability distribution for the collection of growth-rates $\left\{R_{i}\right\}$ is statistically independent of the company-size $x$, then the law holds.

Gibrat's law itself does not lead to the Gibrat's process. It will be instructive to exemplify and emphasise this point.

Consider an example of Gibrat's process, that is, a multiplicative process as described above (see page 169). Suppose a company follows this process, then it can continue its activity of business however small it may be, because the succeeding multiplicative factors may happen by chance to be small. To avoid such an unrealistic situation, let us assume that there is a threshold for the size of company, which is interpretable as the minimum size of assets, facilities, and number of employees necessary for survival. Also suppose that a company exits the field of business, and is replaced by a newcomer. The new company could start with a minimum size as we have already assumed. This can be conceived of as a "reflecting barrier", positioned at a small value for company-size; the barrier repels companies shrinking down to the threshold and propels them in the direction of growth in size.

This modifies Gibrat's process slightly, but obviously satisfies the property of Gibrat's law as defined above. Moreover, if we were to run a computer simulation for a collection of companies, each of which follows the modified process, we would find that the distribution for company-size follows a Pareto law in the tail. Mathematically, it can be proved that a Gibrat process with a reflecting barrier boundary condition obeys Pareto's law (see Sornette and Cont (1997)).

However, we do not claim here that this is a plausible model for explaining the dynamics of companies, at least with respect to the Pareto's and Gibrat's laws. Nevertheless, we can now see that Gibrat's law itself does not lead to 
the Gibrat process, which is just one of the many possible models that satisfy the property of Gibrat's law.

Even so, this observation does not contradict the law of proportionate effect which we refer to here as Gibrat's law. On the contrary, Gibrat's insight was to focus on the statistical independence of growth-rate, and this turns out to be correct. ${ }^{9}$

The point is that Gibrat's process was simply not an appropriate model to describe real dynamics, as researchers have found with the help of abundant empirical data. We need to find another key to understanding the kinematics of the rise and fall of companies, and this will be attempted in the following section, where we will introduce the concept of "detailed balance", a concept drawn from statistical physics. Together, these two key concepts - Gibrat's law and detailed balance - solve the long-standing problem of the origin of Pareto's law, eighty years after Gibrat's finding.

\subsubsection{Detailed Balance}

So far we have been using the variables, $R$ and $x_{1}$ for the description of growth-rate, but we could also use another equally valid pair, for example, $x_{1}$ and $x_{2}$. A brief glance at the pattern of fluctuation in terms of this pair of variables will illustrate this point.

It will suffice to examine how companies with size $x_{1}$ at time $t_{1}$ make a transition to a region of $x_{2}$ at time $t_{2}$. We will take all the companies in the Pareto region, and first put points for the pair $\left(x_{1}, x_{2}\right)$, resulting in a socalled scatter-plot. Figure 3.7 (a) and (b) are scatter-plots which correspond to Figure 3.5 (a) and (b) respectively. Similarly Figure 3.8 (a), (b) and (c) correspond with each of Figure 3.6 (a), (b), and (c).

Consider, in such a scatter-plot, a portion of a rectangle spanning $x_{1}$ to $x_{1}+d x_{1}$ and also $x_{2}$ to $x_{2}+d x_{2}$, and then calculate the number of companies which fall into the rectangle and divide this by the total number in the entire plot. The calculated quantity as a function of the point $\left(x_{1}, x_{2}\right)$ is called a joint probability denoted by $p_{12}\left(x_{1}, x_{2}\right) d x_{1} d x_{2}$. Here $d x_{1}$ or $d x_{2}$ represents a small interval in the direction of $x_{1}$ or $x_{2}$.

Even to the naked eye a significant feature is visible, namely that the distribution of points looks almost symmetrical with respect to the diagonal line (running from south-west to north-east).

If it is actually symmetrical in a statistical sense, ${ }^{10}$ we can say that the following fact holds: companies make a transition from a neighbour of $x_{1}$ in size (state of $x_{1}$ ) to a neighbour of $x_{2}$, and vice versa, from the state of $x_{2}$ to $x_{1}$. The frequency in the transition of companies from $x_{1}$ to $x_{2}$, and that for

\footnotetext{
${ }^{9}$ We will assume that the reader who has followed this discussion is now comfortable with this distinction, though even some professional researchers seem to confuse Gibrat's law with the Gibrat process associated with log-normal distribution.

${ }^{10}$ While it looks symmetrical, a careful statistical analysis is necessary to check the symmetry, since our eyes easily fail to identify the density of a cloud of points.
} 

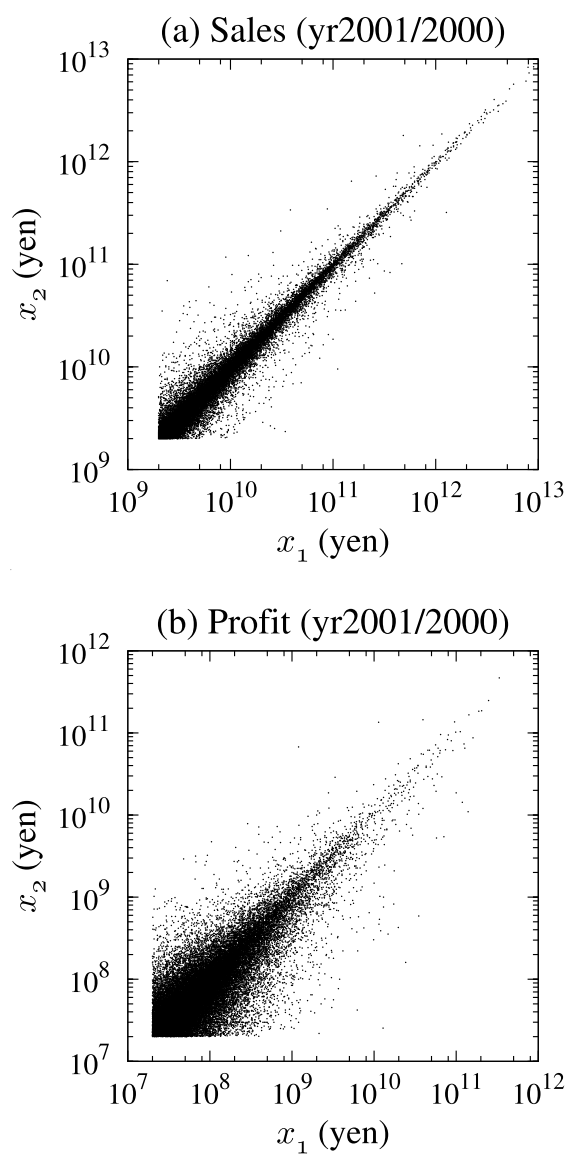

Figure 3.7: Scatter-plot for company-sizes at successive points in time ((a) sales and (b) profits for the years 2001/2000). 

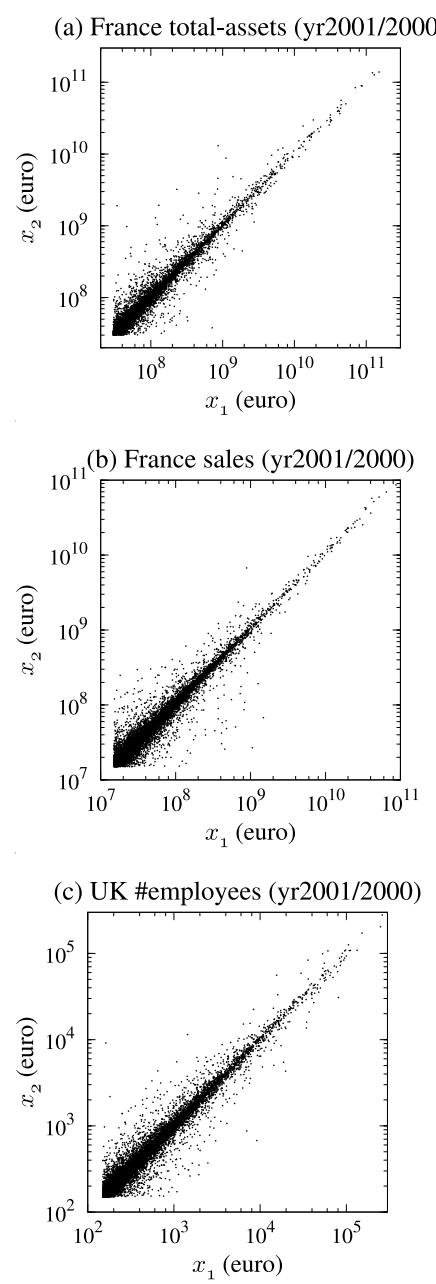

Figure 3.8: Scatter-plot for company-sizes at successive points in time ((a) total-assets (France), (b) sales (France), and (c) number of employees (UK) for the years 2001/2000.) 
the reverse transition is the same. This property is a kind of balance, which holds for any pair of states $\left(x_{1}, x_{2}\right)$ in the Pareto region.

In the study of the motion of molecules in a gas, and in other cases, statistical physics has a concept for this property, which is called detailed balance, a term which we shall consequently adopt in this book.

One way to show whether this property holds or not in actual data is to conduct a direct statistical test. Take a scatter-plot for $p_{12}\left(x_{1}, x_{2}\right)$ and flip it so that the $x_{1}$ axis is replaced with $x_{2}$, and then compare the flipped plot with the original one. The statistical test is to check if the distribution of points in these two plots is indistinguishable merely as two realisations (random samplings) of the same joint probability. A two-dimensional Kolmogorov-Smirnov test can be used for this. ${ }^{11}$ The null hypothesis, namely the symmetry in the scatter-plot, survives this test for most datasets. ${ }^{12}$

\section{$\alpha_{\beta}^{\gamma}$ Mathematical expression of detailed balance}

\section{Detailed balance can be expressed for the joint probability $P\left(x_{1}, x_{2}\right)$ as}

$$
p_{12}\left(x_{1}, x_{2}\right)=p_{12}\left(x_{2}, x_{1}\right),
$$

that is, the joint probability function is symmetric with respect to the exchange of the two arguments in the function.

The other way to check the validity of detailed balance is, as explained in the next section, to compare the data with one of the consequences of detailed balance and Gibrat's law, which we shall call a reflection law.

\footnotetext{
${ }^{11}$ To be precise, one should subtract the overall average in the growth-rate. For example, in Figure 3.2, there are trends at epochs in time, shifting the values of growth-rate to either the positive or negative side. We need to subtract such a shift of the average before conducting the statistical test, because the detailed balance we want to study here is a different property from the effect of a trend.

${ }^{12}$ The profit data in 3.7 (b) is an exception, the probable reason being a problem in the dataset itself, as discussed in Section 2.3.1.
}

\section{Two-dimensional Kolmogorov-Smirnov test}

The Kolmogorov-Smirnov test for a one-dimensional distribution is wellknown and widely used. This is a non-parametric test (no parameters are assumed in the distribution) to check if, for a one-dimensional case, the "two sets of samples are from the same distribution". In multi-dimensional cases no non-parametric test is available. Astronomy requires such a twodimensional test, because researchers want to test whether the distribution of stars differs in two different directions (samples) in a sky (of two-dimensions). Empirically, astronomers have found a statistical test which depends only on the correlation coefficient (see the column on page 121) for a two-dimensional distribution. See Press et al. (1992, Chap. 14) and references therein. 
It is a surprising fact that such a property holds for the stochastic transitions of company-size in competing companies, and we might regard it as a kind of balance or stability. In addition, we should remark that the condition of detailed balance is a law which is independent of Gibrat's law. It is easy to see that in the ecology of growing and shrinking companies, some become dominant and occupy niches vacated by falling companies. However, and this should be emphasised, detailed balance is not a mere balance for the entire population of companies, but a more stringent mathematical statement about the stochastic transitions of companies.

\subsubsection{Relation between Pareto's and Gibrat's Laws and the Reflection Law}

To sum up: we have so far shown that in the phenomenology of fluctuations, and for a wide range of company sizes, the following hold:

- Pareto's law for the distribution of the company-size

- Gibrat's law of the growth-rate of company-size

- Detailed balance in the process of growth

The question that faces us now, is how to relate these three laws to each other? A detailed argument is put in the mathematical section below, but we can anticipate the conclusion, by stating that there is actually a provable relation:

\section{Under the condition of detailed balance, Gibrat's law leads to Pareto's law as the resulting distribution of company-size}

As a bonus from the proof, it follows that what we call the reflection law also holds, which can be written as

$$
q(R)=R^{-\mu-2} q\left(R^{-1}\right) .
$$

This equation states that in the PDF for the growth-rate the functional form in the side of $R>1$ has a mathematical relation to that in the side $R<1$, and that the relation depends on the Pareto index $\mu$. Note that this reflection law alone does not determine the shape of the growth-rate's PDF. Actually we have seen that the PDFs for different variables measuring company-size take various shapes (see Figure 3.4 for company-income and Figure 3.6 for comparison).

It is implied from the reflection law that it is possible to know the distribution of growth-rate for the group of shrinking companies once one knows the distribution for the rest of the growing companies. We have actually drawn a curve in the PDFs depicted so far by first fitting a nonlinear function to the data in the positive logarithmic growth-rate $(r>0$, equivalently $R>1)$ and then by calculating the other side of negative growth $(r<0$ or $R<1)$ from 
the reflection law. Figure 3.4 and Figure 3.6 confirm that the fit is satisfactory, and the reflection law works adequately.

If you are practitioner, these laws might appear to be distracting, even boring, mathematics, and certainly far from intriguing. In fact, they have implications for the practice of business. Knowing the details in the distribution for the growth-rate of companies is of value to risk management, since one can perform a quantitative estimation of the risk associated with the growth or decline of companies. It would, of course, be necessary to take into account the consequences of Gibrat's law, that is, the independence of growth from company-size. If you make investments in a set of many enterprises, it would be prudent to consider the reflection law relating positive to negative growthrates, which in turn requires you to have the distribution for growth-rate and also to measure the Pareto index in a statistically reasonable way. That is to say, these points might prove useful in the risk management toolbox.

\section{$\alpha_{\beta} \gamma$ Proof of mathematical relation between the laws}

It is not difficult to show that Gibrat's law on page 74 and the detailed balance on page 79 lead to Pareto's law (see page 20) (Fujiwara et al., 2004).

Since the pair of variables $\left(x_{1}, x_{2}\right)$ and that of $\left(x_{1}, R\right)$ are related by the change of variable, $R=x_{2} / x_{1}$, one can easily see that the joint probability distribution $p_{1 R}\left(x_{1}, R\right)$ is related to the joint probability distribution $p_{12}\left(x_{1}, x_{2}\right)$ by

$$
p_{12}\left(x_{1}, x_{2}\right)=\frac{1}{x_{1}} p_{1 R}\left(x_{1}, R\right) .
$$

Now, the conditional PDF $q\left(R \mid x_{1}\right)$ for the growth-rate satisfies

$$
p_{1 R}\left(x_{1}, R\right)=q\left(R \mid x_{1}\right) p_{1}\left(x_{1}\right),
$$

by definition, where $p_{1}\left(x_{1}\right)$ is the PDF for the size $x_{1}$.

Assume that detailed balance holds, then the first equation yields

$$
p_{1 R}\left(x_{1}, R\right)=\frac{1}{R} p_{1 R}\left(R x_{1}, R^{-1}\right),
$$

as readily shown by a simple calculation. Therefore, this equation and the second equation above lead us to

$$
\frac{q\left(R^{-1} \mid x_{2}\right)}{q\left(R \mid x_{1}\right)}=R \frac{p_{1}\left(x_{1}\right)}{p_{1}\left(x_{2}\right)} .
$$

Note that this is a consequence from detailed balance alone.

With the additional assumption of Gibrat's law, one can immediately rewrite this equation as

$$
\frac{p_{1}\left(x_{1}\right)}{p_{1}\left(x_{2}\right)}=\frac{1}{R} \frac{q\left(R^{-1}\right)}{q(R)} .
$$

Note that the left-hand side is a function of $x_{1}$ and $x_{2}=R x_{1}$, while the right-hand side is a function of only $R$. The equality holds if and only if $p_{1}$ is a power-law function. (For example, if we expand this equation in terms of $R$ around $R=1$, we obtain a differential equation that $p_{1}$ has to satisfy, one whose solution is the power-law and is verified as satisfying the above equation.)

It is also possible to obtain a proof by inserting the power-law function $p_{1}(x) \propto$ $x^{-\mu-1}$ into the above equation so that the reflection law follows as a consequence. 


\subsubsection{Copula}

Gibrat's law, detailed balance, and so forth have been uncovered by conducting an analysis of the statistical dependence between multiple variables. A powerful method beyond conventional approaches can be applied to such an analysis, and more generally to other analyses regarding correlations, namely the copula method.

For two stochastic variables a copula is a function which represents the joint probability function of the variables in terms of cumulative probability functions for each of the variables. A cumulative probability function $P_{<}(x)$ for a stochastic variable is defined, as explained on page 21 , as the probability that the variable takes a value smaller than $x$. For two stochastic variables the joint cumulative probability function $P_{<}(x, y)$ is the probability that the first variable is smaller than a value $x$ and that the second one is smaller than $y$. If the two stochastic variables are statistically independent of each other (you cannot predict one from the other), then the probability is simply the multiple of each variable's cumulative probabilities:

$$
P_{<}(x, y)=P_{<}(x) P_{<}(y)
$$

This is not always true, however. Yet one is able to show that the joint probability can be expressed by a function of each variables' cumulative probabilities as

$$
P_{<}(x, y)=C\left(P_{<}(x), P_{<}(y)\right) .
$$

The function $C(u, v)$ that appears here is the copula (more mathematicallyoriented readers may consult Nelsen (2006)). ${ }^{13}$

The word "copula" is used in linguistics as the connecting link between the subject and predicate of a proposition. In statistics, it refers to a link or bond which connects two stochastic variables, and to the method involved in joining them.

Since the copula function has variables consisting of cumulative probabilities $P_{<}(x)$, it is a multi-variate function with the domain of its argument variables being between 0 and 1 . In addition, a copula represents a joint probability, so the value it takes ranges from 0 and 1 . Note that copula are invariant under an appropriate change of scale in the stochastic variables, because the argument variables are cumulative probabilities, but not stochastic variables. If the stochastic variable is money, we have the same copula irrespectively of how the variable is represented in units of dollars, millions of dollars, or the logarithm of a dollar sum. We can even go one-step further, and use only the rank (smaller or larger), instead of the value of $x$. In this sense, therefore, copula is the concept regarding so-called order-statistics. Remember that the concept of correlation coefficient (see the mathematical column on page 121), and related concepts, are based on the realm of normal

\footnotetext{
${ }^{13}$ It is possible to generalise this to more than two stochastic variables, though we shall focus on pairs of variables.
} 

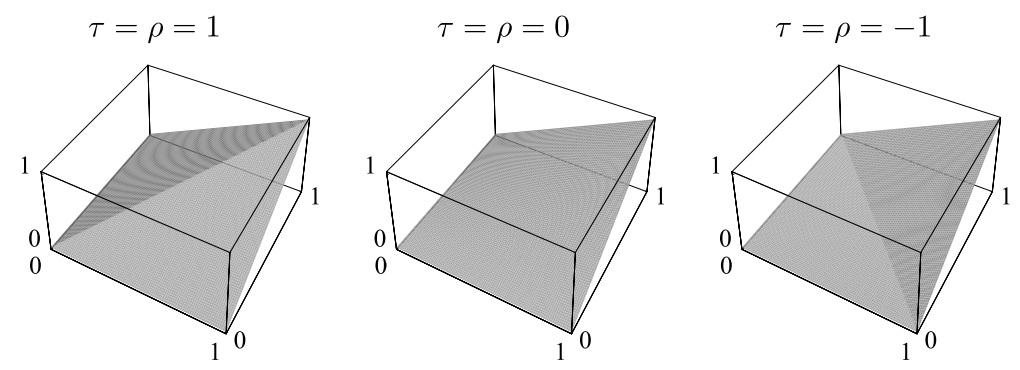

Figure 3.9: Three typical examples of copula.

distributions. ${ }^{14}$ In contrast, copula have desirable properties which enable us to describe distributions with fat tails, such as the Pareto distribution, since it does not depend on assumptions for each stochastic variable's distribution (marginal distribution). Copula can also be used to conduct simulations in multi-variate statistical problems that involve statistical correlations, for example in quantitative finance, risk management, and so forth (see Iyetomi et al. (2009b) for example).

Interestingly, the copula function $C(u, v)$ has an upper-bound function and a lower-bound function, as shown in Figure 3.9. In each of these figures, the two axes on the horizontal plane are $u$ and $v$, the ordinate is the value of $C(u, v)$. The left figure gives the upper-bound, while the right illustrates the lower-bound, and the middle figure depicts the case of two statistically independent variables. $\tau$ and $\rho$ in these figures are to be understood as follows. Kendall's $\tau$ is a parameter characterising the copula, it takes a value from -1 to 1 correspondingly to the lower and upper bound of the copula, while it is exactly 0 in the case of statistical independence. ${ }^{15}$ Let $P_{+}$be the probability that the relative ordering of the ranks with respect to $x$ is the same as the relative ordering of the ranks with respect to $y$ (so-called concordant case); and let $P_{-}$be the probability that the relative ordering of the ranks with respect to $x$ is opposite to the relative ordering of the ranks with respect to $y$ (i.e. it is a discordant case). Then assuming that there exists no tie or similar rank in either of $x$ or $y$, we have

$$
\tau=P_{+}-P_{-} \simeq 2 P_{+}-1
$$

which reads as the meaning of $\tau$. Suppose, for example, that the variables are company-sizes in two consecutive years. If you choose two companies at random, the relative ranking of the two companies in the first year is always

\footnotetext{
${ }^{14}$ One can appreciate this simply by noting that a proper definition of correlation coefficient requires a finite variance, but in fact our world has enough space for distributions without finite variances.

${ }^{15}$ This parameter is also called Kendall's rank correlation $\tau$.
} 


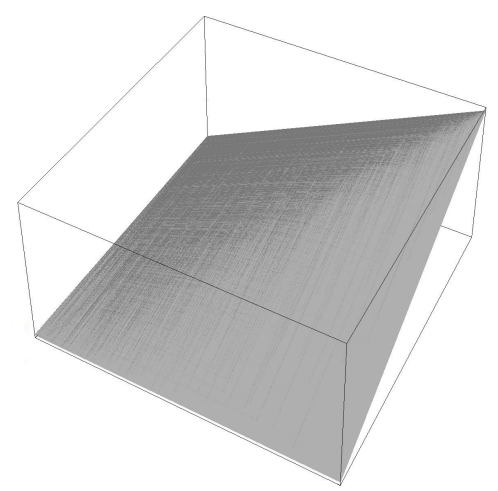

Figure 3.10: Copula for company-incomes in the years, 2001 and 2002 .

the same as the relative ranking in the second year, if $\tau=1$. And it is fiftyfifty, if $\tau=0$. If $\tau=-1$ then the rankings are always opposite to each other,

The parameter $\rho$ is conventionally known as Spearman's $\rho$. This concerns a triplet of companies rather than a pair. Let us be satisfied here with understanding this as (a linear function of) the volume under the surface spanned by the copula function. It is -1 for the lower-bound, 1 for the upper-bound, and 0 in the case of statistical independence.

We will now turn to examine examples of copula for actual data.

Figure 3.10 depicts the copula for Japanese company incomes in the years 2001 and 2002, as studied in Section 3.1.2. The copula has similarity in its functional shape as the upper-bound function shown in Figure 3.9. The parameters of $\tau$ and $\rho$, actually derived from the real data, are tabulated in Table 3.1. The value of $\tau$ implies that for a randomly chosen pair of companies the probability that the relative ordering of the ranks in the first year is the same as that in the second year is approximately $80 \%$.

The various data for European countries have similar copulas. Table 3.1 exemplifies this point from the total-asset data for French companies.

For Japanese personal income data the copula has a qualitatively different function from the upper-bound. In fact, the parameter $\tau$ is nearly 0.6 , as listed in Table 3.1, which means that for a randomly chosen pair of highincome earners the probability that the relative ordering of the ranks in the first year is opposite from that in the second year is roughly $20 \%$. Some readers will be relieved to hear that this turnover probability does not vanish to zero.

We are also able to test Gibrat's law by using copulas. In the preceding section, the validity of the law was clear from the PDF figures, but it is 
Table 3.1: Parameters of $\tau$ and $\rho$ for copulas

\begin{tabular}{|c|c|c|}
\hline data & $\tau$ & $\rho$ \\
\hline \hline Tax-income (Japan): 2001-2002 & 0.59 & 0.77 \\
\hline Total-assets (France): 2000-2001 & 0.86 & 0.95 \\
\hline Personal-income (Japan): 1997-1998 & 0.62 & 0.72 \\
\hline \hline Tax-income and its growth-rate (Japan): 2001-2002 & 0.02 & 0.05 \\
\hline Total-assets and its growth-rate (France): 2000-2001 & 0.03 & 0.04 \\
\hline Personal income and its growth-rate (Japan): 1997-1998 & -0.04 & -0.05 \\
\hline
\end{tabular}

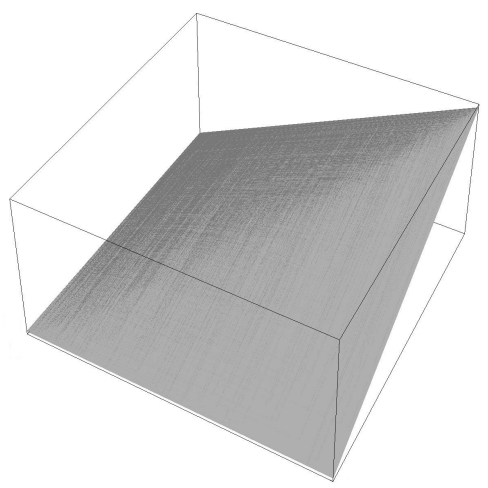

Figure 3.11: Copula for personal-incomes in the years, 1997 and 1998. 


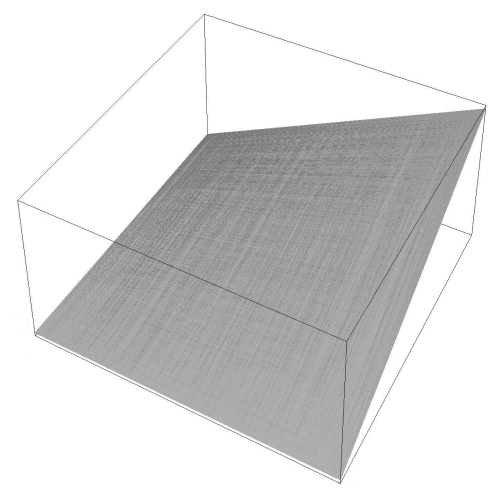

Figure 3.12: Copula for company-incomes in the year 2001 and its growth-rates.

valuable to quantify the validity in terms of a copula and its parameters.

Figure 3.12 shows the copula for the tax-income of companies in 2001, and its growth-rate from that year to 2002. It can be observed that the copula function is close to the middle copula of Figure 3.9. Actually, both parameters, $\tau$ and $\rho$, are close to zero. These facts ${ }^{16}$ show that Gibrat's law holds with a respectable accuracy, as quantified by the copula and its parameters. Similarly for total-asset data of French companies. ${ }^{17}$

In addition, for personal income and its growth-rate, the parameters $\tau$ and $\rho$ have small absolute values, as shown in Table 3.1. (The copula figure is omitted here.) Thus we can have further evidence for the validity of Gibrat's law.

It should be remarked that the copula method will shortly be shown to open the way to a more sophisticated method of performing simulations of multi-variate problems.

\subsection{Digression: Personal-income Fluctuation}

\subsubsection{Gibrat's Law and Detailed Balance}

Let us make a brief digression away from company growth, and instead consider personal income in relation to Gibrat's law and detailed balance. In doing so, we will show that Gibrat's law and detailed balance, as well as the Pareto's law, can break down, depending on the economic environment (see Fujiwara et al. (2003)). We focus, as we have done hitherto, on the highincome region where Pareto's law holds.

${ }^{16}$ Calculated for the top ten thousand companies in the year 2001.

${ }^{17}$ Calculated for the top two thousand companies in this case. 


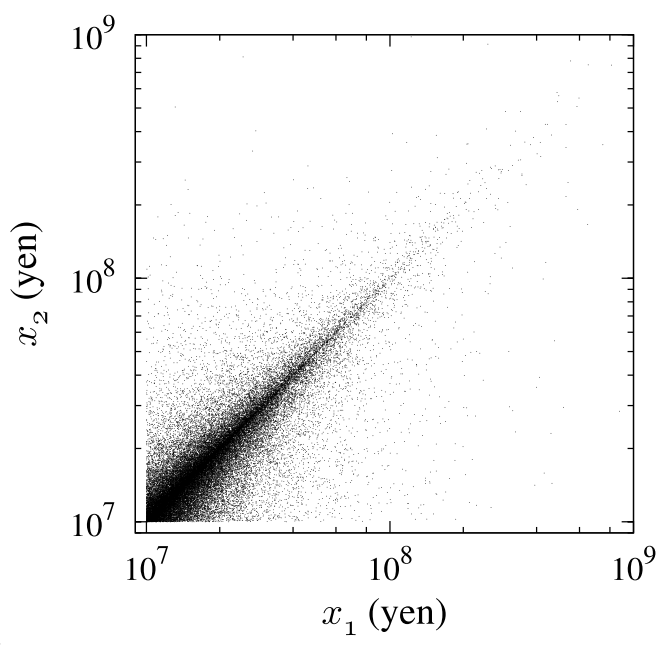

Figure 3.13: Scatter-plot for personal-incomes (measured by the amount of taxes paid) for two consecutive years (1997 and 1998).

Consider on the one hand, the process by which a person's annual income of 20 million yen doubles in the following year, and on the other, the doubling process for an initial income of 40 million yen. Do they differ, and if so, how? Alternatively, take the probability of these incomes halving in the following year. At the risk of offending our readers, those with moderate incomes may wrongly guess that fluctuations of this scale are negligible for high income earners.

We will perform the same analysis on the study of fluctuation of personal income as we did for company growth. Let the last year's annual income be $x_{1}$, and this year's $x_{2}$. We can calculate from the dataset of high-tax payers each person's growth-rate $R$. How is this possible? From the Japanese datasets in the fiscal years 1997 and 1998, for example, we can identify high-tax paying individuals, and make a list of those persons appearing in both years' data. (See Section 3.2.3 if you are interested in the story behind this exhaustive Japanese data set).

We first show in Figure 3.13 the scatter-plot for income-tax pairs $\left(x_{1}, x_{2}\right)$ for these high-tax payers (some 52 thousand extracted from 93 thousand taxpayers in the fiscal year 1997, and from 84 thousand in 1998). The values of $x_{1}$ and $x_{2}$ respectively represent the income-taxes in 1997 and 1998 (both in yen). The lower limit in the data is 10 million yen, so we are left with the points in the rectangular region $x_{1}, x_{2}>10^{7}$. In passing, it should be noted that there are a few extremely high-tax payers towards the north-east, beyond the scope of the figure. 


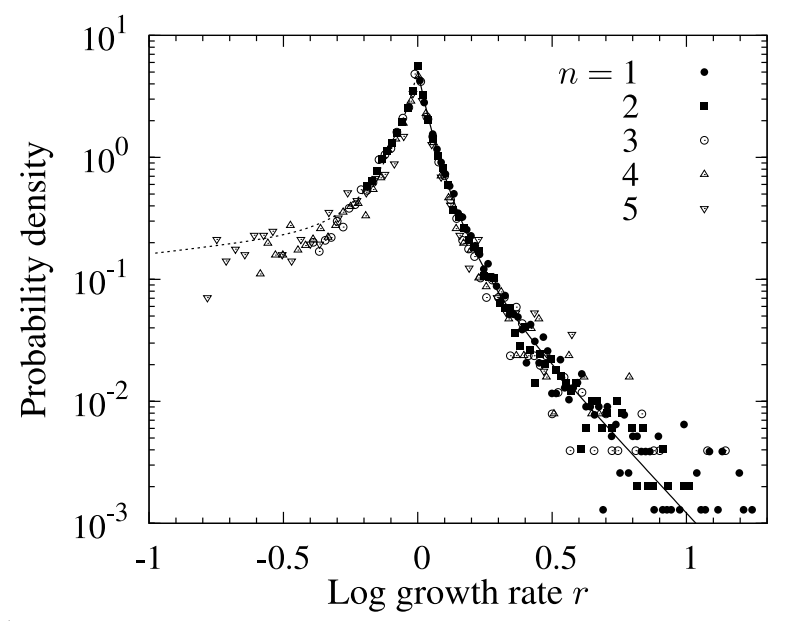

Figure 3.14: Probability distribution for growth-rate of personalincome (1997 to 1998).

A statistical test for detailed balance gives no ground for the rejection of the null hypothesis that the joint probability distribution is symmetric, so the data is compatible with the property of detailed balance.

Then we proceed to calculate the PDF as a histogram for the logarithmic growth-rate $r=\log _{10} R$ conditioned on the value of $x_{1}$, and obtain the result in Figure 3.14. Here the bins $n=1, \ldots, 5$ are used corresponding to five logarithmically equal intervals from 10 million $\left(x_{1}=10^{7}\right)$ to 100 million yen $\left(x_{1}=10^{8}\right)$. Because numerically $10^{0.2} \simeq 1.58$, these bins are sequentially multiples by 1.58 up to the limit of 10 million yen.

It is clear from the figure that the distribution for the growth-rate collapses into a single curve irrespective of the bins considered, indicating that the growth-rate is statistically independent of the previous year's income-tax (Gibrat's law). In other words, for the high-tax payers present in the Pareto region, the transition probability for a doubling or halving of income is independent on initial income.

We additionally see in Figure 3.14 that the calculated function of the PDF in the side of $r<0$ by the reflection law from the nonlinear fit in the side of $r>0$ yields a satisfactory fit.

We conclude, therefore, that both Gibrat's law and detailed balance hold for personal income, as we conjectured on page 86 . 


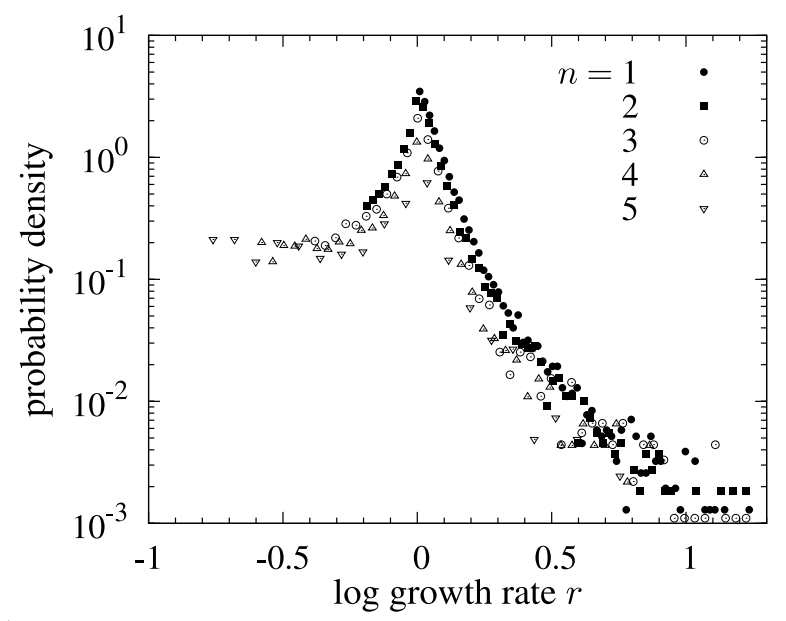

Figure 3.15: Probability distribution for growth-rate of personalincome ,1991 to 1992, corresponding to the bubble collapse in Japan.

\subsubsection{Breakdown of the Laws}

By taking a close look at the distribution for the growth-rate of personal income in Figure 3.14, we can see that there is a cusp at $r=0$ implying little variation in income, so relatively stable income-earners are dominant. On the other hand, there is a non-negligible percentage of people whose income grows or shrinks by large magnitudes. While the Japanese Tax Law did not compel the high-tax payers to reveal the sources of their incomes, it is reasonable to assume that they were derived from financial assets and real estate, an assumption that can be verified by Japanese government surveys on Family Income and Expenditure.

If such high risk assets as real estate and stocks are prone to large variations in their returns, personal income would be obviously be sensitive to such variations, particularly for those with high-incomes. And, when a macroscopic shock occurs, the laws and balance so far observed can break down, as we shall see shortly.

In the late 1980s, Japan experienced an abnormal rise in the price of real estate and stocks peaking during 1990 and 1991, followed by a great plunge in the markets. The period is colloquially known in Japan as the Bubble, or the Heisei bubble, the name being derived from the term used to describe the reign of the emperor. A number of high-income earners rose into the Pareto region just before 1991, and after that year, the majority of them disappeared. Hitherto stable high-income earners were also subject to a great variation in their risky assets. Examination of the details for two years' of 
data for high-tax payers shows that:

- Pareto's law broke down, and the distribution deviated from a powerlaw,

- Gibrat's law also broke down as shown in Figure 3.15, and

- Detailed balance did not hold.

That is to say, while the Laws and Balance hold under relatively stable circumstances, they can break down in the presence of drastic changes in the macro-economic environment (see also Aoyama et al. (2004)).

This observation completes our description of the phenomenology of fluctuation.

\subsubsection{Side-story: Public Notice of High-Tax Payers, and Lost Data in Japan}

From 1947 to 2005, Japan required a legal public notice of income, in accord with the Income Tax Law, the initial purpose of which was "to reduce tax evasion through public notice of high-tax payers' incomes, allowing them to be checked by independent agents". (Later, publication was justified on the grounds that it gave publicity to those making large contributions to society.) Indeed, for a while after taking effect, information providers who revealed tax dodgers were offered rewards depending on the amount involved in tax evasion. The Income Tax Law, Article 233, required that income earners "give notice to the public of the names, addresses, and amount of income-tax of the tax-payers whose income-tax exceeds 10 million yen", and also the Ordinance of Enforcement 106 in the Tax Law states that "the relevant district director must post the notice by displaying it at the posting area in the district or at a public area within the district".

This law, which is presumably quite unusual in the world context, can potentially infringe privacy, or assist criminals in finding targets, since the names, addresses and the tax amounts have posted every May in front of tax offices all over the country. After considerable dispute the law was abandoned in 2006, partly due to the weakening of the initial purpose, the prevention of tax evasion, as well as the newly issued Privacy Law.

While the problems of privacy encroachment and criminal risk were clear and hardly debatable, the data is, nevertheless, of remarkable intellectual value. Without it, it would not have been possible to reveal the personalincome distribution for the entire range of incomes or conduct an investigation of individual growth-rate. Indeed, Japanese economists have benefited significantly from the existence of this and other datasets concerning socio-economic inequality, and when presenting the results at international conferences we have observed that colleagues were as surprised by the existence of the data as they were by the results. 
Indeed, in the high income region the tax data is exhaustive, which is not the case in most datasets examined since Pareto. For example, the wellknown economist D. G. Champernowne was advised by J. M. Keynes to study personal-income for his thesis, and proposed a stochastic model to explain the distribution, which describes transition of an individual's income from one moment to another, or growth process (see Champernowne (1973) for details; and also Mandelbrot (1997, chap. E11)). However, to the best of our knowledge, there has been no work that directly observes the process, and such an investigation has been regarded as a formidable task up until the present time.

The Pareto distribution and its index, the distribution of growth-rate, are matters of interest not only for economists examining the skewed distribution of high-income earners and the origin of this phenomenon in terms of growth, but are also of significance for tax administrators aiming to maintain government tax revenue and manage the associated risk.

Furthermore, it should be remarked that the data serves as a tool for measuring "social disparity", since the tail of the Pareto region and its quantification is complementary to the well-known Gini coefficient described in Section 2.4.1. Let us recall the way in which the long tail of the distribution is stretched towards the high-income side. If we take readily available data published by the Japanese National Tax Authority in Japan, as with the tabulated data in other developed countries, we find a single point for the total number of high-income earners (above a few tens of millions of yen in the Japanese case).

One of the present authors (Y. F.) raised this issue with a member of the Japanese Government's Tax Commission. Since the abolition of the law requiring the publication of tax data this material is no longer available, and while personal privacy is a critical concern, it should not become an excuse for ceasing to think about the issue. Perhaps the government could have improved the law instead of abandoning it. We can agree that the precise relation between a particular tax-payer and the certain amount of incometax is merely intrusive gossip, but it might be argued that publication and scientific analysis of the stretched long tail of personal-income distribution and its changes over time is in the public interest. It would be interesting to know how readers react to this matter.

\subsection{Small and Medium-sized Companies}

\subsubsection{Large-scale Data for Small and Medium Enter- prises}

We have so far focused only on bigger companies in Japan and in Europe, and as explained in the preceding section, the Pareto region is dominated by a few giant companies comprising a large part of the aggregated quantities of size, so the dynamics of these firms are obviously important. However, of equal 


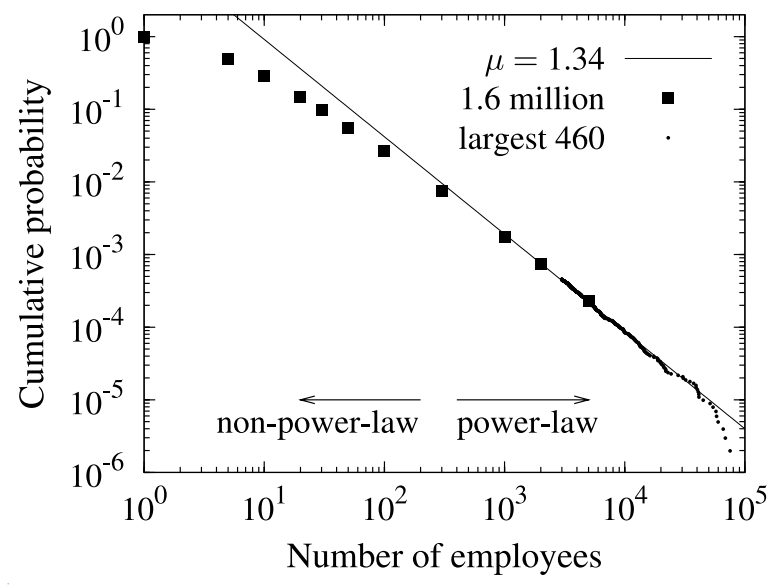

Figure 3.16: Cumulative distribution for company-size measured by number of employees (whole range; year 2001).

importance are the many dwarfs, the small and medium enterprises, which form the majority of companies.

One of the primary concerns of financial institutions is to determine a reasonable interest rate in financing a large number of small and mediumsized companies. The provision of a quantitative and statistical evaluation of financial risks for such a number of financial entities requires the public availability of a large-scale dataset covering those companies and firms in exhaustive detail.

The Credit Risk Database (CRD) for Japan was founded in 2001 as a membership organization to collect financial and non-financial data, including default information, on "small and medium enterprises" (SMEs). In 2005 it was renamed the CRD Association. The purpose of establishing CRD was to promote the streamlining and efficiency of SME financing by assessing their business conditions on the basis of data gathered by measuring credit risks related to SME financing. As the membership and data collection expanded, the CRD established its footing as an infrastructural basis for the gathering and provision of SME data. This data is provided by commercial banks, government-affiliated financial institutions, and the National Federation of Credit Guarantee Corporations, which collects information from more than a million SMEs across the country.

For the purposes of our discussion in this text we accept the definition of SMEs as laid out in the Small and Medium Enterprise Basic Law. If the company's stated capital or number of employees is less than a threshold specified by the law, the company is said to be an SME, the exact threshold depending on the business sector to which the company belongs. For the 
manufacturing sector, companies with less than 300 million yen of capital or 300 employees are SMEs. For wholesale businesses the level is set at 100 million yen of capital or 100 employees, while for retailers the threshold is 50 million yen or 50 employees. Service industries with less than 50 million yen or 100 employees are classified as SMEs.

Figure 3.16 depicts the cumulative distribution for company-size (number of employees) in the whole range where data are available based on the Establishment and Enterprise Census (Ministry of Internal Affairs and Communications) in the fiscal year of 2001, and also on an exhaustive list of the largest 460 companies (provided by a credit research agency). The tail of the distribution will by now be familiar, since it is the Pareto region where a power-law applies. A deviation from the power-law can be observed in the smaller region, with a qualitative change occurring round about the 200 to 300 employee point. The CRD, therefore, covers the non-power-law region as well as the Pareto region, irrespective, for the most part, of the business sector, on which the criterion in the data is dependent. This is ideal for the following study.

\subsubsection{Size-Dependence of Growth}

At this point we turn to examine the growth-rate and distribution for the stock variables of total-assets and debt, and for the flow variable of sales. We can expect the character of fluctuation in the non-power-law region and its transition to the power-law, to differ from that considered in Section 3.1 where we examined the power-law regime, and our purpose in this chapter is to demonstrate that difference (see Fujiwara et al. (2006a,b) for more details).

We will create eight bins, in the same way that we did for large companies in Section 3.1.1, divided by logarithmically equal intervals from 10 million to 1 billion yen for each of total-assets, debt and sales. We can then calculate the PDFs for the logarithmic growth-rate $r$ conditioned by the size of each corresponding bin. The result is shown in Figure 3.17.

Immediately we can see that Gibrat's law does not hold, as it is clearly evident that for each of the PDFs the distribution for the growth-rate differs depending on the bin or the size. Noting that the indices $n=1, \ldots, 8$ are numbered from small-sizes to large-sizes, the PDF's show that the typical variation in the growth-rate's distribution becomes smaller, as is plain for the cases of larger companies.

To quantify this fact, we can calculate the variance $\sigma$ of the growth-rate distribution, which reveals that the initial size $x_{1}$ is the controlling variable, as shown in Figure 3.18. This dependence can be expressed quantitatively as a scaling relation:

$$
\sigma \text { is proportional to } x_{1}^{-\beta} \text {. }
$$

Here $\beta$ is a constant whose value is close to 0.5 .

It appears that there is a threshold below which the variance in the growthrate decreases for the larger size of companies, and above which the growth 

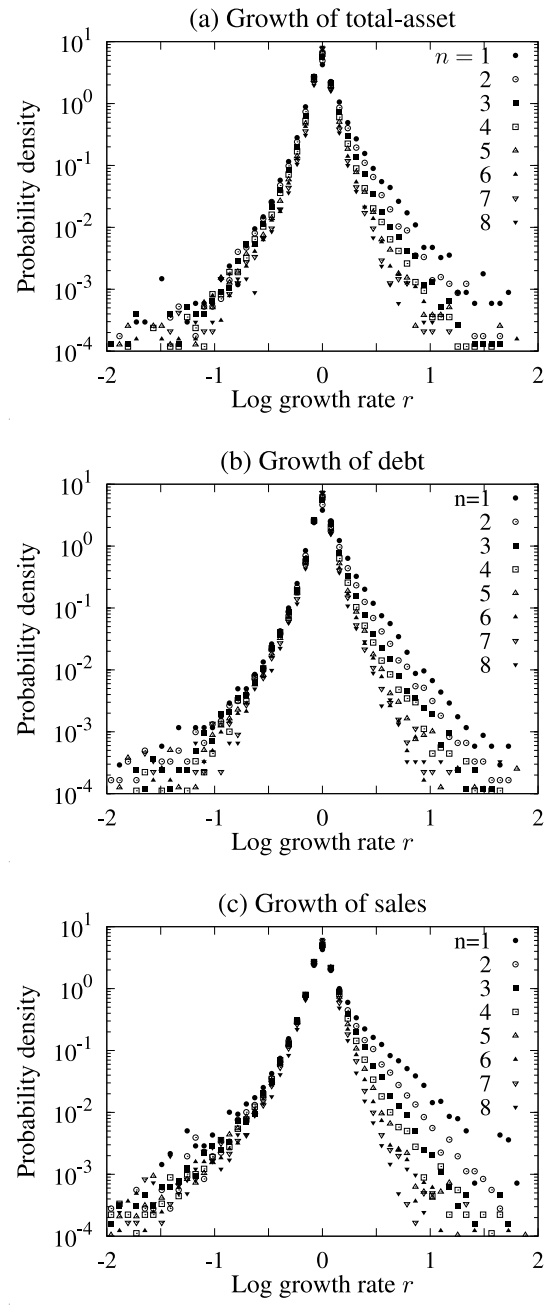

Figure 3.17: Probability distribution for growth-rates of small and medium companies: ((a) total-assets, (b) debt and (c) sales; years $2001 / 2000)$. 

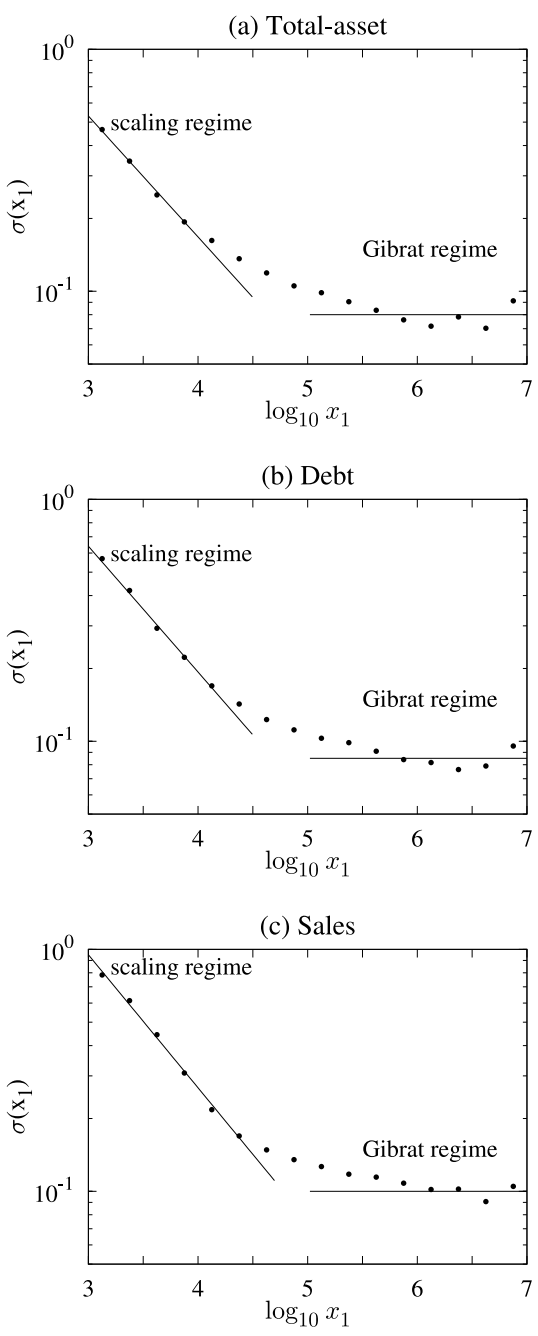

Figure 3.18: Relation between company-size and variance of growth-rate for small and medium companies: ((a) total-assets, (b) debt and (c) sales; years 2001/2000). 
rate does not change, and Gibrat's law holds. Moreover, the threshold marking the transition of these two phases may differ depending on the variables chosen, i.e. total-assets, debt, sales etc.

In summary, we observe from our study of the growth-rate of companies and firms that:

- There is a size below which Gibrat's law does not hold, implying that there is a "natural kind" of "small and medium-size companies", as opposed to a merely legal definition,

- The variance in the distribution for the growth-rate of small and mediumsized companies has a scaling relation with respect to the company-size, and the scaling relation is of real significance in a quantitative analysis of credit risk management.

\subsection{Companies Bankruptcy}

\subsubsection{Companies Activity and Bankruptcy}

We so far have talked mainly about the growth of companies, but in fact the activities of companies have, in the abstract, three facets: (1) obtaining finance from financial institutions, markets and investors, and (2) investing money to add value to materials and services in anticipation of future return of profit, and (3) collecting the realised profit from the sales minus costs.

The flip sides of these activities implying, however, (1) the presence of creditors other than the company's investors, (2) the risk of the loss of invested money, because (3) the profit is necessarily uncertain.

The increase or decrease of stock variables or growth in a company's activities is associated with two aspects of money flow, namely, where the money comes from and goes to, and how it does so.

The idea of double-entry bookkeeping is to describe these aspects of a company's activity (see Pratt (2008) for example). As is well-known, the balance sheet $(\mathrm{B} / \mathrm{S})$ describes stock variables, while the profit and loss $(\mathrm{P} / \mathrm{L})$ account describes flow variables. ${ }^{18}$ The left-hand side (the debit column) records the state of money in terms of where it goes to and how it is used, while the right-hand side (the credit column) describes the cause of an increase or a decrease of money in terms of where it comes from and how it is financed.

The basic ingredients in a $\mathrm{B} / \mathrm{S}$ are

- assets (left): funds in a business which are considered likely to bring about a future increase of the company's fund,

- liabilities (right): debts which provide a temporary increase of funds at a moment but will cause a future decrease of the fund when it is returned to the creditors,

\footnotetext{
${ }^{18}$ An additional book describes cash-flow, which enables the observer to understand the causes of increase and decrease in a company's money.
} 
- capital (right): assets minus liabilities, by definition, which is the share by the company's stockholders. This consists of the stockholders' investment and retained earnings arising from the flow of profit.

The ingredients in the $\mathrm{P} / \mathrm{L}$ are

- costs (left): money used to gain profit, including the purchase of intermediate materials and services, labour costs, and various other costs,

- profit (right): revenue minus costs,

- revenue (right): increase of the company's money in consideration of value added that was generated in the activity of production.

A change in the stock is equal to the flow, and usually speaking, profit increases the capital as retained earnings. Also, additional investment by stockholders increases the capital. On the other hand, borrowing from financial institutions and markets, such as bonds, increase the liabilities or debts.

Suppose that the company has negative capital, that is the state of excessive debts over assets, then the company will have difficulty in returning the money borrowed from creditors. This is the state of negative net worth. A flow of profit can bring about the possible resolution of this critical state, but more probably the company will be plagued with serious funding problems and run out of capital.

Bankruptcy is a general term referring to the process in which a company falls into the state of financial insolvency as a debtor. It is classified by legal and private procedures, namely, suspension of bank transactions, corporate reorganization and rehabilitation, procedure for liquidation, etc. Specifically, in Japan, two note payment defaults made by a company within six months forces all transactions of the company to be suspended. More generally, a bankruptcy refers to the malfunction of a company's activities due to critical problem of funding in one form or another.

\subsubsection{Debt when Bankrupted and Life-time}

In Section 5.3, we will describe the ways in which one can model the dynamics of a balance sheet, but at this point we will pause to examine the phenomenology of the process of bankruptcy, using exhaustive Japanese data (see Fujiwara (2004) for more details).

Over the last 10 years, the annual number of bankruptcies has varied from 10,000 to 20,000, as shown in Figure 3.19 by a dotted line (see the right axis), while the total number of companies in Japan is, at a rough estimate, approximately two million. ${ }^{19}$ Bankruptcy statistics are obtained from Small

\footnotetext{
${ }^{19}$ Figures according to the National Tax Agency's Statistical Survey. There are two other major sources of statistics, the Ministry of Internal Affairs and Communication's "Establishment and Enterprise Census", and the Ministry of Justice's records on the entry and exit of companies. These statistics are said to have under- or over-estimation problems arising from, for example, the counting of non-active companies etc.
} 


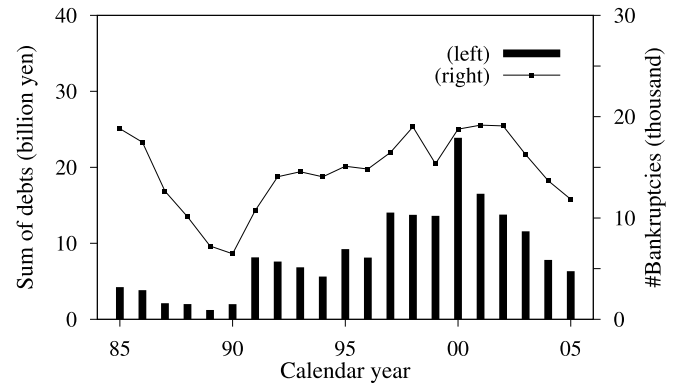

Figure 3.19: Annual number of bankruptcies in Japan and total sum of resulting debts (1985 to 2005; calendar-years).

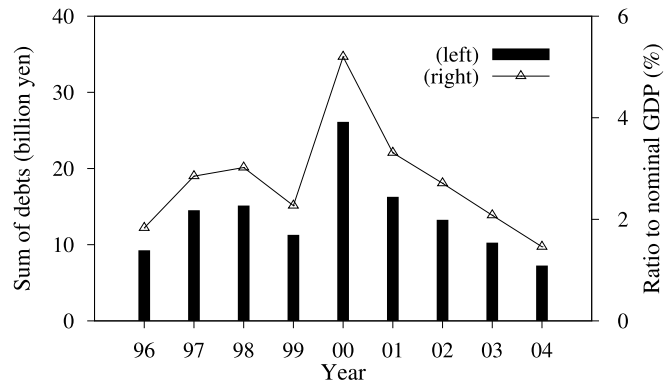

Figure 3.20: Annual sum of debts when bankrupted, and ratio to nominal GDP (1996 to 2004; fiscal years). 


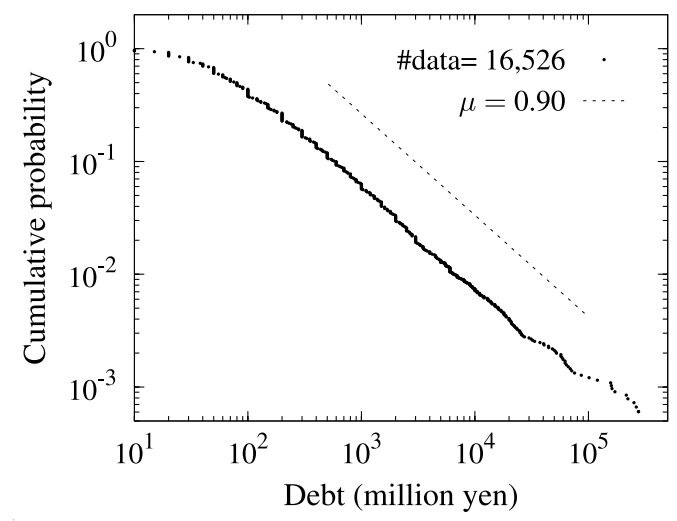

Figure 3.21: Cumulative distribution for debt when bankrupted (approximately 16,000 companies bankrupted with debts larger than 10 million yen in 1997).

\& Medium Enterprises and Regional Innovation in Japan (SMRJ). The figure plots bars representing the total debts of bankrupted companies across the country, varying from 10 trillion to 25 trillion yen, as shown in the left axis (see also Fujiwara (2008)).

In the data accumulating the total debts in fiscal years, the quantity of debts involved in each year's bankruptcy is more than $2 \%$ of the nominal GDP in the year, as can be seen in Figure 3.20 (line; right axis). Of course, not all the debts are lost, since some are partially returned to creditors, who are eligible to receive a portion of the credit from the bankrupted debtor with a ratio determined by the types of bankruptcy and how the creditors are situated. It should, however, be noted that a very large sum of money is at risk every year in Japan; there is no reason to suppose that this is not the case in other countries too.

This point deserves serious consideration, since the distribution for companysize when bankrupted, and accordingly that for the amount of debt, has a heavy tail, implying that a giant bankruptcy can occur at a non-negligible probability. Indeed, with the help of an exhaustive list of bankruptcies with debts of 10 million yen or more in the year 1997, essentially covering all the domestic bankruptcies, the distribution for the debt of 16 thousand bankrupted companies can be depicted in Figure 3.21.

To assist the naked eye, a dotted line corresponding to the Pareto distribution with the index $\mu=0.9$ is drawn on the figure. The result shows that in the wide range of debt larger than 500 million yen, debt when bankrupted obeys Pareto's law for the tail of the distribution. Moreover, for those large bankruptcies, we can calculate the ratio $A=d / s$ between the sales $s$ and the debt $d$ immediately before the bankruptcy. Interestingly, the distribution 


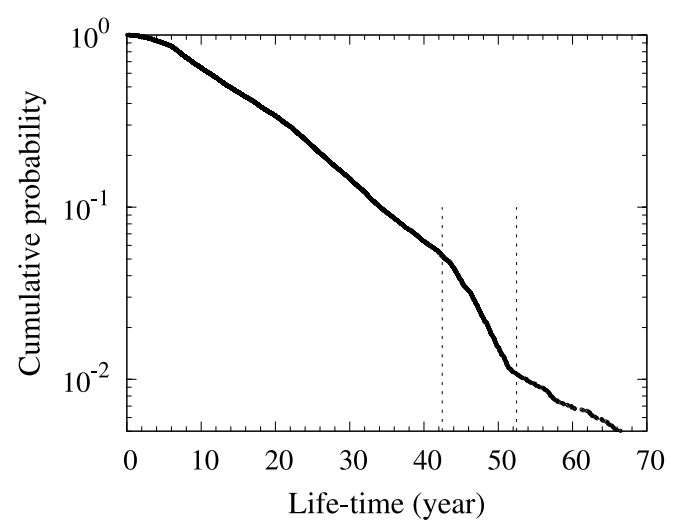

Figure 3.22: Cumulative distribution for life-time before bankruptcy (approximately 16,000 companies bankrupted with debts larger than 10 million yen in 1997).

$p(A)$ is statistically independent of the size $s$. It follows from this observation that the distribution $p(s)$ for company-size $s$ and the distribution $p(d)$ for its debt $d$ are compatible with each other, and obey the Pareto distribution with approximately the same Pareto index. Pareto's laws for company-size and for debt when bankrupted are consistent in this sense.

We can also examine the lifetime of company before bankruptcy, by studying the time elapsing between establishment of the company to its financial collapse. Figure 3.22 depicts the cumulative distribution for the lifetime, and shows that it is an approximately exponential distribution. Note that the abscissa in the figure is linear. If we were to estimate the typical time before company death from the age at the cumulative probability of $10 \%$ or $10^{-1}$, we would arrive at the figure of roughly 30 years. Incidentally, if we look back through the 45 year era before 1997, the time-scale involved in the survival process of companies changes drastically within 10 years. This corresponds to the dissolution of the corporate-alliances monopoly, a measure enacted during the government of US General Headquarters (from 1945 to 1952), during which a large number of new companies entered the market, as confirmed by mathematical argument.

\subsection{Production Function and Ridge Theory}

\subsubsection{Production Function}

The activities of companies are primarily aimed at earning profits from sales, after spending and investing money on inputs of labour and capital. How do 
companies determine the amounts of inputs to invest?

Suppose that a company can make an amount of production $Y$ from certain amounts of capital $K$ and labour $L$. The relation between the inputs and the output is the production function. (See Varian (2005) for example).

As early as in the spring of 1928, the economist P.H. Douglas had analysed a plot of data for capital, labour and production, and got into difficulties. Seeking for a reasonable function to fit the data, he discussed with his friend the mathematician, C. W. Cobb, and, together they proposed a function of this form:

$$
Y=F(L, K)=A K^{\alpha} L^{\beta}
$$

which is today known as the Cobb-Douglas production function Cobb and Douglas (1928). Here $A$ is a constant of proportionality, and $\alpha$ and $\beta$ are the selectable parameters. In the special case for which $\alpha+\beta=1$, the production function can be written as

$$
F(L, K)=A L\left(\frac{K}{L}\right)^{\alpha}=L f(x), x \equiv \frac{K}{L} .
$$

This states that the production function is an extensive quantity, to use the terminology of statistical mechanics. That is to say that if a physical system can be analysed by thermodynamics and statistical mechanics then it is possible to calculate its macroscopic state variables. If these state variables do not depend on the system's size they are called intensive, while those depending on the size are called extensive. Examples of intensive quantities include pressure $P$, and temperature $T$, while the extensive quantities are internal energy $U$, volume of the system $V$, entropy $S$, and so forth (see Callen (1985) for example).

Now, managers in companies make decisions on allocating inputs between labour and capital; they could, for instance, purchase new machines in factories, and at the same time, reduce employment. The intention in such a case is to increase efficiency in production by augmenting the reduction of labour with the increase of capital. Economists often express the allocation between capital and labour as a "substitution between capital and labour". The measure used to quantify the extent of this activity is the elasticity of substitution denoted by $\sigma$. In the case of the Cobb-Douglas production function, $\sigma=1$. In fact, there are different views on the substitution between capital and labour. For example, the economist W. Leontief, claimed that there is no substitution between them in company production in Leontief (1941). This claim amounts to saying that the level of production is determined by the minimum of capital or labour. This corresponds to $\sigma=0$, which is quite the contrary to the Cobb-Douglas case.

These views might be regarded as too extreme, but in fact, in 1961, the economist K. J. Arrow, with his collaborators, interpolated both extremes of Cobb-Douglas and Leontief, and proposed a production function, called CES (constant elasticity of substitution) (Arrow et al., 1961). In this case $\sigma=0 \sim 1$, and the CES production function includes a special case of perfect 
substitutes, which is expressed by

$$
F(L, K)=A\{\delta L+(1-\delta) K\},
$$

where $\delta$ is a constant. The set of all possible combinations of capital and labour which are sufficient to produce a given amount of output are simply a linear combination of $K$ and $L$.

Yet another new proposal for the production function followed in the literature, suggesting that the elasticity of substitution is a constant for all the cases of Cobb-Douglas, Leontief, and CES. Generalisation of this is clearly possible to allow elasticity, and is given by some function of capital and labour. A well-known example for this kind of production function is the one proposed by L. R. Christensen et al. in 1973 (Christensen et al., 1973), under the name of the transcendental logarithmic production function.

There are still other types than those we have seen so far, but with a variety in the number of parameters included in the definition of production function. There is as yet no decisive answer as to which are best, partly because it is trivial to achieve a better fit to data with larger number of parameters, though one could attempt to obtain an answer by employing some sophisticated technique developed in statistics.

For example, if we take data for about a thousand companies listed in the first sector of Tokyo Stock Exchange in 2004, we can find that the CobbDouglas production makes a relatively better fit than others, with the resulting parameters satisfying $\alpha+\beta=1.02$, which implies the extensive nature of the production function. For the same data set we find that $\alpha=0.121$ and $\log A=$ 0.081 , so for our purpose here, we content ourselves by observing the validity of the extensive claim, and we will adopt the Cobb-Douglas production function in what follows.

\subsubsection{Ridge Theory for Companies Growth}

The problem now facing us is to explain the behaviour of the population of companies through their attempts to maximise profits. In addressing this matter we shall focus on production function, as described above, and examine the distribution of companies in the space of $(L, K)$.

We will start by considering profits. A company usually pays for the cost of its capital, $K$, in various forms, which we summarise here by an interest rate $r$. Also the company has to compensate for labour $L$ of employees. Thus the profit $\Pi$ is given by

$$
\Pi=F(L, K)-r K-L .
$$

(Here the quantity of labour $L$ is measured by a unit of money, say dollars, rather than in terms of a number.) Now, the company operates under various constraints in making decisions. This can be revealed by an attempt to maximise the profit of the above equation by choosing the amounts of its capital $K$ and labour $L$. We call this strategy profit maximisation with respect to all the factors of production. 
In fact, we can prove that profit maximisation with respect to all the factors simply does not work, if the production function is an extensive quantity as shown in the preceding section. Actually, the calculation following from the maximisation with respect to all the factors results in an interest rate $r$ which is equal to $20 \%$ or so, which is unrealistic by an order of magnitude. That is to say, companies could not in reality accept such high interest rates and gain positive profits. (See the Mathematical column on page 104 for further exposition.)

If profit maximisation with respect to all factors is non-existent, how can the present framework enable us to understand the basic strategy of companies intending to earn profits?

An obvious possibility is that, for one reason or another, a company can only change its amount of capital relatively slowly. We could then reasonably ask if it is possible to maximise profit by changing the labour $L$, only, while maintaining a fixed capital $K$. One can determine, under the validity of extensive production function, the ratio $x=K / L$, if we assume the interest rate is given by the average for the companies. Another possibility is clearly that a company may have difficulties in changing the labour input, but can easily control the amount of capital. Also, in this case, the ratio $x$ can be determined by maximisation. (The calculation for these two methods of maximisation is explained in the Mathematical column on page 104.) 


\section{$\alpha_{\beta}^{\gamma}$ Extensivity and maximisation of profit}

As has already been noted, the extensive nature of the production function can be stated in the way that the function can be written as

$$
F(L, K)=L f(x),
$$

where $x=K / L$. Putting this into the profit equation, we have

$$
\Pi=L(f(x)-r x-1) \equiv L \pi(x) .
$$

Here we use the notation $\pi(x)=f(x)-r x-1$.

Profit maximisation with respect to all the factors of production implies that the profit $\Pi$ is maximised with respect to $K$ and also with respect to $L$. The former maximisation for $K$ can be written as

$$
0=\left.\frac{\partial \Pi}{\partial K}\right|_{L}=\left.\frac{1}{L} \frac{\partial \Pi}{\partial x}\right|_{L}=\pi^{\prime}(x) .
$$

And the maximisation for $L$ can be put as

$$
0=\left.\frac{\partial \Pi}{\partial L}\right|_{K}=\left.\frac{\partial \Pi}{\partial L}\right|_{x}+\left.\left.\frac{\partial x}{\partial L}\right|_{K} \frac{\partial \Pi}{\partial x}\right|_{L}=\pi(x)-x \pi^{\prime}(x) .
$$

To maximise profit with all the factors of production, it suffices to require both of these conditions. As a consequence, it follows that $\pi(x)=0$, and equivalently $\Pi=0$, i.e. that the profit is zero at maximum, so is negative elsewhere, a state of deficit. Also, the value for $r$ is determined by two conditions, as noted in the main body of text.

If either $L$ or $K$ is fixed, and the non-fixed variable is changed to maximise the profit, only one of the two conditions is required, so we can avoid the nonsensical solution of vanishing profit. In this case, if we assume the value of $r, x$ can be determined by one of the two conditions, whose values are illustrated in Figure 3.23.

Figure 3.23 shows the consequence of our consideration in terms of the determined ratios $x$ to be compared with the actual distribution for $x$. The actual values are concentrated mostly from 1 to 2 , with the peak of the distribution approximately located at 1.5. On the other hand, the ratios calculated by maximisation with respect to either capital or labour deviated considerably from the peak. A trivial consequence of this observation is that companies fix neither capital nor labour, but change both to some degree in seeking profits, so the actual $x$ values are scattered mostly between the two extremes.

Of course, it must be admitted that this overly rough conclusion gives us little insight into any mathematically useful understanding of company production, even if we understand the properties of the production function. At this point we may wonder whether any further progress is possible in understanding the distribution of $x$ ? The answer is yes, but the key to this matter resides in ridge theory.

To understand the concept of a "ridge", think of a mountain climber. A company aiming at larger profits is like a climber attempting to reach a point 


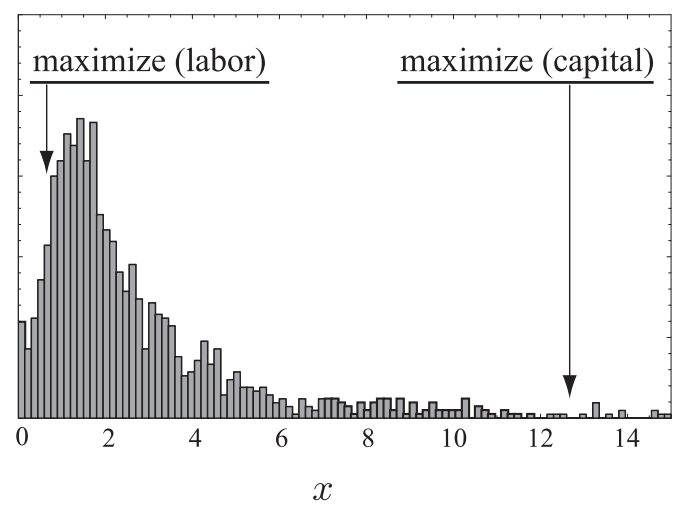

Figure 3.23: Distribution of company's $x$ and two values of $x$ at which profit is maximised under different constraints.

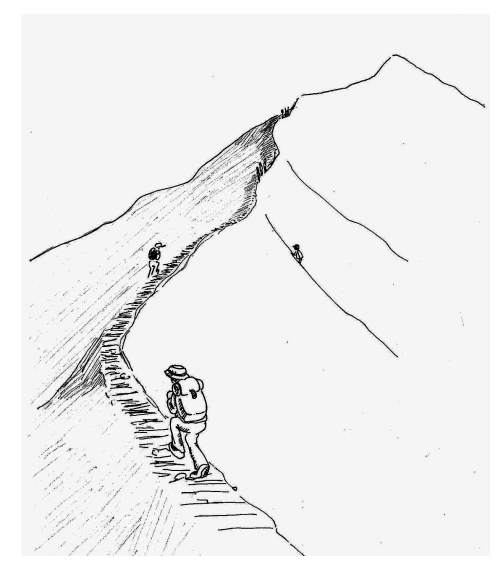

Figure 3.24: Mountain-view with a ridge. 


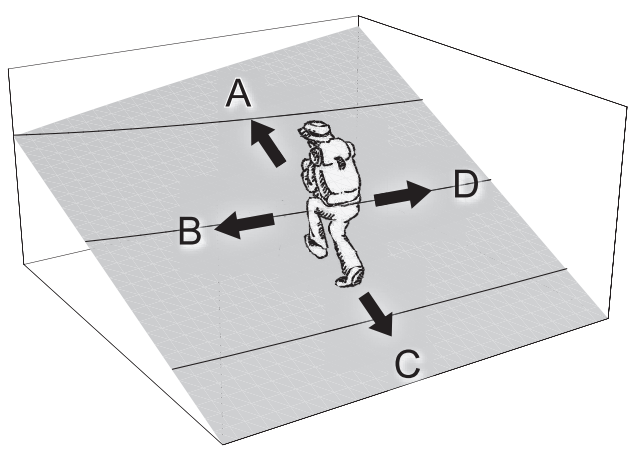

Figure 3.25: A mountain-climber and his or her directions.

further up the mountain.

Consider the terrain illustrated in Figure 3.24. This terrain includes a ridge or a long area of high land towards the top of the mountain. Suppose you are a climber standing somewhere in this terrain and want to get to a higher place as efficiently as you can. Your best bet is to take the steepest route from the place in which you are standing. Figure 3.25 draws the climber in enlargement. The direction $\mathbf{A}$ is the steepest ascent, $\mathbf{B}$ and $\mathbf{D}$ are two directions with no gradient, and $\mathbf{C}$ is the steepest descent. The directions $\mathbf{A}$, $\mathbf{C}$ are perpendicular to contours, while the directions $\mathbf{B}, \mathbf{D}$ are tangential to contours. You will walk along the direction A, perpendicularly to contours, so along the steepest-ascent line, and will eventually run into the ridge, along which you will head for the mountain's top. Some climbers may be distracted and deviate from the most efficient path, yet, after a while, all climbers will end up along the ridge.

We can expect from this analogy that the companies will be distributed along the ridge in the terrain of profit.

Finding the ridge in our case is a non-trivial task. In the terrain depicted in Figure 3.24 the ridge can be easily identified by eye, and the profit has a gentle terrain, as shown in Figure 3.26. (Note that this figure shows the surface corresponding only to the positive profit. The white and flat segment near the axis of $L$ is actually below the horizontal plane, where the profit is negative, i.e. a state of deficit.) How can we find the ridge in this terrain? The answer is to use a ridge equation, that is to say an equation which defines the ridge as a curve connecting points, each of which gives the least gradient among the steepest-ascent directions on the contour where the point is located. Details of the equation determining the ridge are given in the Mathematical column on page 109. By solving the ridge equation, one can find the ridge in 


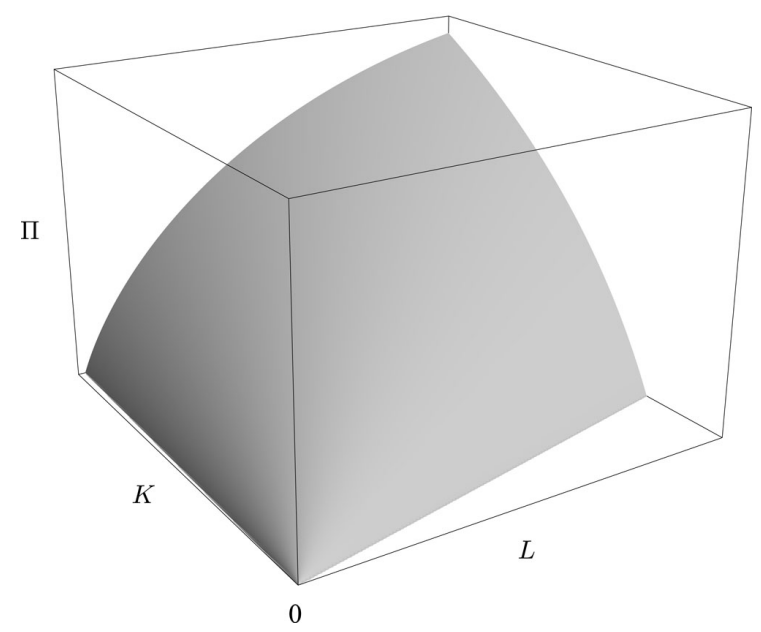

Figure 3.26: Landscape for the profit function $\Pi$.

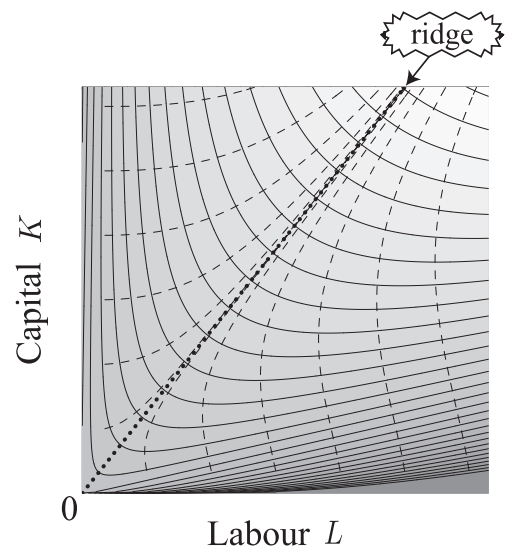

Figure 3.27: Contour lines, steepest ascent lines and a ridge for the profit landscape of Figure 3.26.

any terrain.

The actual solution of the ridge equation for Figure 3.26 is depicted in the contour plot of Figure 3.27, where higher places are drawn in a lighter colour, 


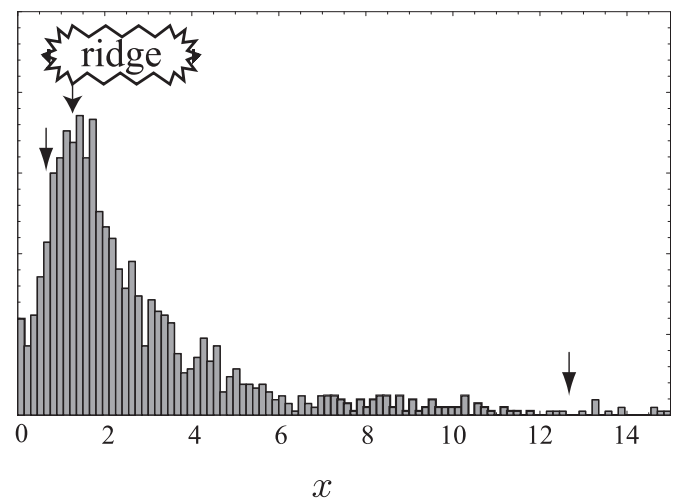

Figure 3.28: Distribution of company's $x$ (Figure 3.23) and the solution of $x$ corresponding to the ridge.

and dashed lines are "steepest-ascent lines", which an efficient climber would take at each point in the terrain. It is clear that these steepest-ascent lines are perpendicular to the contour lines, and it should also be noted that these lines converge asymptotically into the curve of ridge depicted by a dotted line. Our proposition is that the profit seeking behaviour of companies can be described by this picture.

If the production function satisfied the property of extensivity, the ridge equation determines the ratio $x$. Consequently, the curve of the ridge is actually a line, as shown in Figure 3.27. If we add this value of $x$ in our previous plot for the distribution of $x$, Figure 3.23, we have Figure 3.28. We can see that the peak in the distribution is located close to the ridge. Of course, the distribution of $x$ is spread around and centred on the ridge, and this can be understood from the fact that the ridge is the place to which the steepest-ascent lines asymptotically approaches, but do not cross into, and that companies for various reasons are presumably subject to swings and distracting bounces from various endogenous and exogenous reasons. We conclude, therefore, that one can model such fluctuations in a stochastic formulation, and make a bridging explanation to assist in understanding the distribution of companies.

In conclusion, we have seen in this section that the quantitative analysis of the production function offers considerable insight into the distribution of companies and their strategies, and leads us to a more complete sketch of the statistical universe of companies. In the next chapter we will try to add colour to this picture by drawing on the theory of business network among companies.

\section{$\alpha_{\beta}^{\gamma}$ Ridge equation}


To write down a ridge equation in a general form, it would be more comprehensible to consider $n$ variables, $\phi_{i}(i=1,2, \ldots, n)$, for the function of profit $\Pi$. This is so although what we actually need to consider is the case, $\left(\phi_{1}, \phi_{2}\right)=(L, K)$, in two-dimension $n=2$.

In this notation, the ridge equation can be given by

$$
\sum_{j=1}^{n} \frac{\partial^{2} \Pi}{\partial \phi_{i} \phi_{j}} \frac{\partial \Pi}{\partial \phi_{j}}=\lambda \frac{\partial \Pi}{\partial \phi_{i}} .
$$

Mathematically, this is an eigenvalue equation for the second-derivative of $\Pi$, and the eigenvalue $\lambda$ is a parameter on the curve of the ridge solution. That is, for a given $\lambda$ (in an appropriate range), the solution of the ridge equation gives a set of coordinates $\left(\phi_{1}, \phi_{2}, \ldots, \phi_{n}\right)$, or a point in the space, the location of which varies by changing the value of $\lambda$ resulting in the curve of a ridge.

The above equation can also be interpreted as an extremisation of the length of the gradient vector $(\partial \Pi / \partial \phi)$ under the constraint that $\Pi=$ constant, which is easily proved by the argument of the "Lagrange multiplier". From the solutions of the ridge equation we need to choose the minimum of the extreme values in this argument, which correspond to ridge.

Note that ridge and steepest-ascent line are different concepts. A steepest-ascent line is along the gradient or the direction perpendicular to the contours. In other words, since it is necessarily obtained by requiring that it be parallel to the gradient vectors, a steepest-ascent line is defined by the differential equation:

$$
\frac{d \phi_{i}(s)}{d s}=\frac{\partial \Pi}{\partial \phi_{i}} \quad(i=1,2, \ldots, n) .
$$

Here $s$ is a parameter on the line of steepest-ascent. This is a differential equation with respect to the spatial coordinates, $\left(\phi_{1}, \phi_{2}, \ldots, \phi_{n}\right)$, with an arbitrary choice of initial values for those coordinates. Thus we have an infinite number of steepestascent lines for choices of initial-values. The dotted line in Figure 3.27 is drawn by solving this equation for different choices of initial values. See Aoyama and Kikuchi (1992) and Aoyama et al. (1999). 


\section{Chapter 4}

\section{Complex Business Networks}

Perhaps everyone has asked themselves if there is there anything in the universe that is perfectly isolated, separate and complete within itself. Some will answer that the universe itself is the only thing, because the universe alone is unique. However, the physics of elementary particles and cosmology suggests the possibility that many universes similar to our universe exist and interact with each other through quantum gravity.

The appearance of a discussion of the universe or universes in a book on economic theory may seem abrupt and even odd, but if we recast the point in terms that are more familiar it won't seem so peculiar. When strangers meet, at a party or by chance on a train, it is not uncommon for them to find that they have shared acquaintances. "It's a small-world!", they will exclaim, in genuine surprise. In fact this happens all the time, so it is not so much the frequency that stimulates our wonder, but the reasons which underlie it, and these are the subject of long-standing and unsolved questions.

In an attempt to shed light on this matter, sociologists have conducted many kinds of experiments, and needless to say this attempt requires an understanding of human networks. However, it is as important to extend our outlook and to consider the more abstract and far-reaching question: "What is a network?" In other words, when we cannot solve a problem in a particular and special case, we can sometimes gain insight by generalising. Networks are just such a case.

The history of the train of thought grounded on this viewpoint is not long, indeed it started only very recently under the title of network science, and is making remarkable progress (Barabási, 2003; Buchanan, 2003; Watts, 1999, 2003; Caldarelli, 2007). In the next section, we provide a brief introduction to this new field. 


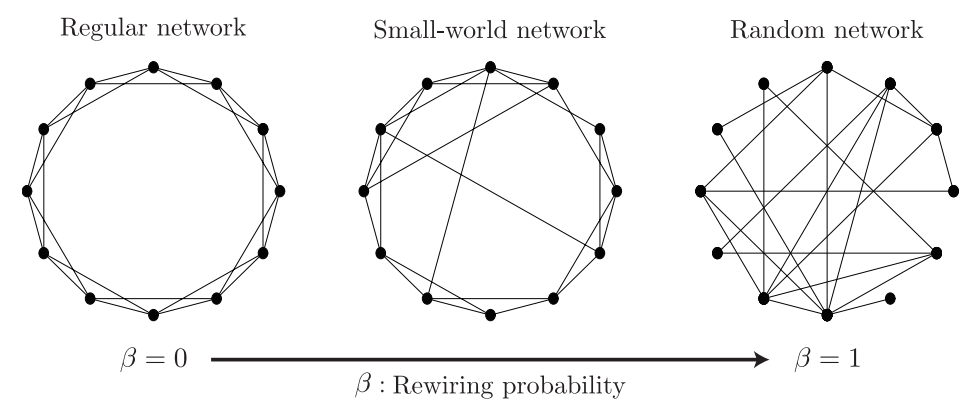

Figure 4.1: Watts-Strogatz $\beta$ model

\subsection{Introduction to Network Science}

The origin of network science is graph theory in mathematics, social network analysis in sociology, statistical mechanics in physics, and computer science. Historically, graph theory came first, with its origin being Euler's solution of the Köningsberg bridge problem in 1736. Subsequently, the theory was developed by various figures of enormous distinction, Pólya, Erdős, and others.

A graph is constructed from dots and lines. In network science, a dot is called a node or a vertex, and a line is called an edge or a link. In this book, we use the terms "node" and "link", except in certain special cases. In addition, we use the terms "graph" and "network" interchangeably, as having the same meaning.

The field of graph theory has concentrated its investigation on regular networks, trees, and random networks. The regular network has nodes with an equal number of links, and these nodes are connected in a regular fashion. For example, the left panel of Figure 4.1 displays a regular network. In this figure, each node has four links, and these links connect each node with its two nearest neighbours both right and left. Hence, in a regular network, every node is equal and there is no special node. A tree is a graph which does not contain loops, and its topology is same as trees and rivers in the real world. A random network, on the other hand, is constructed by connecting nodes completely at random. For example, the right panel of Figure 4.1 displays a random network. ${ }^{1}$ Graph theory proved various theorems for these networks, but the instances it investigated were quite different from real world networks, for example social networks.

Social network analysis in sociology has experienced two revolutionary developments, firstly in the 1930s, and secondly in the 1960s through to the 1970s (Freeman, 2004). Between these two revolutionary developments, Stan-

\footnotetext{
${ }^{1}$ Strictly speaking, that shown here is a Watts-Strogatz random network, one of several classes of random networks.
} 
ley Milgram famously experimented three times to measure the size of the real social world (Milgram, 1967), and consequently his name is strongly associated with the terms "small-world", and "six degrees of separation" However, there are many sociologists who do not accept Milgram's interpretation of his experiments, and some sociologists have asked whether the real world is actually small or not?. In distinction from graph theory, social network analysis has investigated networks in the real world, but the size of networks considered is comparatively small, not exceeding the order of $10^{3}$.

However, recently, several sets of network data have become available as readily accessed computerised databases, for example, the gigantic network of the internet itself, a development which switched the target of study from small to large networks.

It was in this context that Watts and Strogatz published their seminal paper in Nature, and so shaped network science as we currently know it (Watts and Strogatz, 1998). The paper introduced the concepts of averaged path length and the clustering coefficient to characterise networks.

The averaged path length is the average number of links which connect a pair of randomly selected nodes with the shortest path. Let us denote the number of links which is necessary to connect node $i$ and $j$ with the shortest path as $l_{i j}$. By using $l_{i j}$, for node $i$, we can calculate the mean value of the shortest path, $L_{i}$. Thus, the averaged path length, $L$, of the subjecting

\section{Milgram's positivism}

Milgram is often thought to be the 20th century's characteristic psychologist, less for "six degrees separation" than the notorious "obedience to authority" experiment, in which naive subjects were given apparent control over the application of, in fact false, electronic shocks to a third person. The purpose was to investigate our capacity to commit inhumane acts when legitimated by a person in authority.

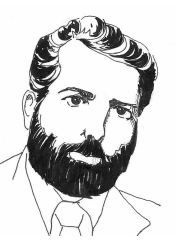

The result of the experiment suggested that almost all of us, independent of socioeconomic status and sex, are capable of great cruelty if authorised, a result that was contradictory to the preliminary expectations of many psychologists and caused a sensation in 1963 when the paper was published.

As is clear from this experiment, Milgram's approach to science was to proceed empirically without relying unduly on plausible but weakly grounded discussion and prejudice. In our investigation of the relation of economy and society, Milgram's attitude will be one of our guiding principles. 
network is given by the averaged value of $L_{i}$, i.e.,

$$
L_{i}:=\frac{1}{N-1} \sum_{j \neq i} l_{i j}, \quad L:=\frac{1}{N} \sum_{i=1}^{N} L_{i} .
$$

where $N$ is the total number of nodes.

If three nodes are connected with each other, i.e., if two of my friends are also friends with each other, this configuration forms a triangle, which we will call a cluster. The clustering coefficient quantifies the cliqueyness of nodes, and is given by the probability of finding triangles in the network. If the node $i$ has $k_{i}$ links, the number of possible triangles is given by the number of the combinations in which two nodes can be selected from $k_{i}$ nodes, i.e., $C\left(k_{i}, 2\right)=k_{i}\left(k_{i}-1\right) / 2$. However, in a real network, only a fraction of these possible combinations is accomplished. The clustering coefficient of node $i$, i.e. $C_{i}$, is defined by dividing the number of really existing clusters, $E_{i}$, by the number of possible clusters. Thus, the clustering coefficient of the network, $C$, is given by

$$
C_{i}:=\frac{2 E_{i}}{k_{i}\left(k_{i}-1\right)}, \quad C:=\frac{1}{N} \sum_{i=1}^{N} C_{i},
$$

i.e., it is the averaged value of $C_{i}$. (See the column on page 114). By convention, $C_{i}=0$ for the trivial cases $k_{i}=0$ or 1 .

Watts and Strogatz calculated the averaged path length $L$ and the clustering coefficient $C$ for the collaboration graph of actors in feature films, the

\section{$\alpha_{\beta}^{\gamma}$ Global clustering coefficient}

We can define another type of clustering coefficient called a global clustering coefficient. This differs from the clustering coefficient defined in the main text. The global clustering coefficient is defined by

$$
C_{\text {global }}=\frac{3 \times(\text { number of triangles in the network })}{\text { number of connected triplets of nodes }} .
$$

If "Mr. A" has two friends ,"Mr. B" and "Mr. C", we refer to this configuration as a connected triplet of nodes, and denote it as "B-A-C" (or "C-A-B"). Additionally, if "Mr. B" and "Mr. C" are also friends with each other, we can construct the triangle $\triangle \mathrm{ABC}$. This triangle contains three connected triplets of nodes, i.e., (A-B$\mathrm{C}, \mathrm{B}-\mathrm{C}-\mathrm{A}, \mathrm{C}-\mathrm{A}-\mathrm{B})$. The factor of 3 in the above equation makes $C_{\text {global }}=1$ in this situation.

Incidentally, $C_{i}$ is explained in the main text as being equivalent to the probability that the friends of node $i$ are also friends with each other, and where $C$ is the averaged value of this probability. On the other hand, $C_{\text {global }}$ gives the probability that a randomly selected triplet forms a triangle. $C$ and $C_{\text {global }}$ generally have different values (Newman, 2003c). One must be careful which definitions of clustering coefficient are applied in any particular case. 
electrical power grid of the western United States, and the neural network of the nematode worm, C. elegans (Watts and Strogatz, 1998). Their results suggested that these networks have both the short averaged path length that is characteristic of random networks (see the column on page 115) and a large clustering coefficient that is characteristic of regular networks (see the column on page 116). They referred to networks with this combination of characteristics as small-world networks (see the column on page 116).

The right panel of Figure 4.1 is an example of a random network, and shows that only a few links connect a pair of randomly selected nodes, and also that there is a low probability finding triangles. Thus, a random network has a short averaged path length and a small clustering coefficient. On the other hand, the left panel of Figure 4.1 is an example of a regular network. Contrary to a random network, this figure shows that two randomly selected two nodes are connected by many links, and that the probability of finding triangles is high. Hence, a regular network has a long averaged path length and a large clustering coefficient.

Watts and Strogatz also proposed a so-called $\boldsymbol{\beta}$ model, which aims to answer the small-world problem, as discussed in the sociology (Watts and Strogatz, 1998; Watts, 1999). In their model the links of the regular network shown in the left panel of Figure 4.1 are rewired with the probability $\beta$. Thus, the case of $\beta=0$ is a regular network. By changing the rewiring probability

\section{$\alpha_{\beta}^{\gamma}$ Poisson random network}

The network proposed by Erdős and Rényi is another example of a random network (Erdős, P. and Rényi, A., 1960), this time one where the probability with which two nodes are connected is constant $\theta$ and independent of the existence of other links. In this case, we can easily see that by definition the probability $p(k)$ of finding a node with the degree $k$ is given by

$$
p(k)=C(N-1, k) \theta^{k}(1-\theta)^{N-1-k},
$$

where $N$ is the total number of nodes.

If we take the limit $N \rightarrow \infty$ under the constant averaged degree $\langle k\rangle=N \theta=: z$, we have $p(k) \simeq z^{k} e^{-z} / k$ !, where $k$ ! is a factorial of $k$. Thus, the degree distribution is given by $p(k) \simeq z^{k} e^{-z} / k$ !, which is a Poisson distribution, and the network is a Poisson random network.

In a Poisson random network the averaged path length $L_{\text {rand }}$ and clustering coefficient $C_{\text {rand }}$ are given by

$$
L_{\mathrm{rand}} \simeq \frac{\log N}{\log z}, \quad C_{\mathrm{rand}} \simeq \frac{z}{N},
$$

respectively (see Bollobás, B. (1985); Newman (2003b) for example). The defining characteristic of a random network is that the averaged path length is proportional to the logarithm of the network size, namely $\log N$. On the other hand, the clustering coefficient is proportional to the size of network, in other words $N$. 
$\beta$ we can reproduce a small-world network. That is to say, a network with $\beta=1$ would belong to the class of random networks. ${ }^{2}$ The $\beta$ model suggested that the existence of links corresponding to short cuts is what turns the real world into a small-world.

A little earlier we said that the paper written by Watts and Strogatz was the first paper on network science, but it might be more accurate to say that the paper turned the key of the door to network science without quite opening it. That honour goes to A.-L. Barabási. whose research group at Notre-Dame University wrote a series of papers in 1999 analysing the structure of the World Wide Web (Albert et al., 1999; Barabási and Albert, 1999). They found that the degree distribution of that network follows a power-law distribution, and they refer to such networks scale-free. Here, the degree

\footnotetext{
${ }^{2}$ The difference between the random network made by rewiring with $\beta=1$ and that proposed by Erdős and Rényi is degree distribution.
}

\section{$\alpha_{\beta}^{\gamma} \quad$ Regular network}

As shown in the left panel of Figure 4.1, a regular network is constructed by regularly arranging nodes with an equal number of degrees, $k_{0}$. In a one dimensional and regularly linked network the averaged path length $L_{\mathrm{reg}}$ and the clustering coefficient $C_{\text {reg }}$ are given by

$$
\begin{aligned}
L_{\mathrm{reg}} & =\frac{N\left(N+k_{0}-2\right)}{2 k_{0}(N-1)} \simeq \frac{N}{2 k_{0}}, \\
C_{\mathrm{reg}} & =\frac{3\left(k_{0}-2\right)}{4\left(k_{0}-1\right)},
\end{aligned}
$$

respectively. The defining characteristic of a regular network is that the averaged path length is proportional to the size of network, namely $N$. On the other hand, the clustering coefficient is completely independent of the size of the network, and is given by $C_{\text {reg }} \simeq 3 / 4=0.75$, when $k_{0}$ is comparatively large. In addition the degree distribution for a regular network is given by Dirac's $\delta$ function, i.e., $p(k)=\delta\left(k-k_{0}\right)$.

\section{$\alpha_{\beta}^{\gamma}$ Small world network}

Watts and Strogatz calculated the averaged path length $L_{\mathrm{sw}}$ and $C_{\mathrm{sw}}$ for some real world networks, and found that many have short average path lengths and large clustering coefficients. That is to say, these quantities satisfy

$$
\begin{aligned}
& L_{\mathrm{sw}} \simeq L_{\mathrm{rand}} \ll L_{\mathrm{reg}}, \\
& C_{\mathrm{sw}} \simeq C_{\mathrm{reg}} \gg C_{\mathrm{rand}},
\end{aligned}
$$

respectively. At present, networks with these characteristics are referred to as the small-world networks of Watts-Strogatz, or, more simply, small-world networks. In the $\beta$ model of Watts-Strogatz, the degree distribution changes from Dirac's $\delta$ function to the Poisson distribution, by changing the rewiring probability $\beta$. 
is defined by the total number of links held by a node (See the column on page 117). In Section 4.5.2, we will show that some economic networks are also scale-free networks.

Scale-free phenomena were first observed in the year 1897 when Pareto found the power-law distribution in the wealth distribution of the rich (see Chapter 2). Scale-free phenomena were also observed in the study of phase transition and critical phenomena, Mandelbrot's fractals, and the study of complex systems, all of which have a long history. Many physicists have been fascinated by these phenomena, and had already studied them for some decades before moving smoothly across to the examination of scale-free networks.

Subsequently, many concepts and analytical methods were imported into network science from the fields such as biology, life science, and sociology. Simultaneously, the number of scientists studying network science increased rapidly, and at present the study of networks is interdisciplinary and integrated. It is also practical.

One example of the application of network science to the real world is the concept of hub and authority, which was introduced by J. Kleinberg while studying the Internet (Kleinberg, 1999). Another would be the concept of PageRank proposed by Brin and Page (Brin and Page, 1998), ${ }^{3}$ a concept

${ }^{3}$ Curiously, "Page" in the word "PageRank" refers to the name of its discoverer, not a "page" of a book or a web page.

\section{$\alpha_{\beta} \gamma$ Degree distribution and others}

Degree is the total number of links held by each node. A node with large degree is called a hub. The degree distribution $p(k)$ is the probability that a randomly selected node in the network has degree $k$.

Sometimes each hub is connected to every other and sometimes not. The degree correlation quantifies the correlation between the degrees of two nodes connected by a link. For example, we can study the joint distribution for a pair of degrees, and calculate their correlation coefficients (See the column on page 121).

If we use qualitative or quantitative characteristics of nodes (for example, race or age) instead of degree, we can examine what kinds of features effect the existence of links connecting a pair of nodes. That is to say, if we cut links and connect nodes randomly, we lose information embedded in the characteristics of links; therefore, the correlation between nodal characteristics is useful in determining network character. When the probability of constructing a link between nodes with similar characteristics is higher than that between nodes with different characteristics, we refer to the former case as assortativity (see Newman (2003c) and references therein).

There are other indices characterising networks, for example, the correlation between degree and clustering coefficients, hierarchical structure (Barabási and Oltvai, 2004), many kinds of centralities, network motifs (Milo et al., 2002; Shen-Orr et al., 2002), network spectrum, and others. 
responsible for the beginnings of the Google search engine.

The development of network science also has connections with changing social conditions. The terrible September 11 attacks have had profound consequences, and there is now widespread fear of terrorist networks spanning the world, a previously uncontemplated diversified network. Similarly, epidemics such as SARS, bird flu, and swine flu, the expanding virtual internet society, and arguably dwindling relations between individuals have drawn attention to the importance of understanding the world as complex networks.

At present the concerns of network science are the study of large networks, new network indices, ${ }^{4}$ fast algorithms for the calculation of network indices, dynamics in the network, mechanisms for the generation of networks, and the visualisation of networks, amongst others. In addition, how to apply the results of network science to the real world is also an important problem. In the international conference held in Aveiro, Portugal, in 2004, one of organizers, S. Dorogovtsev, referred to the need to move "beyond Google". It will take a long time to apply network science to the real world on a broad scale, but the possibility of that outcome is gradually increasing.

\section{$4.21,2,3, \ldots, 6$ degrees of separation}

We earlier referred to Milgram's "six degrees of separation", and explained that the $\beta$ model gives an answer to the small-world problem. Here, we explain the method for calculating the averaged number of nodes within a given number of degrees of separation for both real world and theoretical networks. This not only gives another intellectual perspective on small-world phenomena, but also includes an important suggestion towards understanding the connection of nodes in a complex network.

We will start with "one degree" of separation, instead of jumping in at "six". In short, a node within "one degree of separation" is a node directly connecting to an arbitrary node with one link. Therefore, the average number of nodes in one degree of separation is equal to the "averaged degree", that is the averaged number of nodes within one degree of separation. In a complete graph, in which every node is connected to every other node, the averaged number of nodes equals the total number of nodes minus one. On the other hand, in complex networks the averaged number of nodes is of order one. Many social or economic networks are huge, and the range of the total number of nodes is from $10^{5}$ to $10^{8}$ and there is practically no possibility that all nodes can be connected to each other.

Let us now turn to "two degrees of separation". In the case of all nodes with an equal degree, $k_{0}$, an arbitrary node is connected by $k_{0}$ nodes, each of which is connected with $k_{0}-1$ nodes, except the original node. Thus the number of nodes within "two degrees of separation" is $k_{0}\left(k_{0}-1\right)$. If we believe that this estimation is correct in the case of a large number of nodes, we might

\footnotetext{
${ }^{4} \mathrm{An}$ index characterising network is called a network index. The previously introduced topics of averaged path length, clustering coefficient, and degree are also network indices.
} 


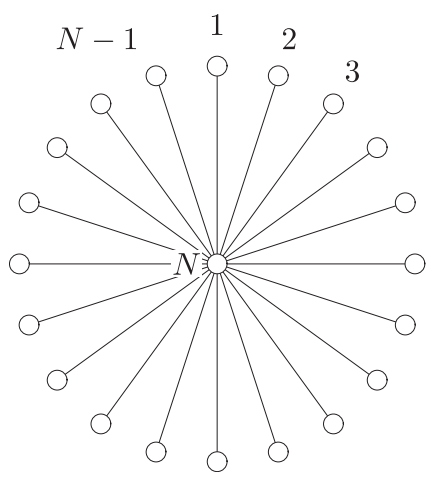

Figure 4.2: A complete graph in which every node is connected to every other within "two degrees of separation"

say that the averaged number of nodes within "two degrees of separation" is approximately given by

$$
\langle k\rangle(\langle k\rangle-1),
$$

by replacing $k_{0}$ to the averaged value $\langle k\rangle$. However this estimation is completely wrong as shown in what follows.

A simple example illustrating this point is shown in Figure 4.2. In this network, the node in the centre of network is connected to every other node. Thus, the degree of the centre node is $N-1$, and that of the other $N-1$ nodes is 1 . Hence, the averaged degree is given by

$$
\frac{1 \times(N-1)+(N-1) \times 1}{N}=\frac{2(N-1)}{N} \simeq 2,
$$

being approximately equal to 2 for large $N$. If we substitute this value for our naive estimate for the averaged number of nodes within "two degrees of separation", we get

$$
\langle k\rangle(\langle k\rangle-1)=2(2-1)=2 .
$$

However, as is apparent from the figure, all $N-1$ dead-end nodes are connected with all $N-2$ dead-end nodes through the centre node within "two degrees of separation". (The centre node does not have nodes within two degrees of separation.) Thus, if we calculate the averaged number of nodes within two degrees of separation, the result is approximately equal to $N$ when $N$ is sufficiently large, which is completely different from the naive estimate obtained above.

Actually, for general networks that contain nodes with different degree values, we can calculate the number of nodes within two degrees of separation more precisely. By using the square average of degree of node $\left\langle k^{2}\right\rangle$, the result is given by

$$
\left\langle k^{2}\right\rangle-\langle k\rangle \text {. }
$$


Of course, if the degree of nodes is uniformly $k_{0}$, the result is given by $\left\langle k^{2}\right\rangle=$ $\langle k\rangle^{2}=k_{0}^{2}$ which agrees with the naive estimate. However, in general, $\left\langle k^{2}\right\rangle$ is larger than $\langle k\rangle^{2}$, and the difference between them is given by a variance. Thus, the simple estimation given previously is an underestimate. (See Newman (2003a) for the exposition of this fact.)

In fact, in the example of Figure 4.2, because of existence of the centre node, the magnitude of the square average of degree $\left\langle k^{2}\right\rangle$ is approximately $N$. As a result, if we calculate the average number of nodes within two degrees of separation by using the solution stated above, we obtain the correct result which is approximately equal to $N$.

From this example, we can see why the average number of nodes within two degrees of separation becomes large, as stated below;

- Nodes can be connected to many nodes within two degrees of separation through directly connected nodes with a large degree (a hub).

- Nodes with a large degree (hubs) can take such an intermediating role in many cases because they are connected to many other nodes.

To put it simply, the number of your friends is much larger than that which you naively expect, because you have a non-negligible probability of finding a hub amongst your friends. The probability is non-negligible due to the fact that she or he has many friends, and in spite of the fact that the number of such hubs is quite small. The number of your friends' friends is, therefore, much larger than that might be expected.

Actually, the real-world networks discussed so far have fat tails and large variance, and the number of nodes within two degrees of separation can be much larger than the above estimate. In fact, the tail of the degree distribution for scale-free networks follows a power-law distribution:

$$
P_{>}(k) \propto k^{-\nu},
$$

and therefore, if the power-law exponent $\nu$ is less than $2,\left\langle k^{2}\right\rangle$ diverges and becomes infinite. However, since a divergence of $\left\langle k^{2}\right\rangle$ occurs in the case of ideal infinite networks, it is clear that, therefore, such a divergence does not happen in real, finite, networks. Precise estimation clarifies that the square average of degree $\left\langle k^{2}\right\rangle$ becomes the magnitude of the total number of nodes, when the power-law exponent $\nu$ is equal to 1 . Thus, in scale-free networks, every node connects with another within two degrees of separation, when exponent $\nu$ equals to 1 . However, this is not accomplished in the real world, because actual scale-free networks do not have such a small $\nu$.

Now, if we move to the discussion of three degrees of separation, new evidence is added to the discussion of two degrees of separation and it becomes more interesting, though at this point we will simply display the result. The most effective term for the number of nodes within three degrees of separation 
is given $b y^{5}$

$$
r\left\langle k^{3}\right\rangle
$$

$r$ can be referred to as the correlation coefficient of degrees, and characterises the correlation between degrees which are for two nodes attached to each end of a link (See Figure 4.3). If degrees have no correlation, $r=0$. In scale-free networks, when $\nu=1.5$, then the average of the cube of degree $\left\langle k^{3}\right\rangle$ approaches to the total number of nodes, i.e., $N$. Thus, as long as the degree correlation is not equal to zero, every node is connected with every other within three degrees of separation when $\nu$ is below 1.5. Incidentally, if there is no correlation between degrees and $r=0$, every node is connected with another within three degrees of separation when $\nu$ is below $4 / 3$. From this result, we can see the importance of the correlation between nodes.

Now, if we expand the calculation to the case of six degrees of separation we get a very complicated and long equation, needing many pages to display. ${ }^{6}$ If we pick up the effective part of the equation, which contains the degree correlation coefficient $r_{4}$ of nodes connecting within four degrees of separation (See Figure 4.4), we get

$$
r_{4}\left\langle k^{17}\right\rangle^{1 / 8}
$$

This quantity is of the same order as the total number of nodes when $\mu$ is less than or equal to 3 ; therefore the subject of this section, "six degrees of

\footnotetext{
${ }^{5}$ We here show the equation which is applicable when clustering coefficient is sufficiently small.

${ }^{6}$ It is impossible to calculate it by hand, so we used the Mathematica software for algebraic calculation and a fast $\mathrm{PC}$ with $8 \mathrm{~GB}$ memory; even so, it took over ten minutes to get the result.
}

\section{$\alpha_{\beta} \gamma^{\prime}$ Correlation coefficient}

The correlation coefficient is defined as follows. We suppose that a random variable $x$ has a mean $\langle x\rangle$ and standard deviation,

$$
\sigma_{x}=\sqrt{\left\langle(x-\langle x\rangle)^{2}\right\rangle}=\sqrt{\left\langle x^{2}\right\rangle-\langle x\rangle^{2}} .
$$

If we define a new variable $\tilde{x}$ as $\tilde{x}:=(x-\langle x\rangle) / \sigma_{x}$, this new variable $\tilde{x}$ has the mean equal to 0 and the standard deviation equal to 1 . For another variable $y$, we define $\tilde{y}$ in the same way. The average of the product of these two variables:

$$
r_{x y}:=\langle\tilde{x} \tilde{y}\rangle=\frac{\langle x y\rangle-\langle x\rangle\langle y\rangle}{\sigma_{x} \sigma_{y}}
$$

is called the correlation coefficient of $x$ and $y$. In addition, the numerator of the last term of the right hand side of above equation, i.e., the product of correlation coefficient and standard deviations of each variable: $\sigma_{x y}:=\sigma_{x} \sigma_{y} r_{x y}$ is called the covariance. It is easily verified that the correlation coefficient $r_{x y}$ necessarily satisfies $-1 \leq r_{x y} \leq 1$. 


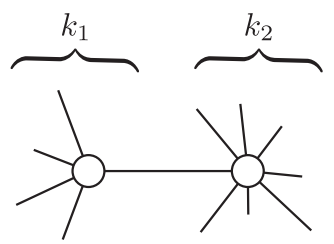

Figure 4.3: Correlation $r$ between degrees of nodes at distance 1

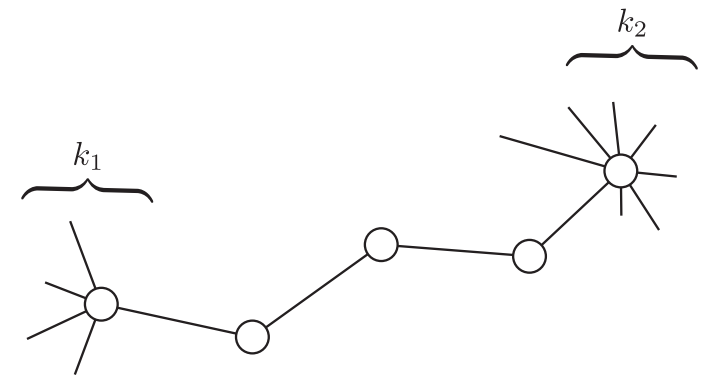

Figure 4.4: Correlation $r_{4}$ between degrees of nodes at distance 4

separation", is realised if $\mu$ is not over 3 . However, in a real network, it sometimes happens that the degree correlation becomes zero if the separation of nodes is four. Thus, we simplify the problem by assuming that only the degree correlation coefficient mediated by one link, $r$, which appeared in the case of three degrees of separation, is not equal to zero, and the degree correlation coefficient mediated by more than two links is equal to zero. In that case we find the term

$$
r^{2} \frac{\left\langle k^{3}\right\rangle^{2}}{\left\langle k^{2}\right\rangle}
$$

is effective to six degrees of separation. Such a result means that this term becomes approximately $N$ when $\nu$ is less than or equal 2.4, and all nodes are connected within six degrees of separation.

In the calculation explained above we ignored the clustering coefficient in cases with three degrees of separation or more. However, this is not likely to happen if the exponent of $N$ is modified by the effect of a clustering coefficient. Additionally, many degree distributions in real world networks have a fat tail with an exponent in the range from 1 to 3 . Thus, for this reason, it is possible to consider that all nodes are connected within the extent of six degrees of separation in the real world, if the network is scale-free.

The calculation of $d$ degrees of separation has an interesting application to the estimation of chain of bankruptcy in a supplier-customer network. As 
we shall explain in Section 6.2, companies connected by supplier-customer relationships to a bankrupted company can also fail. In the range of one degree separation from a bankrupted company, if there are no other failed nodes, the bankruptcy is isolated. However, if there is another bankruptcy in the range, we can proceed to consider two degrees of separation from the starting bankruptcy. If there is no other failure, then there is a chain of bankruptcies with size two, occurring simply by chance. Proceeding in this way, one can consider chains of bankruptcy with larger size in the supplier-customer network, and compare such an estimation for the frequency of "avalanche" size with what actually happened in a real data. See Fujiwara and Aoyama (2008) for details.

\section{Kevin Bacon game}

Do you know the "Kevin Bacon game" which originated from the six degrees of separation theory. Players search for the number of links constituting the shortest path connecting an arbitrarily selected movie star and Kevin Bacon, another actor, by tracing a path through the movie collaboration network. This is called the "Bacon number". At the end of 2008, approximately one million actors were connected to Kevin Bacon, and the average value of the Bacon number was approximately 2.95. Interestingly, if we calculate the same number for other actors, the actor who has the smallest number, i.e., the centre of the network, is not Kevin Bacon but Dennis Hopper, whose "number" is equal to 2.74. There are several other "numbers" of this kind, for example the "Erdős number" which is defined by the number of links in the collaboration network for the mathematician Erdős (introduced in pp. 112).

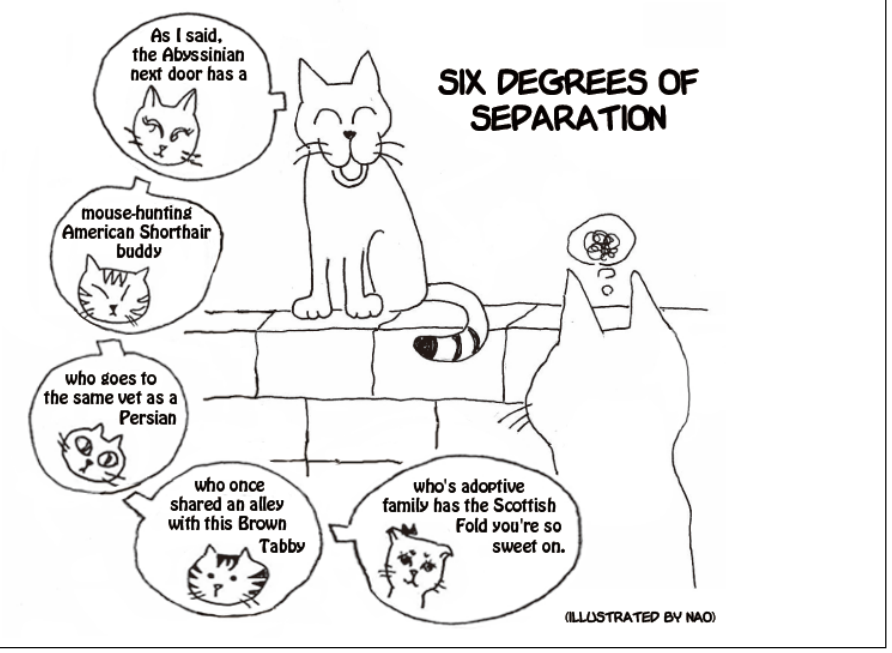




\subsection{Networks in Economy}

When discussing networks in economy it is important at the outset to define what we mean by a node in this case. If we decide that we want this to correspond to the minimum unit which is accepted as an economic entity we might propose the individual person as a candidate. In a future where electric money and IC tags are widespread, and the consumption pattern of an individual and the flows of money and goods are traceable, we will be able to study the network constructed from individuals. However, at present this is impossible.

Since, for practical reasons, we need to find another candidate definition of "node" it is obvious that we must reconcile ourselves to some degree of coarse graining. For example, we might suggest that a node could be defined as a group consisting of several persons, that is to say a company, a local government, a country, and so on. For our purposes in this book we have elected to consider the company as a node.

This decision raises the question as to what kind of relationship between companies we should consider as constituting a link. In a company limited by shares, which is the predominant conformation, ownership and management are separated. The shareholders own the company, and may hold shares in several companies. In listed companies it is frequently found that shareholders are themselves also listed companies. Thus, we can identify for consideration the shareholding network, a network in which the nodes are companies and the links are shareholding relationships.

Members of the board of directors are selected at the meeting of shareholders, and the board of directors makes decisions for the management of the company. Sometimes, some members of one board are also members of another board, and so are interlocking directors connecting two or more companies. Thus we can identify the interlocking directors' network as another company network.

Under such ownership and management, companies perform many kinds of activity, the most important of which is production. Many companies buy materials and services to make products, and sell these products with value added, so we can on the basis of these relations identify a transaction network.

The importance of innovation is a much discussed topic, but measuring it is a very difficult problem. Some traction can be obtained by considering patents as if they were innovations, and this is the approach we adopt here. Since companies sometimes apply jointly for a patent, presumably because of previous joint research, we can consider the network of joint applications for patents as evidence of an innovation network. In what follows we will consider the shareholding network, the interlocking directors' network, the transaction network, and innovation network, in sequence. 


\subsubsection{The shareholding Network}

Major shareholder data is useful for studying the shareholding connection between companies, ${ }^{7}$ and the data we have used provides the list of top thirty major shareholders for each listed company.

Stock holdings data is also useful for studying the shareholding network. In Japan, we can buy an annual security report at major book stores, or read it on the home page of Electronic Disclosure for Investors' NETwork (EDINET). An annual security report contains an investment portfolio item in which the details of a company's investment status is reported. In many cases the top ten to twenty major investment companies are reported, but the length of the disclosed list differs from company to company. The list of investment portfolios includes investment trust funds, but in this book, we ignore such funds in our examination of the shareholding network, which is restricted to major shareholder data and stock holding data in 2004.

Figure 4.5 is a conceptual picture of the connection of companies. In this figure, each white circle corresponds to a company. We draw the shareholding network by tracing an arrow from shareholder to company. Thus, major shareholder data corresponds to the left side part of Figure 4.5. In this part, each white circle existing in the left side corresponds to a shareholder, and each link describes a shareholding relationship. In other words, major shareholder data is data for links, which come into to each listed company. In this way, the link that comes into a node is called an incoming link, and the number of incoming links is called the in-degree or the incoming degree. As stated before, in the case of major shareholder data, the incoming degree is up to 30 .

Meanwhile, stock holdings data corresponds to the right side part of Figure 4.5. Stock holding data refers to the links that go out from each listed company. That is to say, a link that goes out from a node is called an outgoing link, and the number of outgoing links is called the out-degree or the outgoing degree. In the case of stock holding data, the outgoing degree differs from company to company.

By using these two kinds of data, i.e., major shareholding data and stock holding data, we get the part shown in Figure 4.5 for each listed company. The shareholding network is constructed by connecting these elements to each other.

For the purposes of the present analysis we will focus on companies concerned with automobile manufacture. According to the classification of industry by Nikkei NEEDS, industries concerned with automobile manufacture are separated into automobile (automobile), automobile (auto body and others), automobile (auto parts), and electric machinery (automobile-related), the terms in the brackets corresponding to the minor classification of automobile and electric machinery industries. We will concentrate on the automobile (automobile) industry, which for simplicity's sake we will refer to as the auto-

\footnotetext{
${ }^{7}$ Major shareholder data, for example, is commercially available from Toyo Keizai Inc. and Nikkei Data Service Inc.
} 


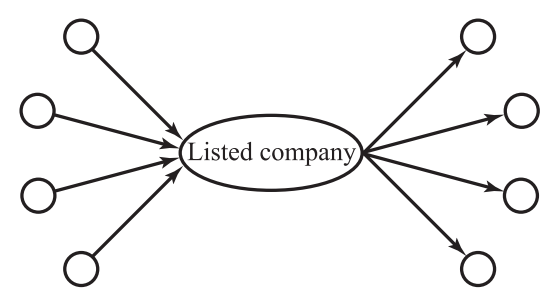

Figure 4.5: Incoming and outgoing links of a listed company in a shareholding or a transaction network

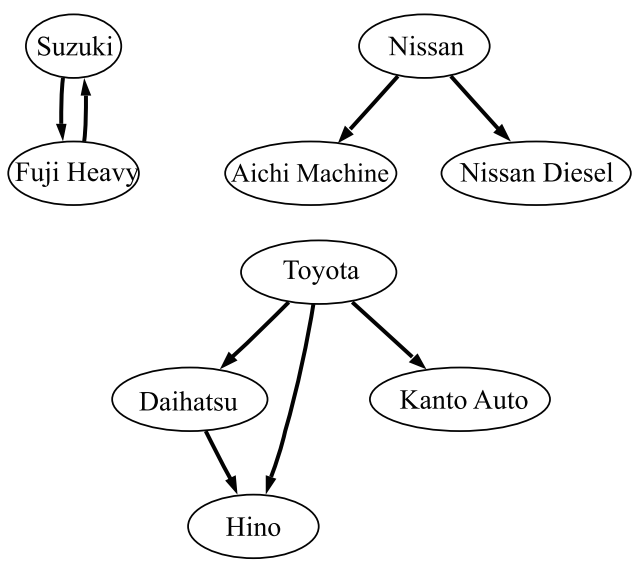

Figure 4.6: Shareholding network in automobile sector

mobile industry. The companies belonging to the automobile sector are 13 in number, namely Isuzu Motors, Suzuki Motor, Daihatsu Motor, Toyota Motor, Mazda Motor, Aichi Machine Industry, Kanto Auto Works, Mitsubishi Motors, Nissan Diesel Motor, Nissan Motor, Hino Motors, Fuji Heavy Industries, and Honda Motor.

The network shown in Figure 4.6 is constructed from only these companies, and is constructed from directed links, so is referred to as a directed graph or digraph. Sometimes we ignore the direction of links to simplify discussion, and, in addition, there are networks constructed from links which were not originally directed. Unsurprisingly, networks with undirected links are called undirected graphs.

Isuzu Motors, Mazda Motor, Mitsubishi Motors, and Honda Motor, do not appear in Figure 4.6, indicating that these companies are not connected to companies in the same industry by a shareholding relationship. In this 
case we used major shareholder data and stock holding data, and these data sources list only a limited number of companies. If a complete list of shareand stockholders were available there is a possibility that connections between these four companies and other companies belonging to automobile industry would be revealed. Figure 4.6 also shows that the network is constructed from three connected components. Suzuki Motor and Fuji Heavy Industries are connected by a bidirectional link meaning that there is a cross-shareholding. In addition, both Toyota Motor and Daihatsu Motor are shareholders of Hino Motors.

\subsubsection{The Interlocking Directors' Network}

The members of the board of directors of a company are selected at the meeting of shareholders, and their names then announced to the public, along with any interlocking directors. However, the previous history of members of a corporate board is not necessarily announced to the public. However, for listed companies in 2004, we use data which contains lists of interlocking directors and the previous history of members of the corporate board. ${ }^{8}$

By employing this data we can conceptually draw an interlocking directors' network and corporate board network as shown in Figure 4.7. This network is constructed from two kinds of nodes, namely directors and corporate boards. A network constructed in this way from two kinds of nodes is called a bipartite graph. If a certain director links many corporate boards and if the director's ideas for the management are frequently adopted, the director's effect is obviously widespread, and so it is natural to consider that such boards are strongly connected by the director. Acting on this consideration we get the upper right panel of Figure 4.7, which is constructed from just one type of node, i.e., corporate boards. The process by which we reduce a network with two types of node to that with only one type is called graph reduction.

Conversely, if we consider that the directors on the same corporate board know each other, we get the lower right panel of Figure 4.7, which is solely constructed from directors. Both are interesting, but in this study we will not investigate the director network, and will instead focus on the company (corporate board) network.

Since we can identify interlocking directors and the previous history of directors we can trace links from the previous board of a company to its present board, and from the main corporate board to sub-corporate boards, and then draw these networks as directed graphs.

If we draw the corporate board network alongside the shareholding network shown in Figure 4.6, we get Figure 4.8, with the dotted lines indicating newly drawn links. As before all the thirteen automobile companies do not appear in this figure, indicating that some companies are not connected in this way with other companies in the same industry. In this figure, the number beside each dotted link corresponds to the number of interlocking directors

\footnotetext{
${ }^{8}$ This data is commercially available from Toyo Keizai Inc.
} 


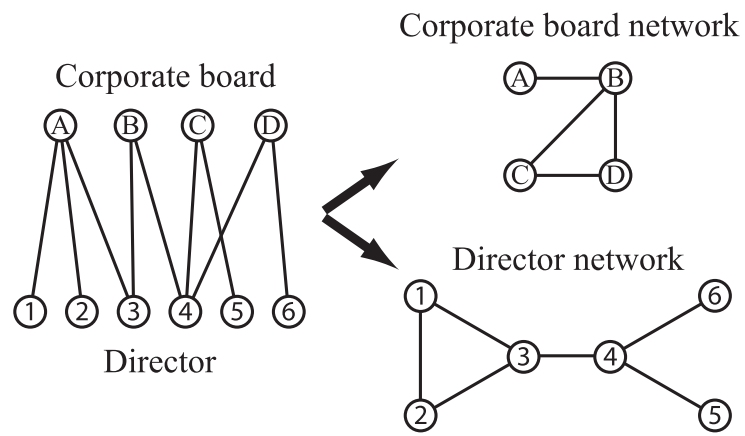

Figure 4.7: The corporate board and directors' network and its reduced graphs

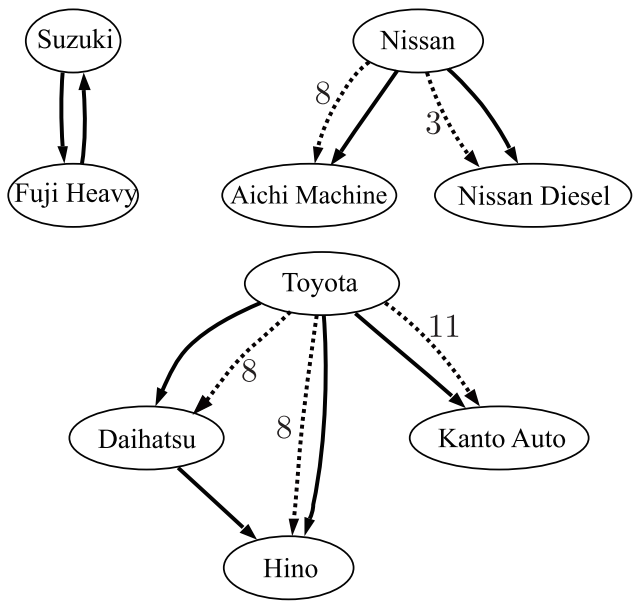

Figure 4.8: The corporate board network in automobile industry

and the number of directors connected by a relationship of previous history. For example, in the case of Daihatsu Motor, eight directors are sent from the board of Toyota Motor or were previously members of the board of Toyota Motor. As with the shareholding network, this network is constructed from three connected components, including the group clustered around Toyota Motor and the group centred on Nissan Motor. 


\subsubsection{The Transaction Network}

When we talk about friends, we can probably easily and honestly answer the question "how many friends do you have?" However, if we are asked "Who are your friends?" we may hesitate, and, even if we attempt an answer, vanity might tempt us to include the name of some well-known person. Something similar happens when a company answers the question "Which companies are your business partners?". In general, a company does not have an obligation to disclose the name of business partners, so business data companies get information of suppliers and customers by conducting an interview, and because of the vanity effect the resulting data is considered to be potentially biased.

Nevertheless, we can gain some insight from this data, and we use two kinds to investigate what we will call the transaction network. One source gives business partner information for 1,404 listed companies, which are nonfinancial companies listed in the first section of the Tokyo Stock Exchange in $2004 .^{9}$ The total number of links in this dataset is 55,348 , and therefore each of the 1,404 companies has approximately 80 links. The other gives data for all listed companies in Japan in 2004 and this data lists the five main suppliers and the five main customers for each company. ${ }^{10}$ In our analysis below we synthesise these two dataset, and consider the resulting transaction network.

To draw this network we trace links along the flow of materials and services (which is the reverse of the flow of money), and thus we get part of the network shown in Figure 4.5 for all listed companies, just as we did for the shareholding network. In the case of a transaction network the flow of materials and services defines the direction of the links. Hence, the white circles on the left side of Figure 4.5 correspond to main suppliers, which are located upstream of physical distribution. On the other hand, white circles on the right of Figure 4.5 correspond to main customers, which are located downstream of physical distribution. The transaction network is constructed by connecting these parts.

We will once again focus on the case of the automobile industry, and draw the transaction network on shareholding and interlocking directors' networks shown in Figure 4.8, thus leading to Figure 4.9. Thin and dashed lines are newly added links, and the direction of these links corresponds to the direction of physical distribution. As was the case with the shareholding network and

\footnotetext{
${ }^{9}$ This data is provided by Teikoku Data Bank Ltd.

${ }^{10}$ This data is provided by Nikkei NEEDS.
}

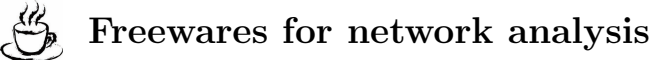

Graph drawing and graph analysis need considerable programming skills, so we might expect that software for graph analysis and graph drawing is expensive, but in fact there is a great deal of free software, for example, Graphviz, Pajek and Network Workbench. 


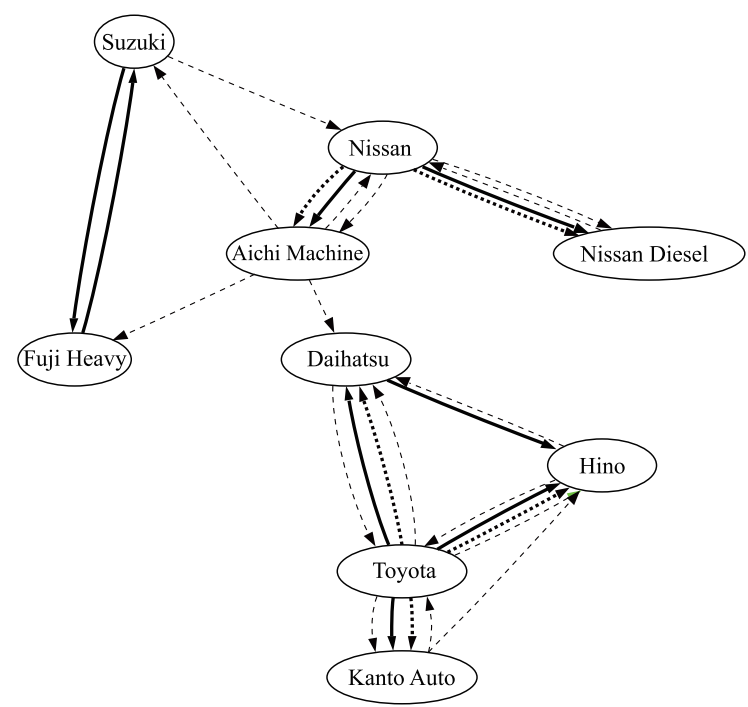

Figure 4.9: Transaction network of the automobile industry

interlocking directors' network, four companies, Isuzu Motors, Mazda Motor, Mitsubishi Motors, and Honda Motor, are not in the transaction network shown in Figure 4.9. The shareholding and interlocking directors' networks are separated into three connected components as shown in Figure 4.8. However, in this case, these connected components are connected into one component as shown in Figure 4.9.

In Figure 4.9, we can see that there are two groups. One is the group centred on Toyota Motor, and the other is the group centred on Nissan Motor. However, these two groups are connected by the link running from Aichi Machine Industry to Daihatsu Motor. In addition, the links from Aichi Machine Industry to Suzuki Motor and Fuji Heavy Industries play an important part in making one connected component. In this case, Aichi Machine Industry connects different groups, and is called a gatekeeper.

In most offices there are well-informed people who may not hold a senior post but play an important role in connecting the various parts of the department. Such a person is a gatekeeper. The importance of gatekeepers is characterised by betweenness centrality. This index is explained in Section 4.4.4. In Figure 4.9, we can see that there are many overlaps between shareholding links, the interlocking directors' network, and the links of the transaction network. 


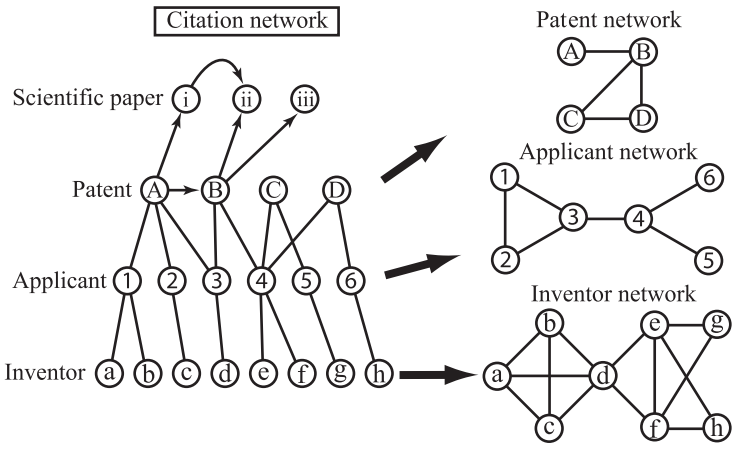

Figure 4.10: Patent network and its reduced graphs

\subsubsection{The Innovation Network}

The patent office publishes information relating to patent applications, and there are several databases derived from that information. For our work we have chosen to investigate cumulative patent data up to 2004. This set contains many items for each patent, for example, citation of scientific papers and patents, main text, classification of technology, applicants, inventors, address of applicants and inventors, etc. Such information is conceptually drawn in the left panel of Figure 4.10. Based on this figure we can construct many kinds of networks.

For example, as shown in Figure 4.10, if we use the citation of scientific papers and patents we can construct a network in which scientific papers and patents or technologies are nodes. In addition, if we extract keywords from the main text and consider them in relation to the citation network, we can construct a conceptual network in which keywords are nodes.

As conceptually shown in Figure 4.10, we can see that patent "A" and patent "B" are connected by applicant " 3 ". In addition, we can also see that patents "B", "C", and "D" are connected by applicant " 4 ". Thus, as shown in the upper right panel of Figure 4.10, we can construct a network with patents as nodes. Conversely, applicants " 1 ", "2", and " 3 " are connected by patent "A", and applicants " 3 " and " 4 " are connected by patent "B". Hence, as shown in the centre right panel of Figure 4.10, we can construct a network with applicants as nodes. In many cases, applicants correspond to companies, therefore, we can regard this network as having companies as nodes. In the current study we call this a network of joint patent applications. In addition, we can say that inventors "a", "b", "c", and "d" are connected by patent "A", and inventors "d", "e", and "f" are connected by patent "B". Thus, as shown in the lower right panel of Figure 4.10, we can construct a network with inventors as nodes.

Just as we did before, we will restrict our analysis to the case of the 
automobile industry. However, and this is a significant difference, the links in a patent network do not have direction, therefore it is drawn as undirected network. If we draw the patent network alongside that for shareholding and interlocking directors, we obtain Figure 4.11. Undirected and dashed lines represent the connections of joint applications for patents. The number beside each link corresponds to the number of joint application for patents up to 2004. However, this number suffers from inaccuracy because it is very difficult to identify companies with complete confidence. Different companies sometimes have the same name.

Contrary to the previous examples, this network is constructed from all thirteen companies, and although the Toyota Motor and the Nissan Motor groups were evident in the previous networks they are not evident in Figure 4.11. An interesting point is that Toyota Motor and Nissan Motor are not connected directly, but are connected by an intermediary, Honda Motor. We can also see that there are many overlaps between the links of the shareholding and interlocking directors' network and those of joint patent application network. In addition, companies, which are not connected in the shareholding and interlocking directors' network, are connected in this case. From this fact, we can infer that for the purposes of innovation companies are sometimes connected beyond company walls.

We can also investigate the network of joint patent applications constructed from companies belonging to the automobile (automobile) and electric machinery (automobile-related) sectors. In this case we obtain Figure 4.12. Although there are many companies the remarkable fact is that there are many joint patent applications between Toyota Motor and Denso Corporation, with the total number amounting to 915. There are also many joint patent applications between Honda Motor and Stanley Electric, with the total amounting to 114 . On the other hand, there are no joint patent applications between Nissan Motor and companies belonging to the electric machinery (automobile-related) category. Thus, we can infer that Nissan Motor applies for patents with companies belonging to the electric machinery industry, not the electric machinery (automobile-related) industry. However, as noted before, there is possibility that there are missing links, because patent data is not necessarily complete.

\subsection{Network Indices}

At this point we will turn to a discussion of the characteristics of networks through the calculation of network indices. Up to now, we have considered four types of networks, i.e., the shareholding network, the interlocking directors' network, the transaction network, and the innovation network. As shown above, some companies are connected to other companies with multiple relationships, i.e., multiple links (multilinks). A network with multiple links can be called a multiple network or a multiple graph (multigraph).

Now suppose that the weight of every link is equal to 1 . Thus, if two nodes 


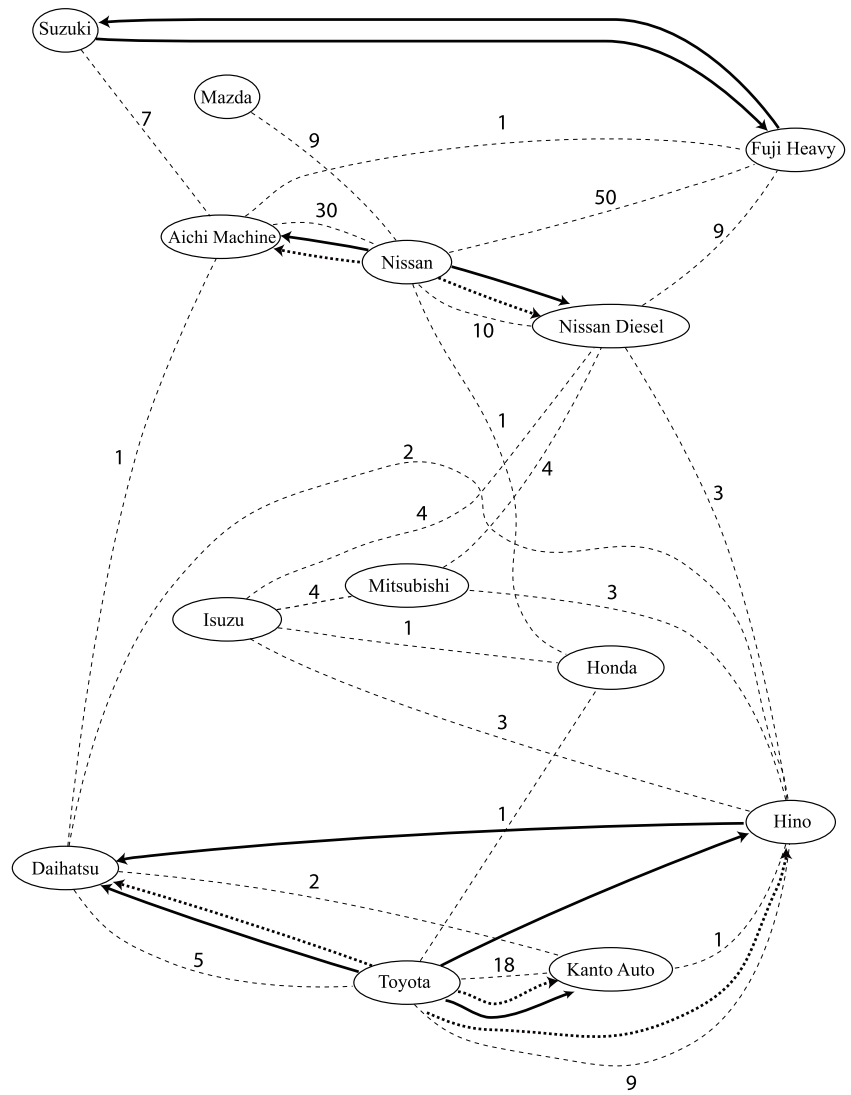

Figure 4.11: Network of joint application of patent in automobile industry 


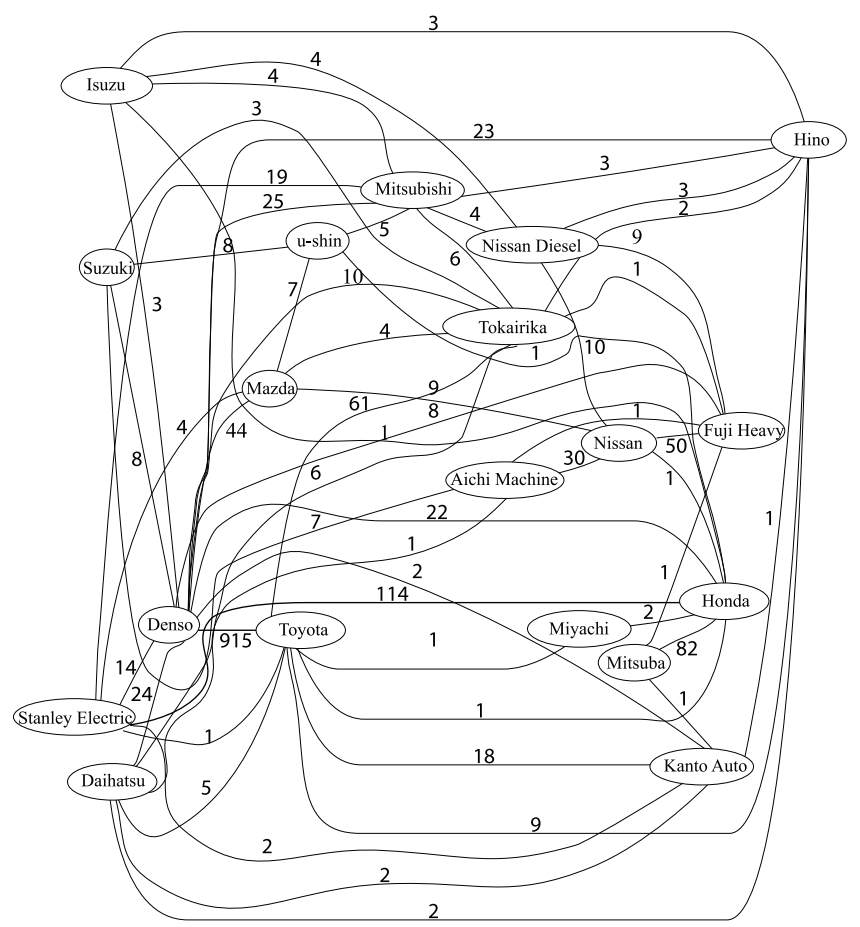

Figure 4.12: Network of joint patent applications between automobile and electric machinery industries 


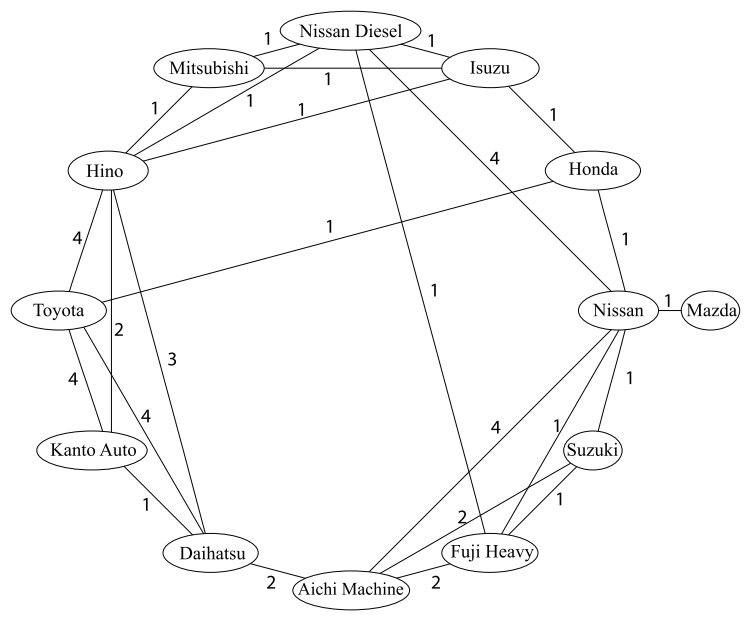

Figure 4.13: Weighted network of automobile industry

are connected by four types of relationship, we say that these two nodes are connected by one link with weight 4 . As explained here, a multiple network is regarded as a weighted network. The multiple network and weighted network are here given as an undirected network because a network of joint patent applications is an undirected network. In the light of this consideration the automobile industry network is drawn as Figure 4.13. The number beside each link represents its weight, and in what follows we will investigate this network by applying the methods of network analysis.

\subsubsection{Degree Centrality}

As stated before, the number of links held by a node is called its degree. In social network analysis in sociology degree is referred to as degree centrality. In this book, we denote the degree of a node $i$ as $k_{i}$, where $i$ corresponds to the company's number in the first row of Table. 4.1. The second row of Table. 4.1 corresponds to the name of the company. The degree calculated for each node of the network shown in Figure 4.13 corresponds to the third row of Table. 4.1. From this result we can see that Nissan Motor and Hino Motors have the largest degree value: $k_{10}=k_{11}=6$. On the other hand, Mazda Motor has the smallest value: $k_{5}=1$.

The sum weight of links attached to each company, which is called the node strength or the vertex strength, is summarised in the forth row of Table. 4.1. If we denote the weight of a link between node $i$ and node $j$ as 
Table 4.1: Network indices for 13 automobile companies

\begin{tabular}{|c|c|r|r|r|r|r|r|}
\hline Number & Name & $k_{i}$ & $s_{i}$ & $L_{i}$ & $C_{i}$ & $C_{i}^{\mathrm{w}}$ & $C_{i}^{\mathrm{b}}$ \\
\hline \hline 1 & Isuzu & 4 & 4 & 1.92 & 0.5 & 0.5 & 0.03 \\
\hline 2 & Suzuki & 3 & 4 & 2.17 & 1 & 1 & 0 \\
\hline 3 & Daihatsu & 4 & 10 & 1.75 & 0.5 & 0.53 & 0.11 \\
\hline 4 & Toyota & 4 & 13 & 1.92 & 0.5 & 0.62 & 0.05 \\
\hline 5 & Mazda & 1 & 1 & 2.5 & 0 & 0 & 0 \\
\hline 6 & Aich Machine & 4 & 10 & 1.83 & 0.5 & 0.53 & 0.111 \\
\hline 7 & Kanto Auto & 3 & 7 & 2.17 & 1 & 1 & 0 \\
\hline 8 & Mitsubishi & 3 & 3 & 2 & 1 & 1 & 0 \\
\hline 9 & Nissan Diesel & 5 & 8 & 1.58 & 0.4 & 0.34 & 0.20 \\
\hline 10 & Nissan & 6 & 12 & 1.58 & 0.27 & 0.28 & 0.28 \\
\hline 11 & Hino & 6 & 12 & 1.67 & 0.4 & 0.4 & 0.16 \\
\hline 12 & Fuji Heavy & 4 & 5 & 1.83 & 0.67 & 0.67 & 0.04 \\
\hline 13 & Honda & 3 & 3 & 1.75 & 0 & 0 & 0.09 \\
\hline
\end{tabular}

$w_{i j}$, the node strength of node $i$ is defined by

$$
s_{i}:=\sum_{j \in \operatorname{adj}(i)} w_{i j}
$$

Here, $\operatorname{adj}(i)$ represents the set of adjacent nodes of node $i$. Thus, $j \in \operatorname{adj}(i)$ states that "node $j$ belongs to the set of adjacent nodes of node $i$ ". By summing up $w_{i j}$ for $j$, we obtain $s_{i}$. In the case of the network shown in Figure 4.13, Toyota Motor has the largest value for node strength: $s_{4}=13$. On the other hand, Mazda Motor has the smallest value for node strength, $s_{5}=1$, just as it had for degree value. A consideration of both node strength and degree yields a deep understanding of the characteristics of a network.

\subsubsection{Shortest Path Length}

As noted before, path length is also one of elementary quantities characterising a network. We will here consider the path length in Figure 4.13 in concrete terms. For example, let's take Toyota Motor as the starting point. The company is connected to four companies by one link, these four companies being Hino Motors, Honda Motor, Daihatsu Motor, and Kanto Auto Works. In addition, Toyota Motor is connected to five companies by two links, these five companies being Mitsubishi Motors, Nissan Diesel Motor, Isuzu Motors, Nissan Motor, and Aichi Machine Industry. Fuji Heavy Industries, Mazda Motor, and Suzuki Motor are connected to Toyota Motor by three links. Thus, the average value of links necessary to connect Toyota Motor and the other companies is given by $L_{4}=(1 \times 4+2 \times 5+3 \times 3) / 12=1.92$. If we calculate $L_{i}$ for each company, the result is summarised in the fifth row in Table. 4.1. From this result, Nissan Diesel Motor and Nissan Motor have the smallest value for shortest path length. In other words, these two companies can easily approach 
other companies. On the other hand, Mazda Motor has the largest value for shortest path length. Finally, if we calculate averaged path length for this network, we obtain $\langle L\rangle=1.897$.

\subsubsection{Clustering Coefficient}

As explained previously, the clustering coefficient is an index which quantifies the cliqueyness of nodes. Taking the network shown in Figure 4.13 we can calculate a concrete clustering coefficient. As in the case of path length, we will consider the case of Toyota Motor as an example. Toyota Motor has four links, i.e., companies. Thus, the possible number of combinations to connect these four companies to each other is equal to the number of ways of selecting two companies from four companies, i.e., $C(4,2)=4(4-1) / 2=6$. However, as we can see in Figure 4.13, three sets are realised. These are Hino Motors and Daihatsu Motor, Hino Motors and Kanto Auto Works, and Kanto Auto Works and Daihatsu Motor. The clustering coefficient is defined by the ratio of realised numbers of combinations to possible numbers of combinations; therefore, in the case of Toyota Motor, the clustering coefficient is given by $C_{4}=3 / 6=0.5$.

As we can see in Figure 4.13, Mazda Motor has one link; therefore, the possible number of combinations is $C(1,2)=0$. Hence, the clustering coefficient of Mazda Motor diverges. As stated before, the clustering coefficient of a node with one link is defined by 0 . If we calculate the clustering coefficient for each company, the result is summarised in the sixth row in Table. 4.1. By definition, the clustering coefficient has value from 0 to 1 . As stated above, the clustering coefficient of Mazda Motor is equal to 0 by definition, and although Honda Motor has three links, the clustering coefficient of Honda Motor is also equal to 0. However, the clustering coefficient of Suzuki Motor, Kanto Auto Works, and Mitsubishi Motors is equal to 1, because all possible combinations are realised. If we calculate the averaged value of clustering coefficient of the network, we obtain $\langle C\rangle=0.518$. This value is relatively large.

A weighted clustering coefficient, that is to say, a clustering coefficient defined by considering the weight of links, has also been proposed. There are several definitions of weighted clustering coefficient, some of them using the weight of links connecting to a node (see Saramäki et al. (2007) for example). Taking definition of the node strength in Section 4.4.1, we denote the weight of a link between node $i$ and node $j$ as $w_{i j}$. In addition, we denote node strength and the degree of the node $i$ as $s_{i}$ and $k_{i}$, respectively. Thus, if node $j$ and node $k$, which are adjacent to node $i$, are connected with each other, we define the weighted clustering coefficient as

$$
C_{i}^{\mathrm{w}}=\frac{1}{s_{i}\left(k_{i}-1\right)} \sum_{j, k \in \operatorname{adj}(i)} \frac{w_{i j}+w_{i k}}{2},
$$

which was originally proposed in Barrat et al. (2004). If the weights are equal this expression reduces to the previously stated formula for the case without weight, as is easily verified. 
By using this definition of weighted clustering coefficient we obtain the result summarised in the seventh row of Table. 4.1. The weighted clustering coefficient $C_{i}^{\mathrm{w}}$ has a value between 0 and 1 , just as it did in the case of the clustering coefficient $C_{i}$. Now, we can compare $C_{i}$ and $C_{i}^{\mathrm{w}}$. As can easily be seen, if $C_{i}$ is equal to 0 or 1 , then $C_{i}^{\mathrm{w}}$ is also equal to 0 or 1 . On the other hand, if $C_{i}$ has a value between 0 and 1 , even if two nodes have the same value of $C_{i}$, these two nodes sometimes have a different value of $C_{i}^{\mathrm{w}}$. For example, as shown in Table. 4.1, Isuzu Motors, Daihatsu Motor, Toyota Motor, and Aichi Machine Industry have $C_{1}=C_{3}=C_{4}=C_{6}=0.5$. However, by considering a weighted clustering coefficient, we obtain $C_{4}^{\mathrm{w}}>C_{3}^{\mathrm{w}}=C_{6}^{\mathrm{w}}>C_{1}^{\mathrm{w}}$. In other words, Toyota Motor is responsible for the most tightly connected cluster.

\subsubsection{The Betweenness Centrality of Nodes}

We can now consider an experiment in which we randomly select two nodes from a network and connect these two nodes by the shortest path. If we repeat this experiment many times, we notice that some nodes and links appear frequently on the shortest path, and others never do so. This characteristic of nodes and links can be quantified with a betweenness centrality.

Let us start with nodes and denote the number of the shortest paths connecting node $i$ and node $j$ as $\sigma_{j, k}$. Note that we do not consider the length of the shortest path. In addition, we denote the number of shortest paths, which passes through node $i$ and connects node $j$ and node $k$, as $\sigma_{j, k}(i)$. The betweenness centrality of node $i$ is defined by

$$
C_{i}^{\mathrm{b}}:=A \sum_{i \neq j \neq k \in V} \frac{\sigma_{j, k}(i)}{\sigma_{j, k}} .
$$

Note that $A$ is a normalisation factor and $V$ is a set of nodes in network.

For example, we can consider the case connecting Mazda Motor and Kanto Auto Works in Figure 4.13, where we find that the length of the shortest path is equal to 4 , and that three paths exist.

- Mazda $\rightarrow$ Nissan $\rightarrow$ Nissan Diesel $\rightarrow$ Hino $\rightarrow$ Kanto Auto

- Mazda $\rightarrow$ Nissan $\rightarrow$ Honda $\rightarrow$ Toyota $\rightarrow$ Kanto Auto

- Mazda $\rightarrow$ Nissan $\rightarrow$ Aich Machine $\rightarrow$ Daihatsu $\rightarrow$ Kanto Auto

Thus, $\sigma_{5,7}=3$. In addition, $\sigma_{5,7}(10)=3$ for Nissan Motor, and $\sigma_{5,7}(i)=$ $1,(i=3,4,6,9,11,13)$ for Nissan Diesel Motor, Hino Motors, Honda Motor, Toyota Motor, Aichi Machine Industry, and Daihatsu Motor respectively. Thus, $C_{10}^{\mathrm{b}}=1$ for Nissan Motor, and $C_{i}^{\mathrm{b}}=1 / 3,(i=3,4,6,9,11,13)$ for the other five companies, when we ignore the normalisation. Though we are only considering the path between Mazda Motor and Kanto Auto Works, the betweenness centrality is obtained by calculating for every path of every combination of nodes. The eighth row of Table. 4.1 is the normalised betweenness centrality of each node. From this result we can see that Nissan Motor has 
the largest value. This is because we can see in Figure 4.13, all companies have to pass Nissan Motor to arrive at Mazda Motor.

\subsubsection{Cliques}

The clustering coefficient, which was explained earlier, is constructed by regarding a triangle as a cluster, when a subgraph constructs a triangle. However, we are not limited to triangles and can extend the concept to clusters larger than three nodes. For example, suppose there are four nodes and these four nodes are connected to each other. Such a network is extendable to a network including many nodes, in which all nodes are connected to each other. When every node is completely connected to every other we refer to it as a complete graph. In addition, such a complete graph is also referred to as an $\boldsymbol{n}$-clique, where $n$ is the number of nodes contained in the network.

As we can see in Figure 4.13, there are three 4-cliques. These are summarised in Table. 4.2, where we can observe that the clique constructed from Hino Motors, Mitsubishi Motors, Nissan Diesel Motor, and Isuzu Motors is regarded as the truck making group. In addition, the clique constructed from Hino Motors, Toyota Motor, Kanto Auto Works, and Daihatsu Motor is regarded as the Toyota group. These two cliques are connected by Hino Motors, so we can conclude that Hino Motors is the gatekeeper. The other clique is constructed from Nissan Motor, Aichi Machine Industry, Fuji Heavy Industries, and Suzuki Motor, and is regarded as the Nissan group. As we can see in Figure 4.13 there are no cliques constructed from five or more nodes.

If we calculate the total sum of weight of links within each clique, (the result is summarised in the third row of Table. 4.2) we observe that the Toyota group has the largest value for the total sum of link weights, and we can infer that the Toyota group is a more tightly connected group than other groups.

\subsection{Statistical Properties of Network Indices}

In the previous section, as an example, we investigated a small network constructed only from automobile companies, and calculated some indices and considered the overall characteristics of the network. In this section, we will investigate some large networks and consider the statistical characteristics of network indices.

\subsubsection{Comparison of Industries by Using Network In- dices}

Before we calculate network indices, as explained in the previous section, it should be noted that if the number of nodes becomes large then disconnected parts may appear. If we regard these disconnected parts as islands, the whole network looks like a world map. That is, although each node in an island is connected, there are no links connecting the islands. Consequently, we must 
Table 4.2: Clique of 13 companies belonging to the automobile industry

\begin{tabular}{|c|l|c|}
\hline Clique & Company & Total sum of weight \\
\hline \hline \multirow{5}{*}{ 4-clique } & Hino & 6 \\
& Mitsubishi & \\
& Nissan Diesel & \\
& Isuzu & \\
\cline { 2 - 3 } & Hino & \\
& Toyota & \\
& Kanto Auto & \\
& Daihatsu & \\
\cline { 2 - 3 } & Nissan & \\
& Aichi Machine & \\
& Fuji Heavy & \\
& Suzuki & \\
\hline 5-clique & none & \\
\hline
\end{tabular}

calculate network indices for the largest island, which we call the largest connected component.

Firstly, we will investigate the whole network described so far. As shown in the second row of Table. 4.3, this network contains $N=3,576$ nodes and $K=55,332$ links. This network constructs one connected component, i.e., the largest connected component is the network itself. In addition, the averaged degree, the averaged path length, the averaged clustering coefficient, and the averaged weighted clustering coefficient are $\langle k\rangle=30.95,\langle L\rangle=2.78$, $\langle C\rangle=0.24$, and $\left\langle C^{\mathrm{w}}\right\rangle=0.19$, respectively. If we calculate betweenness centrality $C^{\mathrm{b}}$ for each node, we can list the top five companies, namely Japanese Securities Finance Co. Ltd., Mitsui \& Co. Ltd., Mitsubishi Corporation, Hitachi Ltd., and Panasonic Corporation, as shown in the second row of Table. 4.3. ${ }^{11}$ The first company, i.e., Japanese Securities Finance Co., Ltd., has a large number of links concerned with shareholding; therefore it has large value of betweenness centrality. The second and third companies, i.e., Mitsui \& Co., Ltd. and Mitsubishi Corporation, have many links concerned with shareholding and transactions. The forth and fifth companies, i.e., Hitachi Ltd., and Panasonic Corporation, have many links concerned with shareholding, transactions, and innovation.

The analysed results for the electric machinery industry are summarised in the third row of Table. 4.3. This industry contains 285 companies and 2,196 links. In this case the largest connected component is also the network itself. However, if we compare $\langle k\rangle$ of this industry with that for whole network, we find that the $\langle k\rangle$ of the electric machinery industry is approximately half that of whole network. However, the value of $\langle L\rangle$ is almost the same in both the electric machinery industry and whole network. On the other hand, $\langle C\rangle$ and

\footnotetext{
${ }^{11}$ Note that banks were not included in this investigation.
} 
Table 4.3: Network indices for the whole network, electric machinery, and automobile

\begin{tabular}{|c|c|c|c|c|}
\hline \multicolumn{2}{|c|}{ index } & Whole network & Electric machinery & Automobile \\
\hline \multicolumn{2}{|c|}{$N$} & 3,576 & 285 & 85 \\
\hline \multicolumn{2}{|c|}{$K$} & 55,332 & 2,196 & 568 \\
\hline \multicolumn{2}{|c|}{$\langle k\rangle$} & 30.95 & 15.41 & 13.37 \\
\hline \multicolumn{2}{|c|}{$\langle L\rangle$} & 2.78 & 2.32 & 1.98 \\
\hline \multicolumn{2}{|c|}{$\langle C\rangle$} & 0.24 & 0.65 & 0.89 \\
\hline \multicolumn{2}{|c|}{$\left\langle C^{\mathrm{w}}\right\rangle$} & 0.19 & 0.45 & 0.46 \\
\hline \multirow{5}{*}{$C^{\mathrm{b}}$} & 1 & Japanese Securities Finance & Hitachi & Honda \\
\hline & 2 & Mitsui \& Co. & Toshiba & Nissan \\
\hline & 3 & Mitsubishi & Panasonic & Suzuki \\
\hline & 4 & Hitachi & Mitsubishi Electric & Toyota \\
\hline & 5 & Panasonic & Sharp & Fuji Heavy \\
\hline
\end{tabular}

$\left\langle C^{\mathrm{w}}\right\rangle$ for the electric machinery industry is larger than that of whole network, meaning that companies in electric machinery industry are densely connected with each other.

The analysed results for the automobile industry are summarised in the fourth row of Table. 4.3. This industry contains the electric machinery (automobilerelated), automobile (automobile), automobile (auto body and others), and automobile (auto parts) categories. The network for this industry contains 87 nodes and 569 links, and is constructed from two connected components, the largest connected component containing 85 nodes and 568 links. In the largest connected component the averaged degree is $\langle k\rangle=13.37$, which is almost the same as the averaged degree in the electric machinery industry. On the other hand, the averaged shortest path length $\langle L\rangle$ in this industry is smaller than that in electric machinery industry. Thus, companies in the automobile industry are connected by a smaller number of steps than companies in the electric machinery industry. In addition, though the clustering coefficient $\langle C\rangle$ of the automobile industry is larger than that of the electric machinery industry, the weighted clustering coefficient $\left\langle C^{\mathrm{w}}\right\rangle$ is almost the same as that in the electric machinery industry.

We have now compared the group of whole network with those in the electric machinery and automobile industries. However, the numbers of nodes and links in these industries are very different, so let us compare the pharmaceuticals and steel industries, which have almost the same number of nodes and links, as shown in the second and third rows of Table 4.4. By comparing these two industries we can find that the values of $\langle C\rangle$ and $\left\langle C^{\mathrm{w}}\right\rangle$ are very different, namely, the pharmaceuticals industry has very small values compared to the value calculated for whole network. On the other hand, the values of $\langle C\rangle$ and $\left\langle C^{\mathrm{w}}\right\rangle$ in the steel industry are much larger than that in the pharmaceuticals industry. From this fact we can infer that companies in the steel industry are densely and tightly connected to each other compared to companies in the 
Table 4.4: Network indices of the pharmaceuticals and steel industries

\begin{tabular}{|c|c|c|c|}
\hline \multicolumn{2}{|c|}{ index } & Pharmaceuticals & Steel \\
\hline \multicolumn{2}{|c|}{$\bar{N} N$} & 49 & 47 \\
\hline \multicolumn{2}{|c|}{$K$} & 126 & 119 \\
\hline \multicolumn{2}{|c|}{$\langle k\rangle$} & 5.14 & 5.06 \\
\hline \multicolumn{2}{|c|}{$\langle L\rangle$} & 2.53 & 2.30 \\
\hline \multicolumn{2}{|c|}{$\langle C\rangle$} & 0.22 & 0.57 \\
\hline \multicolumn{2}{|c|}{$\left\langle C^{\mathrm{w}}\right\rangle$} & 0.16 & 0.42 \\
\hline \multirow{5}{*}{$C^{\mathrm{b}}$} & 1 & Mitsubishi Pharma & Nippon Steel \\
\hline & 2 & Takeda Pharmaceutical & Sumitomo Metal \\
\hline & 3 & Seikagaku & Kobe Steel \\
\hline & 4 & Yamanouchi Pharmaceutical & Hitachi Metals \\
\hline & 5 & Tanabeseiyaku & Daido Steel \\
\hline
\end{tabular}

pharmaceuticals industry.

\subsubsection{Degree Distribution}

As stated in Section 2.1.1, the coverage of data is important when considering degree distribution. Though this book concentrates on investigation of the shareholding network, the interlocking directors' network, the transaction network, and the innovation network, it is not necessarily possible to investigate the precise degree distribution for these networks. For example, in the case of the transaction network the data contains only the main suppliers and customers and this coverage of data is insufficient. However, we can examine the shareholding and innovation networks because data for these networks satisfies the coverage requirement.

As explained in Section 4.3.1, the data useful to an investigation of the shareholding network are major shareholder and stock holdings information. Major shareholder data contains the total number of shareholders (the total number of incoming degrees) and the list of the top thirty major shareholders (incoming links) for each listed company. In addition, stock holding data contains the total number of stocks (the total number of outgoing degrees) and the list of the top ten major investing companies (outgoing links) for each listed company.

We will now turn to an investigation of the distribution of outgoing degree in the shareholding network. As stated above, because of the limitation of stock holdings data we cannot know precisely which listed companies are connected to outgoing links, but we can to some extent determine the total number of outgoing links. On the other hand if we use major shareholder data we can find companies with large outgoing degrees, even for unlisted companies, because, companies with large outgoing degrees appear frequently in the major shareholder data. Thus we can approximately determine that the 
number of times a company appears in the major shareholder data corresponds to the number of outgoing degrees of unlisted companies.

We will use the total number of outgoing degrees, which is listed in stock holdings data, since this number is a precise value. The black circles in Figure 4.14 correspond to the degree distribution of companies contained in this data. The abscissa corresponds to degree and the ordinate corresponds to rank. This figure is log-log plot of the rank-size plot, which is explained in Section 2.1.2. Figure 4.14 is also kind of rank-size plot, however, we will simply call it the degree distribution. In addition, for unlisted companies we can consider the number of appearances in major shareholder data as being approximately equal to the value of outgoing degrees. The open squares in Figure 4.14 correspond to the degree distribution obtained by the method stated here. Thus, unification of these two types of outgoing degree distributions derives the outgoing degree distribution with some precision. However, some companies appear in both outgoing degree distributions so we will adopt a larger value as the true outgoing degree. The crosses in Figure 4.14 are obtained by the method explained here, and the solid line and dashed line correspond to power-law distributions with power-law exponents $\gamma=1.5$ and 2.0 , respectively. In network science if we denote degree as $k$ and consider the degree distribution, the power-law exponent $\gamma$ is generally represented by using a probability density function:

$$
p(k)=A k^{-\gamma},
$$

where $A$ is a normalisation constant. In short, although $\gamma$ is the same as $\alpha$ as defined in Section 2.1.2, in network science it is customary to denote it as $\gamma$. From this figure, we can see that shareholding network is approximately a scale-free network because the tail part of the outgoing degree distribution follows a power-law distribution.

On the other hand, for the innovation network, i.e., a network of joint patent applications, we can investigate a database that covers all joint patent applications up until 2002, and so analyse the degree distribution. This is the TamadaDB, which contains all information relating to 4,998,464 patents applied for in Japan over the period 1993 to 2002. The largest connected component of the network of joint patent applications constructed from TamadaDB contains 34,840 nodes and 84,840 links. The degree distribution of this largest connected component is shown in Figure 4.15. ${ }^{12}$ This figure is a log-log plot of degree distribution, and the abscissa corresponds to degree and the ordinate corresponds to rank. The solid line in this figure is a power-law distribution with a power-law exponent equal to 2 . We can see in this figure that the distribution follows the power-law distribution in a wide range of degrees; therefore, the network of joint patent applications is a scale-free network.

\footnotetext{
${ }^{12}$ This result is obtained by collaboration with Prof. S. Tamada (Kwansei Gakuin Univ.) and Dr. H. Inoue (Osaka Sangyo Univ.). (W.S)
} 


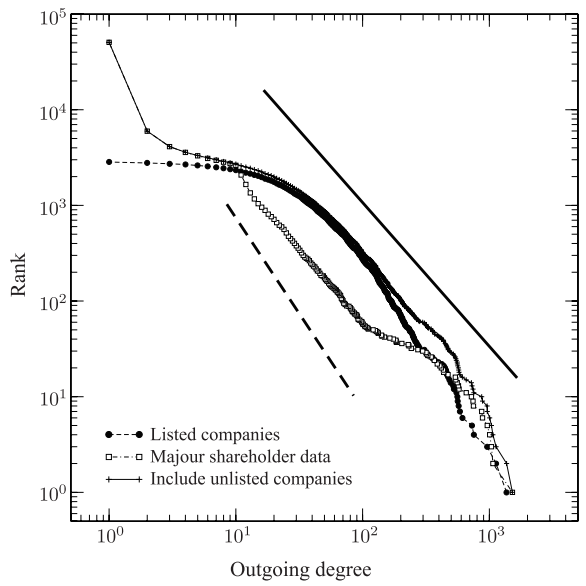

Figure 4.14: Outgoing degree distribution of the shareholding network

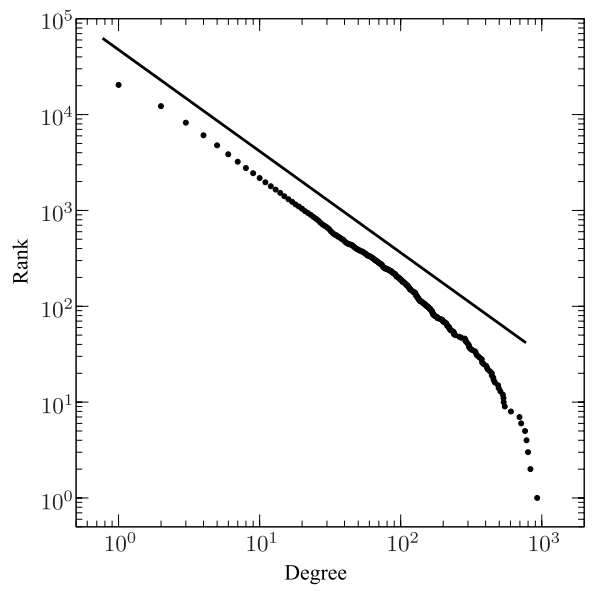

Figure 4.15: Degree distribution of the network of joint patent application 


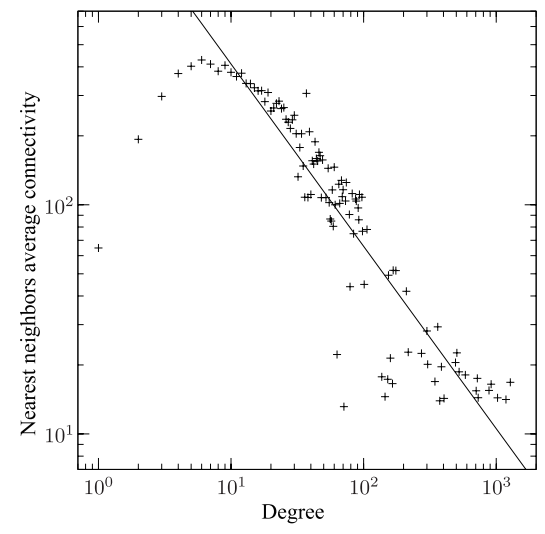

Figure 4.16: Degree correlation of the shareholding network

\subsubsection{Correlation related to Degree}

At this stage we can consider the correlation related to degree. We assume that the node with degree $k^{\prime}$ is adjacent to the node with degree $k$. In addition, we define the conditional probability density $p_{c}\left(k^{\prime} \mid k\right)$, which is the probability of selecting nodes with degree $k^{\prime}$ when we select a node with degree $k$. Thus, the averaged degree of a node adjacent to a node with degree $k$ is given by

$$
\left\langle k_{\mathrm{nn}}\right\rangle=\sum_{k^{\prime}} k^{\prime} p_{c}\left(k^{\prime} \mid k\right)
$$

Here, $\left\langle k_{\mathrm{nn}}\right\rangle$ is called the nearest neighbour's average connectivity.

If we calculate $\left\langle k_{\mathrm{nn}}\right\rangle$ for the shareholding network in 2002 and plot it on a $\log$-log graph, we obtain Figure 4.16. The abscissa corresponds to $k$ and ordinate corresponds to $\left\langle k_{\mathrm{nn}}\right\rangle$. This figure displays degree-degree correlation. The solid line in Figure 4.16 is given by

$$
\left\langle k_{\mathrm{nn}}\right\rangle(k)=A k^{-\nu}, \quad \nu \simeq 0.8,
$$

where $A$ is a proportionality coefficient. This result shows that the averaged degree of nodes adjacent to the node with a large degree is small. In short, the probability of connecting between hubs is very low.

It is known that the degree-degree correlation in the study of gene networks and the World Wide Web is represented by a power-law function with an exponent of $\nu \simeq 0.5$ (Pastor-Satorras et al., 2001). Thus, the degree-degree correlation of the shareholding network is almost the same as that of the gene network and the World Wide Web. 


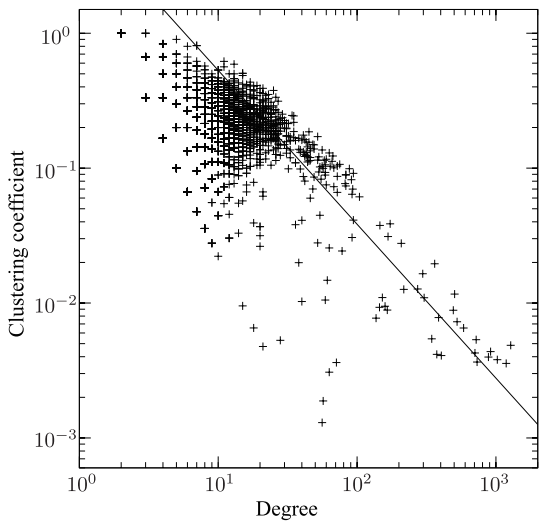

Figure 4.17: Correlation between the degree and clustering coefficients in the shareholding network

In a friendship network, we know experimentally that the probability of connecting persons with many friends is very high. In short, there is a high probability of connecting hubs in a human network. In such a case the scatter plot is not similar to Figure 4.16, in which distribution is diagonally right upwards, but is diagonally right downwards, which is opposite to the case of the shareholding network, the gene network, and the World Wide Web.

We will next consider the correlation between the degree and clustering coefficients of each node. If we investigate the case of the shareholding network in 2002, we obtain the result shown in Figure 4.17. In this figure, the abscissa corresponds to degree $k$ and the ordinate corresponds to the clustering coefficient $C$. The solid line in this figure is given by

$$
C(k)=A^{\prime} k^{-\alpha}, \quad \alpha \simeq 1,
$$

where $A^{\prime}$ is the proportionality coefficient. From this figure, we can see that nodes with a large degree have a small clustering coefficient. However, we should note that the nodes with a large degree have many clusters. To calculate the clustering coefficient, the number of actual clusters is divided by the possible number of combinations of clusters, i.e., $C\left(k_{i}, 2\right)=k_{i}\left(k_{i}-1\right) / 2$. In the case of a node with large degree, $C\left(k_{i}, 2\right)=k_{i}\left(k_{i}-1\right) / 2$ is very large. Thus, the clustering coefficient of such a node is small.

It is also noted that for the gene network and the World Wide Web the nodes with a large degree have a small clustering coefficient (Ravasz et al., 2002). Thus, the shareholding network has similar characteristics to the gene network and the World Wide Web. To explain the relation between degree and clustering coefficients the concept of network motif and hierarchy have 


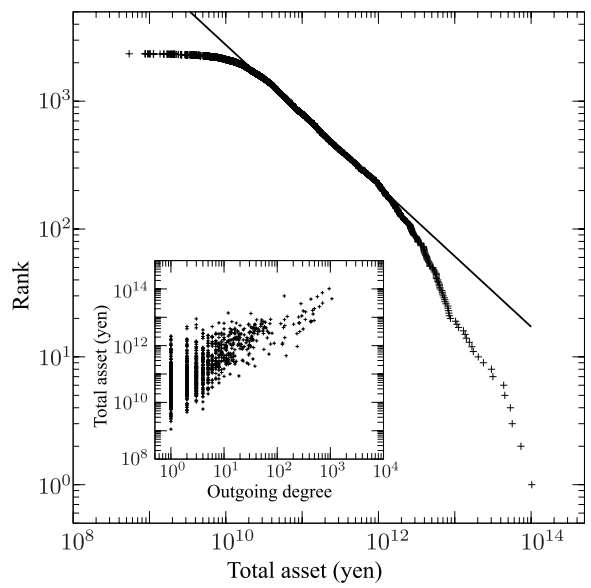

Figure 4.18: Distribution of total assets, and correlation between degree and total assets

been proposed (Barabási and Oltvai, 2004; Milo et al., 2002; Shen-Orr et al., 2002). Network motif is the minimal unit constructing a network, and has a typical shape. Some network motifs construct a module, and some modules construct another module. It is considered that such a hierarchical structure in a network realises the relation $C(k) \propto k^{-\alpha}$.

\subsubsection{Shareholding Network and Company Size}

In this section, we will construct a network by using only major shareholder data, and then calculate the outgoing degree. In addition, we will consider the correlation between outgoing degree and the total assets of a company and the correlation between outgoing degree and the company's age. Figure. 4.18 is a rank-size plot of total assets of listed companies at the end of March 2002. In this figure, the abscissa corresponds to total assets and the ordinate corresponds to rank. The solid line in this figure is a power-law distribution with an exponent of $\mu=0.6$. From this figure, we can see that total company assets follows an approximate power-law distribution.

At this point we are only considering listed companies at the end of March 2003. Thus, this data does not contain every company having total assets greater than some value, so this data does not cover the distribution of total assets in detail. Because of this defect the complete power-law shown in Chapter 2 does not appear in Figure 4.18. However, in Chapter 2, we have shown that the distribution of income, sales, and profit of Japanese companies follows a power-law distribution. In addition, we have also shown that the distribution of total assets and sales of European companies follows a power- 


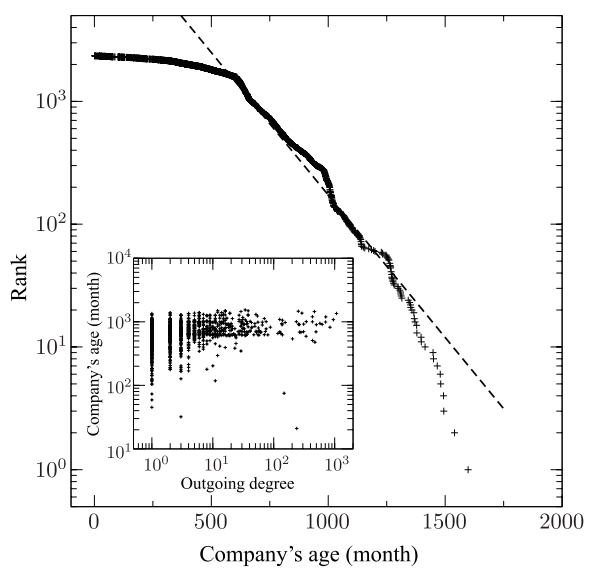

Figure 4.19: Distribution of company age, and correlation between outgoing degree and the age

law distribution. Thus, it is reasonable to assume that if we can obtain the complete list of total assets for Japanese companies, we can probably show that they also follow a power-law distribution in the wide range of total assets.

The small panel in Figure 4.18 is a log-log plot of the correlation between outgoing degree and the total assets of listed companies at the end of March 2002. From this figure, we can see that the outgoing degree and total assets have a strong and positive correlation. In fact, Kendall's $\tau$ (see Section 3.1.7) is equal to $\tau=0.53$.

In the present study we define a company's age by counting the months from its foundation day to the end of March 2002, the date of the company data we used. The semi-log plot of the rank distribution of company's age is shown in Figure 4.19. In this figure, the abscissa corresponds to company age and the ordinate corresponds to rank. The dashed line in this figure is an exponential distribution. From this figure, we can see that the distribution of a company's age follows an approximately exponential distribution. In Section 3.4.2, we discussed the life span of companies, and showed that the distribution of company life spans follows an exponential distribution. Thus, it is to be expected that company age and life span will follow the same distribution.

The small panel in Figure 4.19 shows the log-log plot of the correlation between outgoing degree and company age for listed companies at the end of March 2002. From this figure, we can see that outgoing degree and company age have a weak and positive correlation. In fact, Kendall's $\tau$ (see Section 3.1.7) is equal to $\tau=0.20$.

Map constructed by four mega banks and listed 


\section{companies in 2002}

So far we have drawn several networks, but we have not given any meaning to the positions of companies in those networks. The study of graph drawing has a long history, and international conferences on this subject are held every year. Elementary physics might be useful determining the relative positions of companies in the entire network of the shareholding relationship.

To do so, we first establish the initial positions of companies as shown below in this column. Note that banks and nonfinancial listed companies are randomly arranged in a two dimensional plane, with the four mega banks, Mizuho Bank, Sumitomo Mitsui Banking, The Bank of Tokyo-Mitsubishi, and UFJ Bank all marked as large filled circles in this figure. Our data lists the top ten major shareholders for each bank and each company. The list of the top ten major shareholders includes stock brokerage companies, trust companies, and insurance companies. However, we are interested in a network constructed from banks and nonfinancial listed companies, so we will ignore the other companies.

Let us suppose that a company "A" is owned by two shareholders, and that one shareholder has two shares and the other shareholder has one share. Now, we regard the number of shares as a mass, and set the appropriate position of company as the centre of mass. Thus in this example the appropriate position of company "A" is given by the point on which the line connecting two shareholders is divided by $2: 1$. However these two shareholders also have some shareholders; therefore, these two shareholders also move to an appropriate position. By repeating this calculation we can regard such a configuration as the appropriate state if we can obtain the stationary state of the distribution of banks and nonfinancial listed companies.

And in fact, if we actually perform this calculation we do obtain the stationary state. However, this state is not unique and depends on the initial state of the distribution of banks and nonfinancial listed companies. The typical stationary state is shown in the right figure of this column, and from this figure, we can see that most companies are owned by the four mega banks.
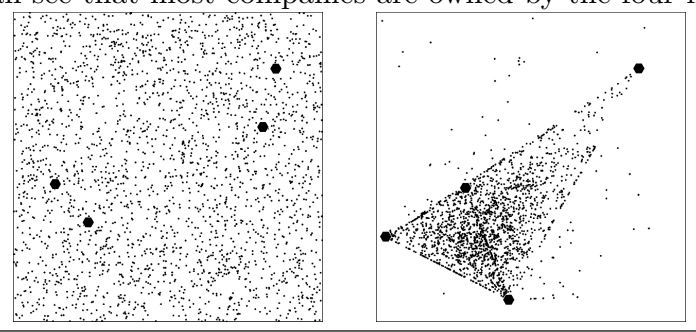

\subsection{Dynamics of the Company Network}

Up to this point we have considered networks as a snapshot of a certain year, thus we can regard these as static networks. In this section, we will 
consider change both in the network and the correlation between companies in networks; that is to say, we will investigate dynamic networks.

\subsubsection{Change in the Shareholding Network}

Patent data is extremely useful when discussing change in a network, since we can trace the development from the first patent. In this network a new patent application connects to a network constructed from patents previously applied for. This type of network is referred to as a growing network, that is to say one in which the number of nodes and links increases with time.

In many economic networks, growth and collapse of the network, and the rewiring of links, happens frequently. However, this never occurs in a patent network, so instead we will examine the shareholding network since changes are numerous and frequent. We will investigate a shareholding network constructed only from major shareholder data.

Figure. 4.20 is the sub-network extracted from the network constructed from listed companies in 1985. In this sub-network, shareholders are companies belonging to the transport machine industry. ${ }^{13}$ In this figure black nodes correspond to companies belonging to the transport machine industry, and white nodes correspond to companies belonging to other industries. We note that four companies, Toyota Motor, Nissan Motor, Honda Motor, and Mitsubishi Motors, are hubs in the network. In addition, we can see that these four hubs (companies) are not directly connected. As explained in Section 4.5.3, this sort of network means that the degree-degree correlation is negative. Hubs are connected by companies belonging to both the transport machine industry and another industry. These companies generally belong to the electric machinery industry or industries in which companies make parts of automobiles.

By applying the same criterion for the shareholding network in 1995, we obtain Figure 4.21. By comparing Figure 4.20 and Figure 4.21, we can see that there is no remarkable change. If we draw the same type of figure in 2000, we obtain Figure 4.22. This figure is almost same as Figure 4.20 and Figure 4.21. However, Figure 4.23 for 2003 is remarkably different from Figures 4.20, 4.21, and 4.22. In this figure, Mitsubishi Motors and Nissan Motor are no longer hubs in the network. In the period between 2000 and 2003, foreign capital participated in these two companies, and the management policy changed significantly, resulting in the drastic change observable in Figure 4.23.

To consider whether a similar change occurred or not in other industries we will investigate the case of the electric machinery industry. Figure. 4.24 is the sub-network extracted from the network constructed from listed companies in 1985. In this sub-network, shareholders are companies belonging to the electric machinery industry. Black nodes correspond to companies belonging to the electric machinery industry, and white nodes correspond to companies belonging to other industries. We can see that eight companies, Hitachi

\footnotetext{
${ }^{13}$ The transport machine industry includes the automobile industry and some other industries.
} 


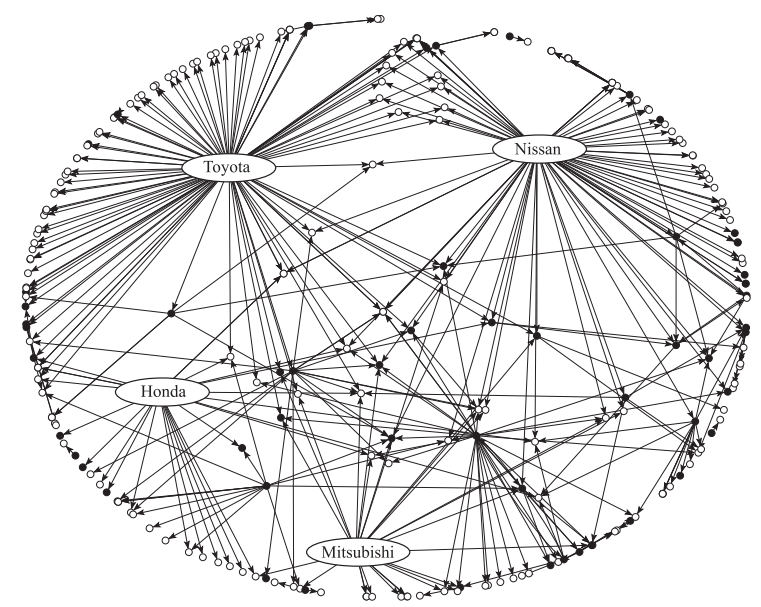

Figure 4.20: Shareholding network with shareholders belonging to the transport machine industry (1985)

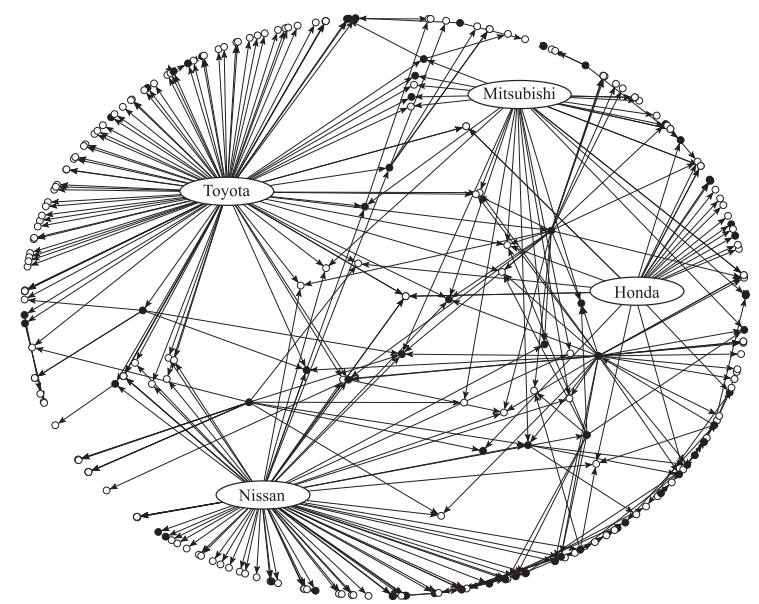

Figure 4.21: Shareholding network with shareholders belonging to the transport machine industry (1995) 


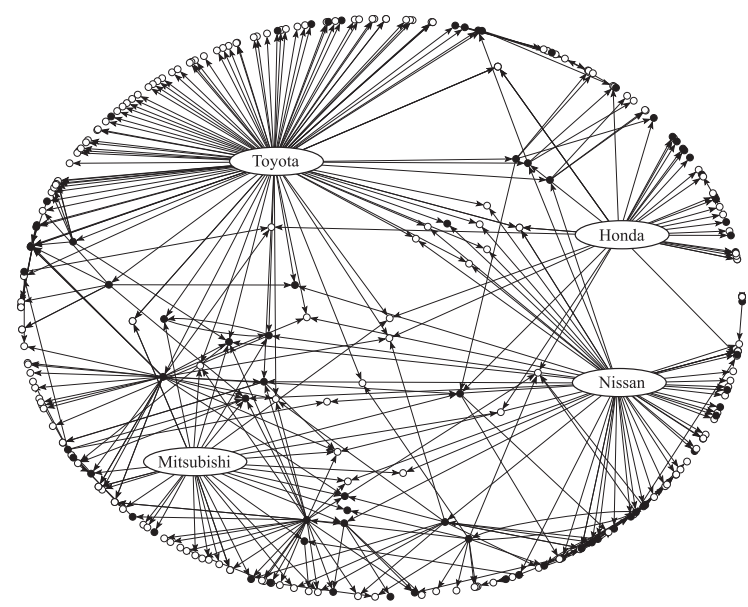

Figure 4.22: Shareholding network with shareholders belonging to the transport machine industry (2000)

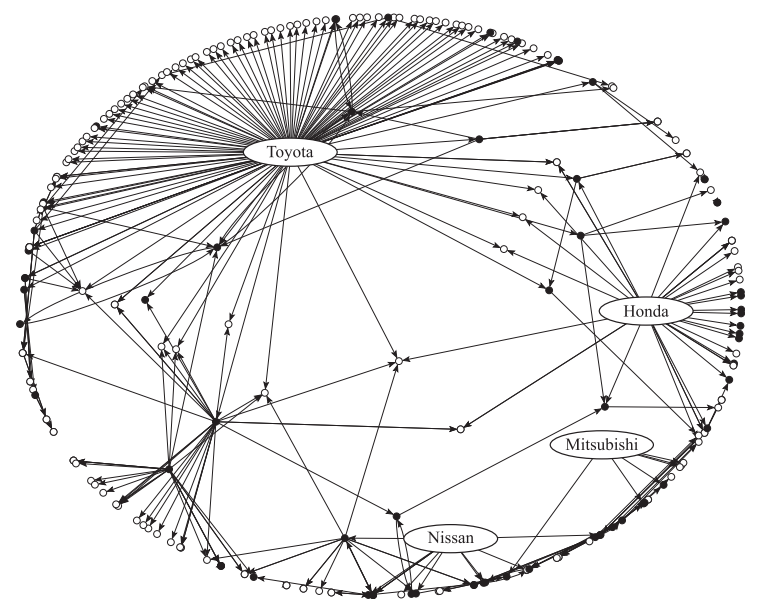

Figure 4.23: Shareholding network with shareholders belonging to the transport machine industry (2003) 


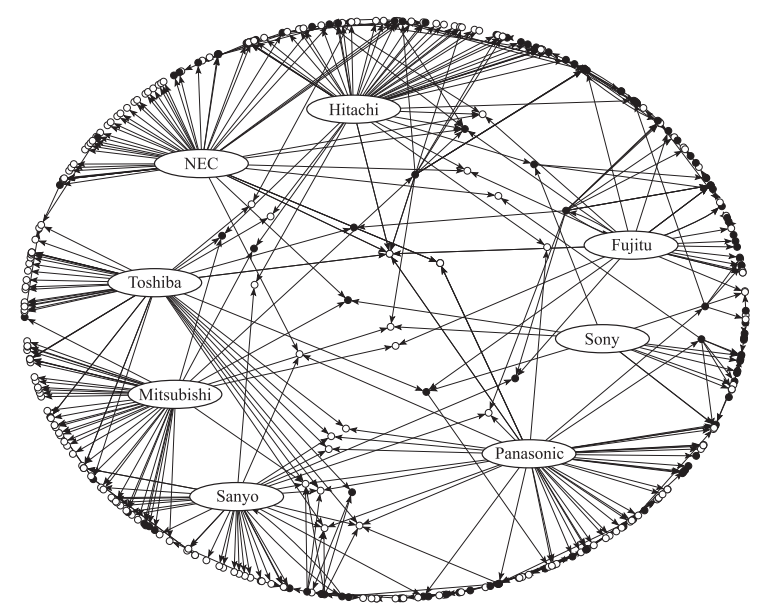

Figure 4.24: Shareholding network with shareholders belonging to the electric machinery industry (1985)

Ltd., NEC Corporation, Sony Corporation, Toshiba Corporation, Fujitu Ltd., Panasonic Corporation, Mitsubishi Electric Corporation, and Sanyo Electric Co. Ltd., are hubs in the network. As in the case of the transport machine industry, we can see that these eight hubs (companies) are not directly connected. As explained in Section 4.5.3, this nature of network means that the degree-degree correlation is negative.

As in the case of the transport machine industry, we can draw networks for 1995, 2000, and 2003 as Figures 4.25, 4.26, and 4.27, respectively. By comparing these four figures for the electric machinery industry, we can see that there is no remarkable change, and in fact there has been no drastic change of management, such as participation of foreign capital, in the electric machinery industry.

\subsubsection{Change of Degree Distribution}

We will now consider the change of degree distribution by using only major shareholder data. Originally the shareholding network was drawn as a directed graph. However, as explained in Section 4.3.1, the major shareholder data provides the list of the top thirty major shareholders for each listed company. Thus, if we draw the shareholding network by tracing links from shareholders to listed companies, the incoming degree has an upper bound. However, the outgoing degree does not have an upper bound. Thus, in this section, we will investigate only the outgoing degree.

Denoting the total number of nodes and links as $N$ and $K$ respectively, the changes of $N$ and $K$ are summarised in Table 4.5. From this table, we can 


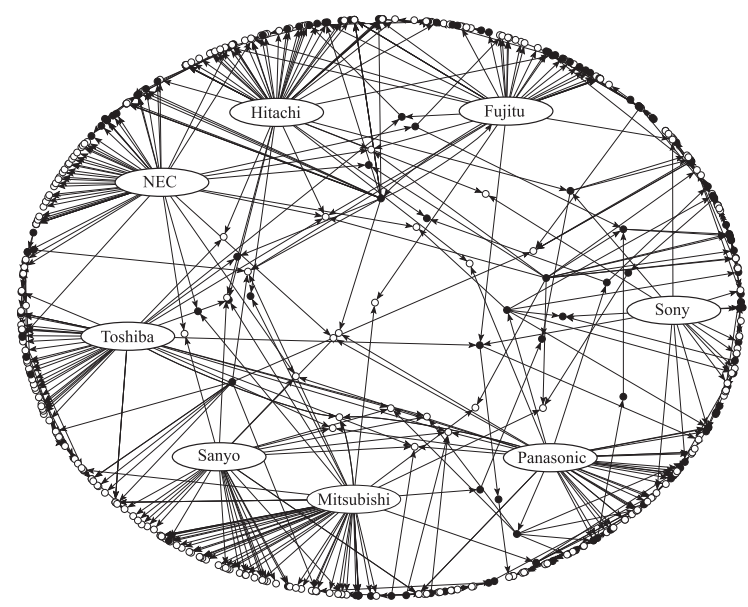

Figure 4.25: Shareholding network with shareholders belonging to the electric machinery industry (1995)

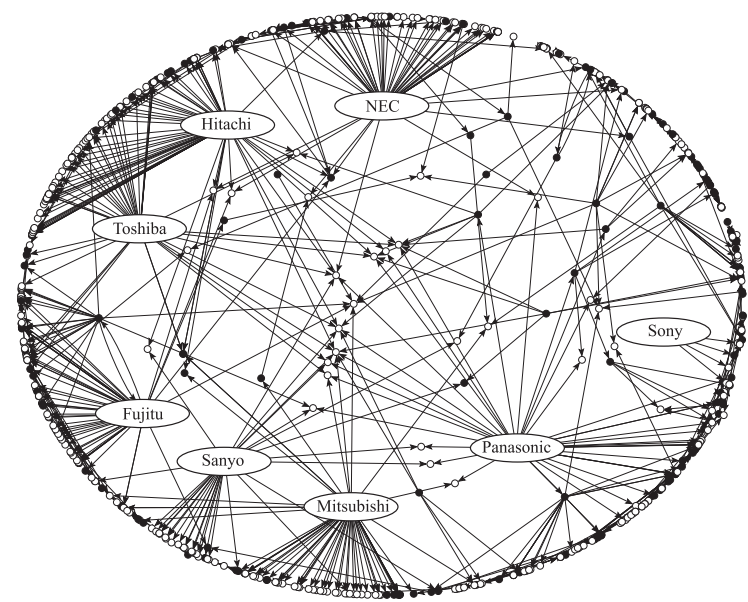

Figure 4.26: Shareholding network with shareholders belonging to the electric machinery industry (2000) 


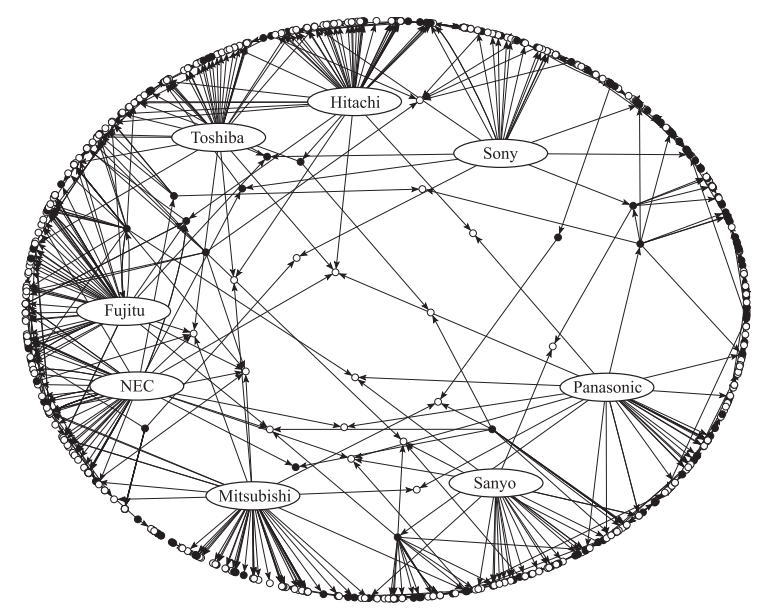

Figure 4.27: Shareholding network with shareholders belonging to the electric machinery industry (2003)

Table 4.5: Change of shareholding network

\begin{tabular}{|c||c|c|c|c|c|c|}
\hline year & 1985 & 1990 & 1995 & 2000 & 2002 & 2003 \\
\hline \hline$N$ & 2,078 & 2,466 & 3,006 & 3,527 & 3,727 & 3,770 \\
\hline$K$ & 23,916 & 29,054 & 33,860 & 32,586 & 30,000 & 26,407 \\
\hline$\gamma$ & 1.68 & 1.67 & 1.72 & 1.77 & 1.82 & 1.86 \\
\hline
\end{tabular}

see that $N$ increased rapidly between 1985 to 2002 and is slowly increasing in recent years. This means that the increase of the number of newly listed companies is slow. On the other hand, $K$ peaks in 1995, and decreases rapidly after 1995. This behavior of $K$ is related to bad-debt disposal after the year 1990, when the so-called Heisei bubble collapsed.

Figure 4.28 is a log-log plot of the cumulative distribution of outgoing degrees in $1985,1990,1995,2000,2002$, and 2003. In this figure, the abscissa corresponds to the outgoing degree and ordinate corresponds to the cumulative distribution. From this figure, we can see that all annual distributions can be well fitted by a linear function in the wide range of the distribution, though not in the tail part. In short, the distribution of outgoing degree follows a power-law distribution, and the shareholding network is a scale-free network. The change of the power-law exponent $\gamma$ is summarised in Table. 4.5. From this Table, we can see that $\gamma=1.67 \sim 1.86$.

An oligopoly of degree makes the power-law exponent $\gamma$ small. This characteristic of $\gamma$ is the same as the Pareto exponent discussed in Section 2.2. The 


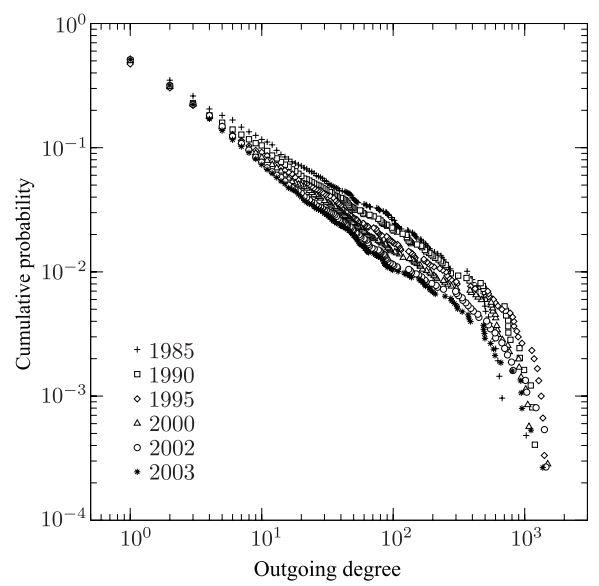

Figure 4.28: Change of degree distribution

change of $\gamma$ is summarised in Table. 4.5. As explained in Section 4.5.2, $\gamma$ is obtained by fitting the probability density function of degree with a power-law distribution. From this Table, we can see that $\gamma$ decreases slightly over the period 1985 to 1990 , and increases consistently after 1990. This means that shareholding became an oligopoly up to the Heisei bubble, and this oligopoly was eliminated after the collapse of Heisei bubble.

Now, we can consider why the power-law exponent changed. Some years ago, the bad-debt disposal of major banks was a serious problem in Japan. To settle bad-debts, banks and non-financial companies sold a portion of their stock holdings. It is believed that these stock holdings were not held by banks and companies for investment, but for cross-shareholding. Thus, it is to be expected that the change of power-law exponent has a relation with the cross-shareholding rate, i.e., the long-term shareholding rate. Long-term shareholding is not for speculative buying and selling, frequently within one year, but for stable possession for periods greater than a year.

We can now investigate the correlation between the power-law exponent and the shareholding rate with the unit base (long-term shareholding rate and cross-shareholding rate), as reported by Nippon Life Insurance (NLI) Research Institute. The left panel of Figure 4.29 shows the change of the long-term shareholding rate and the cross-shareholding rate. In this figure, filled circles and filled squares correspond to the long-term shareholding rate, and the cross-shareholding rate, respectively. In addition, the right panel of Figure 4.29 shows the correlation between the power-law exponent and longterm shareholding rate and the correlation between the exponent and crossshareholding rate. From this figure, we can see that power-law exponent has a strong and negative correlation with the long-term shareholding rate and 


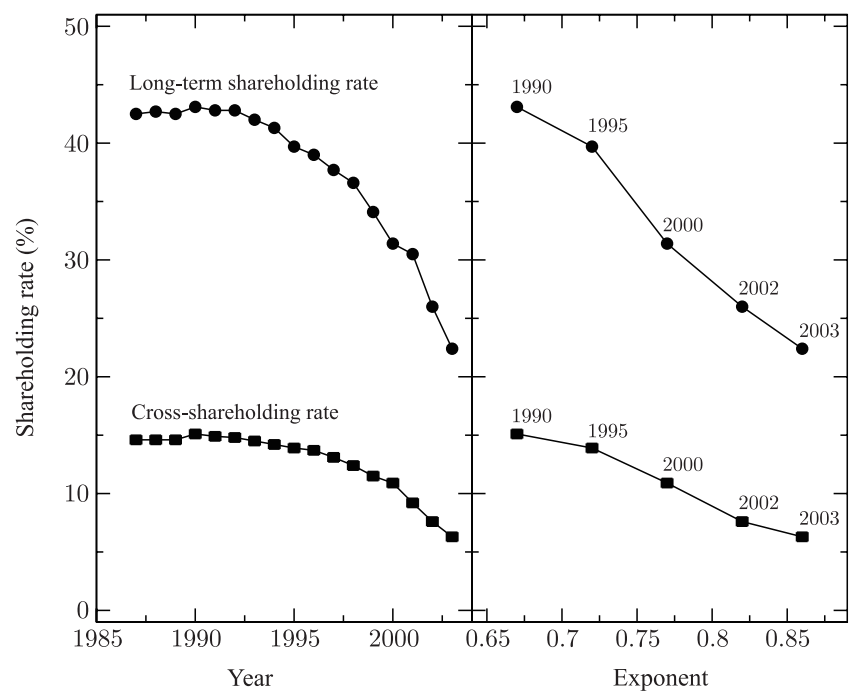

Figure 4.29: Change of the long-term shareholding rate and the cross-shareholding rate, and correlation with the power-law exponent

the cross-shareholding rate.

The degree distribution of the shareholding network has also been investigated for companies listed on Milano Italia Borsa (MIB), the New York Stock Exchange (NYSE), and the National Association of Security Dealers Automated Quotations (NASDAQ) (Garlaschelli et al., 2005). These analyses showed that the degree distribution follows the power-law distribution, exactly as is the case in Japan. For example, the power-law exponents of MIB, NYSE, and NASDAQ are $\gamma=1.97, \gamma=1.37$, and $\gamma=1.22$, respectively. These results are almost identical to those in Japan.

Thus, it is to be expected that a degree distribution following a powerlaw distribution is a universal structural feature of shareholding networks, although the value of power-law exponent $\gamma$ depends on country and year. In addition, if we consider that scale-free networks are found in many networks from biology to internet, it is reasonable to conclude that the shareholding network in Japan is not special.

In both degree distribution and also many power-law distribution phenomena the tail part of the distribution does not follow a power-law. In such cases the deviation from a power-law is sometimes ignored, because the tail part describes rare events. Nevertheless detailed investigation is promising, and we will examine this a little further.

If we plot the distribution of outgoing degree in the semi-log graph, we can obtain Figure 4.30, and from this figure we can see that the tail part of the 


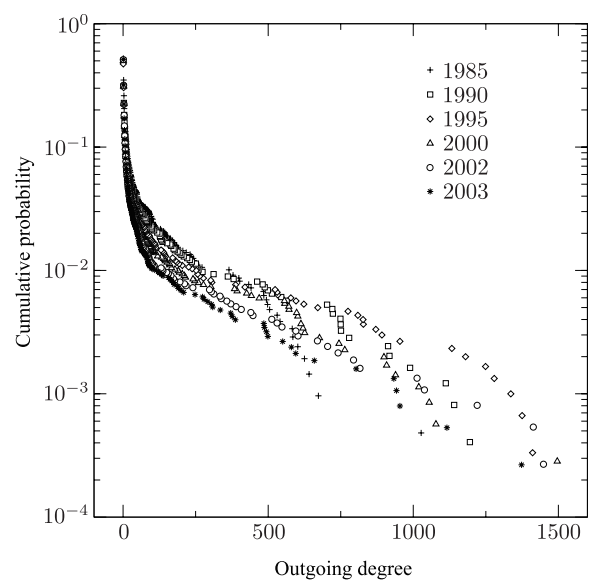

Figure 4.30: Change of degree distribution in a shareholding network

distribution is well fitted by a linear function, namely, the tail part follows an exponential distribution. In addition, this tail part is mainly constructed from financial companies. On the other hand, $95 \%$ of the part following a power-law distribution is constructed from nonfinancial companies. Thus, it is expected that the development mechanism of a network of financial companies differs from that for nonfinancial companies.

\subsubsection{Correlation between Companies in Networks}

In Section 4.6.1 and Section 4.6.2, we considered the change of network topology and the change of degree distribution as the dynamics of a network. However, we can take a different viewpoint and consider the correlation between companies in networks. For example we can consider the correlation between companies by using a correlation coefficient (see column in pp. 121).

A correlation coefficient has a value from -1 to 1 , thus when the correlation coefficient is close to 1 , it is said "strong and positive correlation", while when the correlation coefficient is close to -1 , it is said to be a "strong and negative correlation". In addition, when the correlation coefficient is close to 0 , there is said to be "no correlation". However, we should be aware that the correlation coefficient is on the ordinal scale, which represents the relative strength of the correlation. In short, when we compare the correlation coefficients 0.1 and 0.5 , we must not conclude that the correlation of the latter is five times stronger than the former.

When considering the correlation between companies in networks, we begin by imagining the correlation between sales and cost in the transaction network. 
In our analysis up to now we have investigated certain kinds of network, such as shareholding networks and transaction networks, so it is natural to wonder which network accounts for the strong correlation between companies. In addition, from the viewpoint of risk management, the correlation between bankruptcy probabilities is extremely interesting as it is connected with chainbankruptcy.

To investigate the correlation between companies, we need financial and network data for several years. However, for the transaction network we only have data for 2004, so we will assume that the shape (topology) of the transaction network in 2004 was sustained in the past. Hence, we will investigate the correlation between companies by assuming that companies change dynamically in a static network.

\section{Correlation between Growth Rates of Sales and Cost}

For an adequate description of the growth of each company, we must consider the direct interaction between companies through transactions, and we can check this by analysing data about growth of company (Ikeda et al., 2008).

Figure 4.31 shows the distribution of growth of sales $X$ and the distribution of growth of costs for $Y$ Japanese companies listed on the 1st Section of the Tokyo Stock Exchange in 2003. Cost is here defined as approximately equal to the cost of raw materials. In addition, growth rate is defined as the ratio of realised value in one year to that of the previous year. From Figure 4.31, we can see that the distribution of growth rate has a fat tail on both sides of the distribution, and follows a Laplace distribution (double-sided exponential distribution).

Now, we can anticipate that sales and costs (costs of raw materials) for a pair of companies with transactions will have a strong correlation. Thus, to check this expectation we will compare the correlation coefficient between the growth rate of sales and costs for pairs of companies linked by transactions and pairs of companies without such connections. The important thing to note is that we are not considering the correlation between sales and costs itself, but investigating the correlation between the growth rate of sales and the growth rate of costs. Because a correlation coefficient has meaning for stationary process, so sales and costs, which are generally considered to be a nonstationary process, must be rendered stationary by calculating the growth rate.

Figure 4.32 shows the correlation coefficient between the growth rate of sales and the growth rate of costs. From this figure, we can see that the distribution for pairs of companies with transactions shifts in the direction of a large correlation coefficient, compared to the distribution for pairs of companies without transactions. Although we can find large correlation coefficients for pairs of companies without transactions, we conclude that such a large correlation coefficient is an effect of the correlation with a business partner's business partner. It follows from this that in the discussion of the growth rate of each company we must consider the direct interaction between companies 

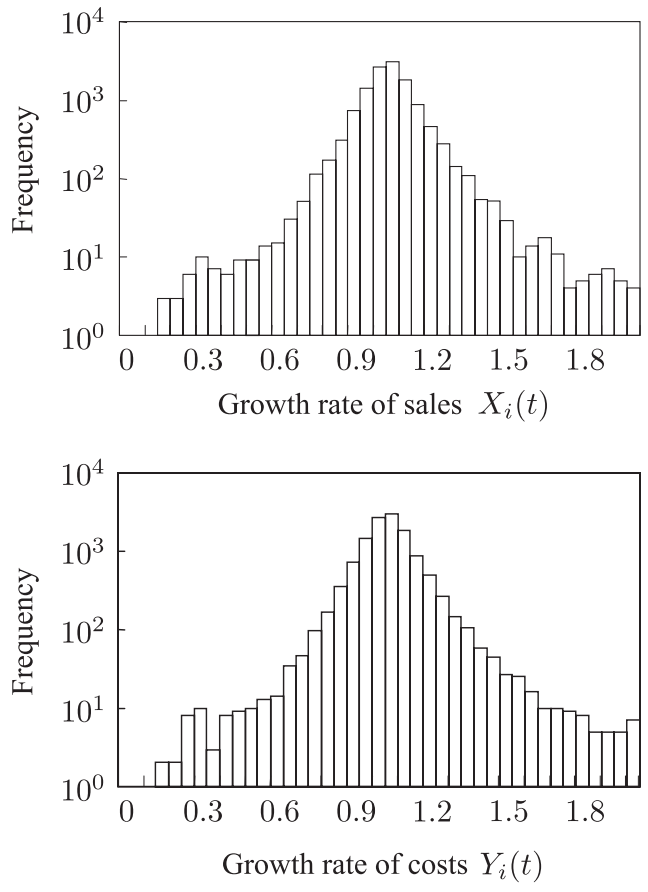

Figure 4.31: Distribution of growth rate of sales $X$ and that of costs $Y$ 


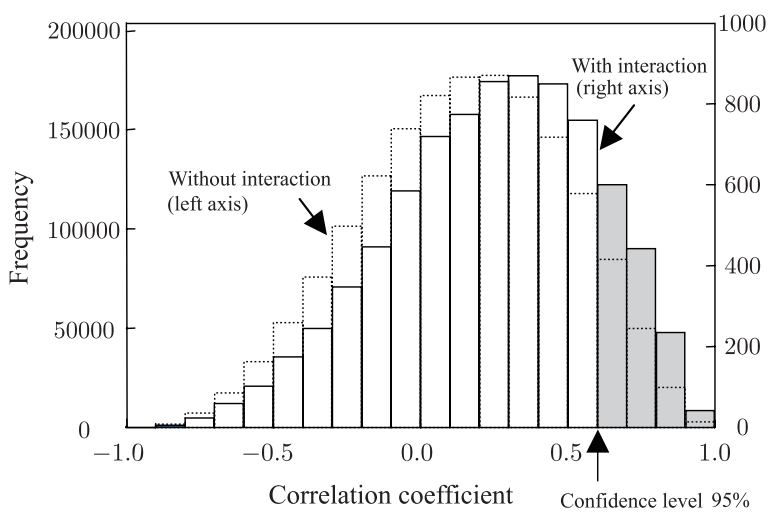

Figure 4.32: Correlation coefficient between growth rates for sales and costs

with transactional relationships. In the discussion of growth rate stated before we ignored the interaction between companies. Thus, in the next stage we will consider cases including interaction between companies.

In Figure 4.32, we find that even though we are investigating the correlation coefficient of growth rate between transacting companies there is an element showing negative correlation. It is reasonable to regard this negative part as noise, and thus, following basic statistical practise, we remove noise by using the noncorrelation test. The noncorrelation test is performed as follows: firstly we assume the null hypothesis that two statistical variables are uncorrelated with each other. If noncorrelation is realised the statistical quantity $T=r \sqrt{L-2} / \sqrt{1-r^{2}}$, which is obtained by using the correlation coefficient $r$ calculated from time series data, is characterised by the $t$ distribution with $L-2$ degrees of freedom. Here, $L$ is the length of time series. In addition, $P$ is the area larger than the statistical quantity $T$ in the $t$ distribution with $L-2$ degrees of freedom. In this case, the null hypothesis is rejected with confidence level $(1-P) \times 100 \%$. In Figure 4.32 , the shaded part shows that the correlation coefficient is statistically meaningful with a confidence level larger than $95 \%$.

The transaction network is drawn as a directed graph; therefore, we can calculate the incoming degree $k_{\text {in }}$ and outgoing degree $k_{\text {out }}$. If we set the direction of links as the direction of physical distribution, the incoming degree corresponds to the number of suppliers and the outgoing degree corresponds to the number of customers. Figure. 4.33 shows the cumulative probability distribution of incoming degree and outgoing degree, as defined above. The tail part follows an approximate power-law distribution. When we remove noise by using the noncorrelation test, the power-law exponent $\gamma$ is approx- 

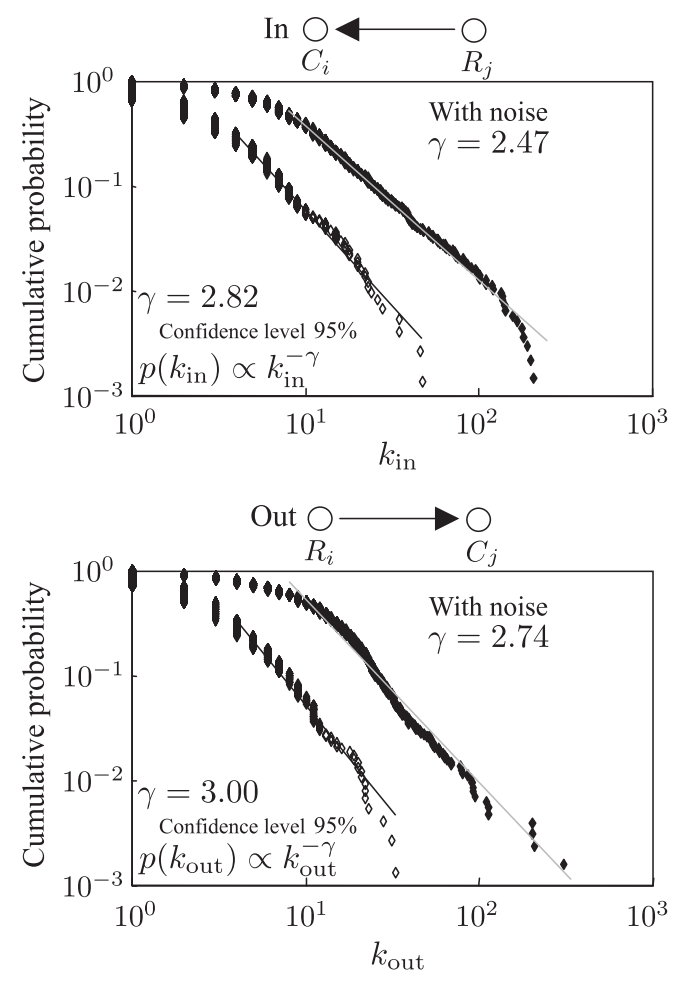

Figure 4.33: Cumulative probability distributions of incoming degree and outgoing degree 
imately equal to 3 , which is same value derived from the growing network model proposed by Barabási and Albert (Barabási and Albert, 1999).

If we consider the suppliers in the transaction network, the growth rate of cost for $i$-th company is represented by

$$
\delta Y_{i}(t+1)=\sum_{j=1}^{N_{s}} k_{i j} \delta X_{j}(t)+\epsilon_{i}(t),
$$

where $\delta X_{i}(t)$ and $\delta Y_{i}(t)$ are the normalised growth rate of sales $\left(X_{i}(t)-\right.$ $\left.\left\langle X_{i}\right\rangle_{t}\right) / \sigma_{i}^{(X)}$ and the normalised growth rate of cost $\left(Y_{i}(t)-\left\langle Y_{i}\right\rangle_{t}\right) / \sigma_{i}^{(Y)}$, respectively. Here $N_{s}$ is the number of suppliers, $\left\langle X_{i}\right\rangle_{t}$ represents the time average of the time series $X_{i}(t)$, and $\sigma_{i}^{(X)}$ represents the standard deviation of the time series $X_{i}(t)$. The set of parameters, $k_{i j}$, denotes the strength of interaction. See also the upper panel of Figure 4.33 for the cost $C_{i}$ of $i$ and the revenue $R_{j}$ of $j$. The normalised growth-rates are related to each other by the above equation.

On the other hand, if we consider the customers in the transaction network, the growth rate of sales for the $i$-th company is represented by

$$
\delta X_{i}(t+1)=\sum_{j=1}^{N_{c}} k_{i j} \delta Y_{j}(t)+\epsilon_{i}(t),
$$

where the first term in the right hand side of this equation corresponds to the interaction by transaction between companies. Here, $N_{c}$ is the number of customers. See also the lower panel of Figure 4.33 for the cost $C_{i}$ of $i$ and the revenue $R_{j}$ of $j$. The normalised growth-rates are related to each other by the above equation.

The parameter $k_{i j}$ is estimated by applying regression analyses with residual error. We performed regression analyses by including $k_{i j}$ for the pairs having statistically significant correlations as explained above. From this analysis we found that the residual error follows a normal distribution with a standard deviation equal to 0.84 , which is smaller than that of the normalised growth rate. This fact means that the introduction of the first term of the right hand side of the equation above was successful.

It is reasonable to expect that we can make residual error still smaller by lowering the confidence level and including more interaction terms. Figure. 4.34 shows the relation between the standard deviation of residual error and the confidence level, which confirms the validity of our expectation.

\section{Network Dependence of the Correlation between Sales}

We will now consider the network dependence of the correlation between companies. To this end, we will consider the shareholding network, the transaction network, and the overlapping network. The overlapping network corresponds to the network in which nodes are connected by both a shareholding relationship and a transaction relationship. In the previous section we stated that the 


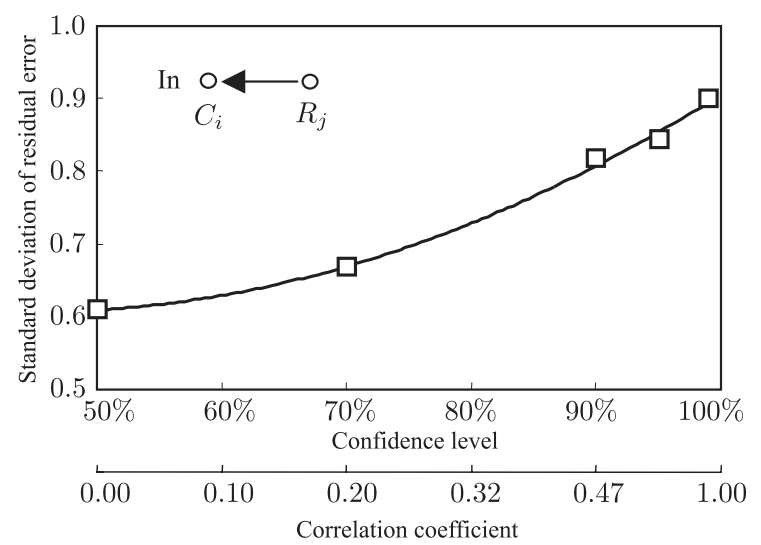

Figure 4.34: Standard deviation of residual error and confidence level

correlation coefficient has meaning for a stationary process, however we will relax this limitation in order to consider the correlation with company sales.

In graph theory the definition of correlation coefficient corresponds to a weighted complete graph in which every node connects to every other node. For example, if we calculate correlation coefficients for 1,400 nonfinancial companies listed in the first section of the Tokyo Stock Exchange by using sales data over the period 2000-2004 (5 years), we can obtain Figure 4.35. The mean value of this distribution is 0.079 . This value is shown in Figure 4.36 as the cross at the length of time series equal to 5 . If we change the length of time series from 4 years (the period 2001-2004) to 20 years (the period 1985-2004), and calculate mean values, we obtain the solid line connecting crosses in Figure 4.36.

If we extract the 1-link correlation (correlation between companies connected by one link) in the overlapping network from the correlation coefficient calculated by using data over the period 2000-2004 (5 years), we obtain the distribution shown in Figure 4.37. By comparing Figure 4.35 and Figure 4.37, we can see that the positive correlation in the overlapping network is larger than the mean value of the correlation coefficient. The mean value of the correlation coefficient in the overlapping network is equal to 0.244 . If we change the length of the time series we obtain the solid line connecting the filled triangles in Figure 4.36. If we apply the same analysis to the part corresponding to the transaction network, we obtain the solid line connecting the filled circles in Figure 4.36. In addition, the solid line connecting the filled squares in Figure 4.36 is for the part corresponding to the shareholding network.

If we extract the 2-link correlation (correlation between companies connected by two links) in the overlapping network and repeat the same calcu- 


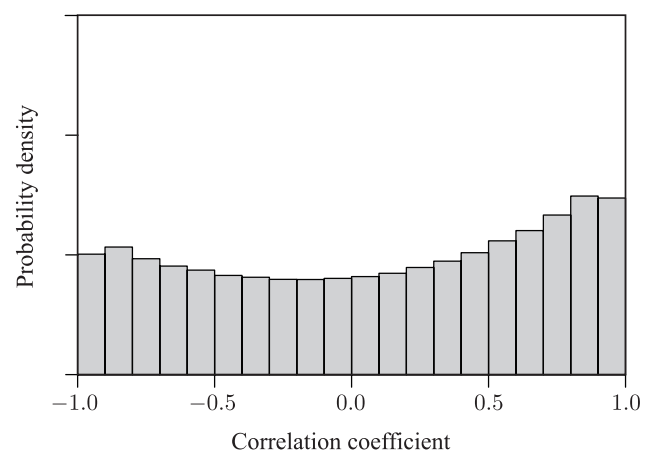

Figure 4.35: Distribution of correlation coefficient for sales

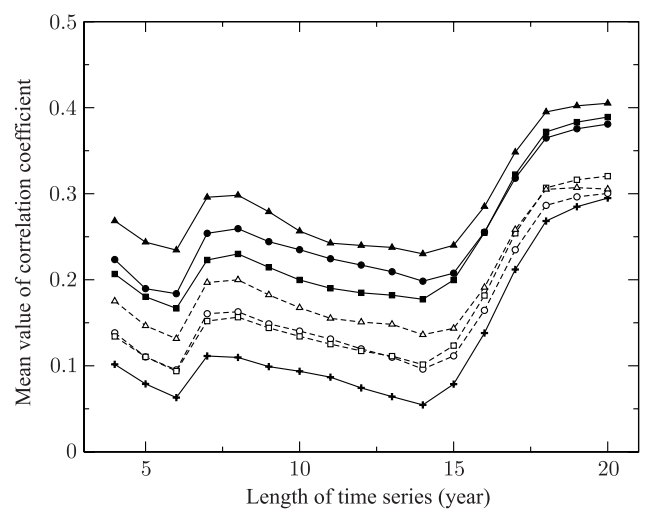

Figure 4.36: Network effect on correlation coefficient 


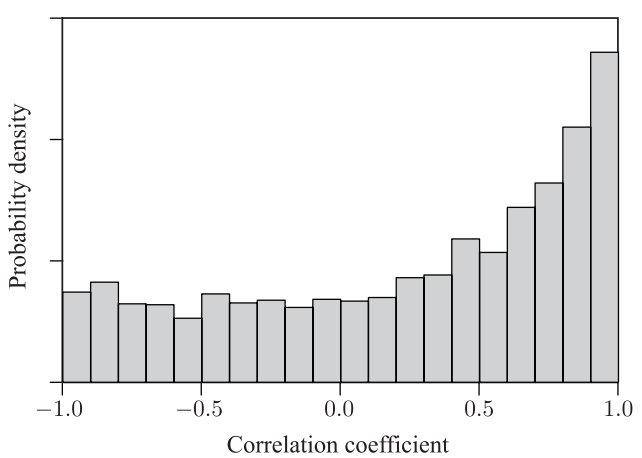

Figure 4.37: Distribution of correlation coefficient for sales in the overlapping network

lation used in the case of the 1-link correlation, we obtain the dashed line connecting the open triangles in Figure 4.36. In this figure, the dashed lines connecting open circles and open squares correspond to 2-link correlations in the transaction network and the shareholding network, respectively. From this figure, we can see, in the case of 1-link correlation, that the correlation in the overlapping network is the largest correlation. This is applicable to the case of 2-link correlation. In addition, the 2-link correlation is smaller than the 1-link correlation for every type of network.

\section{Network Dependence of Correlation between Bankruptcy Probabil-} ities

We can consider the correlation between bankruptcy probabilities. Although many models for bankruptcy probabilities have been proposed we use the bankruptcy probability model known as SAF2002 (Simple Analysis of Failure 2002) (Shirata, 2004). SAF2002 is defined by

$$
\mathrm{SAF} 2002=0.0104 x_{1}+0.0268 x_{2}-0.0661 x_{3}-0.0237 x_{4}+0.7077 .
$$

Here, $x_{1}, x_{2}, x_{3}$, and $x_{4}$ correspond to retained earnings to total assets, net income before tax to total assets, an inventory turnover period, and interest expenses to sales, respectively. This bankruptcy probability was derived by applying nonlinear regression for these four financial items, which differ remarkably between bankrupted companies and non-bankrupted companies.

As is obvious from its name, SAF2002 was proposed in 2002, and consequently it is widely supposed that the model is inapplicable to earlier years. However, we assume that this model is so applicable, and we can calculate the correlation coefficient between bankruptcy probabilities over the period 2000-2004 (a five year period). Thus, we obtain the mean value of correlation coefficient equal to 0.0863 . On the other hand, for a 1-link correlation the 
mean values of correlation are $0.1345,0.1614$, and 0.1780 in the transaction network, the shareholding network, and the overlapping network, respectively. For 2-link correlation, the mean values of correlation are $0.1136,0.1334$, and 0.1677 in the transaction network, the shareholding network, and the overlapping network, respectively.

From these results we can see that the overlapping network has a strong correlation. However, compared with the correlation between sales the network dependence of the correlation between bankruptcy probabilities is weak. In addition, the difference between the 1-link correlation and the 2-link correlation is small. This finding indicates that chain bankruptcy is unlikely. We can consider two underlying causes for this fact. One possibility is that SAF2002 is not applicable to network structure phenomena such as chain of bankruptcy, because SAF2002 indexes only the bankruptcy probability of the company itself. Another possibility is that our data covers only listed companies, for which chain bankruptcy is rare. 


\section{Chapter 5}

\section{Agent-Based Model for Companies}

In Chapters 2 and 3 we investigated various statistical properties of companies by treating them as a statistical ensemble. In the preceding chapter we demonstrated that companies are interconnected through transaction relations, share holdings, cooperative filing of patents and so on, thus resulting in the formation of a complex network. In this chapter we introduce the results of recent studies elucidating the dynamics of interacting companies using agent-based models.

The agents appearing in economic phenomena include consumers, investors, companies, and others, and we can regard economic phenomena as many-body systems in which a number of those agents interact with each other. Such an approach is called an agent-based simulation. Recently more and more simulations of various economic phenomena have been carried out using computers.

An agent in computer science means a small piece of software possessing such properties as autonomy (acting with its own "will"), social nature (collaborating with another) and adaptability (making itself fit to its surroundings). These features of the agent exactly coincide with the agent-based model treated in this book.

So we might say that economic agents are the actors and the stage is a network connecting those agents. But what drama is played out in this theater? We will begin with modeling the dynamics of companies as a stochastic process and then proceed to an explanation of agent-based modeling of companies.

\subsection{Gibrat's Process}

In the present context we refer to the income, assets, and the number of employees attributed to a company as its complete "size". We attempt to 
model the dynamics of companies on the basis of Gibrat's process (Gibrat, 1931), as has been briefly described in Section 3.1.4. The size of a company in a certain year $t$ is denoted by $x(t)$; hence $x(t-1)$ represents the size of the company in the preceding year. Gibrat's law is explicitly expressed as

$$
\frac{x(t)}{x(t-1)}=a(t-1)
$$

where $a(t-1)$ is a stochastic variable with some probability distribution which is statistically independent of $x(t-1)$ and plays a role of growth rate. The law is also rewritten as

$$
x(t)=a(t-1) x(t-1) .
$$

Namely, the size $x(t)$ of a company in this year is $x(t-1)$ multiplied by $a(t-1)$. Such a stochastic process, characterized by multiplication of random variables, is generally called a multiplicative stochastic process. Since Gibrat's process is the simplest among multiplicative stochastic processes, it may be referred to as a pure multiplicative stochastic process.

If we go back one year, Gibrat's law reads

$$
x(t-1)=a(t-2) x(t-2),
$$

and combination of this with the previous expression for $x(t)$ results in

$$
x(t)=a(t-1) x(t-1)=a(t-1) a(t-2) x(t-2) .
$$

Repeating this process, we reach the original or initial time and obtain

$$
x(t)=a(t-1) a(t-2) \cdots a(1) a(0) x(0) .
$$

Let us take a logarithm of both sides of the above equation:

$$
\begin{aligned}
\log x(t)= & \log a(t-1)+\log a(t-2)+\cdots \\
& +\log a(1)+\log a(0)+\log x(0)
\end{aligned}
$$

The right-hand side has been thus converted to additive operations from multiplication. Such a process is called additive stochastic process (Gibrat, 1931) (see also Sutton (1997)).

If various instances of $a(t)$ are uncorrelated to each other, then the central limit theorem states that the distribution of $\log x(t)$ will asymptotically approach the normal distribution as time evolves. Hence the asymptotic distribution of $x(t)$ is given by the log-normal distribution, which is sometimes called Gibrat's distribution. The growth rate $a(t)$ of a company may well depend on the size of the company. However, Gibrat assumed that the size of the company and its growth rate were independent of each other, in other words, that the business chances of a company increased just in proportion to its size. As has been demonstrated by the empirical analyses in Chapter 3, Gibrat's 


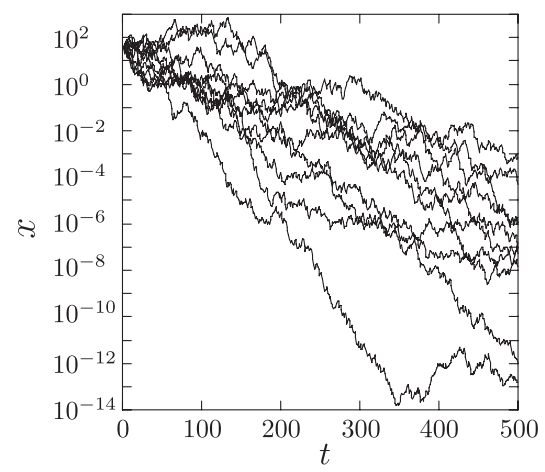

Figure 5.1: behaviour of companies in Gibrat's process

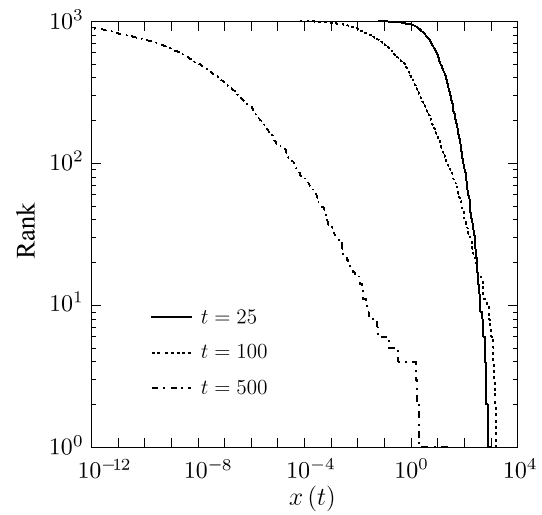

Figure 5.2: Distribution of company sizes in Gibrat's process

law is certainly satisfied by large companies. The log-normal distribution is skewed with a fat tail on the upper side. But the tail is not particularly fat in comparison with that of the power-law distribution. According to this model, diversity in the size of companies continues to be enlarged without any limitation even if they were of the same size at an initial time. However, the ranking of companies by size is not fixed. Figures 5.1 and 5.2 illustrate this interchange of companies. The simulation used one thousand companies and assumed that $a(t)$ took uniform random numbers in $1 / 2<a(t)<3 / 2$. The initial value for $x(t)$ was set as $x(0)=40$. Uniform random numbers were generated by selecting numbers in a given range randomly and with equal probability. 


\subsection{Model of Shareholding Network}

We can now develop the idea of Gibrat's process to construct a model which reproduces the results in Secs. 3.1 and 4.5.4. And using the model we try to shed light on such phenomena as are characterised by the power-law degree distribution. Those phenomena have been demonstrated in Section 4.6.2.

\subsubsection{Reproduction of Size Distribution}

How can we derive a power-law distribution on the basis of Gibrat's process? Physicists have already prepared several answers to this question. One of them is to reset the stochastic variable to its initial value with a certain probability $q$ (Manrubia and Zanette, 1999). This can be expressed as

$$
x_{i}(t)= \begin{cases}a_{i}(t-1) x_{i}(t-1) & \text { with probability } 1-q \\ x_{i}(0) & \text { with probability } q\end{cases}
$$

where $i$ is an index distinguishing companies. Companies do not interact with each other in this model and they have their own evolutionary paths with different statistical uncertainties. The process is referred to as a multiplicative stochastic process with a reset event.

Suppose that the parameter $q$ represents a bankruptcy probability for companies. In this model, as long as companies do not fall into bankruptcy, they evolve according to Gibrat's process. Once a company becomes bankrupt, it restarts its business under the initial condition or is replaced by a newcomer with the same condition. Actually such a simple process is able to explain the outline facts such as the power-law distribution of the sizes of companies, and the exponential distribution of company lifetimes. Alternative models which can reproduce the power-law distribution include multiplicative stochastic processes with a reflection wall (Solomon and Levy, 1996) or with additive noises (Sornette, 1998; Sornette and Cont, 1997; Takayasu et al., 1997). The reflection wall totally reverses the direction of evolution of a company whose size becomes smaller than some lower limit. The multiplicative stochastic process with additive noises was studied in detail by Kesten (1973), and is sometimes referred to as Kesten's process.

We are now in a position to carry out simulations in the reset-event model described above. It is however necessary to specify the simulation parameters. We used 50,000 companies and set $x_{i}(0)=1$ as the initial condition. We also adopted $q=0.005$ for the reset probability. This value arises from the historical fact that Japanese companies went into bankruptcy with annuallyaveraged rates ranging from $0.2 \%$ to $1.7 \%$ during the period of 1970 through 1997. Finally, the growth rate $a_{i}(t)$ was assumed to take random numbers uniformly distributed in $(0.5,1.5]$. Figure 5.3 exemplifies the results obtained by the simulation. This is a log-log plot of the size $x$ of companies on the ordinate versus the corresponding rank on the abscissa for results at a different time. We can readily observe that the distribution stabilises as time evolves. In particular, the distribution is almost stationary beyond $t=200$. 


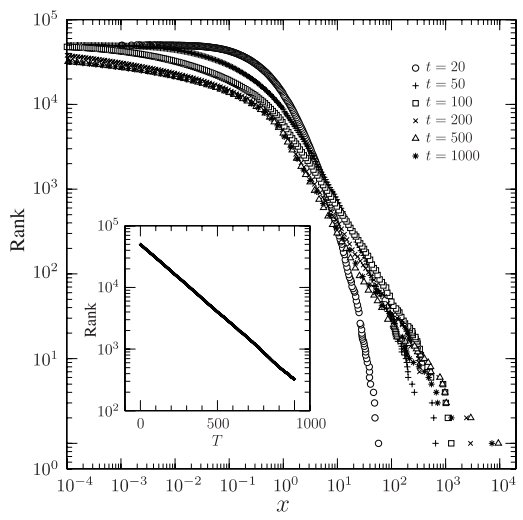

Figure 5.3: Simulated results for sizes and ages of companies

We note that the stable distribution has a power-law tail and its exponent is approximately $\mu=1$. The inset in Figure 5.3 shows a distribution of the ages of companies at $t=1000$. This is a linear-log plot of the age $T$ of companies on the ordinate versus the corresponding rank on the abscissa. The linear behaviour of the distribution demonstrates the exponential characteristics of the distribution. We have thus succeeded in explaining half of the results given in Section 4.5.4.

\section{E. The Weber-Fechner law}

In 1834 E. H. Weber experimentally established that the perceived intensity of physical stimuli is proportional to relative change of the magnitudes of the stimuli, not to their absolute change. Further, in 1859 Fechner mathematically formulated Weber's observation by remarking that our perception intensity is proportional to the logarithm of the stimulus intensity. Everybody experiences saturation of perception in response to increasing physical stimuli. The experimental fact is really a characteristics of the logarithmic function. As well as our five senses, our sense of money, if one may call it that, seems to obey the Weber-Fechner law, according to a recent experiment carried out by one of the authors. The modeling of behaviour of stock prices as a geometric Brownian process may be related to this observation; the rate of return is adopted as a fundamental stochastic variable instead of the stock price itself. Fechner is thus known to be a founder of psychophysics. In fact, he also formulated a statistical concept regarding Kendall's $\tau$, introduced in Chapter 3. 


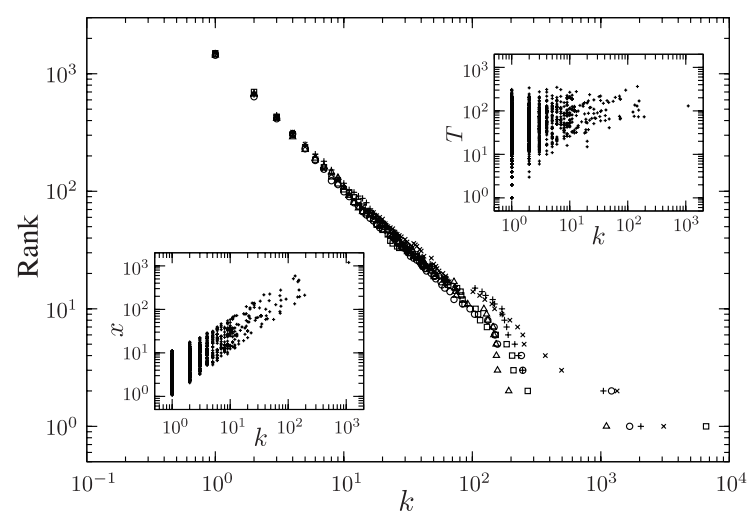

Figure 5.4: Conversion of the simulated results to the degree distribution

\subsubsection{Reproduction of Degree Distribution}

To explain all of the results in Secs. 4.5.4 and 4.6.2 on the basis of the present model we need an additional creative device. To that end we will try to convert the assets possessed by a company to the degree of its links. Companies possess part of their assets in the form of stocks. The percentage of investment in stocks in the sum of total assets differs from company to company, as does the division of the investment funds into different stocks. We will simply define the degree $k_{i}(t)$ of company $i$ as $k_{i}(t) \equiv x_{i}(t) / r_{i}$ where the assets $x_{i}(t)$ are divided by a uniform random number $r_{i} \in[1,5)$.

Figure 5.4 shows the results for the degree distribution obtained by several simulations at $t=1000$. This figure plots the simulated results with the degree $k$ on the abscissa and the corresponding rank on the ordinate, both in a logarithmic scale. We see that the distribution has a power-law behaviour over a wide range. Its power-law exponent is estimated as being close to $\gamma=2$, which is slightly larger than the value in Table 4.5 .

The inset placed at the upper-right corner in Figure 5.4 displays a typical example of correlations between the degree $k$ and the age $T$ of companies at $t=1000$. This figure is a $\log -\log$ plot of $k$ on the abscissa and $T$ on the ordinate. The correlation is weak, and is quantitatively confirmed by the value $\tau=0.21$ for Kendall's $\tau$. This agrees well with the result given in Section 4.5.4. On the other hand, the inset at the lower-left corner in Figure 5.4 exemplifies correlations between the degree $k$ and the assets $x$ of companies at $t=1000$, both of which are plotted in a logarithmic scale. The correlation with $\tau=0.60$ is assessed as rather strong. This is also in good agreement with the result in Section 4.5.4. 


\subsubsection{Effects of Nodal Characteristics}

We have proposed a successful model to explain the power-law behaviour of the degree distribution for companies. At this point we will turn to the remaining issue of why the distribution has an exponentially-decaying tail, a matter raised at the end of Section 4.6.2.

In 1999 Barabási and Albert worked out a simple model to reproduce networks with scale-free characteristics, a model which, for brevity, is often referred to as the BA model (Barabási and Albert, 1999). The key ingredients in their model are network growth and preferential attachment. By network growth we mean that the number of nodes in the network increases over time, and by preferential attachment we mean that nodes with a higher degree have a larger probability of establishing links with new nodes added to the network; in the original model the attachment probability is proportional to the degree of the existing nodes.

In 2000 Amaral and his collaborators demonstrated that the degree distributions of some networks are characterised by an exponential form instead of a power law (Amaral et al., 2000). They then tried to extend the BA model to explain their finding, and in the process they modified the BA model by setting an upper bound to the number of links that a node can have. They interpreted the upper bound as arising from aging or the limited capacity of nodes. That is, the aging effect prevents old nodes from connecting to a new node. Alternatively nodes with a large degree of connections suffer from high cost necessary to keep existing links, so that they have no capacity to make a new connection beyond a critical value $k_{\max }$ for the degree.

In view of the outline facts of the shareholding network, we will assess the validity of the model due to Amaral et al. for studying the tail of the degree distribution. Because the model is a generalised version of the BA model we will initially focus on whether the network is growing with a preferential attachment or not.

Table 4.5 confirms that the network is actually growing although the growth rate is fluctuating over time. Also we note that the tail of the degree distribution mainly stems from contributions by financial institutions, which take positive action in spending their money on investment in new companies. The larger financial institutions are, the higher probability they have of acquiring new stocks. This is really a preferential attachment process working for financial institutions. The key postulates in the BA model thus seem to be satisfied in the shareholding network. So how about effects of the aging and the limited capacity, which are the new ingredients to the BA model introduced by Amaral et al.

In the case of financial institutions, it is hard to imagine that they tend not to acquire new stocks as they become older. And it is difficult to treat their ages in a systematic way because M\&A's occur regularly. On the other hand, the total amount of investment money is fixed for financial institutions so that they have a limited capacity to establish shareholding connections. We thereby claim that the model of Amaral and his colleagues can account for the 
exponential tail in the degree distribution. The next problem is how we can estimate the critical value $k_{\max }$ from the real data. This is reserved for future study. We expect that $k_{\max }$ depends on how much money for investment each financial institution possesses.

\subsection{Balance Sheet Dynamics}

An agent-based model can be used to elucidate complex phenomena encountered in a wide variety of social and economic systems, and is a natural extension of the atomic concept worked out for describing physical systems. Agents have internal structures characterised by different parameters. For instance, agents are made sufficiently intelligent as to be autonomous; that is, an agent has the capability to adapt itself to surrounding conditions. In addition, agents interact with each other according to simple rules. A complex system is thus regarded as an assembly of interacting agents.

D. Delli Gatti and M. Gallegati and their collaborators constructed a promising model consisting of interacting economic agents to describe the dynamics of companies (see Delli Gatti et al. (2000); Gallegati et al. (2003) for the original work and also Delli Gatti et al. (2008)). They demonstrated that the model successfully reproduced a set of stylised facts including the company size distribution with a power-law tail, and the Laplace-type distribution of the growth rate of companies.

A large collection of agents with identical characteristic parameters and a monopolistic bank constitute the model as shown in Figure 5.5. The dynamics of the agents are characterised by their balance sheets. Each company tries to maximise its expected profit against a background of the possible risks in the market. The companies, which are mutually interacting through the bank, become heterogeneous in the course of temporal evolution, and the possibility of bankruptcy is also taken into account. Such a microscopic model, once established, enables us to investigate the interplay between the behaviour of individual companies and the macroscopic trend of economy. We are now in a position to calibrate the model thanks to the accumulation of results relating to the statistical properties of the dynamics of real companies.

We will initially reconstruct the original model to elucidate the conceptual ingredients. The compromise between the two concepts, profit maximisation and maintaining the company as a going concern plays a key role in the decision making of companies using imperfect information relating to their financial conditions. Simulations based on the model are then carried out for the statistical properties of company dynamics and the results so obtained are discussed in light of observations in the real economy. For more details of the contents given in this section, we refer the readers to Iyetomi et al. (2009a). 


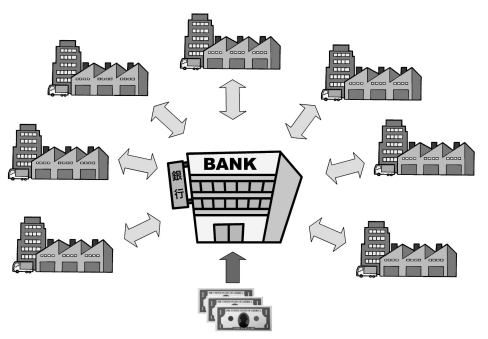

Figure 5.5: Conceptual figure of agent-based model: companies interacting through a single bank

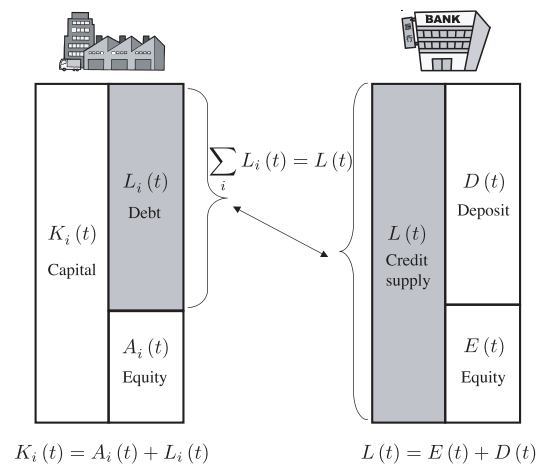

Figure 5.6: balance sheets for companies and a bank

\subsubsection{The Basic Agent Model}

The dynamics of the agents are described in terms of balance sheets as shown in Figure 5.6. A company $i$ has total capital $K_{i}(t)$ and debt $L_{i}(t)$ from the bank at the beginning of a time period $t$. Here, $i=1, \ldots, N$ and $N$ is the total number of companies. According to the accounting equation, the equity capital $A_{i}(t)$ of the company must equal the total capital minus the debt. On the other hand, the bank agent has a balance sheet on which its aggregate supply of credit, $L(t)=\sum_{i} L_{i}(t)$, is balanced by the sum of total deposits $D(t)$ and equity capital $E(t)$. Stocks and flows are two kinds of basic variables to construct models of system dynamics. balance sheets have only stock variables so that they are just snapshots in time of a company's financial condition. Flow variables such as profit and investment determine evolution of the economic system.

For the sake of simplicity when we model a company's dynamics we con- 


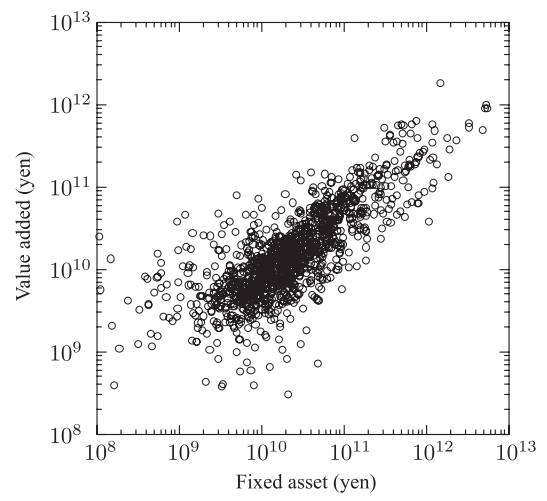

Figure 5.7: Value added versus fixed assets for listed Japanese companies in 2003

centrate on the financial aspect of the production function and assume the value added $Y_{i}(t)$ linearly scales to the capital input:

$$
Y_{i}(t)=\phi K_{i}(t)
$$

where the proportionate $\phi$ is taken as $\phi=0.1$. Figure 5.7 validates this modeling for the production function.

At the beginning of a given time period $t$ the $i$-th company changes its capital $K_{i}(t)$ to maximise the expected value of profit. This strategic behaviour of the company, called profit maximisation, is a well-known hypothesis in economics since the time of Adam Smith, although it has not yet been confirmed.

The profit of a company is fixed at the end of each period as

$$
\pi_{i}(t)=u_{i}(t) Y_{i}(t)-r_{i}(t) K_{i}(t)=\left[u_{i}(t) \phi-r_{i}(t)\right] K_{i}(t),
$$

where $r_{i}(t)$ is the interest rate for financial cost. The parameter $u_{i}(t)$ reflects uncertainty in the market. Since a market consists of a huge number of economic degrees of freedom, the determination of the selling price becomes inevitably stochastic. We also assume that $u_{i}(t)$ is independent of the company size in harmony with Gibrat's law. We arbitrarily take $u_{i}(t)$ as a uniform random number in $(0,2)$. Expected profit is thus given by setting $u_{i}(t)=1$ in the equation for $\pi_{i}(t)$ as

$$
\left\langle\pi_{i}(t)\right\rangle=\left[\phi-r_{i}(t)\right] K_{i}(t)
$$

At the lower limit $u_{i}(t)=0$, products are so discounted that they are unable to yield value added, and maximum value added is obtained at a price double the expected price corresponding to $u_{i}(t)=2$. 

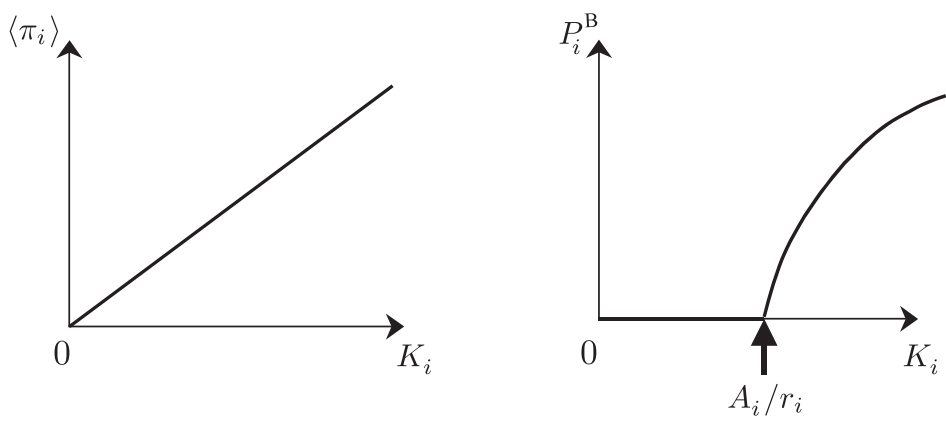

Figure 5.8: Emergence of finite probability of bankruptcy with increase of expected profit

If a company adopts an aggressive production plan it has a finite probability of bankruptcy. The bankruptcy of a company is defined at the end of a period $t$ by the condition,

$$
A_{i}(t)=A_{i}(t-1)+\pi_{i}(t-1)<0 .
$$

Substitution of the previous equation for $\pi_{i}(t-1)$ into the bankruptcy criterion results in the following formula for the bankruptcy probability:

$$
P^{\mathrm{B}}\left(K_{i}(t)\right)= \begin{cases}\frac{r_{i}(t) K_{i}(t)-A_{i}(t)}{2 \phi K_{i}(t)} & \text { for } K_{i}(t)>\frac{A_{i}(t)}{r_{i}(t)}, \\ 0 & \text { otherwise. }\end{cases}
$$

We thus see there is an upper bound in the size needed for a company to be free from bankruptcy. The relationship between the increase of expected profit and the emergence of bankruptcy is depicted in Figure 5.8.

Another management policy, known under the label of the going concern policy, prevents a company from expanding its size infinitely; a company aims to survive forever. We assume here that companies adopt a solid production plan with a safety factor $\sigma(\leq 1)$ :

$$
K_{i}(t)=\sigma \frac{A_{i}(t)}{r_{i}(t)}
$$

This choice compromises two directly-opposed economic ideas.

The interest rate for each company is then determined through the demand and supply balance in the credit market inhabited by companies and the bank. The company requests the bank to finance the following amount of money derived from the equation for $K_{i}(t)$ :

$$
L_{i}^{d}(t)=K_{i}(t)-A_{i}(t)=\left(\frac{\sigma}{r_{i}(t)}-1\right) A_{i}(t) .
$$




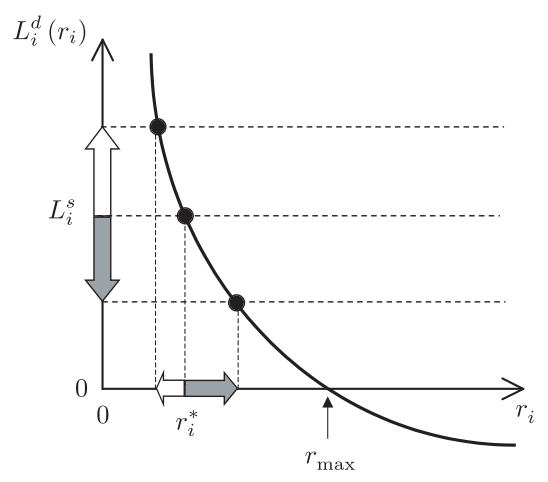

Figure 5.9: Determination of the interest rate for a company

On the other hand, credit is granted to the company by the bank in proportion to its relative size in the preceding period as

$$
L_{i}^{s}(t)=L(t) \frac{K_{i}(t-1)}{\sum_{i} K_{i}(t-1)},
$$

Balancing $L_{i}^{d}(t)$ and $L_{i}^{s}(t)$ gives the formula for the interest rate,

$$
r_{i}(t)=\frac{\sigma A_{i}(t)}{L_{i}^{s}(t)+A_{i}(t)} .
$$

Such an equilibrium mechanism to determine the interest rate is depicted in Figure 5.9. The maximum rate is given by $r_{\max }=\sigma$. If the company obtains more credit from the bank, the interest rate decreases, and vice versa.

Companies with the above-mentioned behavioural rules would never go bankrupt, but real companies, of course, are always afraid of bankruptcy. To incorporate the possibility of bankruptcy into the model, we will replace the equity capital of the current period by that of the preceding period in the previous equation for $K_{i}(t)$ :

$$
K_{i}(t)=\sigma \frac{A_{i}(t-1)}{r_{i}(t)} .
$$

Companies thus determine their production plans with delayed information. This replacement overturns the conservative attitude of companies when they are in a recession phase, and the companies incidentally take speculative management actions. We can arbitrarily set $\sigma=1 / 2$, which enables us to make a smooth connection with the original model. The present equations are seamlessly reduced to the corresponding equations in Delli Gatti et al. (2008) by omitting the intensive terms which are independent of the dimensions of the agents. 
Delay of information is one of causes of bankruptcy for companies. Alternatives include the existence of unexpected risk, and the propagation of bankruptcy akin to a chain reaction. In our analysis so far the possible risk is supposed to be totally predictable by specifying a definite range for the stochastic parameter $u_{i}(t)$, but nobody can avoid unexpected risk in real business. In fact, companies are linked to each other through transactions and with a supply of credit. If a large company is bankrupted, then a credit risk shock will propagate over the network. The chain reaction bankruptcy arising from such direct interactions among companies is beyond the scope of the present section and will be discussed later in this book.

With regard to the dynamics of the bank we assume that the bank expands its business subject to the minimum requirement of a prudential rule with a risk coefficient $\alpha$ :

$$
L(t)=\sum_{i} L_{i}(t)=\frac{E(t)}{\alpha} .
$$

The Basel committee of the Bank for International Settlements introduced an international capital adequacy standard called Basel I in $1988 .{ }^{1}$ It requires that each bank has capital equivalent to at least $8 \%$ of the total asset: $\alpha=$ 0.08. The bank derives a profit through investing its money in companies. Here the profit margin is set as $0.2 \%$. However its net profit $\Pi_{t}$ is given by subtracting financial costs from the sum of interests. The financial costs are interest payments paid to depositors and investors along with additional loss due to bad debts stemming from bankruptcy of companies.

\subsubsection{Representative Agents}

Before discussing the results for multi-agent simulations, we will first study a simple system comprising a single ideal company interacting with the bank agent; companies are thus represented by a single agent. This representative agent model, neglecting the heterogeneity of agents, is a traditional approach in economics. Figure 5.10 shows that both agents grow exponentially. This is an intrinsic property of the present model. For a market with no fluctuations in the selling price $\left(u_{i}(t)=1\right)$ and in the interest rate $\left(r_{i}(t)=r\right)$, one can obtain an analytic solution with exponential growth for each agent. Equating two formulae for the growth rate of the representative agents, one can determine the average interest rate $r$ and hence the growth rate. The growth rate derived analytically in this way provides a very good explanation of the results in Figure 5.10.

\subsubsection{Reduction to a Multiplicative Process}

One can terminate the interaction by assuming

$$
r_{i}(t)=\phi
$$

${ }^{1}$ Basel I is to be replaced by the Basel II with more refined rules. 

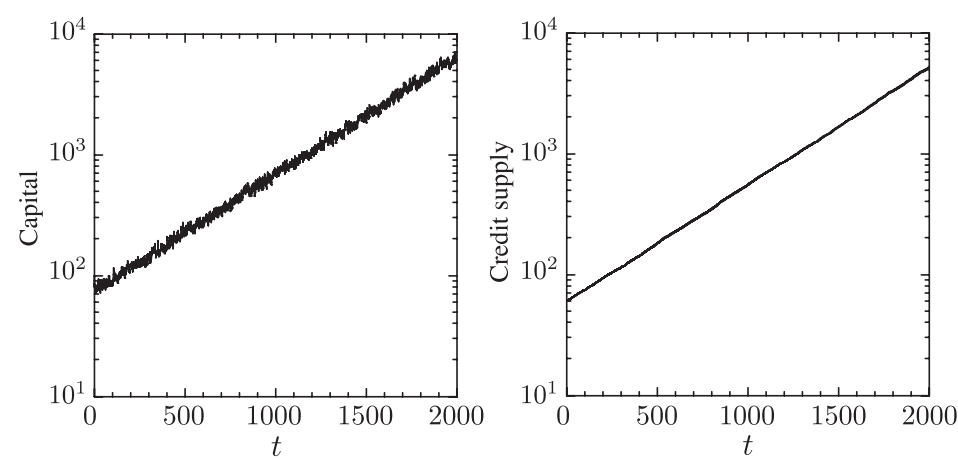

Figure 5.10: Representative company (left panel) and bank (right panel)

The profit for companies vanishes on average in such a situation, corresponding to a zero-growth economy without competition between companies. This assumption reduces the present model with ideal companies to a random growth model:

$$
A_{i}(t+1)=\lambda_{i}(t) A_{i}(t)=\frac{1}{2}\left[1+u_{i}(t)\right] A_{i}(t)
$$

with

$$
\left\langle\lambda_{i}(t)\right\rangle=1 .
$$

We thus see that Gibrat's process underlies the present model. If companies do not go into bankruptcy their size distribution approaches the log-normal form, but is ever-growing. The system constituted by companies with finite probability of bankruptcy reaches a stationary state in which the size distribution is represented by the Pareto distribution with $\mu=1$.

\subsubsection{Distribution of Company Sizes}

We executed numerical simulations based on the present model with 100,000 companies. This number of companies is still much smaller than the actual number of existing companies in Japan, at about 2.5 million. We started the simulations by giving all companies identical initial conditions. When, as time passed, companies went into bankruptcy, they were replaced by new ones with the same initial conditions. That is, the total number of companies was kept constant during the simulations. Although companies were completely equal at the outset, they became differentiated and some of them died and were replaced by new companies. Eventually companies were divided into classes of large, medium, and small sizes. This evolutionary process of companies is depicted in Figure 5.11. 


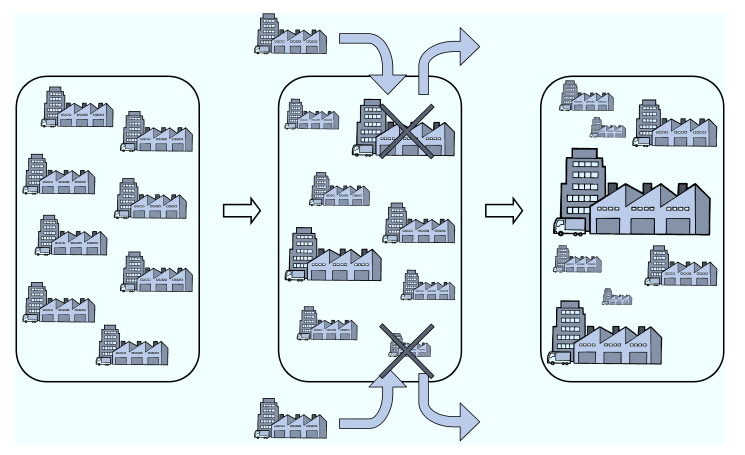

Figure 5.11: Temporal evolution of the agent-based simulation

Figure 5.12 exemplifies the temporal behaviour of companies in the present model, and are results obtained with small numbers of companies $(N=$ $2,3,1000)$. Note that the tracks of companies are very irregular and that there is competition amongst companies. This is in sharp contrast to the representative agent model in which a single company steadily grows together with the bank. We thus see that heterogeneity of companies is a natural outcome of the competition amongst companies interacting through the bank.

Figure 5.13 shows how the bank evolves in time. This is a result for a bank dealing with a group of actual companies with possibilities of bankruptcy. Its equity fluctuates appreciably and sometimes encounters large shocks, such idiosyncratic shocks stemming from the bankruptcy of large companies. On the other hand, the growth of the bank is very steady for ideal companies without bankruptcy; its rate is almost indistinguishable from that of the representative company.

In Figure 5.14, we plot the size distribution of ideal companies without bankruptcy. The left panel shows the result obtained when the bank evolves naturally with exponential growth, while the right panel is the result when the size of the bank is artificially fixed, that is a stationary economy is realized. Both distributions are well fitted to the log-normal form, which is in keeping with Gibrat's process in its simple form. In Figure 5.15, we display the size distributions for actual companies susceptible to bankruptcy corresponding to the two economic conditions in Figure 5.14. The sizes of those companies are distributed in a power-law form:

$$
\operatorname{Rank} \propto K_{i}(t)^{-\mu} .
$$

When the bank grows naturally the Pareto exponent is tentatively stabilised around $\mu=2$ and then approaches $\mu=1$ as time proceeds. We thus see that the size distribution of companies and the temporal evolution of the bank critically depends on whether companies make full use of available information 


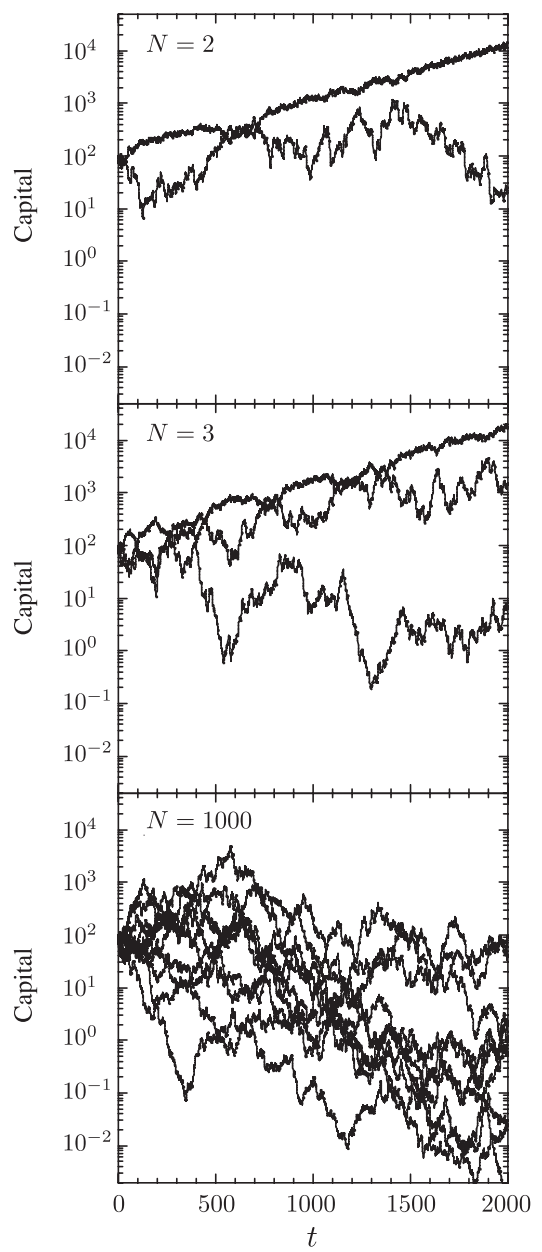

Figure 5.12: Competition among companies 


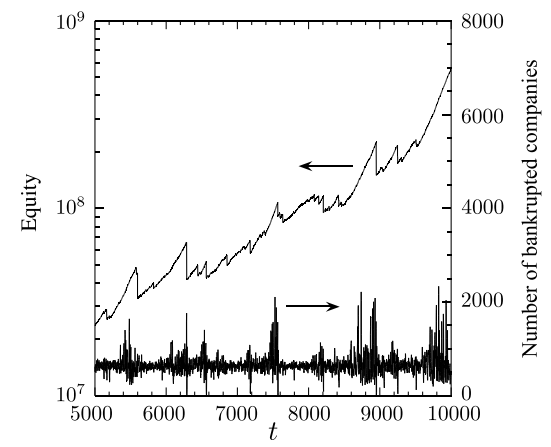

Figure 5.13: Temporal evolution of the bank agent
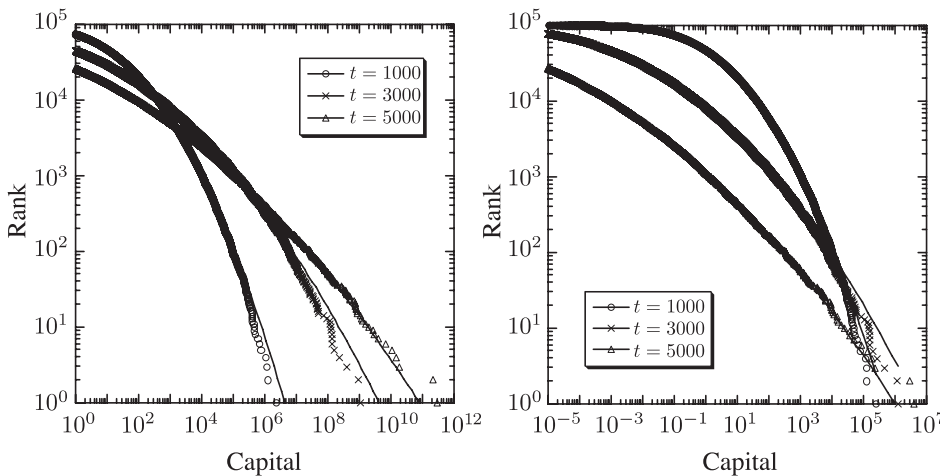

Figure 5.14: Size distribution for companies existing eternally

on their financial conditions or not in determining a production plan for the next period. If we control the macroscopic economy by keeping the size of the bank fixed, we have a stationary state with a power-law size distribution as shown in the right panel of Figure 5.15. The exponent is close to $\mu=2$. This value is totally different from the exponent $(\mu=1)$ found for non-interacting companies. The interactions among companies through the bank give rise to profound changes in the statistical properties of companies as manifested by the variation of the Pareto exponent.

\subsubsection{Synchronised Bankruptcy}

Bankruptcies of companies take place in a synchronised way with macroscopic shocks reflected in the equity capital of the bank. Figure 5.16 enlarges Fig- 

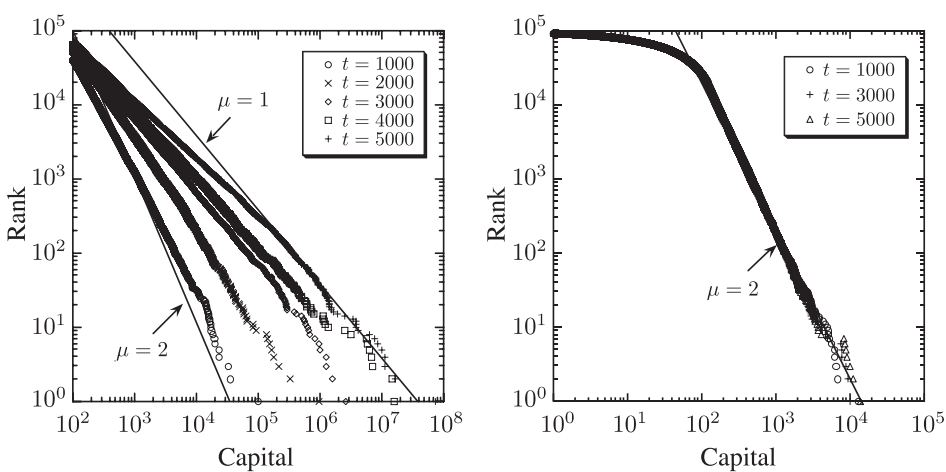

Figure 5.15: Size distribution for companies susceptible to bankruptcy

ure 5.13 to confirm the emergence of such synchronised bankruptcy. The bankruptcy of a large company triggers a chain of bankruptcies in the present model, which takes into account interactions amongst companies. The large bankruptcy gives rise to large bad debts for the bank. Then the equity capital of the bank shrinks and accordingly the bank's credit supply to other companies decreases. This leads to an increase of interest rates for loans from the bank and hence decrease of profits for companies. Financially fragile companies with a low equity ratio $A_{i}(t) / K_{i}(t)$ are thus strongly affected by the bankruptcy of the large company.

\section{Toward an unequal society}

Socioeconomic disparity is growing throughout the world, and is emerging as a serious social problem which should be solved. Equality in every aspect is not a solution, however. As has been demonstrated by the agent-based simulations introduced here, heterogeneity of agents in an economic system is a natural outcome of competition amongst agents with both growth and failure, giving rise to vitality in the system. Bearing in mind Pareto's law we expect that many readers may be on the "useless" side of the distribution, but they may be exactly the people to lead society in the near future. Everyone should prepare themselves for taking over the pole position on a routine basis. What must be avoided is a society in which there is no potential for challenge and the current disparity is thereby fixed. A sound society is fluid and continuously promotes the interchange of positions. Having only a single measure for evaluation is one of major causes of fixing disparities in society. For example, the concentration of research resources is recently observable in the academic world in Japan. This tendency may eventually lead to loss of diversity in research activities. We thus have reason to be concerned that the pursuit of tentative and perhaps illusory efficiency may 

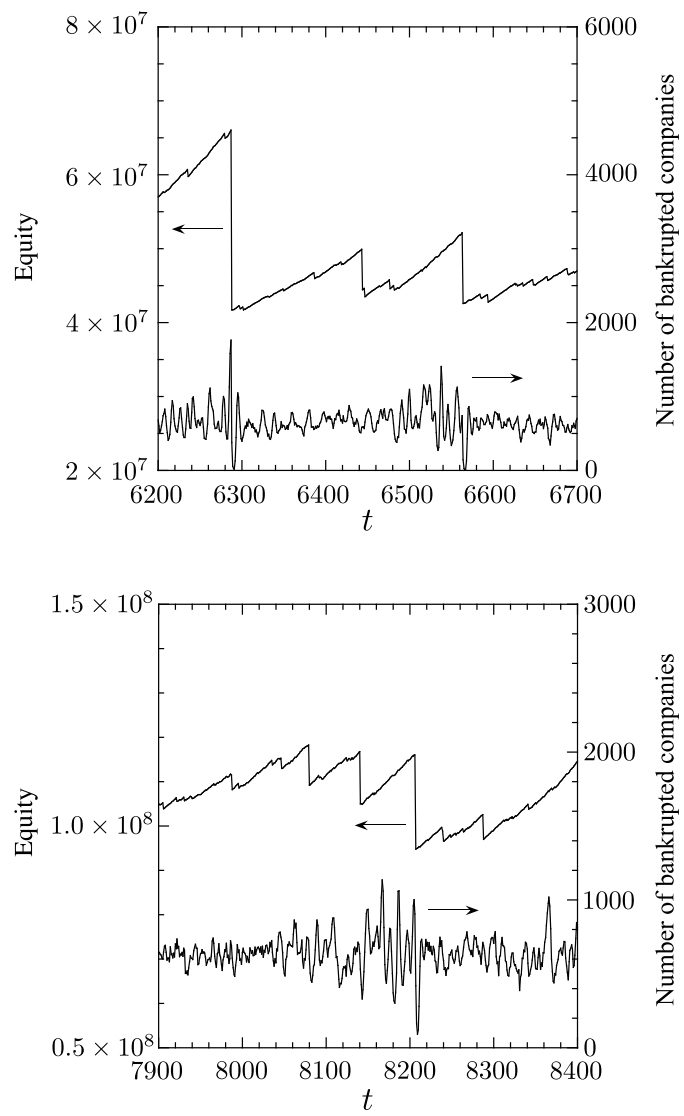

Figure 5.16: Macroscopic shocks originating from synchronised bankruptcy 


\subsection{Network Effects on Wealth Distribution}

Recent development in the means of transport and communication enables companies to connect with each other in spite of physical distances. It is indispensable to take account of direct interactions between companies arising from such an economic network to elucidate various economic phenomena. For instance, we aim to shed light on the origin of business cycles, the stability of economic systems against external shocks or large bankruptcies, and the formation of industrial clusters.

In the previous section, we explained the distributions of company sizes and degrees without any account of interactions between companies. In the present section we will consider a prototype model for such economic interactions. Although the model may be too simple to be applied to actual economic phenomena, we expect that it will be useful for illuminating the effects of the network on the whole economy. The model described here is not limited to interactions between companies, but is generally applicable to any interactions of agents with entities which can be regarded as constituting wealth (Bouchaud and Mezard, 2000; Souma et al., 2001). A typical example of wealth is the total asset base of a company. Here $x_{i}(t)$ denotes the wealth of the $i$-th agent at time $t$.

\subsubsection{Model Construction}

Suppose that the $i$-th and $j$-th agents are dealing with each other for wealth and their dealing is described by $J_{i j}(t)(\geq 0)$. The interaction coefficients $J_{i j}(t)$ constitute a dealing matrix. The present model is then given by

$$
x_{i}(t+1)=a_{i}(t) x_{i}(t)-\sum_{j} J_{j i}(t) x_{i}(t)+\sum_{j} J_{i j}(t) x_{j}(t) .
$$

This is a generalisation of Gibrat's process, with the second and third terms in the right-hand side (Bouchaud and Mezard, 2000). The second term represents the transfer of wealth from the $i$-th agent to the $j$-th agent, so that it takes a minus sign. On the other hand, the third term with a plus sign represents the reverse process. Relative magnitudes of these two terms determine the direction of net flow of wealth. Thus the terms additional to Gibrat's process mimic wealth transactions between agents.

We will now simplify the model so that the dealing matrix $J_{i j}(t)$ is independent of time and given as

$$
J_{i j}=\frac{J}{k_{j}} A_{i j}
$$

where $A_{i j}$ is an adjacency matrix which is constituted by 0 and 1 . If the $i$-th and $j$-th agents are connected (adjacent) to each other through the transaction 


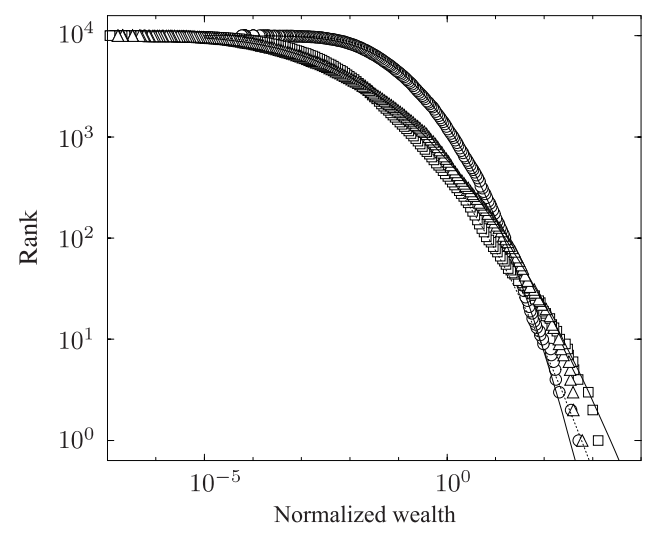

Figure 5.17: Wealth distribution in a regular network

network, $A_{i j}$ takes 1 and otherwise 0 , and $k_{j}$ refers to the degree of the $j$-th agent. In addition, $J$ is a constant and arbitrarily specified. Compilation of the aforementioned simplifications further reduces the model to

$$
x_{i}(t+1)=a_{i}(t) x_{i}(t)-J x_{i}(t)+J \sum_{j \in \operatorname{adj}(i)} \frac{x_{j}(t)}{k_{j}},
$$

where $\operatorname{adj}(i)$ denotes a set of nodes adjacent to the node $i$ in the same way as that on p. 136 .

\subsubsection{Network Effects}

For our simulation of wealth distribution on a given network let us assume that the total number of agents is 10,000 and $J=0.01$. Furthermore, the stochastic variable $a_{i}(t)$ is assumed to obey the normal distribution with an average of 1.01 and variance of 0.02 . Figure 5.17 shows the results so simulated for a regular network of the type depicted by the leftmost panel of Figure 4.1, where the average degree of the network was set to 10 . This figure plots magnitudes of wealth reduced with its average on the abscissa and the corresponding ranks on the ordinate; both axes are on a logarithmic scale. Open circles, squares, and triangles in the figure show the results at $t=4000,12000$, and 20000, respectively. The curves attached to each of the results show the outcome of fitting by the log-normal distribution. We see that the wealth distribution is not stationary and is well described by the log-normal distribution with time-varying average and variance. Also we note that wealth is distributed very unequally over the scale of $10^{7}$.

We will next modify the network structure by keeping the average degree fixed. For instance, the regular network is randomised by rewiring links with 


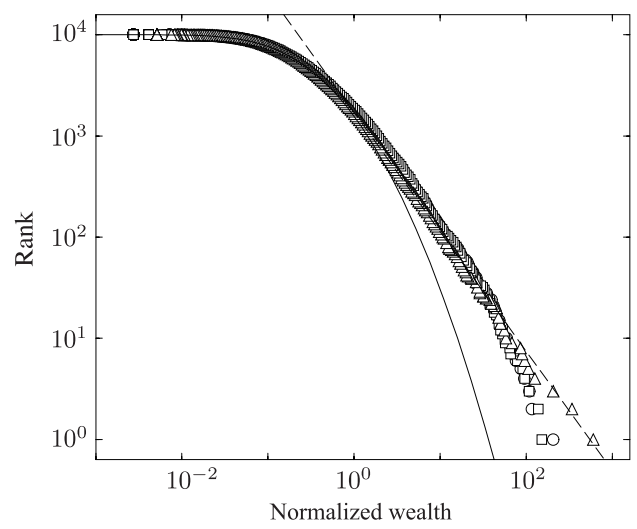

Figure 5.18: Wealth distribution on a small-world network

the probability of 0.05 as depicted in Figure 4.1. The results in such a network are shown in Figure 5.18. The symbols in the figure share the same meanings as those in Figure 5.17, and the dashed line shows the fitting of the tail part of the distribution with a power-law form, and the solid curve shows fitting of the remaining part of the distribution by a log-normal form. As we clearly see from the figure, the small-world network realises a stationary distribution, which is explained by combining the power-law and the log-normal distributions. In the small-world network wealth is distributed over the scale of $10^{5}$. Addition of even a small amount of randomisation processes to the regular network thus results in significant improvement over the uneven distribution observed in the network.

So what happens to a completely random network? Figure 5.19 shows the results. The notations in the figure are exactly same as those in Figure 5.18. The distribution is stationary with a power-law tail and its relative spread is almost confined within the order of $10^{3}$. We see that wealth is distributed much more equally in the random network as compared with the case in a regular one.

\subsubsection{Clustering of Wealth}

In order to elucidate the origin of different distributions of wealth we will focus our attention on how wealth changes its distribution across agents in a given network as time passes. Figure 5.20 displays the temporal evolution of the distribution of wealth in the regular network used in Figure 5.17. The abscissa of the figure is the agent number, ranging from 1 to 1,000, and the ordinate is the time period, $t=0 \sim 10^{4}$. For an agent whose rank as regards magnitude of wealth falls within the top (bottom) $10 \%$, it is depicted by a black (gray) point. We observe those black and gray points make clusters in 


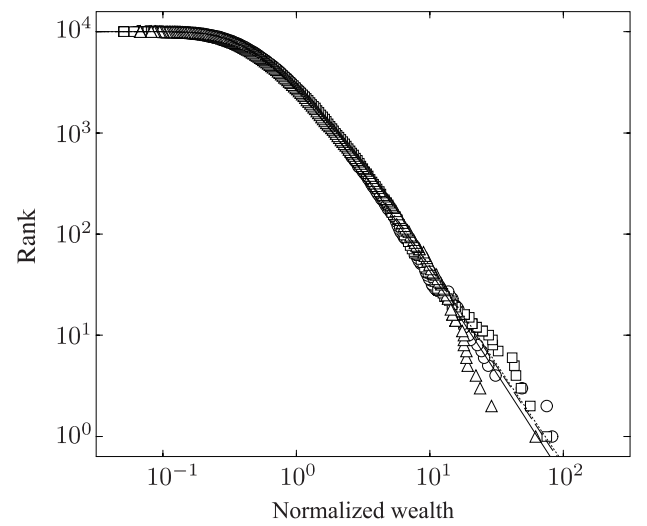

Figure 5.19: Wealth distribution in a random network

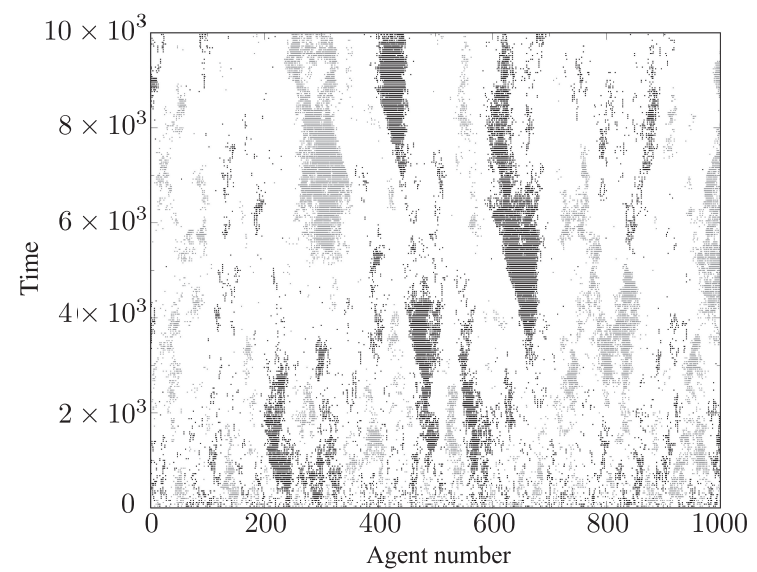

Figure 5.20: Time evolution of distribution of wealth across agents on the regular network, corresponding to Figure 5.17.

the figure. Since neighboring agents are connected to each other in the regular network, this means that linked agents have almost the same magnitude of wealth; namely, clustering of wealth takes place in the regular network.

What happens to the clustering of wealth if we modify the network structure? Figure 5.21 shows the results in the same small-world network as used in Figure 5.18. Note that the clustering tendency of wealth is significantly depressed. The results for the same random network as used in Figure 5.19 


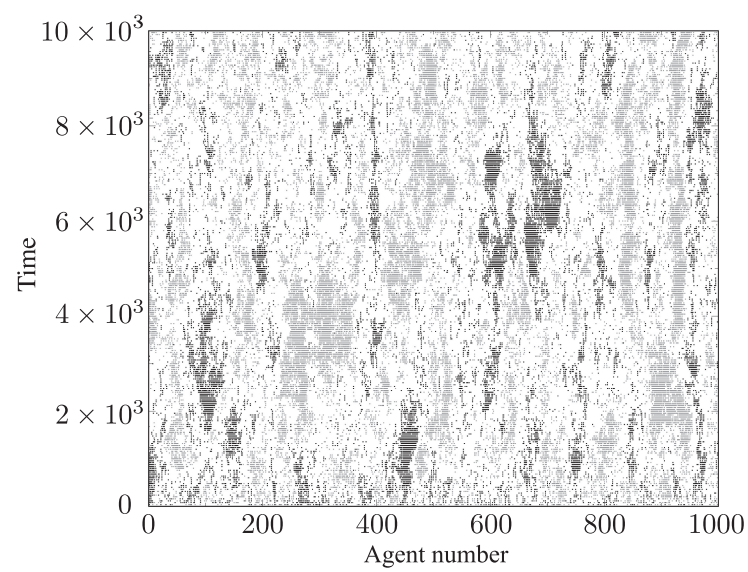

Figure 5.21: Time evolution of distribution of wealth across agents on the small-world network corresponding to Figure 5.18.

are given in Figure 5.21, where no appreciable clustering tendency of wealth is observed. ${ }^{2}$

We have thus learned that the unequal distribution of wealth stems from clustering of wealth in the case of a regular network. The model given on p. 189 has an identical value for all $k_{j}$ in the third term on the right-hand side of the equation, when applied to a regular network, and the number of $\operatorname{adj}(i)$ is identical to $k_{j}$. In addition $x_{i}(t) \simeq x_{j}(t)$ obtains for neighboring agents owing to the clustering of wealth. Accordingly the second and third terms are almost cancelled out. This cancellation mechanism leads to

$$
x_{i}(t+1) \simeq a_{i}(t) x_{i}(t) .
$$

which is simply Gibrat's process.

On the other hand, the rewiring of links in a network gives rise to a difference between the second and third terms on the right-hand side of the model equation. The model given on p. 189 is written as

$$
x_{i}(t+1)=\left[a_{i}(t)-J\right] x_{i}(t)+J \sum_{j \in \operatorname{adj}(i)} \frac{x_{j}(t)}{k_{j}} .
$$

Here the first term on the right-hand side amounts to the original with the average of $a_{i}(t)$ merely shifted by $J$. If there is no correlation between $x_{i}(t)$

\footnotetext{
${ }^{2}$ More strictly speaking, we investigated the correlation between the wealth of the $i$-th agent, $x_{i}(t)$, and the mean wealth of agents $\operatorname{adj}(i), \bar{x}_{i}(t)$, i.e., $\left\langle x_{i}(t) \bar{x}_{i}(t)\right\rangle$ in Souma et al. (2001). Such a numerical analysis confirms our present conclusions on the clustering of wealth over the networks.
} 


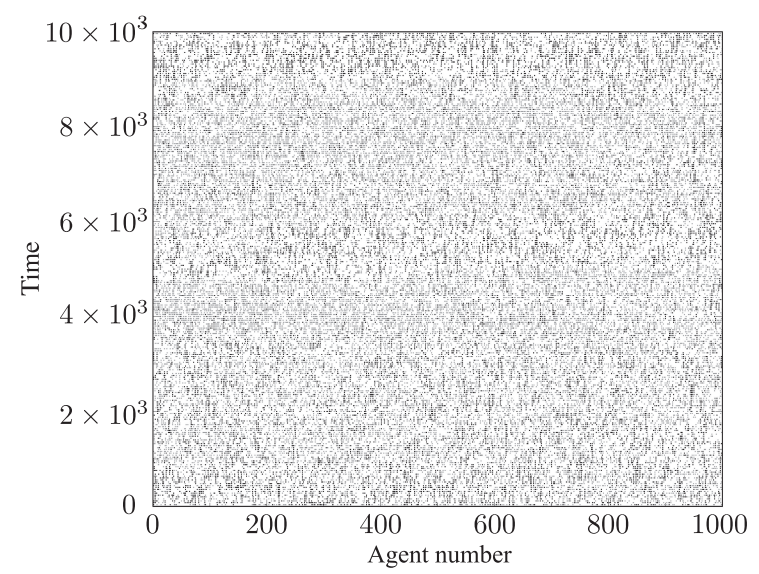

Figure 5.22: Time evolution of distribution of wealth across agents on the random network, corresponding to Figure 5.19.

and the second term on the right-hand side, then this model is reduced to a multiplicative stochastic process with additive noise, and it is well-known that the stochastic process is able to reproduce a power-law distribution. We note that the present model is also applicable to any networks other than the small-world network model of Watts and Strogatz. For instance, we applied the model to the scale-free network derived from BA model and obtained nearly the same results as those in the random network (Souma et al., 2003).

We have introduced a primitive model for interacting agents in this section. However addressing the real economy requires us to continue to develop the modeling. Section 4.6.3 empirically revealed various aspects of interactions between companies. We thereby proceed one step further in the next section to model companies as agents and carry out more realistic simulations.

\subsection{Modelling the Transaction Network}

The most fundamental activity of companies is production. Companies build productive facilities and hire employees. Then companies buy materials to make goods and sell them to generate profits. Companies spend the obtained profits to expand their productive facilities and increase their employees. This is a positive feedback mechanism for the growth of companies. Such production activity apparently requires an individual company to establish connections to other companies for buying materials and selling goods, eventually leading to formation of a transaction network.

In this section we will consider a model for working interactions between 
companies over a transaction network (Ikeda et al., 2007a,b). We aim to develop the arguments given in Section 5.4. In this model companies make autonomous decisions as regards investment in capital and labour to make goods, and they buy the necessary materials and sell goods through transaction relations. We refer to this model as a transaction network model. We will explain the basic ideas of the model one by one below.

\subsubsection{Autonomous Companies}

As has been demonstrated in Section 4.6.3, sales grow according to the following formula:

$$
\delta X_{i}(t+1)=\sum_{j=1}^{N} k_{i j} \delta Y_{j}(t)+\epsilon_{i}(t),
$$

where $\delta X_{i}(t)=\left(X_{i}(t)-\left\langle X_{i}\right\rangle_{t}\right) / \sigma_{i}^{(X)}$ is the standardised sales growth rate. In the definition of $\delta X_{i}(t)$, we employed the time average growth rate $\left\langle X_{i}\right\rangle_{t}$. This basically assumes that economy grows in a constant manner. In fact, however, this assumption cannot be valid, because the actual sales of companies depend heavily on the time-varying business trends of competitors and customers and also the consumption trends of individuals. To take account of this realistic situation, we replace $\left\langle X_{i}\right\rangle_{t}$ by

$$
\left\langle X_{i}\right\rangle_{t} \rightarrow R_{i}^{(G)}(t+1) / R_{i}^{(G)}(t),
$$

where $R_{i}^{(G)}(t+1) / R_{i}^{(G)}(t)$ represents the trend change of the sales growth rate due to the decision making of companies as regards capital $K_{i}(t)$ and labour $L_{i}(t)$. The superscript $(G)$ of $R_{i}^{(G)}(t)$ stands for game theory. The capital $K_{i}(t)$ is the total amount of expenses for facilities necessary to produce goods, and the labour $L_{i}(t)$ is the total amount of wages for employees who operate the production facilities. Companies are able to determine $K_{i}(t)$ and $L_{i}(t)$ autonomously. On the other hand, the sales $R_{i}(t)$ and the costs $C_{i}(t)$ are determined by the transaction relationships between buyers and sellers. A company with such properties is depicted by Figure 5.23. A number of companies thus form a business network through transactions. In passing we note that $\sigma_{i}^{(X)}$ takes a small value if the trend of the sales growth rate changes.

Each company makes decisions about $K_{i}(t)$ and $L_{i}(t)$ to maximise its own profit, and accordingly determines $R_{i}^{(G)}(t)$. So how do companies make their own choices? To grasp their autonomy we can invoke game theory. It should be remembered that companies make decisions about $K_{i}(t)$ and $L_{i}(t)$ using game theory in the present model.

Game theory can be traced back to the seminal work of John von Neumann and Oskar Morgenstern in 1944 and is a mathematical theory to describe the decision making of agents in competition with others (von Neumann and Morgenstern, 1944). Since game theory is full of specific terminologies, many readers may have difficulty understanding it. We will briefly review the terminology often used in the theory and then explain its basic parts. Readers 


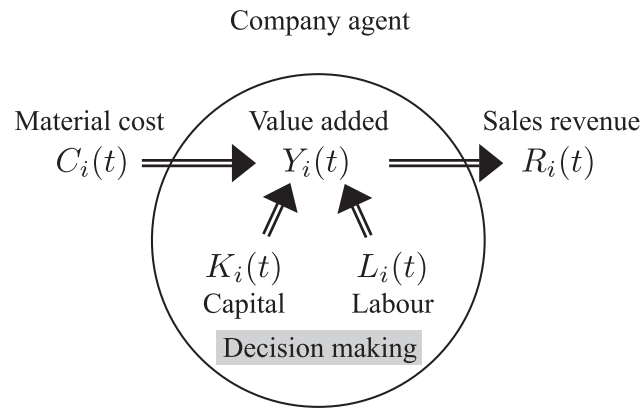

Figure 5.23: Interacting company agents

who are interested in more details of the theory can consult the standard texts (Davis, 1983; Gibbons, 1992; Owen, 1995).

- Non-cooperative game: This is a game in which agents make their own decisions without any cooperation, leading to a competitive situation. The transaction network model assumes that companies compete against each other to maximise their profits. It is thought that collaboration (cooperation) of companies emerges as a result of the profit maximisation of companies at an individual level.

- Pure and mixed strategies: A pure strategy indicates that a player takes only one choice out of the available strategies. On the other hand, a mixed strategy means that a player takes multiple choices, randomly, with certain probabilities. The pure strategy is thereby a special case of mixed strategy.

- Strategic form: This is a way to define a game in the form of matrix which shows players, strategies, and payoffs. In this case the player stands for an autonomous agent who is able to make a decision; a strategy represents a collection of actions which circumscribes the behaviour of a player in any situation; while payoff indicates an evaluation associated with each action. From now on we will use "player" as a technical term instead of "company". The possible choices for a company about whether to increase or decrease $K_{i}(t)$ and $L_{i}(t)$ constitute the strategies of the agent. In this form all players are assumed to make decisions simultaneously and without knowing other players' choices.

- Best response: This is the strategy which leads to the highest payoff for a player, taking other players' strategies as given.

- Nash equilibrium: This is a set of the strategies corresponding to the best reactions for every player. In such a state, no player will change 
Table 5.1: Strategic form game 1

\begin{tabular}{|c||c|c|}
\hline $\mathrm{A}$ & $\mathrm{B}$ & $\mathrm{T}$ \\
\hline $\mathrm{S}$ & 4,4 & 6,1 \\
\hline $\mathrm{T}$ & 1,6 & 2,2 \\
\hline
\end{tabular}

Table 5.2: Strategic form game 2

\begin{tabular}{|c||c|c|}
\hline \multicolumn{1}{|c||}{$\mathrm{A}$} & $\mathrm{B}$ & $\mathrm{T}$ \\
\hline \hline $\mathrm{S}$ & 4,4 & 1,6 \\
\hline $\mathrm{T}$ & 6,1 & 2,2 \\
\hline
\end{tabular}

his or her strategy unless other players change their strategies. This equilibrium state was named after J. F. Nash, Jr., ${ }^{3}$ who first proposed it.

- Extensive form: This is a form of game in which the decision making of players is repeated as time passes. Such a process is depicted using a tree diagram. Each node of the tree represents a point of choice for a player. Branches growing out of the node represent possible actions for the player.

- Perfect information: This is the most basic game in extensive-form games. A player can select his or her strategy knowing exactly the past behaviours of other players.

- Rationality: This means that a player behaves to maximise his or her own payoff by considering a complete set of possible strategies adopted by other players in the future. On the other hand, bounded rationality means that a player has to consider other players' future strategies to a limited extent and make an optimum decision with imperfect knowledge.

We will explain the basics of game theory by solving three illustrative examples of strategic form games as shown in Tables 5.1, 5.2, and 5.3. In these examples players A and B can select two strategies, $\mathrm{S}$ and $\mathrm{T}$. The left number in each cell of the table refers to the payoff for $\mathrm{A}$ and the right number to that for B.

Let us begin with the very simple game shown in Table 5.1. If player A adopts mixed strategy $\mathrm{S}$ with probability $p$, then he or she adopts the other

\footnotetext{
${ }^{3}$ Nash won the Nobel Prise in Economy in 1994 and was featured in the film "A Beautiful Mind".
} 
Table 5.3: Strategic form game 3

\begin{tabular}{|c||c|c|}
\hline $\mathrm{A}$ & $\mathrm{B}$ & $\mathrm{T}$ \\
\hline $\mathrm{S}$ & 6,4 & 0,0 \\
\hline $\mathrm{T}$ & 0,0 & 4,6 \\
\hline
\end{tabular}

strategy $\mathrm{T}$ with probability $1-p$. The probabilities of player $\mathrm{B}$ adopting strategies $\mathrm{S}$ and $\mathrm{T}$ are given as $q$ and $1-q$, respectively.

According to Table 5.1, the expectation value of payoff for player A is

$$
4 p q+6 p(1-q)+1(1-p) q+2(1-p)(1-q)=(4-q) p-q+2 .
$$

The inequality $0 \leq q \leq 1$ guarantees that the prefactor $(4-q)$ of $p$ is always positive. The more $p$ is increased, therefore, the more payoff player A can obtain. Strategy $\mathrm{S}(p=1)$ is thus the best reaction for player A. The expectation value of payoff for player $\mathrm{B}$ is likewise given as

$$
4 p q+1 p(1-q)+6(1-p) q+2(1-p)(1-q)=(4-p) q-p+2 .
$$

Since $0 \leq p \leq 1$, the prefactor $(4-p)$ of $q$ always takes a positive value and hence strategy $\mathrm{S}(q=1)$ is also the best reaction for player B. From the above-mentioned arguments, a set of strategies $(\mathrm{S}, \mathrm{S})$ gives a Nash equilibrium solution.

The next example is defined by Table 5.2. The payoffs are slightly different from those in Table 5.1, and the example manifests an interesting phenomenon known as prisoner's dilemma.

The expectation value of payoff for player $\mathrm{A}$ is calculated as

$$
4 p q+1 p(1-q)+6(1-p) q+2(1-p)(1-q)=-(1+q) p+4 q+2
$$

The inequality $0 \leq q \leq 1$ proves that the prefactor $-(1+q)$ of $p$ is definitely negative. The payoff of player $\mathrm{A}$ increases with decreased $p$, so the best reaction for that player is strategy $\mathrm{T}(p=0)$.

The expectation value of payoff for player B is also calculated as

$$
4 p q+6 p(1-q)+1(1-p) q+2(1-p)(1-q)=-(1+p) q+4 p+2 .
$$

The prefactor $-(1+p)$ is likewise negative. Hence the best reaction for player $\mathrm{B}$ is the same as that for player A. We thus see that a set $(\mathrm{T}, \mathrm{T})$ of strategies is in Nash equilibrium. Let us look at the payoffs in Table 5.2 again. We find the strategy pair $(\mathrm{S}, \mathrm{S})$ gives a better choice for both of the players than the Nash equilibrium solution $(\mathrm{T}, \mathrm{T})$. Such a phenomenon, called prisoner's dilemma, is a typical example of the way that a combination of the best strategies for individual players does not always result in global optimisation. 


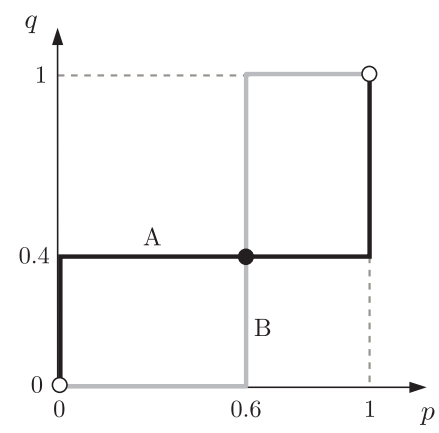

Figure 5.24: Multiple Nash equilibrium solutions

The two games so far examined have only one Nash equilibrium solution, but the last game in Table 5.3 has many. The expectation value of payoff for player $\mathrm{A}$ is

$$
6 p q+4(1-p)(1-q)=(10 q-4) p-4 q+4 .
$$

Here the positivity of the prefactor $(10 q-4)$ of $p$ is not guaranteed by $0 \leq$ $q \leq 1$. If $(10 q-4)$ is positive $(q>0.4)$, the best reaction for player $\mathrm{A}$ is strategy $\mathrm{S}(p=1)$. If $(10 q-4)$ is negative $(q<0.4)$, on the other hand, the best reaction is strategy $\mathrm{T}(p=0)$. In addition to these two cases, if $(10 q-4)$ is equal to zero $(q=0.4)$, player A can select any strategy with $0 \leq p \leq 1$. The expectation value of payoff for player $\mathrm{B}$ is given as

$$
4 p q+6(1-p)(1-q)=(10 p-6) q-6 p+6 .
$$

Likewise the prefactor $(10 p-6)$ of $q$ takes either positive or negative values. Therefore, the best reaction for player B is strategy $\mathrm{S}(\mathrm{T})$ for $p>0.6(p<0.6)$ and any strategy with $0 \leq q \leq 1$ at $p=0.6$.

The tracks of the best reactions for players A and B are overlaid on the $p$ - $q$ plane as shown in Fig 5.24. The intersections of the two lines depicted by open and filled circles identify Nash equilibria. Note that there are three such solutions. The open circles corresponding to $(\mathrm{S}, \mathrm{S})$ and $(\mathrm{T}, \mathrm{T})$ are solutions for pure strategies and the filled circles at $(p, q)=(0.6,0.4)$ is a solution for mixed strategies.

The three examples discussed so far are all games in which each player makes a single decision. However managers of actual companies have to make multiple decisions over extended periods of time. This situation corresponds to an extensive form game, which can be graphically represented by way of a game tree diagram. Here we suppose that payoffs of players are calculated in a finite period of time and also they play with perfect information. In a game of perfect information represented by a tree of finite size we can obtain the exact solution for a pure strategy Nash equilibrium using backward induction. 
Here is an illustrative example with two terms as shown in Figure 5.25. Players A and B take strategy $\mathrm{S}$ or $\mathrm{T}$, and player B makes a decision after player A. Since this is a game of perfect information, each of the information sets depicted by open circles has only a single branching point. The rightmost end points are accompanied by the vectors of payoffs. For instance, let us concentrate on the case where players $\mathrm{A}$ and $\mathrm{B}$ select strategies $\mathrm{S}$ and $\mathrm{T}$, respectively, in the first term, and then take $\mathrm{T}$ and $\mathrm{S}$, respectively, in the second term. This series of actions assigns payoffs $(7,10)$ to the players A and B. Those payoff values should be regarded as what results when the calculation takes into account interactions between agents. Backward induction proceeds in a direction from end to root by choosing either of the upper and lower branches whose payoff is larger. We first decide the strategy of player B in the second term. In this example the payoffs of the upper branches (strategy $\mathrm{S}$ ) are always larger than those of the lower branches (strategy T) for player B, so that player B takes strategy $\mathrm{S}$ in the second term; this selection is depicted by solid lines in Figure 5.25. Then player A takes strategy $\mathrm{T}$ for all branches in the second term because the lower branches have payoffs larger than the upper branches; this selection is also depicted by solid lines in Figure 5.25. In the next step, player B and then player A decide their strategies in the first term. The decision is made exactly in the same way as that in the second term. The iteration of this procedure enables us to find a Nash equilibrium, which is shown by a connected thick line. We remark that it is not necessarily the case that a player selects the same strategy at every stage in each term, although it is true for this example; the choice of strategies depends on their payoff values.

\subsubsection{Model of Bounded Rationality}

The number of branches in an extensive game grows exponentially as the numbers of players and terms increase. Accordingly, the computational task based on backward induction becomes heavier, requiring a larger memory region and longer computing time. When carrying out simulations for a system with many agents, we thereby need an alternative method. One of the candidates is a genetic algorithm (Goldberg, 1989) which finds an approximate solution to the Nash equilibrium efficiently with less intensive use of computing resources, thus enabling us to carry out simulations for a large-scale system.

The genetic algorithm is an heuristic optimisation method, and the computational procedure consists of nine steps as outlined below.

- Step 1: We input $N$ agents, $T$ terms, $M$ genes, $L$ generations, the probability $p_{c}$ of crossover, and the probability $p_{m}$ of mutation. Here the gene is a long tape of the type shown in Figure 5.26. Typical values for $p_{c}$ and $p_{m}$ are 0.8 , and the inverse of the length of genes, respectively; however, these values may have be tuned in the light of emergent problems. Each gene site (a separated sector on the tape) inscribes the 


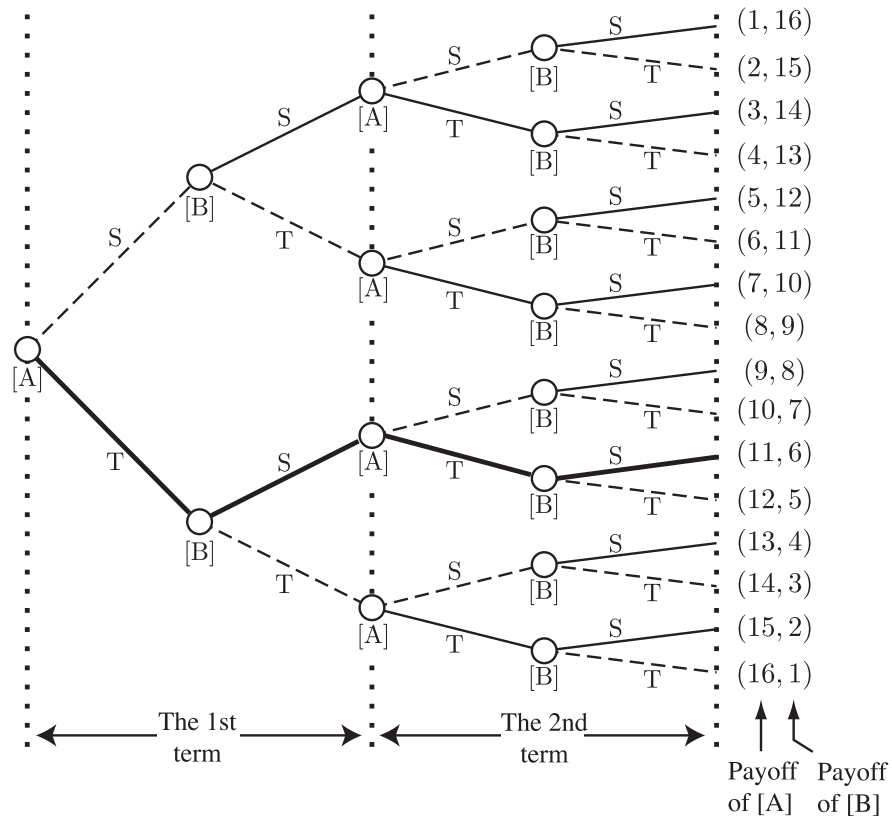

Figure 5.25: Extensive form game 


\begin{tabular}{|l|l|l|l|l|l}
\hline$\pm \Delta K_{1}(0)$ & $\pm \Delta L_{1}(0)$ & $\ldots$ & $\pm \Delta K_{1}(T-1)$ & $\pm \Delta L_{1}(T-1)$ & $\ldots$ \\
\hline
\end{tabular}

\begin{tabular}{l|l|l|l|l|l|}
\hline$\cdots$ & $\pm \Delta K_{N}(0)$ & $\pm \Delta L_{N}(0)$ & $\cdots$ & $\pm \Delta K_{N}(T-1)$ & $\pm \Delta L_{N}(T-1)$ \\
\hline
\end{tabular}

Figure 5.26: Gene

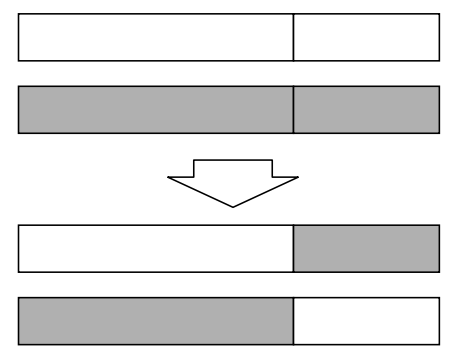

Figure 5.27: Crossover process

strategy of each agent. In the $t$-th period the $i$-th agent may increase or decrease the capital $K_{i}(t)$ and the labour $L_{i}(t)$; that is to say in total it has four strategies. In Figure 5.26, $+\Delta K_{i}(t)$ is set to 1 when $K_{i}(t)$ is increased and $-\Delta K_{i}(t)$ to 0 when it is decreased. The same is true for $L_{i}(t)$.

- Step 2: We generate initial values for $M$ genes; 1 or 0 is written randomly on each gene site.

- Step 3: For each gene, we select another gene randomly with the probability $p_{c}$. As displayed in Figure 5.27, the two genes are then cut at a position randomly determined and the two portions are exchanged. This is called a crossover process.

- Step 4: For every gene site, the value is flipped with the probability $p_{m}$; 0 is converted to 1 or vice versa. This, called a mutation process, and is demonstrated in Fig 5.28.

- Step 5: For the $j$-th gene, we calculate the total sum of profits made by the $i$-th company during the period $(0, T)$; the payoff is denoted by $P_{i}^{(j)}$. Then we calculate the payoffs of all of the companies $(i=1, \ldots, N)$ for 


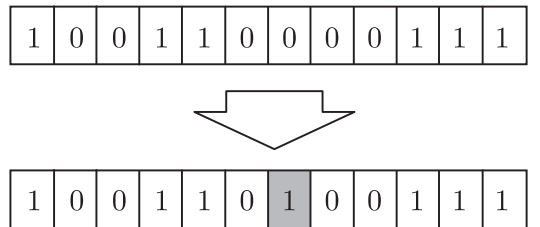

Figure 5.28: Mutation process

\section{[Payoff]}

\begin{tabular}{|c|c|c|c|c|c|c|c|c|}
\hline$P_{1}^{(1)}$ & $P_{2}^{(1)}$ & $P_{3}^{(1)}$ & $P_{4}^{(1)}$ & $\cdots$ & $P_{N-3}^{(1)}$ & $P_{N-2}^{(1)}$ & $P_{N-1}^{(1)}$ & $P_{N}^{(1)}$ \\
\hline$P_{1}^{(2)}$ & $P_{2}^{(2)}$ & $P_{3}^{(2)}$ & $P_{4}^{(2)}$ & . & $P_{N-3}^{(2)}$ & $P_{N-2}^{(2)}$ & $P_{N-1}^{(2)}$ & $P_{N}^{(2)}$ \\
\hline & & & & & & & & \\
\hline$P_{1}^{(M)}$ & $P_{2}^{(M)}$ & $P_{3}^{(M)}$ & $P_{4}^{(M)}$ & 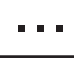 & $P_{N-3}^{(M)}$ & $P_{N-2}^{(M)}$ & $P_{N-1}^{(M)}$ & $P_{N}^{(M)}$ \\
\hline \multicolumn{9}{|c|}{ [Rank] } \\
\hline 8 & 26 & 5 & 7 & 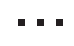 & 2 & 21 & 3 & 4 \\
\hline$M-6$ & 1 & $M-1$ & 31 & - & 5 & $M-3$ & 1 & 6 \\
\hline & & & & & & & & \\
\hline 6 & 3 & 2 & $M-1$ & $\cdots$ & 8 & $M$ & 15 & $M$ \\
\hline
\end{tabular}

Figure 5.29: Payoff and fitness

all of the genes $(j=1, \ldots, M)$. Here we note that the interactions between agents are taken into account in the calculation of the profits.

- Step 6: We calculate the individual fitness for $M$ genes. As shown in Figure 5.29, the $M$ genes are ranked for each site. The figure shows that the first agent is ranked eighth in the first gene, $(M-6)$-th in the second gene, ..., sixth in the $M$-th gene. Then we sum up the ranking values assigned at every site for each gene and define the inverse of the sum as fitness of the gene. If a gene is ranked first at all sites, its fitness is equal to $1 / N$, the maximum value that the fitness can take. Since the strategies are best optimised for all of the agents in this case, we have the Nash equilibrium solution.

- Step 7: Using random numbers, we select $M$ successors from the $M$ 


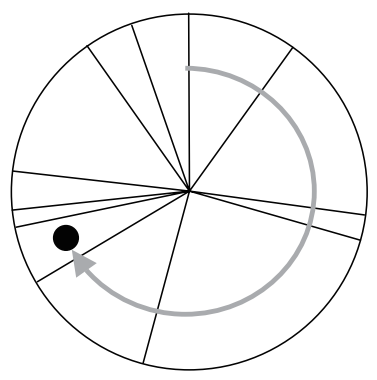

Figure 5.30: Selection by roulette method

genes according to their fitness values, where selection of the same genes is allowed. This selection amounts to $M$ repetitions of such a roulette game as visualised in Figure 5.30. The roulette is made with $M$ selections corresponding to the $M$ genes and each area of the selections is proportional to the fitness value of the corresponding gene.

- Step 8: We repeat the procedure from step 3 through the step 7 by $L$ times.

- Step 9: The genetic information with the highest fitness score is recorded.

A Nash equilibrium solution obtained by a genetic algorithm is different in two ways from that based on backward induction. We first note that the genetic algorithm gives an approximate solution to the Nash equilibrium, not the exact solution. This is true for many complicated problems. Therefore the solution is best understood as decision making by an agent with bounded rationality. Secondly, each agent makes its decision without knowing strategies adopted by others. This amounts to not assuming that agents possess perfect information.

The agent-based model sketched here serves as a starting point for building a model of autonomous agents interacting on a transaction network. We believe that this kind of model can and should play an important role in the exploration of business applications, and in the next chapter this perspective will be explored regarding the possible application of econophysics to the management of companies in the real economy, with illustrative applications of the present model in Sections 6.1.4 and 6.2.6. 


\section{Chapter 6}

\section{Perspectives for Practical Applications}

In the previous chapters, we have described various outcomes derived from the basic research, such as a growth theory of companies, business networks, and agent simulations. In this chapter, by changing our perspective, we will review what consequences are expected from the research outcomes, in other words the practical applications of Econophysics. Real economic data is abundant in Japan compared with other countries, and for this reason alone the Japanese economy is of particular importance in our work. It is also convenient in other ways. The practical applications of our work are currently under development, and the concrete content of these applications is being improved through ongoing conversations with companies in Japan. Indeed, the contents of this chapter should be regarded as an interim statement, and might be greatly altered in the future. However, we think that it is of real interest even at this stage to describe some of our suggested applications. We will concentrate on three topics, namely, the methodology for developing a business strategy, the management of the propagation of credit risk, and the encouragement of innovation in business models, and offer them as of potential value to central government civil servants, acquisition specialists, investment bankers, staff working in the management planning division of companies, financial departments, materials departments, credit divisions, and rating companies, as well as business administrators.

\subsection{Development of Business Strategies}

In this section we will begin by explaining the elements of corporate finance theory, then move on to discuss issues relating to the practical applications of that theory. Subsequently, we will describe a simulation of company decision making and business performance in a competitive environment under a given macroeconomic trend using the autonomous agent-based model. This 
approach is not considered in traditional corporate finance theory.

\subsubsection{Valuation of Companies}

We need to understand the elements of corporate finance theory in order to estimate corporate value (Brealey et al., 2008). Corporate finance starts by reading financial statement data, such as income statements, balance sheets, and cash flow statements. Although it is a hard work to read financial statement data in detail, it is easy to understand their outlines, so a description of these fundamentals is the first step towards an understanding of corporate valuation.

When examining a company's operation, sales revenue is decomposed into the following three components:

$$
\text { Sales Revenue }=\text { COGS }+ \text { SGA }+ \text { Operating Profit, }
$$

where COGS is the abbreviated form of Cost of Goods Sold, and SGA stands for Selling, General and Administrative expenses. COGS is the sum of three costs, that is the cost of raw materials, labour costs, and the amortisation cost of premises and equipment. SGA consists of the employment costs of the sales department and administration staff and all sorts of other costs. By rewriting the above relation we can obtain the operating profit,

$$
\text { Operating Profit }=\text { Sales Revenue }- \text { COGS }- \text { SGA } .
$$

Next we calculate the free cash flow from the operating profit. The cash flow, which literally means the flow of money, is a fundamental quantity in corporate valuation, and, more specifically, the net cash flow generated from a company's business operation is called the free cash flow (FCF) and is the sum of the operating cash flow and the investment cash flow. It is noted that the sign of cash flow is deemed to be positive when cash flows to the company from other companies, and to be negative when cash flows from the company to other companies. In other words, free cash flow is the money remaining when expenses incurred in a company's operation are deducted from earnings. The word "free" indicates that a business administrator can distribute this cash flow freely to investors in the company. This quantity is estimated approximately by using both the income statement and the company balance sheet:

$$
\begin{aligned}
& \text { Free Cash Flow } \\
&= \text { NOPAT }+ \text { Amortisation Cost of Premises and Equipment } \\
& \text { - Investment of Premises and Equipment } \\
& \text { - Increasing Working Capital, }
\end{aligned}
$$

where NOPAT stands for Net Operating Profit After Tax, although the exact value of free cash flow is usually reported in a cash flow statement with decomposition into two components:

Free Cash Flow $=$ Operating Cash Flow + Investment Cash Flow. 
Table 6.1: Correspondence between symbols used in corporate finance theory and variables in this book

\begin{tabular}{|c|c|c|}
\hline Name of quantity & Corporate finance theory & This book \\
\hline \hline $\begin{array}{c}\text { Free Cash Flow } \\
\text { after tax }\end{array}$ & FCF & $C_{t}$ \\
\hline Economic profit & NOPAT & $O_{t}$ \\
\hline Corporate value & EP & $R_{t}$ \\
\hline Net present value & NPV & $U$ \\
\hline $\begin{array}{c}\text { Weighted average } \\
\text { cost of capital }\end{array}$ & WACC & $r_{w}$ \\
\hline Internal rate of return & IRR & $r_{0}$ \\
\hline
\end{tabular}

The correspondence relations of symbols used in corporate finance theory and variables in this book are summarised in Table 6.1.

The corporate value $(\mathrm{CV}) U$ at the present time $(t=0)$ is calculated from the time series of the expected future free cash flow $C_{t}(t=1, \ldots, T)$ according to

$$
\begin{gathered}
U=\sum_{t=1}^{T} \frac{C_{t}}{\left(1+r_{w}\right)^{t}}, \\
r_{w}=r_{e} \frac{E}{E+D}+r_{d}(1+\tau) \frac{D}{E+D},
\end{gathered}
$$

where $\left(1+r_{w}\right)^{t}$ in the denominator is a factor to reduce $C_{t}$ for the corresponding value at $t=0$, namely the present value. The weighted average cost of capital (WACC) $r_{w}$ is sometimes called the discount rate for short. In addition, $r_{e}, r_{d}, E, D, \tau$ are the returns on invested capital, borrowing rate, invested capital, debt payable, and tax rate, respectively. The borrowing rate $r_{d}$, the invested capital $E$, and debt payable $D$, are recorded for each company in the relevant financial statements. The expected rate of return $\mu$, which is calculated by using the historical series of stock prices with the capital asset pricing model (CAPM), is used as a typical value for the return on invested capital $r_{e}$ relating the companies listed in the stocks market.

The corporate value $U$ is also defined as the sum of the present value of economic profit $R$,

$$
\begin{gathered}
U=\sum_{t=1}^{T} \frac{R_{t}}{\left(1+r_{w}\right)^{t}}+E+D, \\
R_{t}=O_{t}-r_{w}(E+D),
\end{gathered}
$$


The quantity $V$, calculated by deducting invested capital $E+D$ from corporate value $U$, is referred to as the net present value (NPV):

$$
V\left(r_{w}\right)=U\left(r_{w}\right)-E-D
$$

which plays a very important role in business investment theory for decision making. If the NPV of the business under consideration is positive, the business will be profitable, and the business is suitable for investment. If $r_{w}$ is chosen so that $V\left(r_{w}\right)$ is equal to zero:

$$
V\left(r_{0}\right)=0
$$

the discount rate $r_{0}$ is called the internal rate of return (IRR).

\section{$\alpha_{\beta}^{\gamma}$ Capital asset pricing model}

In what follows we will try to explain the basics of the Capital Asset Pricing Model (CAPM) (Luenberger, 1997). The return $r_{i}(t)$ of the risk asset $i$ changes from time to time. In CAPM the expected rate of return $\mu_{i}=\left\langle r_{i}\right\rangle$ of the risk asset $i$ is given by

$$
\mu_{i}=r_{f}+\beta_{i}\left(\mu_{M}-r_{f}\right),
$$

where $r_{f}$ and $\mu_{M}$ are, respectively, the return of a risk-free asset and the expected rate of return of the market portfolio. The coefficient $\beta_{i}$ is a proportional factor multiplied by the risk premium $\mu_{M}-r_{f}$, and is given by,

$$
\beta_{i}=\sigma_{i M} / \sigma_{M}^{2},
$$

where $\sigma_{i M}$ is the covariance (see the column on p. 121) between the return on the market portfolio and return of the asset $i$.

Let us assume that the portfolio consists of risk assets $i(=1, \ldots, n)$ with a ratio $x_{i}$. Thus we have $\sum_{i=1}^{n} x_{i}=1$. The expected rate $\mu_{p}$ of return and the variance $\sigma_{p}^{2}$ of the return for this portfolio are written as

$$
\begin{gathered}
\mu_{p}=\sum_{i=1}^{n} x_{i} \mu_{i}, \\
\sigma_{p}^{2}=\sum_{i=1}^{n} \sum_{j=1}^{n} x_{i} x_{j} \sigma_{i j},
\end{gathered}
$$

where $\sigma_{i j}$ is the covariance between assets $i$ and $j$. Minimising the variance $\sigma_{p}^{2}$ with respect to variables $x_{i}(i=1, \ldots, n)$ is called portfolio optimisation.

We will now assume that there is no correlation between the returns from the $\operatorname{assets}\left(\sigma_{i j}=0, i \neq j\right)$. The variance of the return of the risk asset $i$ is

$$
\sigma_{i}^{2}=\beta_{i}^{2} \sigma_{M}^{2}+\tilde{\sigma}_{i}^{2},
$$


and thus the variance of the return of the portfolio $\sigma_{p}^{2}$ is written as,

$$
\sigma_{p}^{2}=\sum_{i=1}^{n} x_{i}^{2}\left(\beta_{i}^{2} \sigma_{M}^{2}+\tilde{\sigma}_{i}^{2}\right) .
$$

The first term and the second term of the r.h.s. of Eq. (6.1) are the market risk and the idiosyncratic risk, respectively. By assuming $x_{i}=1 / N$, the limit $N \rightarrow \infty$ of the second term of r.h.s. of Eq. (6.2) converges to zero. We could set up the portfolio without idiosyncratic risk by combining a large number of risk assets. It is noted that only market risk brings profit, because $\beta_{i}$ is included only in risk. It was recently discovered that the major fraction of the covariance matrix $\sigma_{i j}$ consists of noise, and noise reduction methodology using the random matrix theory is attracting the attention not only of academic researchers but also of those working in practical business situations.

\subsubsection{Optimum Capital Structure}

The capital structure of a company is characterised by the ratio of invested capital $E$ and debt payable $D$. In economics there is a well-known theory regarding capital structure, namely the Modigliani-Miller theorem (Modigliani and Miller, 1958). In this theorem, by assuming a perfect capital market, it is shown that corporate value is independent of the company's capital structure. However the above assumption is not valid in the real economic system, thus it is expected that in practice there is an optimum capital structure for the maximisation of corporate value.

There are various methods to determine the optimum capital structure, one, for example, calculates a trade-off between opportunity loss and bankruptcy cost, while another proposes a structure designed to minimise agency cost. An alternative method approaches the problem by minimising the cost of financing.

In the following example, the optimum structure is determined from the point of view of risk management as shown in Figure 6.1. When events of corporate value $U$ are generated using a Monte-Carlo simulation about a certain business in due diligence process, we obtain the distribution of $U-D$ by subtracting the debt payable $D$ from the generated corporate value $U$. If $U-D$ is negative without invested capital $E$, the company immediately goes bankrupt. On the other hand, if the company is rich in invested capital $E$ to cover the negative deficit $U-D$, the company does not go bankrupt. Thus it is possible to determine the invested capital $E$ required in order to avoid bankruptcy for the large fluctuation of the deficit $U-D$, under the condition that the average net present value is positive $\langle V\rangle>0$. The invested capital $E$ determined by this method is exposed to risk, that is the fluctuation of cash flow. Therefore the invested capital $E$ determined above is called the risk capital $E_{p}$. 
210 CHAPTER 6. PERSPECTIVES FOR PRACTICAL APPLICATIONS

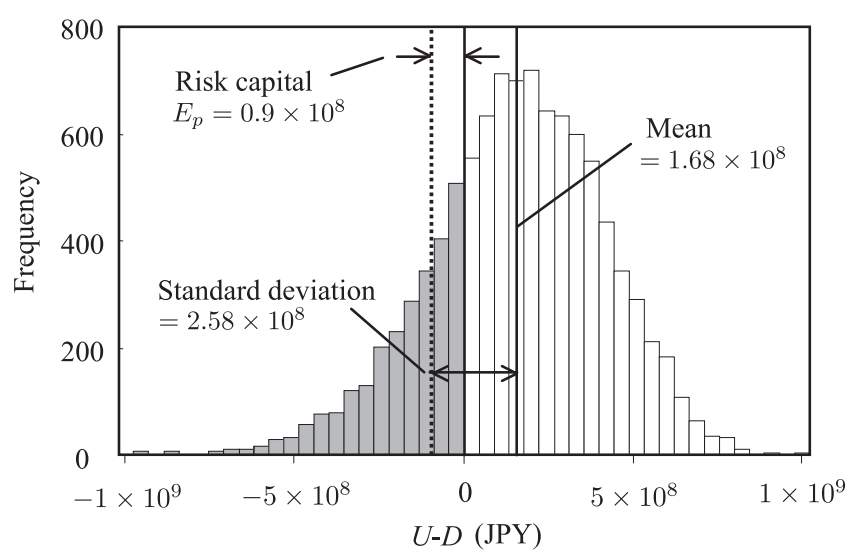

Figure 6.1: Distribution of $U-D$ and risk capital $E_{p}$

\section{$\alpha_{\beta}^{\gamma}$ Modigliani and Miller theorem}

Suppose the capital structure of company A is different from that of company B, e.g. company A has only equity as invested capital, and company B has both equity and bonds. The question is which company's market value is larger than the other's.

The earnings before interest, the market values of stocks and bonds for the company $i(=\mathrm{A}, \mathrm{B})$ are $X_{i}(=X), S_{i}$, and $D_{i}\left(D_{\mathrm{A}}=0\right)$, respectively. Thus the market values for the companies $\mathrm{A}$ and $\mathrm{B}$ are

$$
\begin{gathered}
V_{\mathrm{A}}=S_{\mathrm{A}}, \\
V_{\mathrm{B}}=S_{\mathrm{B}}+D_{\mathrm{B}} .
\end{gathered}
$$

We have here assumed that the complete capital market satisfies the following conditions:

- There is no tax and no bankruptcy.

- No cost is required for issuance and trading of stocks and bonds.

- Investors can borrow any amount of money with an interest rate equal to that of company B. (Company A has only equity.)

- The differing stakes among financial institutes, companies, and investors are harmonised without cost.

Initially, we will assume that the investor's portfolio consists of only stock $\alpha_{\mathrm{B}} S_{\mathrm{B}}$. The coefficient $\alpha_{i}$ is the fraction of equity of the company $i$ owned by the investor $\left(0<\alpha_{i}<1\right)$. The profit of the portfolio is

$$
Y_{\mathrm{B}}=\alpha_{\mathrm{B}}\left(X-r D_{\mathrm{B}}\right),
$$


where $r$ is an interest rate of the company B.

If the stock of the company $\mathrm{A}$ is comparatively low in price, $\left(V_{\mathrm{A}}<V_{\mathrm{B}}\right)$, the investors will sell the stock $\alpha_{\mathrm{B}} S_{\mathrm{B}}$, and buy the stock of company $\mathrm{A}, \alpha_{\mathrm{A}} S_{\mathrm{A}}$, by borrowing $d=\alpha_{\mathrm{B}} D_{\mathrm{B}}$. Here it is noted that $\alpha_{\mathrm{A}}=\left(\alpha_{\mathrm{B}} S_{\mathrm{B}}+d\right) / S_{\mathrm{A}}$. In this case, the profit of the portfolio is equal to

$$
Y_{\mathrm{A}}=\alpha \frac{V_{\mathrm{B}}}{V_{\mathrm{A}}} X-r \alpha D_{\mathrm{B}} .
$$

If $V_{\mathrm{A}}<V_{\mathrm{B}}$, we have the relation $Y_{\mathrm{A}}>Y_{\mathrm{B}}$ by comparing Eq. (6.3) and Eq. (6.4). Thus other investors with stock $\alpha_{\mathrm{B}} S_{\mathrm{B}}$ trade in exactly the way described above until the relation $V_{\mathrm{A}}=V_{\mathrm{B}}$ is obtained.

Now, let us consider an investor's portfolio consisting of only stock $\alpha_{\mathrm{A}} S_{\mathrm{A}}$. The profit of the portfolio is

$$
Y_{\mathrm{A}}^{\prime}=\alpha_{\mathrm{A}} X
$$

If the stock of the company $\mathrm{B}$ is comparatively low in price, $\left(V_{\mathrm{A}}>V_{\mathrm{B}}\right)$, the investor will sell the stock, $\alpha_{\mathrm{A}} S_{\mathrm{A}}$, and buy the stock of company $\mathrm{B}$ by $\alpha_{\mathrm{B}} S_{\mathrm{B}}=S_{\mathrm{B}} \alpha_{\mathrm{A}} S_{\mathrm{A}} / V_{\mathrm{B}}$ and the bonds of company $\mathrm{B}$ by $\beta_{\mathrm{B}} D_{\mathrm{B}}=D_{\mathrm{B}} \alpha_{\mathrm{A}} S_{\mathrm{A}} / V_{\mathrm{B}}$. Here $\beta_{i}$ is the fraction of the bonds of company $i$ owned by the investor. It is noted that $\alpha_{\mathrm{B}} S_{\mathrm{B}}+\beta_{\mathrm{B}} D_{\mathrm{B}}=\alpha_{\mathrm{A}} S_{\mathrm{A}}$. In this case, the profit of the portfolio is equal to

$$
Y_{\mathrm{B}}^{\prime}=\alpha_{\mathrm{A}} \frac{V_{\mathrm{A}}}{V_{\mathrm{B}}} X .
$$

If $V_{\mathrm{A}}>V_{\mathrm{B}}$, we have the relation $Y_{\mathrm{A}}^{\prime}<Y_{\mathrm{B}}^{\prime}$ by comparing Eq. (6.5) and Eq. (6.6). Thus other investors with stock $\alpha_{\mathrm{A}} S_{\mathrm{A}}$ will trade in the way described above until the relation $V_{\mathrm{A}}=V_{\mathrm{B}}$ is obtained.

From the above discussion we can see that the theorem states that a company cannot change its market value $V$ by changing its capital structure, that is the ratio of stock $S$ and debt $E$ (Brealey et al., 2008). This theorem, called the Modigliani and Miller theorem, is said to have been shocking to those engaged in finance, and to have caused considerable controversy, as well it might. In actual situations, however, the financial department of a company is responsible for the maximisation of market value $V$ through financing.

\subsubsection{Decision-Making for Business Entry and Exit}

We will explain decision-making for business entry and exit using the net present value $V$ calculated by the method described in the previous section. If $V>0$ we decide to enter into business or continue the business, while if $V<0$ we will decide not to enter, or to exit from the business. Clearly, the returns from equity $r_{e}$ have to be evaluated by taking business risks properly into account. However we have to recall the fact that any evaluation methodology is only an approximation, thus we cannot evaluate the return on equity precisely.

Consequently, the internal rate $r_{0}$ of return, the rate at which $V=0$ is obtained, is usually used instead. In this case, the decision-making is guided by a comparison of the internal rate of return $r_{0}$ and the target return $r_{h}$. 
If $r_{0}>r_{h}$, we would decide to enter into or continue with the business. If $r_{0}<r_{h}$, we would decide not to enter the business, or to exit.

So far we have explained the theoretical basics of corporate finance, and we can now try to apply these methodologies to real problems. Although the evaluation of corporate value $V$ seems to be straightforward, as explained above, there are many difficulties aside from the discount rate. For instance, we might want to forecast the future revenue required for the evaluation of corporate value. The simplest forecast method is to take the expected market size and multiply it by a target share, where the expected market size is a forecast made by a market research company. In this method, the following four issues are immediately cited:

- Issue 1: When the deviation of future trends in economic growth are taken into account, are the expected market size and the target share considered to be a single scenario?

- Issue 2: How many scenarios for the expected market size and the target share should we consider? How could we estimate deviation for each scenario?

- Issue 3: When the future business scenario deviates very widely, how do we make flexible decision-making other than the initial investment, such as additional investment to extend the business or to sell out of existing business?

- Issue 4: If we make additional investments to extend the business or to sell out of the existing business, what kind of action will its competitor take in response? How could we make a quantitative estimate of the effect of the competitor's action on our sales revenue and profit?

We discuss appropriate actions for each of the issues raised above in turn:

- Discussion 1: Decision-making for multiple scenarios is conducted using the decision tree. The probabilities of scenario bifurcations have to be given by the decision-maker as input parameters.

- Discussion 2: If the number of scenarios to be considered is very large, the scenarios are modeled as a stochastic process and simulated by a Monte Carlo method. The width of deviation of a scenario has to be given by the decision-maker as one of the input parameters. The width of deviation could be estimated by analysing the past financial statements for any companies equivalent to the business under consideration.

- Discussion 3: The effect of stochastic deviation of a business scenario on decision-making can be handled using the real option, that is to say a business valuation method based on an analogy between business decision-making and derivative financial instruments. In this method, the business value is estimated as the price of the derivative financial instrument, where its underlying asset is the cash flow of the business. The 
financial market is assumed to be complete in Economics and Financial Engineering. The pricing of a European-type option in the complete financial market is given by the Black-Scholes formulae, which is an analytic solution of the Black-Scholes partial differential equation (Black and Scholes, 1973). However, firstly, real options do not satisfy the condition of the complete market, secondly the nature of decision-making in business is of American-type rather than European-type. Thus BlackScholes formulae is not appropriate in general for business evaluation.

- Discussion 4: Decision-making under a competitive environment is a very difficult topic to discuss. Indeed, there are still many questions about the quantitative evaluation of the effect of a competitor's action on a company's performance by taking into account transactions between customers and venders. In order to treat this difficult task we have offered and explained a new methodology, that is the transaction network model, in section 5.5.

\section{$\alpha_{\beta}^{\gamma}$ Black-Scholes formulae}

In the following section we will attempt to explain the pricing of a derivative financial instrument with stock as its underlying asset. For a plain European-type call option, the Black-Scholes formulae are used (Hull, 2008). The European-type call option is a claim to buy stock in the company by paying the predetermined exercise price $K$ at the maturity date $T$, if the stock price $S$ is higher than the exercise price $K$ at the maturity date $T$. If the stock price $S$ is higher than the exercise price $K$ at the maturity date $T$, the option holder exercises the claim. The option holder earns a profit $S-K$ by selling the stock at the market price $S$. On the other hand, if the stock price $S$ is lower than the exercise price $K$ at the maturity date $T$, the option holder does not exercise the claim. Therefore the payoff $P$ of the option is

$$
P=\max (S-K, 0)
$$

The stock price $S$ is assumed to be described by the geometric Brownian motion

$$
d S=\mu S d t+\sigma S d z
$$

We obtain the partial differential equation to describe the option price $C=C(S, t)$ at time $t$

$$
\frac{\partial C}{\partial t}+\frac{\sigma^{2} S^{2}}{2} \frac{\partial^{2} C}{\partial S^{2}}+r \frac{\partial C}{\partial S} S-r C=0
$$

using Ito's lemma,

$$
d C=\frac{\partial C}{\partial t} d t+\frac{\partial C}{\partial S} d S+\frac{1}{2} \sigma^{2} S^{2} \frac{\partial^{2} C}{\partial S^{2}} d t
$$

where $r$ is the return on a risk-free asset. Eq. (6.8) is physically interpreted as a drift-diffusion partial differential equation and called the Black-Scholes differential 
equation. This equation can be solved analytically with the boundary condition of the payoff (6.7). The analytic solution given by

$$
\begin{aligned}
& C=S N\left(d_{1}\right)-K e^{-r(T-t)} N\left(d_{2}\right), \\
& d_{1}=\frac{\log \frac{S}{K}+\left(r+\frac{1}{2} \sigma^{2}\right)(T-t)}{\sigma \sqrt{T-t}}, \\
& d_{2}=\frac{\log \frac{S}{K}+\left(r-\frac{1}{2} \sigma^{2}\right)(T-t)}{\sigma \sqrt{T-t}} .
\end{aligned}
$$

is called the Black-Scholes formulae. Here $N(x)$ is the cumulative probability function for the normal distribution. It is noted that the discount rate used in Eq. (6.9) is not the return $\mu$ of stock, but the return on the risk-free asset $r$. This pricing method is called the risk neutral method. For the pricing of the American-type option, which can be exercised before the maturity date $T$, the lattice model is often used (Hull, 2008).

In the next section we will explain a simple application of the model described in the section 5.5.

\subsubsection{Decision-making under a Given Economic Trend}

In principle the macroeconomic trend is determined by aggregating individual company performances. However, at the same time an individual company's investment and performance are affected by the macroeconomic trend. This phenomenon is often called the micro-macro loop, and in this section we will examine the effect of a given macroeconomic trend on an individual company's investment and performance.

The macroeconomic trend is forecast on a periodic basis by many thinktanks, and although these forecasts are not very reliable they can be used practically as possible future scenarios. For this reason it might be invaluable to simulate an individual company's investment and performance under a given macroeconomic trend. This simulation could be conducted by using the transaction network model described in the section 5.5, where the decisionmaking of the company takes into account the interactions between companies due to transactions.

Figure 6.2 shows a portion of the Japanese transaction network. This transaction network shows sixteen listed companies belonging to a Japanese industrial group led by a flagship conglomerate (indicated by open circles), and some 79 listed companies with sales revenue of over 367 billion yen (indicated by filled circles). Even this small portion of the transaction network includes companies with widely varying degree (number of links), for example a small number of the companies have a large degree and many companies have a small degree.

The gross domestic product (GDP) scenario shown in Figure 6.3 is used as 


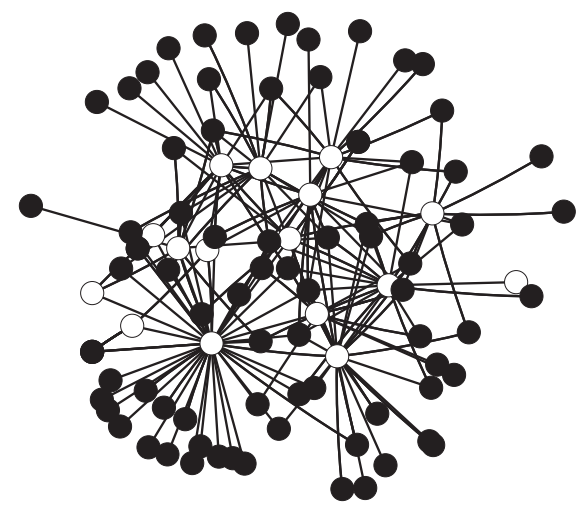

Figure 6.2: Portion of the transaction network in Japan

the macroeconomic trend input for the simulation. Other model parameters were estimated using financial statement data for individual companies during the Japanese Fiscal Year (JFY) 1993 to JFY 2003. Individual company investment and performance under the given macroeconomic trend was then simulated from JFY 1993 to JFY 2003 with the initial values of a company's performance set at JFY 1993. In this case a company's investment means the temporal variation of capital $K$ and labour $L$. A company's performance means the temporal variation of sales revenue $R$ and cost $C$.

Each company makes an investment decision for the capital $K$ and the labour $L$ so that the sum of own operational profit during JFY 1993 and JFY 2003 is maximised. The company's operational profit is determined by sales revenue from customers and cost payment to suppliers, all under the influence of the given macroeconomic trend. Therefore a company's decision-making is the best response to that of another company's strategy, and the obtained results of the simulation are a Nash equilibrium.

In the next stage we will verify the model by comparing the simulation results and the actual data. The cumulative probability distributions of sales revenue at JFY 1993, 1998, and 2003 are shown in Figure 6.4. The open rhombus indicates actual data, and the filled rhombus that of the simulation. The simulation results at JFY 1993 are identical to the actual data because those numbers are initial values, but as time goes by from JFY 1998 to JFY 2003, there is a growing discrepancy between the simulation results and the actual past data. It is however notable that the overall characteristics are similar.

Suppose that an exogenous shock is given at JFY 2003 and the subsequent 


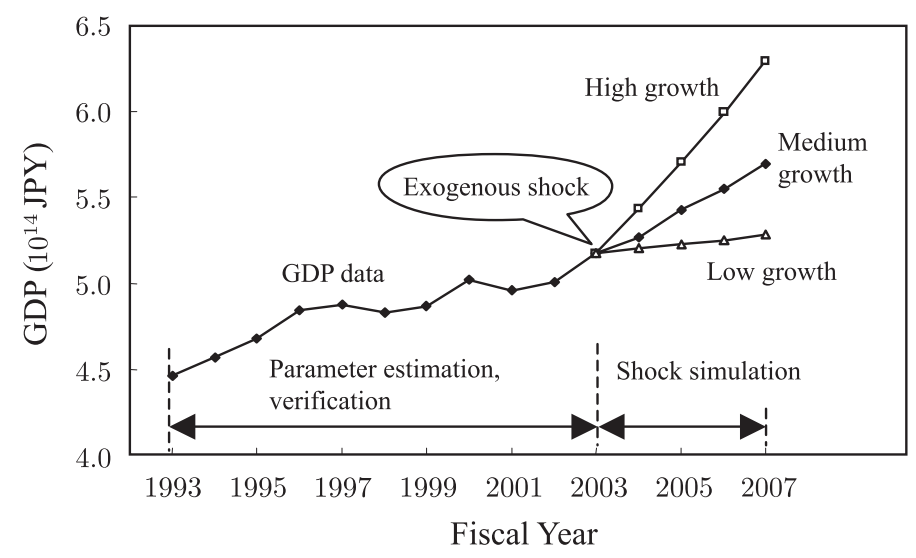

Figure 6.3: GDP scenario

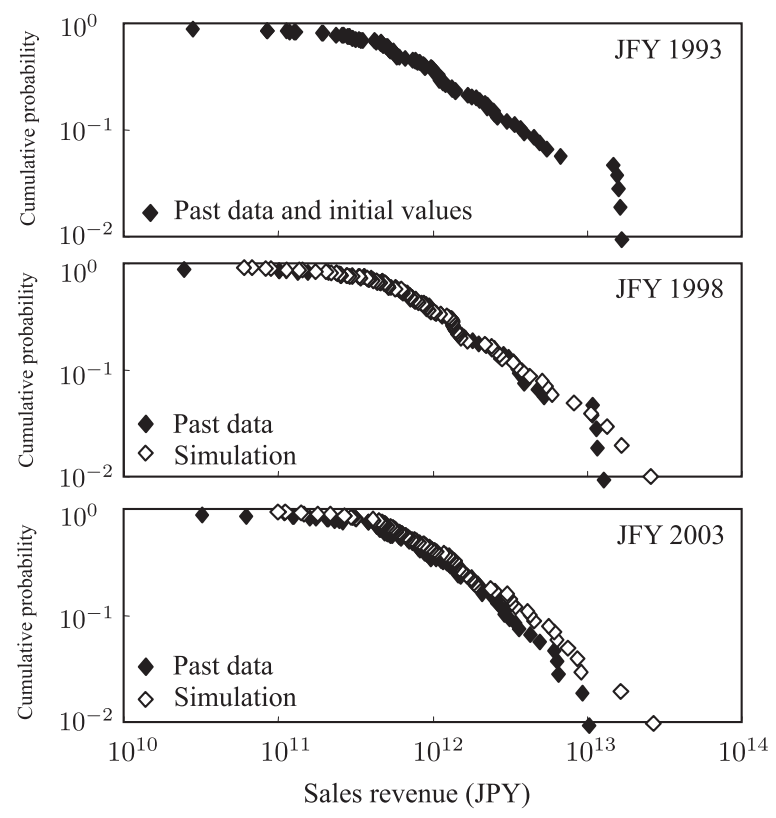

Figure 6.4: Cumulative probability distributions of sales revenues 


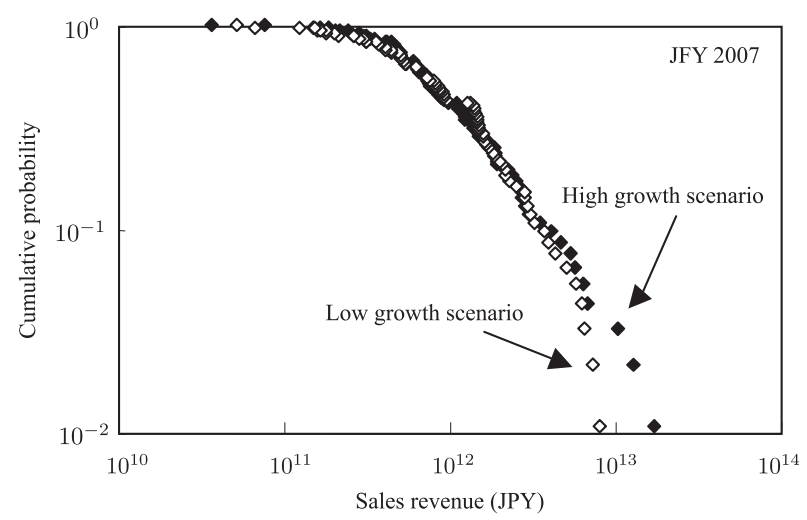

Figure 6.5: GDP scenarios and cumulative probability distributions of sales revenues

GDP trend presents three divergent scenarios, that is, high (5\%), medium $(2.5 \%)$, and low $(0.5 \%)$ growth. We can simulate the effect of these GDP scenarios on the company's investment and performance up to JFY 2007, and Figure 6.5 shows the relation of GDP scenarios and the cumulative probability distributions of sales revenue. The open rhombus indicates the simulation results of the cumulative probability distributions of sales revenue for the low growth scenario, while the closed rhombus plots the results for the high growth scenario. As expected, the high growth scenario gives a distribution with a fatter tail.

So far we have shown the simulation results for the cumulative probability distributions of sales revenue for companies belonging to the transaction network. The temporal variation of the sales revenue $R$, the cost $C$, the capital $K$, and the labour $L$ for an individual company might be used to develop a business strategy. As explained in this section, by using the transaction network model it is possible to simulate an individual company's investment decision-making and resultant performance assuming a particular macroeconomic trend forecast. We believe that this is a significant step towards the practical application of the theories we are proposing.

\subsection{Chain Bankruptcy and Credit Risk}

\subsubsection{Transaction Network}

A transaction network is a succession of economic activities where companies buy materials and intermediate goods and service from suppliers located in the "upstream" area of the network, and add value by making products and services, and sell those to customer companies located in the "downstream" 
area of the network, and to consumers. The exact definition of "upstream" and "downstream" are interesting problems in themselves. We will here content ourselves with the intuitive meaning of these terms, namely that companies manufacturing interim products from crude materials are located upstream, while companies manufacturing end-products from interim products are located downstream. The process by which production successively adds value is extremely complicated. For instance, take the automobile industry. An automobile manufacturer buys various mechanical and electrical parts from auto-parts manufacturers, and manufactures automobiles using machine tools. The resulting manufactured cars have an economic value which exceeds that of the materials and parts.

For example, on the upstream side of the automobile manufacturer there may be an auto-body manufacturer. The auto-body manufacturer buys steel sheets and manufactures auto-bodies using machine tools. The customer companies of the auto-body manufacturer may include not only the above auto-manufacturer but also other auto-manufacturers and even companies in different industry sectors, such as railroad companies. Downstream of the automobile manufacturer we find dealers that sell cars to a large number of consumers located at the outfall of the transaction network; the value added by the dealers is service. So, each individual company buys intermediate goods from upstream companies, and adds value, then sells products to downstream companies and consumers. This cascade of economic activity forms a very complicated network structure with several million Japanese companies engaging in something like 10 million economic relationships.

In complex network theory each company and the relationship through which value is added are called a node and a link, respectively. The whole structure of the succession of economic activity can described by a vast directed graph. Each link consists not only of materials and intermediate goods but also two inputs, namely labour and finance. Most economics textbooks suggest that the production of goods, labour, and finance are the most fundamental processes of economic activity, and this idea is intuitively intelligible if we consider the directed graph to describe the cascade of economic activity. In fact, the goods, labour, and financial markets play a fundamental role in macroeconomics. It is well known that the individual equilibria of each of these three markets are assumed as the grounding concepts of mainstream economics.

Thus, since the aggregation of value added throughout the whole transaction network is equal to the GDP, it is clearly important to understand the structural and temporal variation of the transaction network for any study of the various aspects of macro- and micro-economics. Furthermore, it is also important to understand the meaning of the relative position of each individual company on the transaction network. The economic influence of one company on another is determined by the relative position on the transaction network, because the network structure governs the detailed behaviour of the propagation of both positive and negative economic influences. Since the production of goods requires links between companies, no company can be independent 
of the propagation of both positive and negative economic influence, and it is to this that we will now turn, starting with positive influence.

\section{The Propagation of Positive Economic Influence}

Suppose that consumers and companies on the downstream side of the transaction network make a sizeable demand for a group of final products and for specific intermediate goods. For instance, the promotion of economic growth in China and India increases orders for construction machines such as loading shovels and bulldozers. At this time, a positive economic influence is propagated from the construction machinery manufacturer to upstream companies, for example engine makers selling equipment to the machinery manufacturer. This propagation is repeated from the engine manufacturers to electrical parts manufacturers, and so on.

The range and magnitude of this influence may be strongly dependent on the structure of the transaction network. Although the input-output analysis is aimed at the analysis of the strength of correlation between goods, complex network analysis might reveal the propagation of economic influence through microscopic relationships. In fact, the propagation of economic influence affects production as well as the labour market. There is certainly an influence not only on the labour market but also on financial markets via commercial banks. As can be seen in the foregoing example, the network of companies, financial institutes, and workers plays an essential role in the economic system, but it appears that this network of microscopic relationships is little studied. In the next section we will try to rectify this in part by illustrating the importance of the transaction network by examining the propagation of negative economic influence.

\subsubsection{The Relationship of Debtors and Creditors}

Why does a company manufacture products? Obviously because the company has the expectation of obtaining profits by selling those products. Note that the word "expectation" is complex, and heavily loaded with meanings. Accomplishment of an expectation is not guaranteed a priori, and in this case the profit is determined by sales revenue and cost that are determined a posteriori. Sales revenue depends on demands from customer companies and consumers located in the downstream direction, while, on the other hand, cost depends on supply from suppliers located in the upstream direction, namely labour and financing costs. Consequently, the expected profit is determined a posteriori, depending on the relationship between the upstream and downstream companies.

Basically each link on the transaction network indicates a deal on credit (Stiglitz and Greenwald, 2003). The payment for goods to suppliers is not made concurrently with the delivery of goods, and the payment is often only made after a certain period of time on credit. This payment on credit is referred to as accounts payable by a purchasing company, and accounts re- 
ceivable by a supplier. It should be noted at this point that a purchasing company does not have complete information about the credit status of a supplier. Other examples of payment on credit are finance provided by a bank, and payment of worker wages. Each link in the transaction network is also interpreted as the relationship between a creditor and a debtor, which may bring about the following consequence.

As a company goes into capital deficiency, when its liabilities exceed its capital or has cash-flow problems, the company may go bankrupt. A company located in the upstream region of the bankrupt company might fail to collect accounts receivable, thus if an upstream company goes into capital deficiency, this negative economic influence may propagate further upstream in the network. Consequently many companies may go bankrupt simultaneously; this is a collective phenomenon known as chain bankruptcy.

As explained in the section 3.4, bankruptcy is essentially a cash-flow problem. Even if a company does not go into capital deficit, the deterioration of cash-flow makes operation difficult for the company in many areas, such as production, employment, and finance. In this sense, chain bankruptcy is a serious potential risk not only for an individual company but for the industry as a whole.

Although the loss due to all bankrupt companies exceeds $2 \%$ of the nominal GDP in Japan, as has been demonstrated in Figure 3.20, it might be thought that chain bankruptcy is essentially a rare event and thus that the resulting economic loss is not significant. However, empirical data tells us that this naive view of chain bankruptcy is incorrect. The number of chain bankruptcies occurring in each of the last ten years is about 10 to 20 thousand, as shown in Figure 3.19, and the losses amount to some 10 to 25 trillion JPY, as shown in Figure 3.19. In the next section we will show that chain bankruptcy accounts for about $20 \%$ of the aggregate indebtedness of bankrupt companies, and is a far from negligible economic event (Fujiwara, 2008).

\subsubsection{The Causes of Bankruptcy and the Link Effect}

The data we have analysed was taken from an annual report on business bankruptcy published from the Organisation for Small \& Medium Enterprises and Regional Innovation, JAPAN (SMRJ). This dataset originates from a credit research company, Tokyo Shoko Research, Ltd. (TSR), whose headquarters and branch offices survey bankruptcies with debts more than 10 million JPY, and is all but comprehensive in its coverage of Japanese bankruptcies.

The data categorises the origins of bankruptcy into two main causes, more specifically, the failure in itself and the link effect. ${ }^{1}$

\section{Intrinsic Failure}

${ }^{1}$ TSR categorises each bankruptcy in accordance with the origin of the bankruptcy, which is specified by a score determined by an unpublished method. The origin categorisation is mutually exclusive; although bankruptcies usually have more than one cause, the categorisation is based on the most important one of these. 
(a) Anemic sales (Weak performance)

(b) Irresponsible management (Business fiasco)

(c) Accumulated deficit

(d) Excessively small capital (Lack of working capital, Increase in burden of interest)

(e) Random cause

(f) Build-up of inventory

(g) Excessive business investment

\section{Link Effects}

(a) Aftershock of another company's bankruptcy (excess of bad debts)

(b) Nonenforceable accounts receivable

The remaining origins are 3(a) credit collapse due to suspension of bank transactions, and $3(\mathrm{~b})$ others.

For example, of the 18,246 bankruptcies in JFY 1997, 8,956 were due to (1a), 2,724 to (1b), 2,002 to (1c), 1,792 to (2a), 1,404 to (1d), 373 to (1g), 358 to (2b), 330 to (1e), 210 to (3a), 89 to (1f), and 8 to (3b). The link effect is a negative network effect affecting credit transactions. It works like this: when company $\mathrm{B}$ purchases materials from company $\mathrm{A}$, the transaction is a relation between a lender $(\mathrm{A})$ and a borrower $(\mathrm{B})$. If company B becomes bankrupt then company A might not be able to call in accounts receivable or recover the delivered products. Furthermore, since lender and borrower belong to the same industrial group the relation is rather more complicated than a usual transaction.

The next question is how large the link effect is, and how often it occurs. Whether the effect is dependent on company size is also an interesting question.

\subsubsection{Magnitude of Link Effect}

As shown in Figure 3.21, there were 16,526 bankrupt companies in the calendar year 1997, and the breakdown is: anemic sales (1a) 7,834 companies, link effect (2a) and (2b) 1,942 companies. The figure shows a power law distribution for three orders of magnitude of indebtedness. Figure 6.6 is rank-size plot of indebtedness at bankruptcy plotted to compare the causes for bankruptcy: (1a) and (2). The abscissa is the amount of indebtedness at bankruptcy, and the ordinate is rank of that amount.

Let us compare the probabilities of bankruptcies with two different origins and with an indebtedness between $x$ and $x+d x$. The conditional probability of bankruptcy is equal to the number of bankruptcies with two origins divided by the total number of bankruptcies, thus a comparison with rank-size plots is appropriate. Figure 6.6 shows that the shape of the distribution is fat-tailed for both origins, anemic sales and link effects. It is notable that the slopes of the rank-size plots, that is the power-exponents, are different. The plot for 


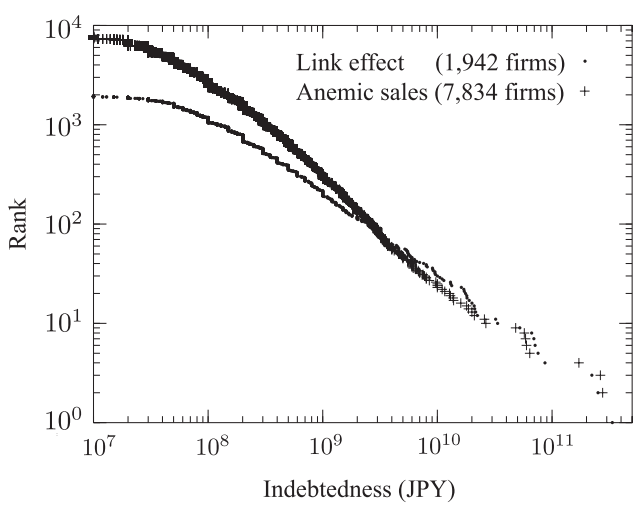

Figure 6.6: Rank-size plots of indebtedness at bankruptcy, comparing origins of bankruptcy

link effect exhibits a fatter tail than the plot for anemic sales, and therefore as indebtedness $x$ increases the conditional probability of bankruptcy due to a link effect becomes larger than that for anemic sales. This inversion of order is observed in the range of indebtedness $x$ between $10^{9}$ and $10^{10} \mathrm{JPY}$.

Comparisons for other origins categorised as intrinsic failure denote the same tendency of inversion. As indebtedness $x$ increases, the conditional probability of bankruptcy due to a link effect becomes larger than that of intrinsic failure. The same tendency is observed for different periods (See Figure 6.7), and in fact, the total number of bankruptcies due to origin (2a) in JFY 2001 was 1,731. Amongst those bankruptcies with indebtedness over 10 billion JPY, the number of those due to link effects amounted to 208 and that of those due to anemic sales only to 48 .

Figure 6.8 shows that there has been a change in the fraction of bankruptcies due to link effects over the past 10 years. Panel (a) charts the fraction of bankruptcies due to link effects, where the number of bankruptcies and amount of indebtedness are shown by open triangles and filled squares respectively. Panel (b) charts the dependence of bankruptcies due to link effects on the amount of indebtedness. The fraction of the number of bankruptcies due to link effects is about $10 \%$, and the fraction of indebtedness due to link effects is about $20 \%$. As the indebtedness increases, bankruptcy due to link effects also increases, and in particular the fraction due to indebtedness reaches $30 \%$, a fact indicating the importance of chain bankruptcy as a network effect.

\subsubsection{The Ripple Effect}

The question now arises as to what further effects are caused by the bankruptcy of company, for example, how many creditors are affected by the bankruptcy? 

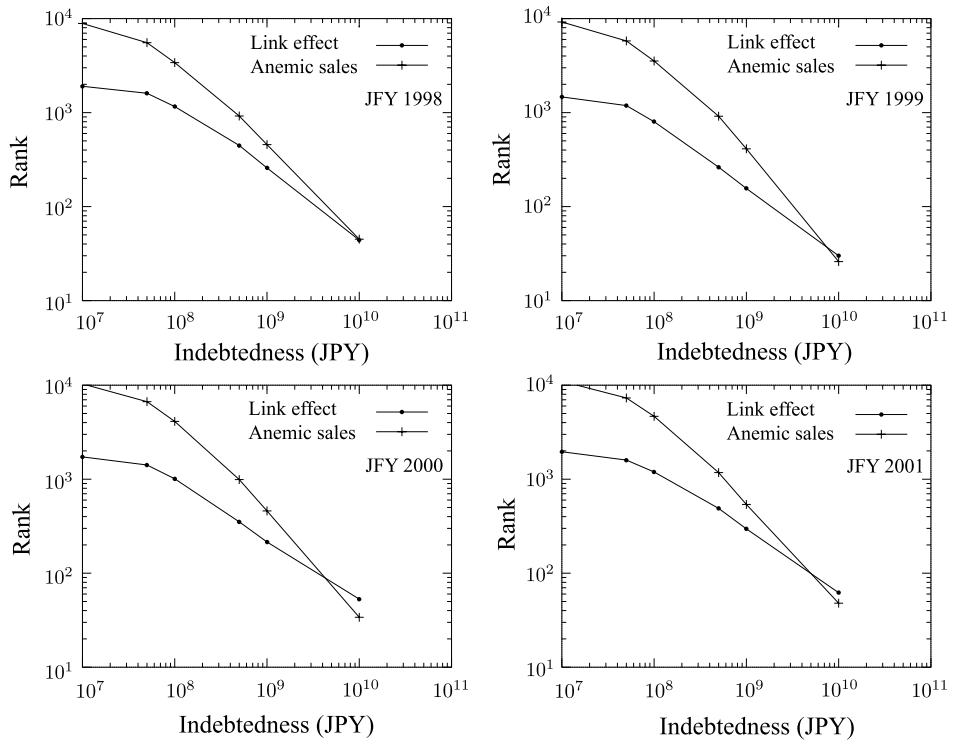

Figure 6.7: Rank-size plots of indebtedness at bankruptcy plotted for comparison between two major causes of bankruptcy (Annual reports on business bankruptcy, published by SMRJ) 

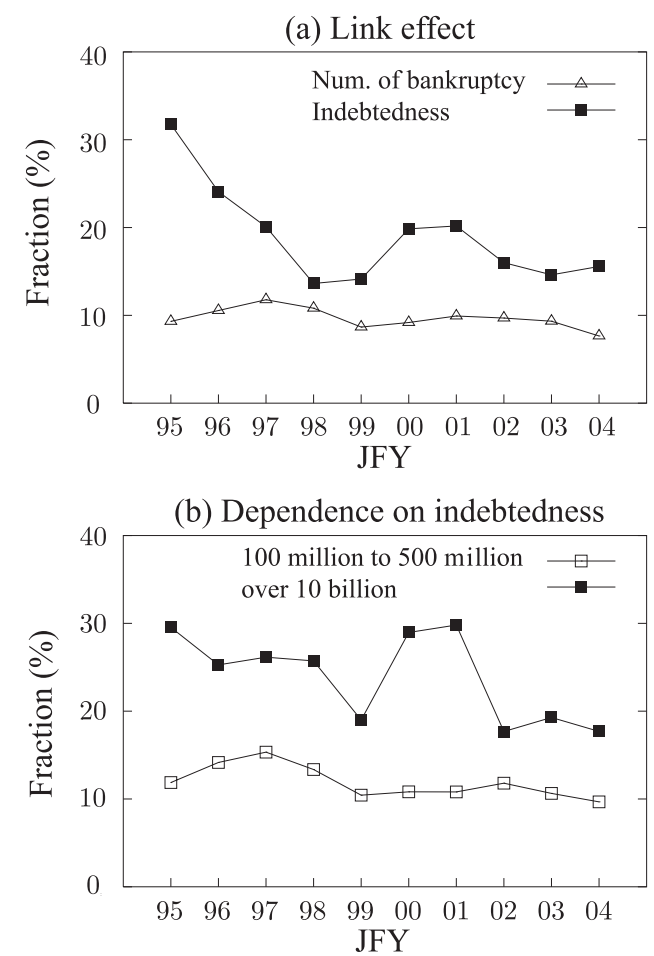

Figure 6.8: (a) Fraction of bankruptcies due to link effects, (b) Dependence of bankruptcies due to link effects on the amount of indebtedness 
As explained in the section on transaction networks, bankruptcy will obviously have consequences for workers and banks as well as production, since the propagation paths are not restricted to links relating to production but also to employment and finance links. In particular, any direct influence on banks would have a very large effect in terms of the quantity of debt and credit.

In pursuit of answers to these questions we will study an actual case of bankruptcy in detail (See Table 6.2), in fact a medium size chain retailer that became bankrupt during the year 2005 in Japan. The transactions in Table 6.2 can be summarised thus (The numerical number in parentheses reflects the number of cases): accounts receivable (65), leasing receivables (12), deposits received on sale (11), expense (7), cautionary obligation (1). Breaking out the data from the financing table we find financial institutes (8), other financial institutes (2), debt securities (2).

The indebtedness was about 4.7 billion JPY, of which 3.4 billion JPY was debt from twelve financial institutions, major commercial and regional banks. Table 6.2 shows that although a large fraction of the indebtedness was debt from financial institutions, the creditors affected by the bankruptcy were mainly companies engaged in goods transactions, especially vendors for the bankrupt retailer. 65 companies amongst 96 transaction goods creditors were creditors of accounts receivable.

Table 6.2: Number of creditors and amount of indebtedness for a bankrupt company

\begin{tabular}{|r|r|r|}
\hline Relationship & Number of creditors & Indebtedness $\left(10^{3} \mathrm{JPY}\right)$ \\
\hline \hline Transaction & 96 & $1,360,592$ \\
Financing & 12 & $3,393,976$ \\
\hline Total & 108 & $4,754,568$ \\
\hline
\end{tabular}

As explained before, a link in a transaction network corresponds to a relation between a debtor (borrower) and a creditor (lender). The effect of a bankruptcy propagates in the upstream direction in a transaction network, because that is where creditors are located. The impact of the propagation depends on the companies affected. For example, a large company would not be seriously disturbed by the loss of one customer and the inability to collect a single account receivable. However, a small company or a company whose major customer is the bankrupt company might be fatally affected. Note that this propagation process is different from the stochastic fluctuations described in the section 5.3, which are likely to be jump processes.

If the seriously affected company itself goes bankrupt, this is part of the process of the chain bankruptcy, and it should be noted that there are many companies upstream of a bankrupt company in such a chain. The workers and banks involved with the company are affected through the employment and financing networks. In the event of a bankruptcy the banks must increase the risk premium on the interest rate, thus exposing many other debtors to 
credit risk. This effect may even cause a credit crunch affecting many other companies further off in the financial network, a matter explained above in the section 5.3.

In fact, since almost any company can be affected by a chain bankruptcy, through the transaction, employment, and financial networks, it is essential to grasp the structure of the transaction network for effective risk management. In particular, the most important information concerns the financial condition of the customers of a company's customers.

There are about 100 companies upstream of the bankrupt company in the case considered above. We might ask whether this number is exceptionally large, and to obtain an answer to that question we will examine the degree distribution explained above in section 4.5.2. The fact that the degree distribution has a fat tail may yield interesting results about the number of a company's customers' customers.

When company A sells goods to the company B, we can postulate a directed link from A to B. Figure 6.9 gives the cumulative distributions of (a) in-degree (vendors) and (b) out-degree (customers) for the directed network consisting of thirty thousand companies, including about three thousand listed companies, in the JFY 2003. ${ }^{2}$ Both the in-degree and out-degree cumulative distributions have fat tails where the cumulative distribution $P_{>}(k)$ of degree $k$ shows the power-law behaviour $P_{>}(k) \propto k^{-\nu}$, and the values of $\nu$ are in the range of 1.4 to 1.5 for both in-degree and out-degree.

As we can see, there are many companies only one link upstream, due to the fat-tail nature of the degree distribution. The number of companies two links away upstream (that is, the friend-of-a-friend, as it were) is larger than might be expected in a rough estimation. If we assume that the average in-degree is 10 , then the question is how many vendors are there for the company's vendors. We can estimate this simply by ignoring the possibility of double counting, which would increase the number, and thus calculating $10 \times 10=100$.

However, a rigorous estimation shows that in fact the number of companies is much larger than this, because the fat tail of the degree distribution makes the probability of large degree companies finite.

For clarity, let us assume that all links in the network are non-directed, that is we assume a non-directed graph. The average number of companies upstream by two links, $n_{2}$, can be estimated by

$$
n_{2}=\left\langle k^{2}\right\rangle-\langle k\rangle
$$

as explained on p. 119. Here $k$ is the degree and $\langle\cdot\rangle$ is an average over the degree distribution. The average number of vendors is $\langle k\rangle$. Our rigorous estimation of the number of companies $n_{2}$ is thus much larger than the simple estimation $\langle k\rangle^{2}$, because the first term of the r.h.s. of the above equation becomes large for a fat tail distribution.

\footnotetext{
${ }^{2}$ Link data is not available for any listed companies, and it is presumed that distributions of sections with large degree are not affected by the lack of data.
} 
(a)
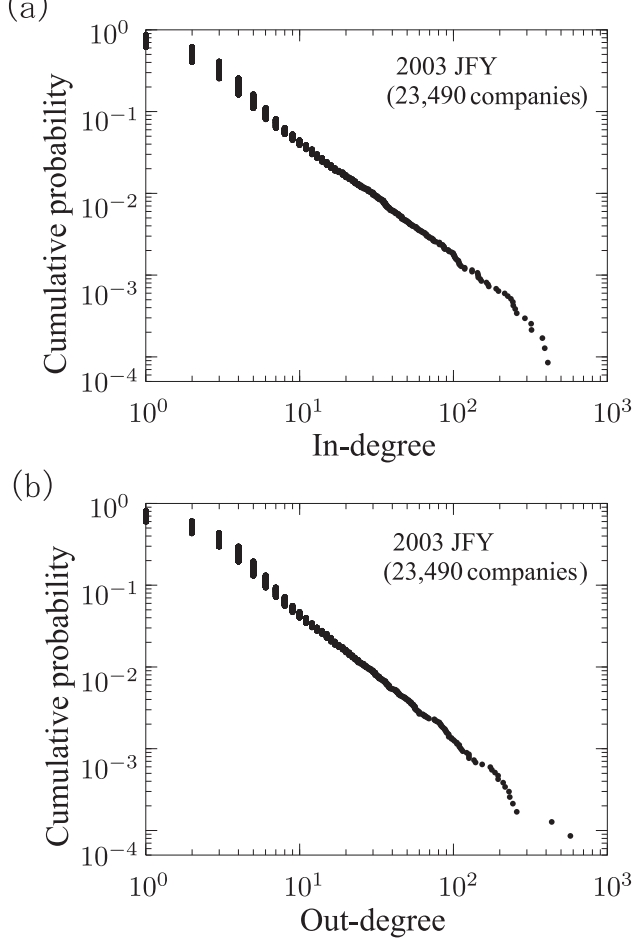

Figure 6.9: Cumulative distributions of (a) in-degree (vendors) and (b) out-degree (customers). 
For the transaction network, where the total number of nodes is 20,601, and the total number of links is 66,623 , we obtain

$$
\langle k\rangle=6.47, \quad\left\langle k^{2}\right\rangle=287 .
$$

With these numbers, the simple estimation leads to $\langle k\rangle^{2} \simeq 42$. By contrast, the rigorous estimation using Eq. (6.10) results in 281. The actual observation is $n_{2}=220$, which is very close to the estimate. ${ }^{3}$ See also Fujiwara and Aoyama (2008).

In summary, there are many companies upstream by one link, and the number of companies upstream by two links is much larger than might be imagined from a simple estimation, due to the fat tail of the degree distribution. At present, only companies upstream by one link are considered in credit exposure management, but this underestimates the risk, an error which we suggest could be corrected by employing the theory explained here.

As noted before, the impact of the propagation of credit risk depends on the character of the affected companies, for example a small company or a company whose major customer is the bankrupt company could well be affected fatally. It is possible to detect these weak paths in the transaction network in advance by examining the relationships from the downstream to the upstream side.

On the other hand, a large company is not affected seriously by an uncollectible account receivable or loss of a single customer, for the simple reason that a large company has many alternative customers. If the network is studied from the upstream to the downstream, a different view emerges. There are a great number of companies downstream by one link, two links, and three links from a large company, and since there are many weak paths in this set of links the possibility of a ripple from those companies at the downstream cannot be ignored. ${ }^{4}$

In this way even a large company that is thought unlikely to be affected seriously if the company loses a single customer could not ignore the impact of chain bankruptcy.

Figure 6.10 is a part of the transaction network consisting of a bankrupt company, creditors, and creditors of creditors, drawn for transaction data in JFY 2005. Nodes on the inner circle are creditors and nodes on the outer circle are vendors of the creditors.

If we want to be able to issue an alert of the likelihood of chain bankruptcy we need to detect weak paths in the transaction network in advance, and traditional credit exposure management is not sufficient for this purpose. Instead,

\footnotetext{
${ }^{3}$ Error in the rigorous estimate arises from two sources. One is that company $\mathrm{C}$ is one of the linked companies of the company $\mathrm{B}$ which are linked by the company $\mathrm{A}$, and company $\mathrm{C}$ is directly linked to the company $\mathrm{A}$. This means there is a triangle $\triangle \mathrm{ABC}$ and the cluster coefficient is not equal to zero. The other reason is that there is a quadrangle where $\mathrm{A} \rightarrow \mathrm{B} \rightarrow \mathrm{C}, \mathrm{A} \rightarrow \mathrm{D} \rightarrow \mathrm{C}$, and no link between $\mathrm{B}$ and $\mathrm{D}$.

${ }^{4}$ The ripple effect is a general term to indicate the effect of an influence from a secondary factor, a tertiary factor, and etc.
} 


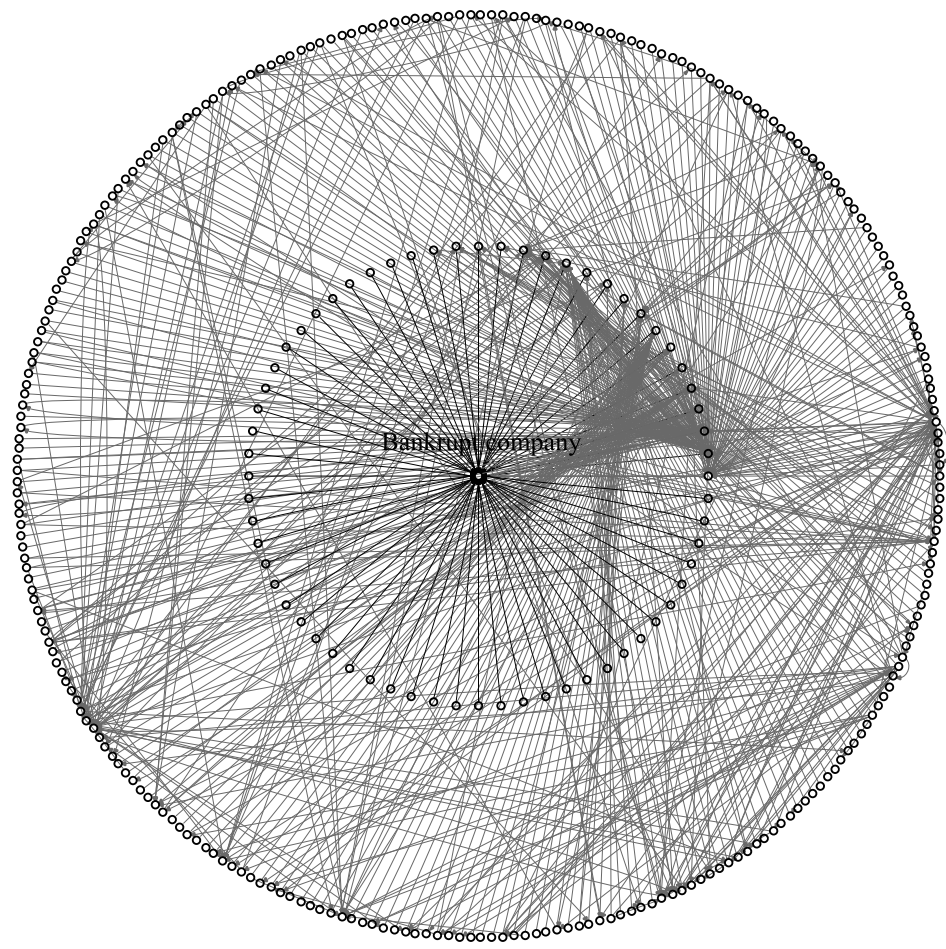

Figure 6.10: A part of the transaction network consisting of a bankrupt company, creditors, and creditors of creditors 


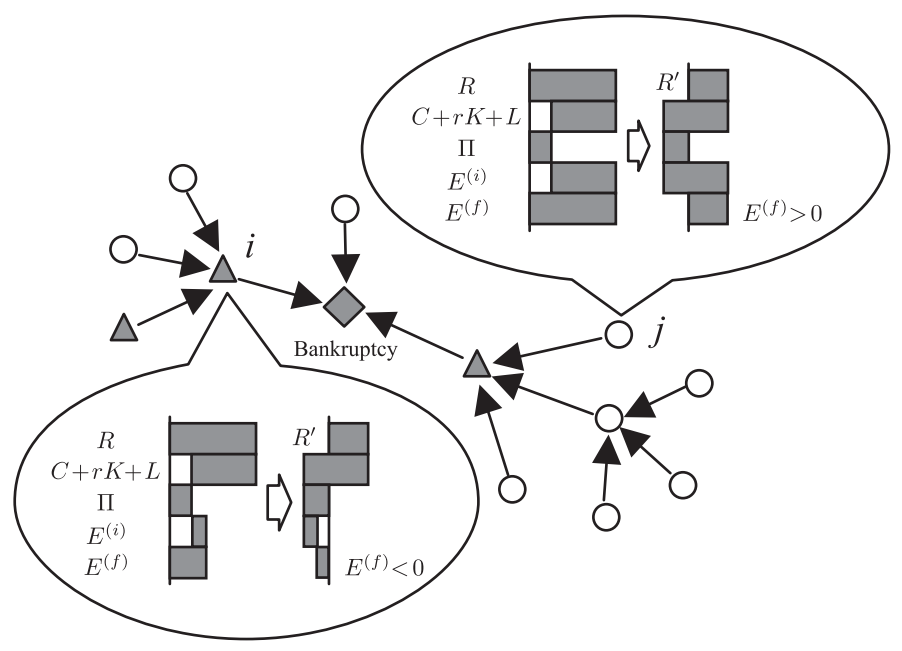

Figure 6.11: Model of chain bankruptcy in a transaction network

it is essential to capture the structure of the transaction network, and collaborative work engaging both the business and academic worlds needs to start immediately.

\subsubsection{Propagation of Credit Risk on the Transaction Net- work}

We will now explain another practical application of the network model explained in section 5.5, namely the simulation of chain bankruptcy in attempts to grasp the importance of accounts receivable, accounts payable, and capital structure.

The concept of chain bankruptcy in the network model is schematically shown in Figure 6.11. An arrow in the figure indicates the direction of the distribution of goods in the transaction network, thus the company shown by a rhombus is located at the outfall of the transaction network in the range of this figure. Note that such a company distributes goods to a company located further downstream, which is not shown in this figure. We assume that each company manufactured goods and then distributed them to a downstream company, and is waiting to call in accounts receivable. If the company shown by a rhombus is bankrupted by an unexpected accident, then this company cannot pay these accounts to upstream companies. The company $i$ shown by a triangle located at the left of the company shown by a rhombus expects to obtain profit $\Pi_{i}$ from sales revenue $R_{i}$ less total cost $C_{i}+r_{i} K_{i}+L_{i}$, and 


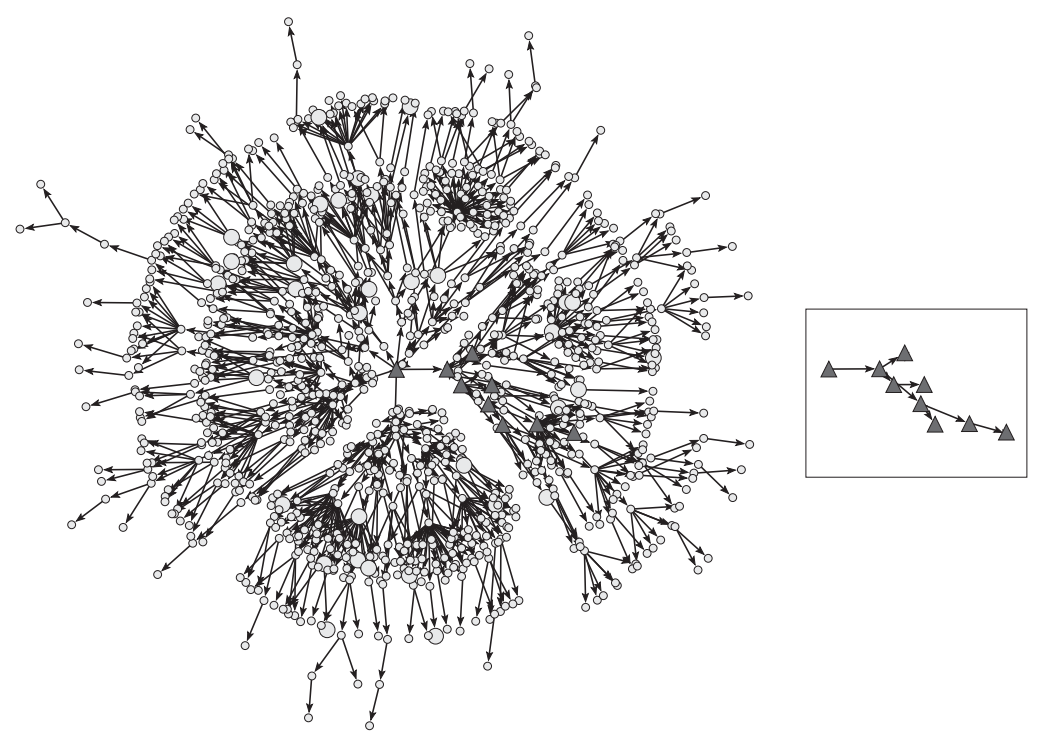

Figure 6.12: Results of the chain bankruptcy simulation

increases equity $E_{i}^{(i)}$ at the beginning of term to equity $E_{i}^{(f)}$ at the end of term. Because the company shown by a triangle cannot obtain accounts receivable, the sales revenue decreases from $R_{i}$ to $R_{i}^{\prime}$, that is to say it goes into the red. The company makes up for the loss by withdrawing part of its equity, and as a result the equity $E_{i}^{(f)}$ at the end of term becomes negative, and of course capital deficit is one of the definitions of bankruptcy. The bankrupt company cannot pay accounts payable, and so some upstream companies may become bankrupt, if they do not have sufficient equity to handle the loss of profit. We call this phenomena the propagation of credit risk through the transaction network. On the other hand, the company $j$ shown by a open circle located at the upper left of the figure has enough equity $E_{j}^{(i)}$ at the beginning of the term. This company retains this equity $E_{j}^{(f)}$ at the end of the term, even if the company makes up for the loss by withdrawing part of equity $E_{j}^{(i)}$ at the beginning of the term. In this case, the propagation of credit risk on the transaction network is terminated at the company $j$, because it has sufficient equity to absorb the shock.

We will now put the concept sketched above into a practical simulation and examine the propagation of credit risk. The transaction network used in the real network simulation consists of about 1,400 listed companies on the Tokyo stock exchange. It should, however, be noted that the initial values for sales revenue $R_{i}$, cost $C_{i}$, equity $E_{i}$, capital $K_{i}$, labour $L_{i}$, and interaction param- 
eters $k_{i j}$ were given virtual values. A company was added to the transaction network and three companies were arbitrarily chosen as suppliers for the additional company. Then we supposed that the additional company became bankrupt as the exogenous trigger of chain bankruptcy. The results obtained from this simulation are shown in Figure 6.12. The network is drawn from the company depicted by a rhombus at the outfall of the transaction network toward the linked companies at the upstream region of the distribution of goods. The companies which became bankrupt due to the propagation of credit risk are marked by triangles. The basic characteristics of chain bankruptcy are clearly visible, namely a chain of bankrupt companies stretching from the outfall to the upstream of the transaction network. The companies which terminate the bankruptcy chain are those with a small number of accounts receivable from the bankrupt company or those with sufficiently large capital $E_{i}^{(i)}$ at the beginning of term.

While the simulation explained in this section remains at a qualitative level, it is however possible to simulate chain bankruptcy quantitatively if the model parameters are set with actual financial statement data. By selecting an exogenously bankrupt company systematically, a quantitative simulation makes it possible to find weak credit risk links on the real transaction network.

\subsection{Business Model and Business Information}

In Japan there is a kind of industrial group known as a "Keiretsu", which is conventionally understood as an industrial group consisting of a family of companies linked to each other by share holding. In a narrow sense, the Keiretsu is a family of companies which are members of a consolidated accounting set. Recently, it has been pointed that industrial groups with low profitability need to re-activate their business by changing their fundamental business model through M\&A and the reconstruction of their portfolio of activities. In the last decade the Japanese electric machinery sector has used M\&A to initiate this sort of reorganisation, and recent cases are shown in Table 6.3. While it is intuitively obvious that one industrial group could record good results at the same time that another group may be having a very tough time in market, it is however difficult to judge their relative success simply by sampling performance at a particular period.

In this section, we will first explain the basic advantages and drawbacks for an industrial group, and then explain how the ability to respond to a sudden accident is a very important advantage. Subsequently, we will verify the business synergy expected in industrial group, and finally we propose to combine business information and computer simulations in order to work out an innovatory business model. 
Table 6.3: Cases of M\&A in Japanese electronic component industry

\begin{tabular}{|c|c|c|}
\hline Year & Merging company & Merged company \\
\hline \hline 1999 & Hitachi Plasma Display & $\begin{array}{c}\text { PDP divisions of } \\
\text { Hitachi and Fujitsu }\end{array}$ \\
\hline 1999 & Elpida Memory & $\begin{array}{c}\text { DRAM divisions of } \\
\text { Hitachi and NEC }\end{array}$ \\
\hline 2001 & Matsushita Electronics \\
\hline 2001 & Sony Ericsson Mobile Communications & $\begin{array}{c}\text { Cell-phone divisions of } \\
\text { Sony and Ericsson }\end{array}$ \\
\hline 2002 & Panasonic & $\begin{array}{c}\text { Matsushita Tushin-Kogyo, } \\
\text { Kyushu Matsushita, } \\
\text { Matsushita Seiko, } \\
\text { Matsushita Kotobuki-denshi, } \\
\text { Matsushita Densou system }\end{array}$ \\
\hline 2003 & Hitachi Global Storage Technologies & $\begin{array}{c}\text { HDD divisions } \\
\text { of Hitachi and IBM }\end{array}$ \\
\hline 2003 & Renesas Technology & $\begin{array}{c}\text { System LSI divisions of } \\
\text { Hitachi and Mitsubishi Electric }\end{array}$ \\
\hline 2003 & PIONEER & LCD division of NEC \\
\hline 2004 & S-LCD & Sony and Samsung Electronics \\
\hline 2005 & Sharp & LCD division of Fujitsu \\
\hline
\end{tabular}




\subsubsection{The Industrial Group as a Business Model}

A business model is a scheme used by a company to define how it intends to earn profits in a selected industry sector. In other words, the business model defines the origins of profit, the flows of goods and money, the relationship between customers and vendors, and the field of business, and it forms the basis of the business strategy.

Some readers might be familiar with the word "business model" from the term "business model patent", which is a patent relating to a business method

\section{The world car platform of General Motors Corpo- ration}

Most cars are designed, manufactured, and sold in order to fit the needs of a specific market, such as the US. Only a few compact cars and a few luxury cars are manufactured in one country and then exported worldwide.

In 1970s, General Motors Corporation in USA invented an outstanding business model where cars to fit the various market needs of many countries were designed and manufactured with minor modifications on a versatile platform. This business model was called the world car platform, and it formed part of General Motors' strategy to dominate the world market.

Starting with the T-car in 1974, General Motors released its X-car in 1979, and then its J-car in 1981. However, their plan to achieve global market supremacy resulted in failure, partly due to major drawbacks in the business model itself (Piore and Sabel, 1984). The first drawback was the distribution of manufacturing needed in emerging countries. Low-cost and stable labour forces in emerging countries proved to be difficult to obtain, and General Motors suffered from serious problems, such as demand for higher wages and various labour disputes immediately after starting to manufacture cars at the newly constructed factories. The second drawback was concerned with global component procurement. This method of component procurement requires holding a very large parts inventory in a car manufacturing factory. If faulty parts were delivered from very distant distributed component factories, the company's managers and production engineers could not promptly replace them. This situation was in marked contrast to the Just-In-Time manufacturing system invented by the Toyota Motor Corporation in Japan.

It is important to note that the World Car strategy failed for reasons other than product quality. We know this at first hand, since one of the authors (Y.I.) once owned a used J-car, a Pontiac J2000, in the US in the late 1980s. Although the J-car was never a car to attract enthusiasts, it was a workhorse without serious problems. 


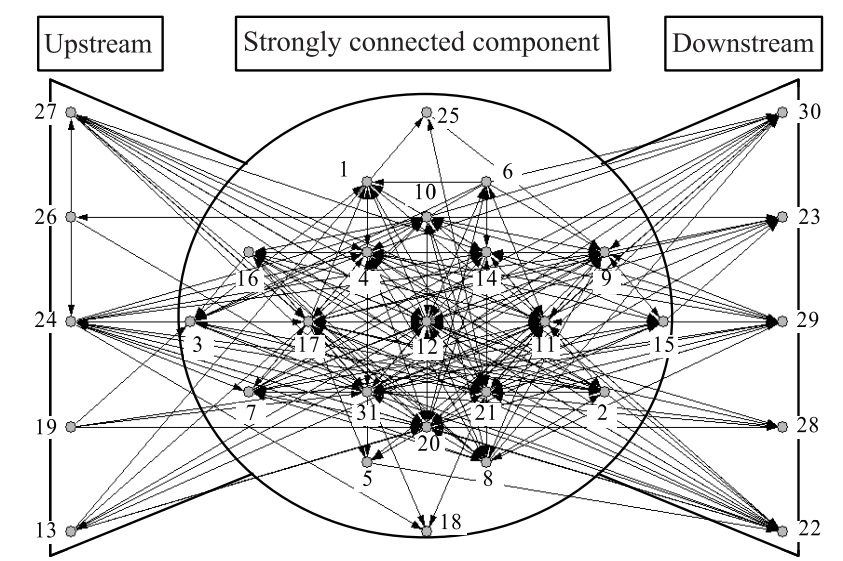

\begin{tabular}{|c|c||c|c|}
\hline Number & Industry Sector & Number & Industry Sector \\
\hline \hline 1 & Food & 2 & Textiles \\
\hline 3 & Paper Manufacturing & 4 & Chemicals \\
\hline 5 & Pharmaceutical Manufacturing & 6 & Oil \\
\hline 7 & Rubber & 8 & Ceramics \\
\hline 9 & Steel & 10 & Non-ferrous Metal \\
\hline 11 & Machinery & 12 & Electric Machinery \\
\hline 13 & Shipbuilding & 14 & Automobile \\
\hline 15 & Transportation Equipment & 16 & Precision Machinery \\
\hline 17 & Other Manufactures & 18 & Fishery \\
\hline 19 & Mining & 20 & Construction \\
\hline 21 & Wholesale & 22 & Retail \\
\hline 23 & Railroad & 24 & Land Transportation \\
\hline 25 & Marine Transportation & 26 & Air Transportation \\
\hline 27 & Warehouse & 28 & Telecommunication \\
\hline 29 & Electric Power & 30 & Gas \\
\hline 31 & Service & & \\
\hline
\end{tabular}

Figure 6.13: Coarse-grained transaction network

using information technology, such as computers and the internet. The business model patent has an infamous reputation for being used to patent the application of business method even though it is not recognised as an invention. Concerns about the validity of the business model patent do not affect the fact that there is no doubt that the business model is essential for well performed business.

Figure 6.13 is a coarse grained transaction network in Japan at the industry sector level of resolution (Ikeda et al., 2008). Arrows in the figure indicate the flow of goods, and the numbers indicate industry sectors as shown in the table. Industry sectors related to infrastructure and raw material, such as air transportation, land transportation, warehousing, and mining industries, are located in the upstream zone. On the other hand, industry sectors subject to 
consumers, such as gas, railroad, electricity, telecommunication, and retailing industries, are located in the downstream zone. In the central part of the network, which is a so-called "strongly connected component", we find the electric machinery, automobile, chemical, service, and wholesale industries.

In the electric machinery and automobile industries located in the centre of the coarse grained transaction network, there are several very large industrial groups employing both vertical integration and a horizontal division of work. Why and how are these industrial groups formed? Let us consider the business model of an industrial group.

By examining their corporate histories we find that many industrial groups were formed as a result of mergers and acquisitions (M\&A) and the split-up of companies, actions which are considered to have the following advantages.

- Management with a long-range perspective: When a company is protected by mutual shareholding, the company is free from the threat of M\&A by competitors or investment funds, even if the stock price becomes low. Thus, the managers of the company could take the advantage of that security and run the company with a long-range perspective without worrying about imminent M\&A drive by competitors or investment funds. In addition, managers could maintain a wage system policy grounded in seniority and guaranteed lifetime employment.

- Augmentation of customers and agility: If a company is split up, a subsidiary company could start to sell goods to competitors of the parent company, and as a result the subsidiary company can enlarge its customer base. In addition, small companies are often more agile in decision and action than larger competitors, and subsidiary companies are small in general. Thus in the industry sector, where agility is requisite for high performance, it is sometimes regarded as advantageous to split a company to reduce overall size.

- Ensuring job security and adjustment of cost structure: By relocating personnel from the parent company to important posts in the subsidiary company it is possible to run that company with a management policy consistent with that of the parent. Furthermore, creating new posts in the subsidiary company assists in maintaining a wage system grounded in seniority and guaranteed lifetime employment. In addition, managers save labour costs for the whole industrial group by holding down the wages of the subsidiary companies to fit their profit level. Furthermore, the subsidiary company can maintain friendly relationships with the parent company through straightforward personal acquaintance.

- Reduction of transaction costs: The parent company does not need to incur costs when seeking credible companies for outsourcing or other transactions. Savings are also expected on R\&D costs because of the collaboration between the parent and the subsidiary companies, and 
flexible transactions grounded in stable relationships. Moreover, the subsidiary companies can reduce their operating expenses in finding new customers and understanding their needs because of the parent company's broad base of information. Surprisingly, before consolidated accounting was introduced in Japan, subsidiary companies were forced to buy goods from their parent company to reduce the stockpile and increase the revenue of the parent company, and this was almost certainly one of the historical reasons for the success of the industrial group.

- Coping with credit risk: It is possible to transact business between members of an industrial group without incurring credit risk. Even if an unforeseeable accident occurs, related companies are expected to make spontaneous arrangements to deal with the emergency without previous agreement, since companies belonging to the same industrial group are strongly tied by common interests. If the transaction is a one-shot sale, arrangements to deal with the emergency must have been designed previously, and unless the arrangements are strangely far-sighted and well designed, it is not likely that they will deal effectively with the emergency.

However, recent changes in the business environment are showing that industrial groups have some drawbacks. As reform of corporate governance rises up the agenda, there is a tendency to attach more importance to short-term profitability in the interests of shareholders. For example, indices of profitability, such as the return on equity (ROE), have been introduced for companies, and capital adequacy requirements, such as the bank for international settlements (BIS), have been introduced for banks. As a result industrial groups based on mutual shareholding are steadily being dismantled.

Transactions between companies in the same industrial group are stable and cost-saving because credit administration is not required. On the other hand, inefficiency due to a cozy and uncritical relationship gives cause for concern. During Japan's high economic growth period in 1960s, if there was inefficient transaction in part, most companies could obtain enough profit because of economic growth, but in today's business environment we could not find a leading company willing to predict the direction of general economic movement. Consequently, it is increasingly said that doing business with various companies on a flexible basis is more efficient and profitable than doing so statically with related companies in an industrial group.

The drawbacks of the industrial group are apparent even for R\&D when seen as an origin of future profit. Let us consider games as an analogy for business. Victory or defeat in chess is determined largely by the ability to consider an opponent's moves. On the other hand, in Poker a player has to play cards by considering their own cards in the context of restricted information about their opponents' cards. This distinction resembles that between a traditional business and a new post-internet business.

For example, in a traditional business, such as the electric machinery industry sector, a small number of large companies conduct basic-study at their 
own corporate laboratories and apply the research output to their business. The process by which an industrial group moves from R\&D to commercialisation of products is called closed innovation, or the linear model of product development.

Meanwhile, the product cycles of software products and information equipment in the post-internet information society have become much shorter. A competitor might suddenly introduce a product which is competitive and based on a new and superior technology. Closed innovation does not work well in such a situation.

Consequently, a new method called open innovation (Chesbrough, 2006) is attracting attention. This is a method of product development where the boundary between companies is not clearly marked. If a company does not have technology required for the development of a new product, the company buys the technology from other companies. On the other hand, if a company decides not to use their own technology for the development of a new product, the company sells the technology to another company or to a split-off venture business.

There are a several points to keep in mind when initiating open innovation. Firstly, it is important to recognise that there is no intrinsic economic value to technology; a technology can only be evaluated in the context of the business model of a company. Thus, it is necessary to determine the sale price of technology in the light of the company's own business model. Note, however, that open innovation does not mean that R\&D at a company's own laboratory is unnecessary, since they must take on the difficult tasks of defining systems to integrate the technologies. Furthermore, open innovation is not necessarily a panacea for all new business, and it is extremely important to evaluate which enterprise is suitable for the open innovation and which is not. For example, although the effectiveness of open innovation for software development has been proved, in-depth consideration might be required before applying it to hardware development consisting of various complex components.

\subsubsection{Robustness of Industrial Group}

One of the outstanding features of the company network for the horizontal division of work is the ability to respond to unexpected accidents. ${ }^{5}$ In a business environment with high uncertainty the superiority of the horizontal division of work is becoming apparent when compared with vertical integration. Whereas vertical integration is characterised by the division of labour in each layer of the business process, horizontal division possess a versatile but specialised strength called flexible specialisation.

A company seldom obeys an order from another company in the horizontal division network. Instead, each company exhibits a sort of vague willingness to behave according to the expected demand from other companies, and a tendency to correct that expectation in the light of interactions with other

\footnotetext{
${ }^{5}$ This section is based on the chapter 9 of Watts (2003).
} 
companies in the network. By considering the effect of vagueness, the horizontal division network can be understood as a hierarchical network of information-processing nodes.

When load is centralised on a certain node, and consequently the processing speed of the node is decreased, homogenisation of load will result if a bypass link is added to this node. The network generated by adding such bypass links is called a multiscale network. The multiscale network possesses robustness, which means that the whole network does not break down as the result of a single accident, such as destruction of production system due to fire or the bankruptcy of large company. This robustness is an outstanding feature of the company network, and is not intended or designed by managers.

This feature is dramatically in evidence in the recovery after a serious fire at the Aisin Seiki Co.'s Factory No. 1 in Kariya on February 1, 1997. Aisin Seiki Co. was the exclusive supplier of the p-valve, a part of the break system used in almost all Toyota's cars. Production was concentrated at the Aisin Seiki Co.'s Factory No. 1, which manufactured more than 30 thousand sets of p-valves per a day. Since Toyota manufactures 15 thousand cars per a day, stocks of the p-valve would be consumed within two days. The lack of this tiny part caused the stoppage of the whole production process in Toyota, an astonishing fact.

Even worse, the fire not only stopped the supply of the p-valve but also destroyed the production system specialised for the p-valve. Toyota and Aisin were worried that several months would be required for recovery, a matter of great economic significance even for Toyota. However, the production of the p-valve was restarted on February 4, 1997, just three days after the fire, as the result of the cooperative action of more 200 companies in the industrial group, and all this without any support from Toyota itself. Toyota restarted car manufacture and daily production was close to 14 thousand on February 10, 1997. It is believed that the key to this dramatic recovery was the robustness of the multiscale network in the Toyota industrial group. Disaster recovery, then, is a by-product of the network constructed during a normal operation.

From this impressive story we learn that the manager of the flagship conglomerate leading an industrial group should not force business plans on to subsidiary companies, but rather should encourage decision-making in business by exploration of the vaguer aspects of the company network. We believe that a robust industrial group will result from this decision-making strategy.

\subsubsection{Synergy in Industrial Groups}

It is widely believed that synergy for profit is to be expected in an industrial group. To put this in concrete terms, such synergy means that a company acquiring another company satisfies a certain condition and so makes a larger profit than the sum of profits for each company, in keeping with the definition of synergy which describes it as a phenomenon where the total value of the linked components is larger than the sum of values of individual components. On superficial examination it might be thought that when the company A 
selling the product $x$ and the company B selling the product $y$ are merged, the sales revenue is increased by selling both products $x$ and $y$ through the same sales person. In addition the merged company might obtain larger profits with the same sales revenue by reducing various costs, e.g. management costs in headquarters. Synergy seems attractive. But is any of this really true?

We can test the hypothesis that the increase of profit is larger than the increase of company size by postulating an industrial group. The company invests the labour $L$ and the capital $K$ and obtains the value added $Y$ by production activity. The labour $L$ and the capital $K$ are the inputs and the value added $Y$ is the output. In general, multiplying the inputs $L$ and $K$ by a factor of $\lambda$ increases the output $Y$ by $\kappa$ times;

$$
\kappa Y=F(\lambda L, \lambda K)
$$

Here the function $F(\cdot)$ is a production function explained in the section 3.5.1. If $\kappa=\lambda$, the size of labour $L$ and capital $K$ is increased by a factor of $\lambda$ and accordingly the value added $Y$ is increased by the same factor. This means that there is no synergy at all.

The labour $L$, the capital $K$, and the value added $Y$ are calculated using financial statement data for each company belonging to an industrial group. The labour $L$ is the sum of the labour cost, the employment cost, and the welfare expenses. The capital $K$ is the sum of the tangible fixed asset and the allowance for depreciation. The value added $Y$ is the sales revenue less the cost of raw materials and other expenses, such as electricity costs. In this way the number of points equal to the number of companies are obtained in the three dimensional space $(L, K, Y)$. Parameters $A, \alpha$, and $\beta$ of the production function

$$
F(L, K)=A K^{\alpha} L^{\beta}
$$

were estimated in order to reproduce the distribution of data points. Here it should be noted that the condition of "no synergy" is

$$
\alpha+\beta \leq 1
$$

because the relation

$$
F(\lambda L, \lambda K)=\lambda^{\alpha+\beta} F(L, K)
$$

holds.

Figure 6.14 shows the result of data analysis for twenty-four companies listed in the first section of the Tokyo Stock Exchange and belonging to a Japanese industrial group. The results of the parameter estimations are statistically significant. The average value in the past 20 years is

$$
\alpha+\beta=1.001 \pm 0.033 \text {. }
$$

Thus, there is no evidence of synergy. Indeed, the data suggests to us that the whole system of linked components is equal to the sum of individual 


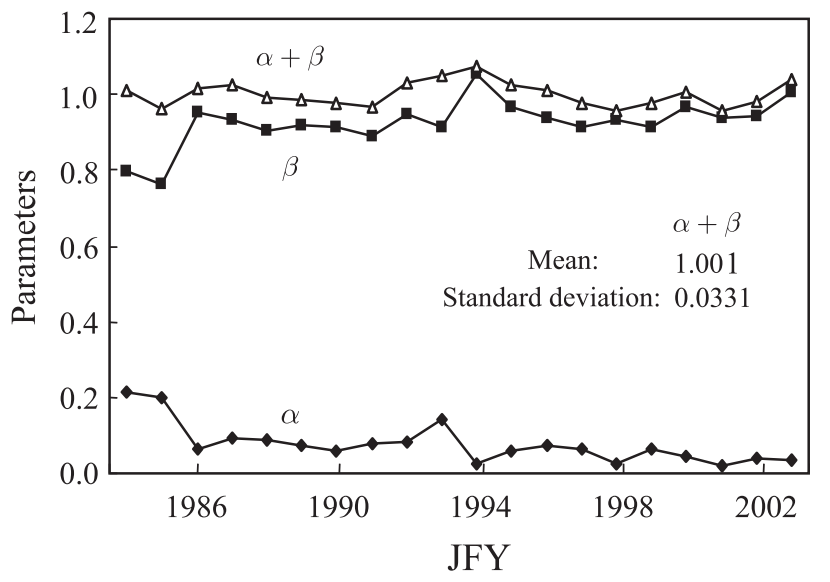

Figure 6.14: Parameters of the production function for companies belonging to a Japanese industrial group

components. Of course we cannot deny the existence of small scale synergy which is undetectable in an analysis using publically available data, but the result does mean that we cannot expect significant synergy for the existing business model or any extension of it, though it might be possible for us to produce drastically innovative business models which realise large scale synergy. Time will tell.

\subsubsection{Business Information Systems}

The previous section showed that we cannot expect an increase of profitability simply by increasing company size. It rather seems that the most important element in creating high profitability is the business model, and if larger company size is required to realise the business model, the use of M\&A might be a pattern for success. We might now ask, what is the key for the production of innovative of business models. This is a really hard question to answer, and there are many possible answers. However the first step necessary for innovation is common to all the answers, namely, that it is necessary to understand the situation of your own company in terms of the relationship between customers and vendors.

Quantitative analysis of the profitability of the business model, based on the newly clarified facts about the own company's business, is the deciding factor in predicting success and failure. In particular, complex network analysis and an agent simulation are invaluable tools for understanding the relationships and quantitative evaluation of profitability. 


\section{The Application of Business Information}

Various tasks are conducted in each division of a company, and these tasks are categorised as routine or non-routine. A routine task is an operation repeated regularly according to predetermined procedures. On the other hand, a non-routine task is an operation which is different on each occasion, occurs irregularly, and for which only a rough procedure is given. Business information systems are categorised in the same manner. Conducting routine tasks is associated with the mission-critical system, that is to say the system that processes the information of the company as a whole for material procurement, physical distribution, sales, finance, and personnel affairs. The system is characterised by information transactions concerning the relationship between customers and suppliers. On the other hand, the handling of non-routine tasks is associated with business information systems and related software. The business information system processes information for tasks relating to each individual division, and the system is characterised by the linking of many software packages. Of these tasks, decision-making in the business strategy is the most important target of the business information system.

There is a long history to company information processing, and many systems were developed in the era of the mainframe computer. Many missioncritical systems are being reconstructed in order to facilitate the interactive transaction of many core corporate functions. It is now becoming common to process information for material procurement, physical distribution, sales, finance, and personnel affairs in an integrated manner using an enterprise resource planning (ERP) system. In addition, it is also coming to be normal to use supply chain management (SCM) in the management of a series of task chains from procurement of materials and components to production, physical distribution, and sales in order to improve efficiency relating to delivery date and inventory management. The SCM is categorised either as an intra-company SCM, which manages material procurement, physical distribution, sales, and finance within the same company, or an inter-company SCM, which manages relations between materials companies, manufacturing companies, customers, wholesale companies, and retailers.

Initially, we will explain the noticeable advance of the mission-critical system from the perspective of innovation in the business model. Fortunately, electronic disclosure of financial statement data in eXtensible Business Reporting Language (XBRL) is now common (Bergeron, 2003). XBRL is a computer language suitable for the exchange of financial statement data, and is based on and similar in principle to the eXtensible Markup Language (XML).

In XML, flexible information exchange is made possible by tagging each piece of information. XML is a meta language, that is to say it defines a specific language, such as XBRL. Until very recently, the means for obtaining financial statement data was restricted to downloading data for an individual company or buying bulk data from a specialised data provision company. In 
addition, there were other problems such as the fact that financial data for listed companies were basically annual, while that for unlisted companies was incomplete. As the use of electronic disclosure employing XBRL becomes more widespread these problems are expected to be resolved.

The disclosure of financial statement data on a quarterly or a monthly basis, which is desired from the point of view of corporate governance, will be made possible by using the ERP system to hold and process data written in $\mathrm{XBRL}$. It is also anticipated that there will be a reduction in paperwork costs and increases in the speed of decision-making for business strategy formation. If disclosure on a monthly basis is widespread for small and medium-sized companies as well as large companies, the credit risk for customer companies will be captured in real time. When that occurs, automatic data acquisition using spidering programs, such as Googlebot, Scooter, and MSNbot, will become an effective tool for business. It is noted here that the most important target outcomes for the business information system and coupled software are increases in the rapidity of business strategy decision-making and real-time capturing of credit risk for customer companies.

We will now explain an electronic commerce system using XML. Electronic commerce is a business transaction using a computer network intended to reduce transaction costs and improve customer service. Electronic commerce transactions are categorised as business to business (B2B), business to consumer (B2C), or inter-consumer (C2C). In particular it is expected that the $\mathrm{B} 2 \mathrm{~B}$ transaction will lead to strengthen the above mentioned inter-company SCM.

Traditional electronic commerce using electronic data interchange (EDI) has been used only for large companies and their partner companies, because a dedicated program and a dedicated communication line are required. In contrast with the traditional electronic commerce, XML based electronic commerce uses the internet and this facilitates low cost and flexible transactions. Furthermore, the integration of business processes using XBRL might bring even higher efficiency, as is the case with the ERP system.

According to the ministry of economy, trade and industry in Japan, the market size of B2B electronic commerce in Japan was 1,939 billion USD in 2005, and the US Department of Commerce reports that the market size of the B2B electronic commerce in the USA was 1,821 billion USD in 2004 . Direct comparison of these two countries is difficult, because the timing of aggregation is different, and the Japanese statistics includes both transactions using the internet and dedicated communication lines. In fact, according to Global Industry Analysis Inc., the market size of electronic commerce in the Asia-Pacific basin was 1,700 billion USD in 2004 and 3,753 billion USD in 2005. Estimation of the market size of B2B electronic commerce in Japan in 2004 using the above data is $1,939 \times 1,700 / 3,753=0.878$ billion USD. This means that the market size in Japan is about half the size of that in the US.

Meanwhile, it is reasonable to expect the advance of business information systems and coupled software, reflecting the advance of the mission-critical system. An agent simulation based on data analysis of the transaction data 
of B2B electronic commerce, accompanied with the ERP system and the SCM system, could forecast demand of goods and supply of components simultaneously for many companies on the transaction network. This forecasting method could provide more reliable results than the conventional method, which uses only data for a specific company, and as a result benefits will be obtained in the management of business risks such as inventory growth and opportunity loss, the real-time capturing of credit risk for customer companies, and the acceleration of decision-making in the business strategy.

\section{The Application of Complex Network Analysis}

A very large quantity of data on inter-company transactions other than B2B electronic commerce is currently dormant in the files of commercial banks, since individual records of settlement operations for purchase and sales have to be recorded in the mission-critical system of the relevant banks. Consequently, the details of commercial distribution in the Japanese economy could be revealed by analysing the combined records of settlement operations together with the B2B electronic commerce data. If these data become available, important steps could be taken towards the design of a new high profitability business model.

As the Aisin Seiki case shows, the manager of a flagship conglomerate leading an industrial group should not force business plans on to subsidiary companies, but rather should encourage decision-making in business by exploring the vaguer aspects of the company network. But studying this requires data covering changing relationships on the transaction network, a matter we refer to as the reconnection of the transaction network. We anticipate that relationships in $\mathrm{B} 2 \mathrm{~B}$ electronic commerce are relatively flexible, and thus that many reconnection events are recorded in the transaction data, a fact that might open up the potential of studying the characteristics of reconnection, research which is currently impossible. If we were able to successfully model reconnection it is probable that we would observe that the information system itself makes decisions by exploring the vaguer aspects of the company network, thus opening the door for new methods of business risk management.

In the present complex network analysis, several network indices have been calculated for selected industry sectors. This is rather like studying the tree which happens to be in front of you, although the ultimate purpose of your research is to clarify the structure of the forest as a whole. Of course, a biased selection of subject matter is not an appropriate approach. It would be better to begin by calculating the various network indices for the whole network, and then selecting the subject of analysis on the basis of those obtained indices. To go back to our analogy, this would be like drawing a map of the forest at the outset, and then using that map to select which trees within the forest are to be studied. We have no doubt that by using large amounts of real-time data and sophisticated analytic methodologies it will be possible in the near future to capture the flows of money and goods in real time in order to detect economic problems at an early stage. 


\section{The Application of Agent Simulation}

The application of agent simulation is growing in importance in the study of innovative business models. In many research areas a PC cluster, which is a group of personal computers linked to each other through a high speed network to form a single high-speed virtual computer, is now being widely used. The processing speed is increased proportionally to the number of linked PCs, because the sequence of calculation is divided and is then performed in parallel. The fastest supercomputer in the world at present (June 2009) is the Roadrunner set up at the Los Alamos National Laboratory in the US. Roadrunner consists of 129,600 processors and performs at a computation speed of over 1 petaFLOPS. ${ }^{6}$ The fastest supercomputer in Japan at present is the Earth Simulator, which consists of 1,280 processors and performs with the peak speed of 0.122 petaFLOPS. The massively parallel type of supercomputer is not only much faster than the vector type with fewer processors, but also cheaper. For this reason, PC-based massively parallel supercomputers are becoming understandably popular.

In the decade ahead, the performance of computer and networks will improve still further, and it will be plausible to study new business models using agent simulations, including financial markets and consumers as well as companies and banks, depending on the problem to be solved. The aim is to study economic activity by simulating the whole economic network consisting of various agents, each behaving autonomously in order to obtain larger profit through the reconnection of the network.

If the business information system described in this section is realised, we will be able to obtain a company's data changes in real time rather than on a yearly basis, and it will therefore be possible to capture flows of money and goods in real time, which will probably have a very significant impact on practical business. This is a remarkable goal, and while there are many problems ahead, none of these are, in our view, insoluble. It is no exaggeration to say that we are at a turning point in applying the theoretical results of Econophysics to practical business situations. All that is needed is more hard work.

\footnotetext{
${ }^{6} 1$ petaFLOPS stands for 1 quadrillion floating-point arithmetical operations per second.
} 
246 CHAPTER 6. PERSPECTIVES FOR PRACTICAL APPLICATIONS 


\section{Epilogue}

Salv.: Greetings, Simplicio, Sagredo. You are both looking very thoughtful; which I hope is a good sign and shows that you have made good progress with Econophysics. In any case, I am very eager to hear what kind of impression the book has left upon you?

Simp.: Well, I thought the most interesting Coffee Break bit is ....

SAGR.: Oh heavens above; I sometimes wonder if you are a serious person, Simplicio. I haven't forgotten that it was you made our good friend Galileo go down on his knees before the Pope.

SimP.: That is a long time ago.

SAGR.: Indeed, and it is still fresh in my memory.

Salv.: Now, now, friends, let us keep our eye on the matter in hand. Sagredo, you first; tell me what you think.

SAGR.: Well, in spite of what you said about it being written as non-technically as possible and for a wide range of readers, I found much of the argument quite complicated. In any case, I learned of many concepts that were new to me, for example the Pareto distribution, the fat tail, Gibrat's law, complex networks, and open innovations, to name a few.

SALv.: Good. At least you are now free from the restrictive view of the Normal Gaussian distribution. Incidentally, our good friend Eugene Stanley has a very inspiring thing to say about the current global economic crisis in relation to the Pareto distribution (Stanley, 2009).

SIMP.: Which is..?

SALV.: He pointed out that there is a big difference in the way that physicists and traditional economists approach laws and the theory behind them. Our economist is, by and large, unwilling to accept a law if there is no complete theory behind it. But physicists, as Professor Stanley says, 'cannot afford this reluctance'. There is so much that we don't understand that even very useful laws, Newton's for example, or those of Coulomb, are discovered long before the theoretical underpinning exists. The same is probably true of economics. 
SAGR.: Oh, I see. Then he must be implying that economists cannot afford their reluctance now; they should overcome this false and unscientific conscience, and be a little lighter on their intellectual feet, as it were, particularly if these laws give you some degree of understanding that returns as power, as the English philosopher Coleridge once said.

SIMP.: Precisely; you see it immediately, and the value could be enormous.

SAGR.: Quite, if this scientific research could be applied to design of better, more resilient economic systems, those responsible would truly deserve the thanks of mankind.

Simp.: Forgive my pouring cold water on this little victory parade, but surely what I have seen described in this book is a long way from providing the panacea that you seem to envisage.

SAGR.: There is indeed more work to do, and the authors wouldn't claim anything else, but that is no reason for failing to see the potential. May I remind you, Simplicio, you refused to even look through Galileo's telescope, and if you don't mind me saying so I think you are trying to put your intellectual blindfold again.

SALV.: I would prefer not be so hard on Simplicio. Let us look back and try to persuade him of the rich possibilities here: What have we have learned about the networks made by companies and financial institutions?

SAGR.: That they are made of multiple layers; trades, stock-holding, multiple positions held by executive-officers, money-lending, joint patentapplications, to name a few aspects. Briefly, we have learned that these interlocking networks are almost unimaginably complex.

SALv.: Precisely, and our conclusion must be that the behavior of such economic agents should be modeled and understood on the basis of the interactions of the complex networks of which they are part.

Simp.: Easily said, Salviati, but how do you propose to handle and model such a complex system?

SALV.: It is data-intensive, but simulation is one option: for example problems in cosmology, such as the collision of galaxies, are modelled through the calculations of very large parallel computers. The same is true in the microscopic world, where quantum chromodynamics placed on a spacetime lattice are beginning to replicate a realistic spectrum of elementary particles.

SAGR.: What a thought, an Economic Simulator.

SALV.: Yes, yes, and difficult though all of this will be, there is always the possibility that we will be able to develop new concepts and theories on the way. 
SAGR.: Your enthusiasm is infectious.

Simp.: Yes, I think he's a bit feverish.

SAGr.: But you are as cool as ever, I see. The sparks of reason appear to have had no effect on your fireproof intellect.

Simp.: Well, that's a little uncalled for. I'm just more cautious, that is all.

SALV.: Which is commendable my dear Simplicio, and it is why in spite of all your faults we love you as a friend, and why I shall trust your recommendation for dinner tonight. See the sun has already set, the dew is falling, the cool of the night is coming on, and a day of thought has given me quite an appetite.

Simp.: Now that is real wisdom, there's a little place down near the river...

SAGR.: Ha, so basic.

SAlv.: As are we all, Sagredo, though some of us are 'looking at the stars',

Simp.: Yes, yes, aren't they beautiful, almost good enough to eat.

SAGR.: Heavens, he's at it again.

Salv.: No, no, I do believe it's a promising sign; wonder is the beginning of curiosity, and in such inquisitive thoughts lie the roots of science.

Simp.: Well, that's awfully kind of you; perhaps as we walk you will explain the ideas to me again.

SALV.: Of course, Simplicio, my patience is infinite. Lead on and I shall start at the beginning.

Exeunt Omnes

Give me a fruitful error any time, full of seeds, bursting with its own corrections. You can keep your sterile truth for yourself. - Vilfredo Pareto commenting on Johannes Kepler 


\section{List of Figures}

2.1 Probability distribution of human height (High school senior male students in Japan). . . . . . . . . . . . . . . . 17

2.2 PDF of companies' declared income. . . . . . . . . . . . . 18

2.3 Double-logarithmic plot of PDF of companies' declared income. 19

2.4 Double-logarithmic plot of CDF for companies' declared income. 21

2.5 Distribution of personal income-tax and income. . . . . . . . . 25

2.6 Evolution of Pareto index of personal income. . . . . . . . . . . . 26

2.7 CDF of personal income in 2000 (Japan). . . . . . . . . . . . 27

2.8 Correlation between the Pareto index, land prices, and stock prices (TOPIX). . . . . . . . . . . . . 28

2.9 CDF of company size: (a) Sales, (b) Income in 2002, Japan. . . 30

$2.10 \mathrm{CDF}$ of sales in various business sectors in 2002 (Japan). . . . . 31

2.11 The CDF of company size in 2001 for: (a) Total capital in France, (b) Sales in France, (c) Number of employees in U.K.. . 32

2.12 Evolution of the Pareto index: (a) France, (b) Italy (c) Spain, (d) U.K., for total capital, sales, and number of employees from 1993 to 2001. . . . . . . . . . . . . . . . . . 33

2.13 gives the $\mathrm{CDF}$ for declared company income for all the data (filled circles) and for all listed companies (open circles). . . . . 34

2.14 PDF of companies' declared income for all the data (filled circles) and data for listed companies (open circles). . . . . . . . 34

2.15 PDF of various Pareto distributions. . . . . . . . . . . . 36

2.16 PDF of various Pareto distributions. . . . . . . . . . . . . . . . 37

2.17 An example of a Lorenz curve. . . . . . . . . . . . . . . . . . . . 39

2.18 The $\mu$-dependence of Gini's coefficient. . . . . . . . . . . . . . 39

2.19 Lorenz curves for various values of $\mu$. . . . . . . . . . . . . . 40

2.20 Definition of the Robin Hood index. . . . . . . . . . . . . . . . 41

$2.21 \mu$-dependence of the Robin Hood index. . . . . . . . . . . . . 41

2.22 The inverse-function method to generate random numbers that obey an arbitrary distribution. . . . . . . . . . . 42

2.23 How to make a staircase plot. Each dot corresponds to a company. 43 2.24 Examples of the staircase plot. . . . . . . . . . . . . . . . 44

2.25 Devil's Staircases for $\mu=0.8,1.0,1.2 \ldots \ldots \ldots$. . . . . . . . . 45

2.26 Share of the largest company. . . . . . . . . . . . . . 47 
2.27 The average share of the second-largest company. . . . . . . . . 49

2.28 Total shares of the top companies. . . . . . . . . . . . . . 49

2.29 Distribution of the share of the top company. . . . . . . . . . . 51

2.30 The value of $\mu$ below which the top $n$-companies achieve a total share greater than $80 \% \ldots \ldots \ldots . \ldots \ldots 2$

2.31 The minimum number, $n$, of the top companies, whose total share is greater than $80 \% \ldots \ldots \ldots 53$

2.32 Various Pareto distributions and the $20 \%$ line. . . . . . . . . . 53

2.33 Dependence of the share of the top $20 \%$ companies on $\mu$. . . . 54

2.34 The range of $m$ where the $80-20$ rule holds. . . . . . . . . . . . 55

2.35 How to obtain the fractal dimension of the size distribution of companies. . . . . . . . . . . . 57

2.36 Fractal dimension of the Devil's Staircase. . . . . . . . . . 58

2.37 Two forces that besiege $\mu=1$. . . . . . . . . . . 63

3.1 Time-series of annual company-sizes for the eight largest electronics and electric-machinery companies (1988 to 2004). . . . . 67

3.2 Time-series of growth-rates for the eight major electronics companies (corresponding to Figure 3.1; 1989 to 2004). . . . . . . 68

3.3 Probability distribution for logarithmic growth-rates of companyincome (2001 to 2002 ; roughly 57,000 companies). . . . . . . . 69

3.4 Probability distribution for logarithmic growth-rates conditioned by company income size (corresponding to Figure 3.3). . . . . . 70

3.5 Probability distribution for growth-rates ((a) sales and (b) profits for the years 2002/2001) . . . . . . . . . . . 71

3.6 Probability distribution for growth-rates ((a) total-assets (companies in France), (b) sales (France), and (c) number of employees per company (UK) for the years 2001/2000.) . . . . . . . 73

3.7 Scatter-plot for company-sizes at successive points in time ((a) sales and (b) profits for the years 2001/2000) . . . . . . . . 77

3.8 Scatter-plot for company-sizes at successive points in time ((a) totalassets (France), (b) sales (France), and (c) number of employees (UK) for the years 2001/2000.) . . . . . . . . . . . . 78

3.9 Three typical examples of copula. . . . . . . . . . . . . . 83

3.10 Copula for company-incomes in the years, 2001 and 2002. . . . 84

3.11 Copula for personal-incomes in the years, 1997 and 1998. . . . 85

3.12 Copula for company-incomes in the year 2001 and its growthrates. . . . . . . . . . . . . 86

3.13 Scatter-plot for personal-incomes (measured by the amount of taxes paid) for two consecutive years (1997 and 1998). . . . . 87

3.14 Probability distribution for growth-rate of personal-income (1997 to 1998). . . . . . . . . . . . . . . . . 88

3.15 Probability distribution for growth-rate of personal-income, 1991 to 1992, corresponding to the bubble collapse in Japan. . . . . 89

3.16 Cumulative distribution for company-size measured by number of employees (whole range; year 2001). . . . . . . . . . . . 92 
3.17 Probability distribution for growth-rates of small and medium companies: ((a) total-assets, (b) debt and (c) sales; years 2001/2000). 94

3.18 Relation between company-size and variance of growth-rate for small and medium companies: ((a) total-assets, (b) debt and (c) sales; years 2001/2000). . . . . . . . . . . . . . . 95

3.19 Annual number of bankruptcies in Japan and total sum of resulting debts (1985 to 2005; calendar-years) . . . . . . . . . . 98

3.20 Annual sum of debts when bankrupted, and ratio to nominal GDP (1996 to 2004; fiscal years). . . . . . . . . . . . . 98

3.21 Cumulative distribution for debt when bankrupted (approximately 16,000 companies bankrupted with debts larger than 10 million yen in 1997). . . . . . . . . . . . . . 99

3.22 Cumulative distribution for life-time before bankruptcy (approximately 16,000 companies bankrupted with debts larger than 10 million yen in 1997). . . . . . . . . . . . . . 100

3.23 Distribution of company's $x$ and two values of $x$ at which profit is maximised under different constraints. . . . . . . . . 105

3.24 Mountain-view with a ridge. . . . . . . . . . . . . . 105

3.25 A mountain-climber and his or her directions. . . . . . . . . . 106

3.26 Landscape for the profit function $\Pi$. . . . . . . . . . . . . . 107

3.27 Contour lines, steepest ascent lines and a ridge for the profit landscape of Figure 3.26. . . . . . . . . . . . 107

3.28 Distribution of company's $x$ (Figure 3.23 ) and the solution of $x$ corresponding to the ridge. . . . . . . . . 108

4.1 Watts-Strogatz $\beta$ model . . . . . . . . . . . . . . . . 112

4.2 A complete graph in which every node is connected to every other within "two degrees of separation" . . . . . . . . . . 119

4.3 Correlation $r$ between degrees of nodes at distance $1 \ldots 122$

4.4 Correlation $r_{4}$ between degrees of nodes at distance 4 . . . . . 122

4.5 Incoming and outgoing links of a listed company in a shareholding or a transaction network . . . . . . . . . 126

4.6 Shareholding network in automobile sector . . . . . . . . . . 126

4.7 The corporate board and directors' network and its reduced graphs . . . . . . . . . . . . . . . . . . . . . 128

4.8 The corporate board network in automobile industry . . . . . . 128

4.9 Transaction network of the automobile industry . . . . . . . . 130

4.10 Patent network and its reduced graphs . . . . . . . . . . . 131

4.11 Network of joint application of patent in automobile industry . 133

4.12 Network of joint patent applications between automobile and electric machinery industries . . . . . . . . . . . . . . 134

4.13 Weighted network of automobile industry . . . . . . . . . . 135

4.14 Outgoing degree distribution of the shareholding network . . . 144

4.15 Degree distribution of the network of joint patent application . 144

4.16 Degree correlation of the shareholding network . . . . . . . . 145 
4.17 Correlation between the degree and clustering coefficients in the shareholding network . . . . . . . . . . . . . 146

4.18 Distribution of total assets, and correlation between degree and total assets . . . . . . . . . . . . . 147

4.19 Distribution of company age, and correlation between outgoing degree and the age . . . . . . . . . . . . . . . 148

4.20 Shareholding network with shareholders belonging to the trans-

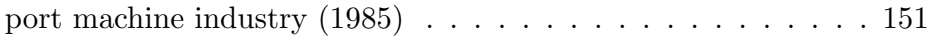

4.21 Shareholding network with shareholders belonging to the transport machine industry (1995) . . . . . . . . . . 151

4.22 Shareholding network with shareholders belonging to the transport machine industry (2000) . . . . . . . . . . . 152

4.23 Shareholding network with shareholders belonging to the transport machine industry (2003) . . . . . . . . . . . . 152

4.24 Shareholding network with shareholders belonging to the electric machinery industry $(1985) \ldots \ldots$. . . . . . . . 153

4.25 Shareholding network with shareholders belonging to the elec-

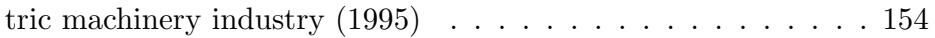

4.26 Shareholding network with shareholders belonging to the elec-

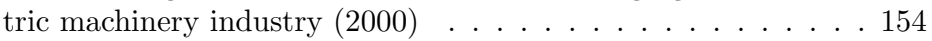

4.27 Shareholding network with shareholders belonging to the electric machinery industry $(2003) \ldots \ldots . \ldots 155$

4.28 Change of degree distribution . . . . . . . . . . . . . . . . . 156

4.29 Change of the long-term shareholding rate and the cross-shareholding rate, and correlation with the power-law exponent . . . . . 157

4.30 Change of degree distribution in a shareholding network . . . . 158

4.31 Distribution of growth rate of sales $X$ and that of costs $Y$. . 160

4.32 Correlation coefficient between growth rates for sales and costs 161

4.33 Cumulative probability distributions of incoming degree and outgoing degree . . . . . . . . . . . . . . . 162

4.34 Standard deviation of residual error and confidence level . . . . 164

4.35 Distribution of correlation coefficient for sales . . . . . . . . . 165

4.36 Network effect on correlation coefficient . . . . . . . . . . 165

4.37 Distribution of correlation coefficient for sales in the overlapping network . . . . . . . . . . . . 166

5.1 behaviour of companies in Gibrat's process . . . . . . . . . 171

5.2 Distribution of company sizes in Gibrat's process . . . . . . . . 171

5.3 Simulated results for sizes and ages of companies . . . . . . 173

5.4 Conversion of the simulated results to the degree distribution . 174

5.5 Conceptual figure of agent-based model: companies interacting through a single bank . . . . . . . . . . . . . . . 177

5.6 balance sheets for companies and a bank . . . . . . . . . . 177

5.7 Value added versus fixed assets for listed Japanese companies

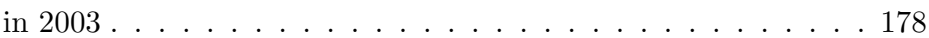


5.8 Emergence of finite probability of bankruptcy with increase of expected profit . . . . . . . . . . . . . . . . 179

5.9 Determination of the interest rate for a company . . . . . . . . 180

5.10 Representative company (left panel) and bank (right panel) . . 182

5.11 Temporal evolution of the agent-based simulation . . . . . . . . 183

5.12 Competition among companies . . . . . . . . . . . . . . . 184

5.13 Temporal evolution of the bank agent . . . . . . . . . . 185

5.14 Size distribution for companies existing eternally . . . . . . . 185

5.15 Size distribution for companies susceptible to bankruptcy . . . 186

5.16 Macroscopic shocks originating from synchronised bankruptcy . 187

5.17 Wealth distribution in a regular network . . . . . . . . . . . 189

5.18 Wealth distribution on a small-world network . . . . . . . . . 190

5.19 Wealth distribution in a random network . . . . . . . . . . . 191

5.20 Time evolution of distribution of wealth across agents on the regular network, corresponding to Figure 5.17. . . . . . . . . 191

5.21 Time evolution of distribution of wealth across agents on the small-world network corresponding to Figure 5.18. . . . . . . . 192

5.22 Time evolution of distribution of wealth across agents on the random network, corresponding to Figure 5.19. . . . . . . . 193

5.23 Interacting company agents . . . . . . . . . . . . . . . . 195

5.24 Multiple Nash equilibrium solutions . . . . . . . . . . . . 198

5.25 Extensive form game . . . . . . . . . . . . . . . . . . . . . . . . . . . . . . . . . . . . . . . .

5.26 Gene . . . . . . . . . . . . . . . . . . . . 201

5.27 Crossover process . . . . . . . . . . . . . . . . . 201

5.28 Mutation process . . . . . . . . . . . . . . . . . . . . . . . . . . . . . . . . . . . . . .

5.29 Payoff and fitness . . . . . . . . . . . . . . . . . 202

5.30 Selection by roulette method . . . . . . . . . . . . 203

6.1 Distribution of $U-D$ and risk capital $E_{p} \ldots \ldots 210$

6.2 Portion of the transaction network in Japan . . . . . . . . . . . 215

6.3 GDP scenario . . . . . . . . . . . . . . . . . 216

6.4 Cumulative probability distributions of sales revenues . . . . 216

6.5 GDP scenarios and cumulative probability distributions of sales revenues . . . . . . . . . . . . . . . . . 217

6.6 Rank-size plots of indebtedness at bankruptcy, comparing origins of bankruptcy . . . . . . . . . . . . . . 222

6.7 Rank-size plots of indebtedness at bankruptcy plotted for comparison between two major causes of bankruptcy (Annual reports on business bankruptcy, published by SMRJ) . . . . . . . 223

6.8 (a) Fraction of bankruptcies due to link effects, (b) Dependence of bankruptcies due to link effects on the amount of indebtedness 224

6.9 Cumulative distributions of (a) in-degree (vendors) and (b) out-degree (customers). . . . . . . . . . . . 227

6.10 A part of the transaction network consisting of a bankrupt company, creditors, and creditors of creditors . . . . . . . . 229

6.11 Model of chain bankruptcy in a transaction network . . . . . . 230 
6.12 Results of the chain bankruptcy simulation . . . . . . . . . 231

6.13 Coarse-grained transaction network . . . . . . . . . . . . 235

6.14 Parameters of the production function for companies belonging

to a Japanese industrial group . . . . . . . . . . . . . . . . 241 


\section{List of Tables}

2.1 Average shares (\%) of the top ten, fifty, and one hundred companies for values of $\mu$ closer to $1 . \ldots \ldots . . \ldots 50$

2.2 The value of $\mu$ below which the top $20 \%$ of companies have a combined share of $75 \%, 80 \%$, and $85 \% \ldots \ldots \ldots 5$

3.1 Parameters of $\tau$ and $\rho$ for copulas . . . . . . . . . . . . 85

4.1 Network indices for 13 automobile companies . . . . . . . . . 136

4.2 Clique of 13 companies belonging to the automobile industry . 140

4.3 Network indices for the whole network, electric machinery, and automobile . . . . . . . . . . . . . . 141

4.4 Network indices of the pharmaceuticals and steel industries . . 142

4.5 Change of shareholding network . . . . . . . . . . . 155

5.1 Strategic form game $1 \ldots \ldots \ldots$. . . . . . . . . . . . . . . . . . . . 196

5.2 Strategic form game $2 \ldots \ldots$. . . . . . . . . . . . . . . 196

5.3 Strategic form game $3 \ldots \ldots$. . . . . . . . . . . 197

6.1 Correspondence between symbols used in corporate finance theory and variables in this book . . . . . . . . . . . 207

6.2 Number of creditors and amount of indebtedness for a bankrupt company . . . . . . . . . . . . . . . . . . 225

6.3 Cases of M\&A in Japanese electronic component industry . . . 233 


\section{Bibliography}

Aitchison, J. and J. A. C. Brown, The Lognormal Distribution, Cambridge University Press, 1957.

Albert, R., H. Jeong, and A. L. Barabási, "Internet: Diameter of the world-wide web," Nature, 1999, 401, 130-131.

Alligood, K. T., T. Sauer, and J. A. Yorke, Chaos: An Introduction to Dynamical Systems, Springer-Verlag, New York, 1997.

Amaral, L. A. N., A. Scala, M. Barthelemy, and H. E. Stanley, "Classes of small-world networks," Proceedings of the National Academy of Sciences, 2000, 97 (21), 11149.

-, S. V. Buldyrev, S. Havlin, H. Leschhorn, P. Maass, M. A. Salinger, H. E. Stanley, and M. H. R. Stanley, "Scaling behavior in economics: I. Empirical results for company growth," Journal de Physique I France, 1997, 7 (4), 621-633.

- , - , ·, M. A. Salinger, and H. E. Stanley, "Power law scaling for a system of interacting units with complex internal structure," Physical Review Letters, 1998, 80 (7), 1385-1388.

Anderson, C., The Long Tail: Why the Future of Business Is Selling Less of More, Hyperion Books, 2006.

Aoki, M., Modeling Aggregate Behavior and Fluctuations in Economics: Stochastic Views of Interacting Agents, Cambridge University Press, New York, 2002.

and H. Yoshikawa, Reconstructing Macroeconomics: A Perspective from Statistical Physics and Combinatorial Stochastic Processes, Cambridge University Press, New York, 2007.

Aoyama, H. and H. Kikuchi, "A new valley method for instanton deformation," Nuclear Physics B, 1992, 369, 219-234.

-, - I. Okouchi, M. Sato, and S. Wada, "Valley views: instantons, large order behaviors, and supersymmetry," Nuclear Physics B, 1999, 553, $644-710$. 
-, H. Yoshikawa, H. Iyetomi, and Y. Fujiwara, "Productivity dispersion: Facts, theory, and implications," Arxiv preprint arXiv:0805.2792, RIETI discussion paper 08-E, 2008, 35.

-, Y. Fujiwara, and W. Souma, "Kinematics and dynamics of ParetoZipf's law and Gibrat's law," Physica A, 2004, 344, 117-121.

- , - , H. Iyetomi, and A.-H. Sato (eds.), Econophysics - Physical approach to Social and Economic Phenomena (Progress of Theoretical Physics, Supplement No.179), Yukawa Institute for Theoretical Physics and The Physical Society of Japan, 2009.

-, Y. Nagahara, M. P. Okazaki, W. Souma, H. Takayasu, and M. Takayasu, "Pareto's law for income of individuals and debt of bankrupt companies," Fractals, 2000, 8, 293-300.

Arrow, K. J., H. B. Chenery, B. S. Minhas, and R. M. Solow, "Capital-labor substitution and economic efficiency," The Review of Economics and Statistics, 1961, 43 (3), 225-250.

Auerbach, F., "Das Gesetz der Bevölkerungskonzentration," Petermanns Geographische Mitteilungen, 1913, 59, 74-76.

Axtell, R.L., "Zipf distribution of US firm sizes," Science, 2001, 293 (5536), 1818-1820.

Barabási, A. L., Linked: How Everything Is Connected to Everything Else and What It Means for Business, Science, and Everyday Life, reissue ed., Plume, 42003.

- and R. Albert, "Emergence of scaling in complex networks," Science, 1999, 286, 509-512.

and Z. N. Oltvai, "Network biology: understanding the cell's functional organization," Nature Reviews Genetics, 2004, 5 (2), 101-113.

Barrat, A., M. Barthelemy, R. Pastor-Satorras, and A. Vespignani, "The architecture of complex weighted networks," Proceedings of the National Academy of Sciences, 2004, 101 (11), 3747-3752.

Berger, S., How We Compete: What Companies Around the World Are Doing to Make it in Today's Global Economy, first ed., Broadway Business, 2005 .

Bergeron, B., Essentials of XBRL: Financial Reporting in the 21st Century, essentials series ed., Johm Wiley \& Sons, Inc., 2003.

Black, F. and M. Scholes, "The pricing of options and corporate liabilities," Journal of political economy, 1973, 81 (3), 637.

Bollobás, B., Random Graphs, Academic Press, 1985. 
Bottazzi, G. and A. Secchi, "Why are distributions of firm growth rates tent-shaped?," Economics Letters, 2003, 80 (3), 415-420.

Bouchaud, J. P. and M. Mezard, "Wealth condensation in a simple model of economy," Physica A: Statistical Mechanics and its Applications, 2000, $282(3-4), 536-545$.

Bouchaud, J.P. and M. Potters, Theory of Financial Risk and Derivative Pricing: from Statistical Physics to Risk Management, Cambridge Univ Pr, 2003.

Brealey, R., S. Myers, and F. Allen, Principles of Corporate Finance, McGraw-Hill/Irwin, 2008.

Brin, S. and L. Page, "The anatomy of a large-scale hypertextual Web search engine," Computer networks and ISDN systems, 1998, 30 (1-7), 107 117 .

Buchanan, M., Nexus: Small Worlds and the Groundbreaking Science of Networks, reprint ed., W W Norton \& Co Inc, 62003.

Caldarelli, G., Scale-free Networks: Complex Webs in Nature and Technology, Oxford University Press, 2007.

Callen, H. B., Thermodynamics and an Introduction to Thermostatistics, second ed., John Wiley \& Sons, 1985.

Champernowne, D. G., The Distribution of Income between Persons, Cambridge University Press, 1973.

Chesbrough, H. W., Open Innovation, first ed., Harvard Business School Press, 2006.

Christensen, L. R., D. W. Jorgenson, and L. J. Lau, "Transcendental logarithmic production frontiers," The Review of Economics and Statistics, 1973, 55 (1), 28-45.

Cobb, C. W. and P. H. Douglas, "A theory of production," The American Economic Review, 1928, 18 (1), 139-165.

Davis, M. D., Game Theory, second ed., Basic Books, New York, 1983.

Delli Gatti, D., E. Gaffeo, M. Gallegati, G. Giulioni, and A. Palestrini, Emergent Macroeconomics: An Agent-based Approach to Business Fluctuations, Springer, 2008.

- , M. Gallegati, and A. Palestrini, "Agents' Heterogeneity, Aggregation, and Economic Fluctuations," in Delli Gatti, D. and Gallegati, M. and Kirman, A., ed., Interaction and Market Structure: Essays on Heterogeneity in Economics, number 484. In 'Lecture Notes in Economics and Mathematical Systems.', Springer-Verlag, 2000, pp. 133-149. 
Dertouzos, M. L., R. K. Lester, and R. M. Solow, Made in America: Regaining the Productive Edge, first ed., MIT Press, 1989.

Erdős, P. and Rényi, A., "On the evolution of random graphs," Publ. Math. Inst. Hung. Acad. Sci, 1960, 5, 17-61.

Falconer, K. J., Fractal Geometry: Mathematical Foundations and Applications, John Wiley \& Sons, 2003.

Farmer, J. D., M. Shubik, and E. Smith, "Is economics the next physical science?," Physics Today, 2005, 58 (September), 37-42.

Freeman, L.C., The Development of Social Network Analysis: A Study in the Sociology of Science, Empirical Press, 2004.

Fu, D., F. Pammolli, S. V. Buldyrev, M. Riccaboni, K. Matia, K. Yamasaki, and H. E. Stanley, "The growth of business firms: Theoretical framework and empirical evidence," Proceedings of the National Academy of Sciences, 2005, 102 (52), 18801-18806.

Fujiwara, Y., "Zipf law in firms bankruptcy," Physica A, 2004, 337, 219230 .

-, "Chain of firms bankruptcy: a macroscopic study of link effect in a production network," Advances in Complex Systems, 2008, 11 (5), 703-717.

- and H. Aoyama, "Large-scale structure of a nation-wide production network," Arxiv preprint arXiv:0806.4280, KUNS-2178, 2008.

-, C. Di Guilmi, H. Aoyama, M. Gallegati, and W. Souma, "Do Pareto-Zipf and Gibrat laws hold true? An analysis with European firms," Physica A, 2004, 335, 197-216.

-, H. Aoyama, and W. Souma, "Growth and fluctuations for smallbusiness firms," in H. Takayasu, ed., Practical Fruits of Econophysics: Proceedings of the third Nikkei Econophysics Symposium, Springer-Verlag, Tokyo 2006, pp. 291-295.

- , - , and - "Growth of firms and networks," in B. K. Chakrabarti, A. Chakraborti, and A. Chatterjee, eds., Econophysics and Sociophysics: Trends and Perspectives, Wiley-VCH Verlag GmbH \& Co., 2006, chapter 4, pp. $99-129$.

-, W. Souma, H. Aoyama, T. Kaizoji, and M. Aoki, "Growth and fluctuations of personal income," Physica A, 2003, 321, 598-604.

Gabaix, X., "The Granular Origins of Aggregate Fluctuations," 2008. SSRN eLibrary, Working Paper Series, http://ssrn.com/paper=1111765.

Galilei, Galileo, Dialogue Concerning the Two Chief World Systems 1632. 
Gallegati, M., G. Giulioni, and N. Kichiji, "Complex Dynamics and Financial Fragility in an Agent Based Model," in Kumar, V. et al., ed., Computational Science and Its Applications - ICCSA 2003, number 2667. In 'Lecture Notes in Computer Science.' Springer-Verlag 2003, pp. 770-779.

Garlaschelli, D., S. Battiston, M. Castri, V.D.P. Servedio, and G. Caldarelli, "The scale-free topology of market investments," Physica A: Statistical Mechanics and its Applications, 2005, 350 (2-4), 491-499.

Gell-Mann, M., The Quark and the Jaguar: Adventures in the Simple and the Complex, Owl Books, 1995.

Gibbons, R., Game Theory for Applied Economists, first ed., Princeton University Press, 1992.

Gibrat, R., Les inégalités Économiques, Librairie du Recueil Sirey, Paris, 1931.

Goldberg, D. E., Genetic Algorithms in Search, Optimization and Machine Learning, Addison Wesley, 1989.

Holland, J.H., Hidden Order: How Adaptation Builds Complexity, Basic Books, 1996

-, Emergence: From Chaos to Order, Oxford University Press, 1998.

Hoover, E. M., "The measurement of industrial localization," Review of Economics and Statistics, 1936, 18 (162-171).

Hull, J. C., Options, Futures, and Other Derivatives, seventh ed., Prentice Hall, 2008.

Ijiri, Y. and H. A. Simon, Skew Distributions and the Sizes of Business Firms, North-Holland, 1977.

Ikeda, Y., H. Aoyama, H. Iyetomi, Y. Fujiwara, and W. Souma, "Correlated Performance of Firms in a Transaction Network," Journal of Economic Interaction and Coordination, 2008, 3, 73-80.

$\cdot, \cdot, \cdot, \cdot, \cdot$, and T. Kaizoji, "Response of firm agent network to exogenous shock," Physica A, 2007, 382, 138-148.

- , W. Souma, H. Aoyama, H. Iyetomi, Y. Fujiwara, and T. Kaizoji, "Quantitative agent-based firm dynamics simulation with parameters estimated by financial and transaction data analysis," Physica A, 2007, 375, 651-667.

Iyetomi, H., H. Aoyama, Y. Fujiwara, Y. Ikeda, and W. Souma, "Agent-based model approach to complex phenomena in real economy," Prog. Theor. Phys. Suppl., 2009, 179, 123-133. 
$\cdot, \cdot, \cdot, \cdot$, and - , "Production copula," 2009. http://arxiv.org/abs/ 0902.1576.

Kesten, H., "Random difference equations and renewal theory for products of random matrices," Acta Mathematica, 1973, 131 (1), 207-248.

Kleiber, C. and S. Kotz, Statistical Size distributions in Economics and Actuarial Sciences, John Wiley \& Sons, 2003.

Kleinberg, J.M., "Authoritative sources in a hyperlinked environment," Journal of the ACM, 1999, 46 (5), 604-632.

Leontief, W. W., The structure of American Economy, 1919-1929: An Empirical Application of Equilibrium Analysis, Harvard University Press, 1941.

Luenberger, D. G., Investment Science, illustrated ed., Oxford Univ Press, 1997.

Mandelbrot, B. B., Fractals and Scaling in Finance: Discontinuity, Concentration, Risk, Springer, 1997.

Manrubia, S. C. and D. H. Zanette, "Stochastic multiplicative processes with reset events," Physical Review E, 1999, 59 (5), 4945-4948.

Mantegna, R.N. and H.E. Stanley, An Introduction to Econophysics: Correlations and Complexity in Finance, Cambridge Univ Pr, 2000.

Milgram, S., "The small world problem," Psychology today, 1967, 2 (1), 60-67.

Milo, R., S. Shen-Orr, S. Itzkovitz, N. Kashtan, D. Chklovskii, and U. Alon, "Network motifs: simple building blocks of complex networks," Science, 2002, 298 (5594), 824-827.

Modigliani, F. and M.H. Miller, "The cost of capital, corporation finance and the theory of investment," The American Economic Review, 1958, pp. 261-297.

Nelsen, R. B., An Introduction to Copulas, second ed., Springer, 2006.

Newman, M. E. J., "Ego-centered networks and the ripple effect," Social Networks, 2003, 25 (1), 83-95.

-, "Random Graphs as Models of Networks," in S. Bornholdt and H. G. Schuster, eds., Handbook of Graphs and Networks, Wiley-VCH Weinheim, 2003, chapter 2, pp. 35-68.

-, "The Structure and Function of Complex Networks," SIAM Review, 2003, 45 (2), 167-256. 
Nirei, M. and W. Souma, "Income distribution and stochastic multiplicative process with reset events," in M. Gallegati, A. P. Kirman, and M. Marsili, eds., The Complex Dynamics of Economic Interaction: Essays in Economics and Econophysics, Springer-Verlag, New York, 2006, pp. 161-168.

Okuyama, K., M. Takayasu, and H. Takayasu, "Zipf's law in income distribution of companies," Physica A, 1999, 269 (1), 125-131.

Ōno, T., Toyota production system: beyond large-scale production, Productivity $\operatorname{Pr}, 1988$.

Owen, G., Game Theory, third ed., Academic Press, 1995.

Pareto, V., Cours D'économie Politique, F. Rouge, Lausanne, 1896.

Pastor-Satorras, R., A. Vazquez, and A. Vespignani, "Dynamical and correlation properties of the Internet," Physical Review Letters, 2001, 87 (25), 258701-258701.

Piore, M. J. and C. F. Sabel, The Second Industrial Divide: Possibilities for Prosperity, first ed., Basic Books, New York, 1984.

Pratt, J., Financial Accounting in an Economic Context, seventh ed., John Wiley \& Sons, 2008.

Press, W. H., S. A. Teukolsky, W. T. Vetterling, and B. P. Flannery, Numerical Recipes in C: The Art of Scientific Computing, second ed., University of Cambridge, 1992.

Ravasz, E., A. L. Somera, D. A. Mongru, Z. N. Oltvai, and A. L. Barabasi, "Hierarchical Organization of Modularity in Metabolic Networks," Science, 2002, 297 (5586), 1551.

Redner, S., "Random multiplicative processes: an elementary tutorial," American Journal of Physics, 1990, 58, 267-273.

Sapolsky, R., "Sick of poverty," Scientific American, 2005, 293 (6), 92-99.

Saramäki, J., M. Kivelä, J.P. Onnela, K. Kaski, and J. Kértesz, "Generalizations of the clustering coefficient to weighted complex networks," Physical Review E, 2007, 75, 027105.

Shen-Orr, S.S., R. Milo, S. Mangan, and U. Alon, "Network motifs in the transcriptional regulation network of Escherichia coli," Nature Genetics, 2002, 31 (1), 64-68.

Shirata, C. Y., "An attempt to rate compamies based on bankruptcy prediction model," the 16th Asia-Pacific Conference on International Accounting Issues, 2004.

Solomon, S. and M. Levy, "Power laws are logarithmic Boltzmann laws," International Journal of Modern Physics C, 1996, 74, 595-601. 
Sornette, D., "Multiplicative processes and power laws," Physical Review E, 1998, 57 (4), 4811-4813.

-, Critical Phenomena in Natural Sciences: Chaos, Fractals, Selforganization, and Disorder: Concepts and Tools, second ed., Springer-Verlag, Berlin, 2004 .

- and R. Cont, "Convergent multiplicative processes repelled from zero: power laws and truncated power laws," Journal de Physique I France, 1997, $7(3), 431-444$.

Souma, W., Y. Fujiwara, and H. Aoyama, "Small-world effects in wealth distribution," Arxiv preprint cond-mat/0108482, 2001.

- , - , and -, "Wealth Distribution in Scale-Free Networks," in T. Terano, H. Deguchi, and K. Takadama, eds., Meeting the challenge of social problems via agent-based simulation: Post-proceedings of the second International Workshop on Agent-Based Approaches in Economic and Social Complex Systems, Springer-Verlag Inc. (New York), 2003, pp. 37-49.

Stanley, Eugene, "Econophysics and the current economic turmoil," APS news, 2009, 8 December.

Stanley, M. H. R., L. A. N. Amaral, S. V. Buldyrev, S. Havlin, H. Leschhorn, P. Maass, M. A. Salinger, and H. E. Stanley, "Scaling behaviour in the growth of companies," Nature, 1996, 379, 804-806.

Steindl, J., Random Processes and the Growth of Firms: a Study of the Pareto Law, Griffin, London, 1965.

Stiglitz, J. and B. Greenwald, Towards a New Paradigm in Monetary Economics, first ed., Cambridge University Press, 2003.

Sutton, J., "Gibrat's legacy," Journal of Economic Literature, 1997, 35 (1), $40-59$.

Takayasu, H., A.-H. Sato, and M. Takayasu, "Stable infinite variance fluctuations in randomly amplified Langevin systems," Physical Review Letters, 1997, 79 (6), 966-969.

- ed., Empirical Science of Financial Fluctuations: the Advent of Econophysics Springer (Tokyo) 2002.

- , ed., The Application of Econophysics Springer (Tokyo) 2004.

-, ed., Practical Fruits of Econophysics Springer (Tokyo) 2006.

Tsallis, C., "Possible generalization of Boltzmann-Gibbs statistics," Journal of Statistical Physics, 1988, 52 (1), 479-487.

Varian, H. R., Microeconomic Analysis, third ed., W. W. Norton \& Company, New York, 1992. 
- , Intermediate Microeconomics, seventh ed., W. W. Norton \& Company, New York, 2005.

von Neumann, J. and O. Morgenstern, Theory of Games and Economic Behavior, Princeton University Press, 1944.

Waldrop, M., Complexity: The Emerging Science at the Edge of Chaos, Simon and Shuster, New York, 1992.

Watts, D.J., Small Worlds: the Dynamics of Networks between Order and Randomness, Princeton University Press, 1999.

-, Six Degrees: The Science of a Connected Age, W W Norton \& Co Inc, 2 2003.

and S.H. Strogatz, "Collective dynamics of 'small-world' networks.," Nature, 1998, 393 (6684), 440.

Zipf, G. K., Human Behavior and the Principle of Least Effort: An Introduction to Human Ecology, Addison-Wesley, Cambridge MA, 1949. 


\section{Index}

$\underline{A}$

account receivable .............219

adaptability $\ldots \ldots \ldots \ldots \ldots \ldots \ldots$

additive stochastic process .......170

adjacency matrix ............. 189

agent $\ldots \ldots \ldots \ldots \ldots \ldots \ldots \ldots 63,169$

company $-\ldots \ldots \ldots \ldots \ldots 194$

economic - ............. 169

representative $-\ldots \ldots \ldots \ldots 181$

agent-based model..............176

agent-based simulation ......... 169

aging effect................. 175

Amaral, Luls AntÛnio Nunes.... 175

Anderson, Chris ................ 59

annual security report ......... 125

Arrow, Kenneth J..............101

assortativity .................. 117

asymptotic behaviour...........23

Auerbach, Felix...............22

authority ................... 117

autonomy ................... 194

averaged

— degree....

- path length

115,118

.113, 137

B

BA model .............. 175, 193

backward induction.............198

Bacon, Kevin

- game ............. 123

balance sheet......16, 96, 176, 206

Bali ....................... 29

BIS . ..................... 237

bankruptcy ............. 96, 220

— criterion ............... 179

— due to link effect........220

— probability .............166 chain - ...........181, 220

origin for $-\ldots \ldots \ldots \ldots \ldots 220$

synchronised - .......... 186

Barabási, Albert-László..........116

Basel I ..................... 181

$\beta$ model .....................115

bidirectional link .............. 127

bipartite graph ............... 127

Black, Fischer ............... 213

Black-Scholes formulae ........ 213

Boltzmann distribution ..........6 63

borrowing rate .............. 207

Brin, Sergey ................. 117

bubble economy ............. 26

business

— entry .............. 212

— environment ............2238

— exit.................212

— information system ..... 242

— model.................234

— process ............... 238

— synergy ...............232

bypass link ................ 239

c

capital

— adequacy requirements...237

— asset pricing model ..... 207

— deficit ............... 231

- structure................209

cash flow statement............206

CDF ..................... 20

central limit theorem ............ 171

centrality .................. 117

betweenness - ........130, 138

degree - . . . . . . . . . . 135

centre of mass ................ 149 
CES production function ........ 101 chain bankruptcy .......... 181, see bankruptcy, 220

Champernowne, David G. .......91

chaos ........................ 3

Christensen, Laurits R.......... 102

clique ...................... 139

closed innovation .............. 238

cluster.........................114

clustering coefficient ... 113, 137, 146

global $-\ldots \ldots \ldots \ldots \ldots \ldots 114$

weighted - . ............ 137

coarse grained transaction network 236

coarse graining ............... 124

Cobb, Charles W............... 101

company

bankruptcy of - .......96, 220

growth-rate of - .........68

lifetime of $-\ldots \ldots \ldots \ldots \ldots 100$

size of - ...........16, 29

company agent .............. 194

competitive environment........214

complete graph .......... 118, 139

complex system ............ 117

component

out-sourced $-\ldots \ldots \ldots \ldots \ldots 9$

self-manufactured $-\ldots \ldots \ldots 9$

conditional probability density...145

connected component........... 127

consolidation ................ 232

constant elasticity of substitution 101

copula.................... 82

corporate finance........................ 205

corporate valuation...........206

corporate value..............206

correlation

— coefficient..79, 117, 121, 158, 164

$1-$ link $-\ldots \ldots \ldots \ldots \ldots \ldots 164$

2-link - . . . . . . . . . . . 166

degree $-\ldots \ldots \ldots \ldots \ldots 117$

degree-degree —...145, 150, 153

covariance.............. 121, 208

CRD (Credit Risk Database)...v, 92

credit crunch .................226

creditor .................. 220
Cremonini, Cesare .............. 2

critical phenomena ............. 117

critical point.................662

cross-shareholding . . ..... 127, 156

- rate...............156

cumulative distribution function . . 20

cumulative probability

joint $-\ldots \ldots \ldots \ldots \ldots \ldots, 82$

\section{D}

deal on credit ............... 219

debt payable ............... 207

debtor.......................220

degree...............117, 226

- -degree correlation. . 145, 150, 153

— centrality ......................... 135

— correlation ............ 117

— distribution.... 115-117, 142, 153,226

cube average of $-\ldots \ldots \ldots 121$

incoming — ..... 125, 142, 153

out- - ................. 125

outgoing —...125, 142, 147, 153

degree distribution ............ 174

Delli Gatti, Domenico .......... 176

demand ................... 4

derivative financial instrument...213

Descartes, Rene................ 1

detailed balance......... 76, 79, 80

— and Gibrat's law ......... 80

— and power-law .......... 80

- of personal income....... 88

breakdown of - .......... 89

mathematical express of - . . 79

statistical test of $-\ldots \ldots \ldots 79$

devil's staircase..........44, 57

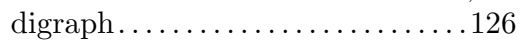

directed graph ....... 126, 153, 218

discount rate ............. 207

distribution ............... 5, 17

— for company's lifetime....100

— for debt when bankrupted.99

— of city size ...........22

— of company income.... 18, 21

— of European companies....33 


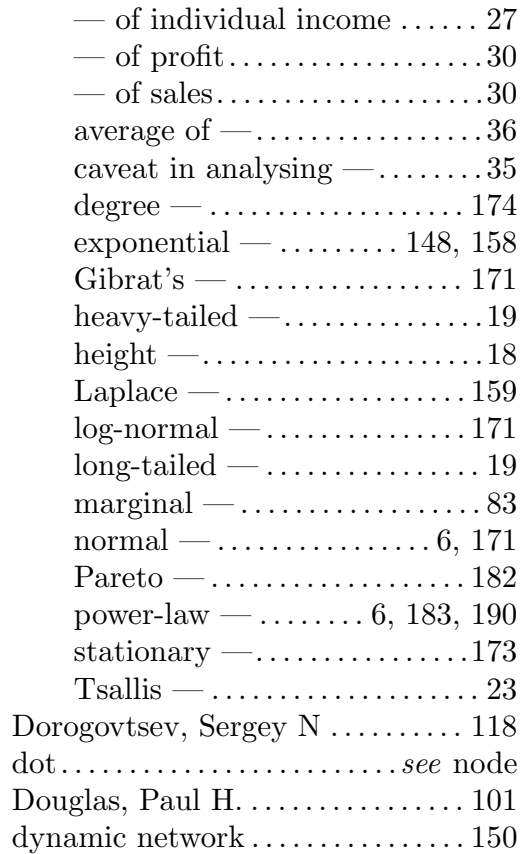

E

economic agent........4, 16, 169

econophysics ...........4, 28

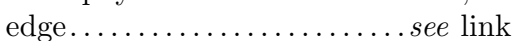

EDI . see electronic data interchange

EDINET . . . . . . . . . . . . . 125

elasticity of substitution ........ 101

electronic commerce .......... . 243

electronic data interchange . . . . . 243

enterprise resource planning . . . . 242

equity .................. 231

Erdős, Paul...................112

ERP see enterprise resource planning

Euler's constant .............46

Euler, Leonhard ............. 112

exponential distribution.....100, 158

eXtensible Business Reporting Language 242

eXtensible Markup Language . . . 242

extensive quantity ............ 101

\section{F}

fat tail ............ 19, 83 financing $\ldots \ldots \ldots \ldots \ldots \ldots 218$

flexible specialisation . . . . . . . . . 238

flow .................. 15

fluctuation .........6 6, 63, 65

spatial - .............69 69

temporal —.............69

four mega banks . . . . . . . . . . . . . 149

fractal............. 55, 117

— dimension...........56

free cash flow ..............206

$\underline{\mathbf{G}}$

Galilei, Galileo ........... xi, 2

Gallegati, Mauro ............ 176

game

- tree .................... 198

non-cooperative - . . . . . . 195

game theory . . . . . . . . . . 194

$\Gamma(z) \ldots \ldots \ldots \ldots \ldots \ldots \ldots \ldots \ldots \ldots \ldots \ldots \ldots \ldots$

$\gamma_{E} \ldots \ldots \ldots \ldots$ see Eler's constant 46

gatekeeper ..............130, 139

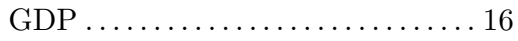

gene.................... 199

general equilibrium theory .......4 4

Gibrat, Robert ............. 74

Gibrat's distribution........... . 171

Gibrat's law ..... 71, 72, 75, 80, 170

— and detailed balance ... . . 80

— for small and medium enterprises................93

— of personal income...... 88

breakdown of - . . . . . . . 89

mathematical expression of -74

Gibrat's process........170, 182, 192

Gini coefficient...........38, 91

going concern .......... 176, 179

golden ratio . . . . . . . . . . . . 57

Google...............660, 118

graph

- reduction . . . . . . . . . 127

— theory .............. 112

bipartite - . . . . . . . . . 127

complete - ......... 139, 164

directed - ......126, 127, 153

multiple - . . . . . . . . . 132

undirected - . . . . . 126, 135 
Graphviz..................... 129

growing network ............. 150

growth-rate.................68

— dependence on size.......93

- distribution (European companies) ..............72

— distribution (SME)........93

- distribution for profits (Japan) 72

- distribution for sales (Japan) 72

- for companies' incomes (Japan) 69

— of personal income.......88

logarithmic - .......... 68

size-dependence of $-\ldots \ldots . .72$

\section{H}

heavy-tail ................... 19

hierarchical

— network.............2239

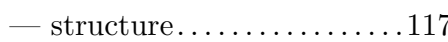

hierarchy ................. 147

high-income individuals ..... .27, 90

horizontal division of work ... .8, 236

hub...........117, 145, 150, 153

I

in-degree .............. 125, 226

income statement ........... 16, 206

industrial group .............. 232

industry reorganisation ......... 232

innovation.................. 124

intensive quantity ............ 101

interaction..................6 63

- between companies. . 159, 163

interest rate ............ 102, 180

interlocking directors' network . . 127

intermediate goods ............ 218

intrinsic value of technology . . . . 238

inverse function method .........44

inverted U-shaped curve hypothesis26

invested capital..............207

investment portfolio ........... 125

J

Japanese bubble.............89 joint probability $\ldots \ldots \ldots \ldots \ldots \ldots 76$

just-in-time production .......... 11

$\mathrm{K}$

Kanban system ................ 11

Kapteyn, Jacobus ............. 74

Keiretsu.....................232

Kendall's $\tau \ldots \ldots \ldots \ldots 84,148,174$

Kendall's rank correlation........ see Kendall's $\tau$

Kesten's process.............. 172

Keynes, John Maynard .......... 91

Kleinberg, Jon................117

Kolkata......................29

Köningsberg bridge problem ..... 112

Kuznets, Simon...............26

$\mathbf{L}$

labour.....................218

land price $\ldots \ldots \ldots \ldots \ldots \ldots \ldots 27$

Laplace distribution ........... 159

largest connected component140, 143 law

Weber-Fechner - ........... 173

law of proportionate effect....... 74

Leontief, Wassily .............. 101

line .................... see link

link ...................... 112

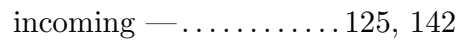

outgoing - ......... 125, 142

load .....................2239

log-normal distribution ...24, 74, 171

long tail............. 19, 48, 59

long-tailed distribution...........see distribution

long-term shareholding rate.... 156

loop ........................ 112

Lorenz curve .................. 38

$M$

M\&A................232, 236

macroeconomic trend ...........214

macroeconomics ............... 4

majour shareholder data.... 125, 150

Mandelbrot, Benoit.............55

marginal distribution .......... 83 


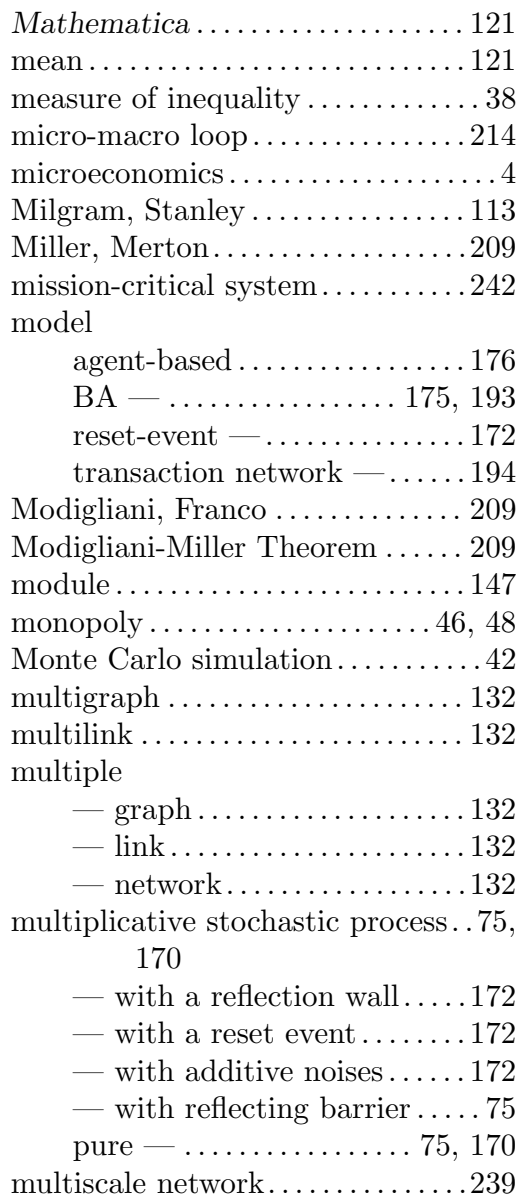

- of joint application of patent 131

— science................ 111

- spectrum ............ 117

corporate board - ......... 127

dynamic - ............. 150

growing - . . . . . . . . . 150

hierarchical - ...........239

innovation - ......... 124, 143

interlocking directors' - . . 124, 127

multiple - ............ 132

multiscale —.............239

overlapping — ............ 163

personnel-sharing - .......63 63

random - .......... 112, 115

regular $-\ldots \ldots \ldots \ldots \ldots \ldots 112$

scale-free - . 116, 120, 143, 157, 175

shareholding - ...63, 124, 125, $142,150,163,172$

small-world - . . . . . . 115, 190

static - ............... 149

transaction -.63, 124, 129, 163, 193

undirected - ............. 132

weighted - .............. 135

network science........... 7, 143

Network Workbench............ 129

no routine task ................. 242

node ..................... 112

— strength..........135, 137

adjacent — ......... 136, 145

non-cooperative game ......... 195

noncorrelation test ............ 161

normal distribution ..... 24, 61, 171

$\underline{0}$

obedience to authority .........113

oligopoly ............46, 61, 155

open innovation ............. 238

open system $\ldots \ldots \ldots \ldots \ldots \ldots \ldots$

order statistics................ 83

out-degree................... 226

overlapping network........... 163 


\section{$\mathbf{P}$}

Page, Lawrence Edward ......... 117

PageRank ................... 117

Pajek ...................... 129

Pareto.......................117

Pareto, Vilfredo............6, 22

Pareto distribution ...... 23, 80, 182

$$
\begin{aligned}
& -(\mathrm{A}) \ldots \ldots \ldots \ldots \ldots \ldots \ldots \ldots \\
& -(\mathrm{B}) \ldots \ldots \ldots \ldots \ldots \ldots \ldots \\
& -(\mathrm{C}) \ldots \ldots \ldots \ldots \ldots \ldots \ldots
\end{aligned}
$$

Pareto exponent ...........23, 155

Pareto principle ............... 50

Pareto's 80-20 rule..............50

Pareto's law ................ 23, 30

path length.................. 136

payoff ...................... 195

PDF ........................17

perfect capital market ........... 209

perfect information ............. 196

personal income ...............23

— and bubble economy ..... 26

detailed balance for $-\ldots \ldots 88$

distribution of - ......... 25

Gibrat's law for $-\ldots \ldots \ldots . .88$

growth-rate of - ...........88

phase.......................62

- transition .............662

oligopolistic - ...........662

pseudo-equal $-\ldots \ldots \ldots \ldots 62$

phase transition ............... 117

player ........................ 195

Poisson

- distribution ............ 115

- random network ........ 115

Pólya, George.................................... 112

positivism ................... 113

power

- exponent..............19

- law ................6 6

power-law .................. 19

— and detailed balance .....8 80

— and Gibrat's law .......... 80

— distribution .... 116, 143, 147

— exponent.......143, 147, 161

- function ................ 145

- region................93 breakdown of $-\ldots \ldots \ldots \ldots 89$

power-law distribution...... 183, 190

preferential attachment......... 175

prisoner's dilemma ............ 197

probability density function...17, 143

probability mass function ........ 17

process

geometric Brownian —..... 173

Gibrat's — . . . . . . . . . . 182, 192

Kesten's - .............. 172

product development ..........238

production function .... 101, 178, 240

transcendental logarithmic -102

profit...................102, 207

- maximisation ........... 102

profit and loss, P/L...........96

profit maximisation.........176, 178

profitability ................. 241

pseudo-equality ................6 62

pseudo-science................52

psychophysics ................ 173

Ptolemaic theory ............... 2

purchasing power parity ......... 29

$\underline{\mathbf{R}}$

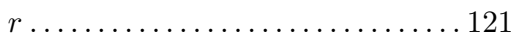

random network .......... 112, 115

random numbers..............43

rank-size plot......20,43, 143, 147

rate

interest - .............. 180

rationality .................... 196

bounded - ............. 196

real option ................... 213

reflection law ............. 80, 81

regular network.................................

relationship.................241

Rényi, Alfréd.................. 115

representative agent .......... 181

residual error ............... 163

return of invested capital ...... 207

return on equity ..............237

ridge theory ............ 102, 104

ripple effect ................. 228

risk. . . . . . . . . . . . . . . . . . . . 159

business - ............ 211 
— -free asset.............208

- asset..................208

— management ...........226

- neutral method ......... 214

- premium.............208

assets of $-\ldots \ldots \ldots \ldots \ldots \ldots 89$

credit - ...... 226, 231, 237

Idiosyncratic - ...........209

market - . . . . . . . . . . . . . . 209

risk capital .............27, 209

risk management..........61, 209

Robin Hood coefficient.............38

robustness.....................2239

ROE...........see return on equity

roulette .................... 203

routine task

.242

\section{$\underline{S}$}

SAF2002 ................... 166

scale-free.................... 117

— network...116, 120, 143, 157, 175

Scholes, Myron .................. 213

science of complex systems......... 3

self-similarity . . . . . . . . . . . 56

shareholding network..125, 142, 150, 163,172

shortest path

.113

silver ratio...................57

Simon, Herbert A. ..............22

Simplicio .................... 2

simulation

agent-based $-\ldots \ldots \ldots \ldots 169$

six degrees of separation .... 113, 118

small and medium-sized companies91

— and SMRJ .............. 99

credit risk database for $-\ldots .92$

distribution of $-\ldots \ldots \ldots . .99$

Gibrat's law for $-\ldots \ldots \ldots . . .93$

growth-rate of $-\ldots \ldots \ldots \ldots 93$

small-world .............113, 118

- network..........115, 190

— problem .............. 115

Smith, Adam................. 178

SMRJ ........................ . 220

social network analysis ........ 112
Spearman's $\rho \ldots \ldots \ldots \ldots \ldots \ldots . \ldots 84$

split-up...................236

staircase plot .............. 43

standard deviation ............ 121

static network ............... 149

stationary distribution........... 173

stationary process ............. 159

statistical mechanics......4, 17, 112

steepest-ascent line ........ 108, 109

stochastic process

additive $-\ldots \ldots \ldots \ldots \ldots 170$

stock ..................... 15

stock holdings data.......... 125

strategic

— form .................. 195

strategy ....................... 195

mixed - . . . . . . . . . . . 198

pure - . . ............. 198

stretched exponential distribution.38

Strogatz, Steven ................. 113

strongly connected component... 236

subgraph ................... 139

sum of individual components ... 241

supply ..................... 4

SCM ..................... 242

supply chain management . . . . . . 242

synchronised bankruptcy ........ 186

synergy......................240

TamadaDB..................143

Tax Commission in Japan ........ 91

tax rate................... 207

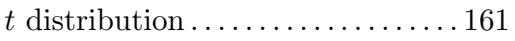

TOPIX .....................27

transaction network.... 163, 193, 217

— model.................194

downstream of - ........218

upstream of - ............218

transition point................6 62

tree ...................... 112

U

universal law ................ 7

V

vagueness ..................... 239 
value added.............178, 218

Varian, Hal ....................60

vertex................... see node - strength ............. 135

vertical integration..........8, 236

W

Walras, Léon ....................4

Watts, Duncan ................. 113

wealth.................... 188

clustering of $-\ldots \ldots \ldots \ldots 191$

Weber-Fechner Law ............ 173

Weibull distribution ............ 38

weighted

— average cost of capital . . 207

— clustering coefficient ..... 137

— network............... 135

$\underline{x}$

XBRL ....... see eXtensible Business Reporting Language

XML.........see eXtensible Markup Language

$\underline{Z}$

Zipf's law ................. 23 


\section{About the authors}

Aoyama, Hideaki Professor of Physics, Kyoto University (Japan).

Hideaki received his Ph.D. from Caltech in 1982 and studied High Energy Physics at SLAC as a postdoctoral fellow, and Harvard University as a visiting scholar, and Northeastern University (Boston) as an lecturer. He is an Advising Council for Credit Risk Database (Tokyo) and a Special Advisor on Physical Sciences for Renewable Energy Foundation (London).

Fujiwara, Yoshi Research Fellow at Advanced Telecommunication Research Institute International (ATR), and Adjunct Lecturer at Kyoto University (Japan).

Yoshi received his Ph.D. from Tokyo Institute of Technology in 1992 and studied General Relativity and Quantum Cosmology at Yukawa Institute (Kyoto) as a postdoctoral fellow, and Institute of Theoretical Physics, University of California (Santa Barbara) as a visiting researcher. He was also engaged in research in Econophysics at Department of Economics, Universita' Politecnica delle Marche (Italy) with Mauro Gallegati.

Ikeda, Yuichi Senior Researcher at Hitachi Research Laboratory, Hitachi Ltd.

Yuichi received his Ph.D. from Kyushu University in 1989 and studied Experimental High Energy Physics at Institute of Nuclear Science at Tokyo University as a postdoctoral fellow, Brookhaven National Laboratory as a collaborator on the AGS-E-802 project, and University of California, Berkeley as a visiting industrial fellow. He worked as a senior researcher at Hitachi Research Institute during 2005 to 2008 before moving to his current position.

Iyetomi, Hiroshi Professor of Physics, Niigata University (Japan). Hiroshi received his Ph.D. from the University of Tokyo in 1984 and continued to study strongly-coupled plasma physics as an assistant professor there. He worked at Hitachi Ltd. as a researcher before moving on to the current position. Also he studied condensed matter physics at Argonne National Laboratory as a research associate, at Louisiana State University as a visiting associate professor, and at Delft University of Technology as a visiting fellow.

Souma, Wataru Associate Professor of Physics, College of Science and Technology, Nihon University (Japan), Research Fellow at Advanced Telecommunication Research Institute International (Japan), and Visiting Associate Professor, Institute of Economic Research, Hitotsubashi University (Japan)

Wataru received his Ph.D. from Kanazawa University in 1996 and studied High Energy Physics at Kyoto University. 
Yoshikawa, Hiroshi (Preface) Professor of Economics, University of Tokyo (Japan).

Hiroshi received his Ph.D. from Yale University in 1978. He served as the president of the Japanese Economic Association in 2002 and is currently a member of the Council on Economics and Fiscal Policy in Japan. Among his several books, the latest is "Reconstructing Macroeconomics - A Perspective from Statistical Physics and Combinatorial Stochastic Processes", Cambridge University Press (2007). 